AperTO - Archivio Istituzionale Open Access dell'Università di Torino

\title{
Education and health in Europe
}

\section{This is the author's manuscript}

Original Citation:

Availability:

This version is available http://hdl.handle.net/2318/156560

since 2018-04-24T02:45:31Z

Published version:

DOI: $10.1080 / 00036846.2017 .1361013$

Terms of use:

Open Access

Anyone can freely access the full text of works made available as "Open Access". Works made available under a Creative Commons license can be used according to the terms and conditions of said license. Use of all other works requires consent of the right holder (author or publisher) if not exempted from copyright protection by the applicable law. 


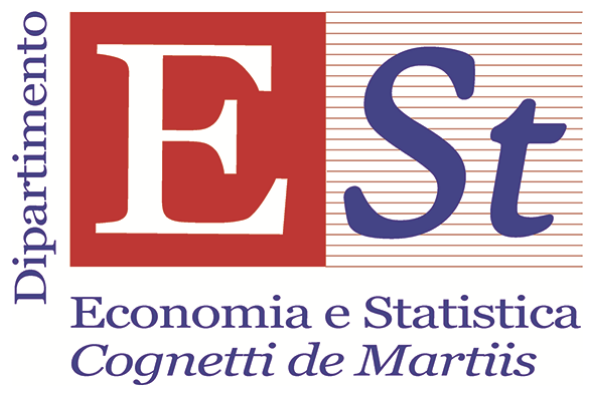

\section{Working Paper Series}

\section{EDUCATION, HEALTH AND SUBJECTIVE WELLBEING IN EUROPE}

\section{LEONARDO BECCHETTI, PIERLUIGI CONZO and FABIO PISANI}





\title{
Education, health and subjective wellbeing in Europe
}

\author{
Leonardo Becchetti*, Pierluigi Conzo ${ }^{\dagger}$, Fabio Pisani ${ }^{\ddagger}$ \\ * Dept. of Economics, University of Rome Tor Vergata, Via Columbia 2, I-00133 Roma; email: \\ becchetti@economia.uniroma2.it \\ †Dept. of Economics and Statistics “Cognetti de Martiis”, University of Turin \& CSEF, Naples \\ ‡ Dept. of Economics, University of Rome Tor Vergata
}

This version: January $2015 \S$

\begin{abstract}
The productive and allocative theories predict that education has positive impact on health: the more educated adopt healthier life styles and use more efficiently health inputs and this explains why they live longer. We find partial support for these theories with an econometric analysis on a large sample of Europeans aged above 50 documenting a significant and positive correlation among education years, life styles, health outputs and functionalities. We however find confirmation for an anomaly already observed in the US, namely the more educated are more likely to contract cancer. Our results are robust when controlling for endogeneity and reverse causality in IV estimates with instrumental variables related to quarter of birth and neighbours' cultural norms.
\end{abstract}

Keywords: health satisfaction, education, life satisfaction, public health costs.

JEL numbers: I21 Analysis of education; I12 Health Behavior; I31 General welfare, wellbeing

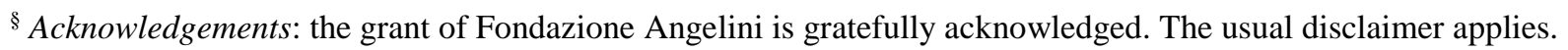




\section{Introduction}

The impact of education on health is a well-known stylized fact in the medical literature. Olshansky, et al. (2012) look at mortality data between 1990 and 2008 in the US and estimate a higher than 10 year difference in life expectancy between white males with less than 12 years and more than 16 years of education. Meara et al. (2008) show that all gains in life expectancy at age 25 in the US between the 1980s and the 1990s occurred among the better educated thereby raising educational differentials in life expectancy by 30 percent. The difference in Europe is narrower but still relevant. Corsini (2010) calculates that in 2007 life expectancy at age 50 for males was 5 years higher for men in Czech Republic, around 3 years higher in Belgium and in Italy, and around 2 years higher in Norway for those with tertiary education vis-à-vis those with primary education. Female gaps are slightly lower but still significant.

The contribution of the economic literature to the interpretation of these stylized facts is in formulating theories, identifying causal links and disentangling the impact of different drivers of the observed findings net of all the other controls with econometric analysis. First of all, we may expect that part of the above mentioned evidence is explained by channels different from that of the direct nexus between education and health (i.e. the more educated may be less likely to incur in car accidents). Second, we may for instance expect that differences in income determined by returns to schooling explain an important part of the health differential between the more and less educated ${ }^{1}$ and we may therefore be interested in distinguishing between direct and indirect (i.e. through income) education effects.

Beyond the above mentioned concurring factors the empirical nexus between education years and health outcomes remains however the main well-established empirical finding accounting for the correlation between education and life expectancy (see, e.g., Grossman, 2006, for a survey). Such

\footnotetext{
${ }^{1}$ Education years affect earned income through returns to schooling and thereby reduce the cost of health allowing
} richer individuals to buy more expensive medical treatments and healthier foods. 
nexus has been interpreted in the sense of a causality link going from education years to health with two main rationales.

The first rationale refers to the "productivity argument" and follows the Becker (1965) theoretical framework where individual utility accrues from the enjoyment of a set of non market goods which include health. Non market goods can be "produced" with a productive function having as inputs time and market goods (such as diet, cigarette smoking, alcohol use and medical care), with some of them having positive while other negative effects on health. In this production function education years act as Hicks neutral technical progress increasing the productivity of inputs (Kenkel et al., 2006). Due to this productivity effect it is possible to demonstrate that the demand of the nonmarket output "health" grows in education years under reasonable assumptions on income elasticities and elasticities of substitution between health and other goods. The intuition behind the model is that education raises the impact of time and market goods on health. This is because educated individuals know how to use in a more productive way the time spent - directly or indirectly through doctor visits - for health advice and to understand their health conditions and the market goods which contribute to it, while lack of education may lead to misuse and ignorance on the effectiveness of some therapies.

The second rationale refers to the "allocative efficiency" argument assuming that education years do not act just as technological progress in the production function of health but also induce individuals to change the input mix (see, among others, Rosenzweig and Schultz, 1983 and Ross and Mirowsky, 1999). The intuition is that the more educated have superior information about the true nature of the production function and therefore make better choices among market inputs of the health production function (i.e. they adopt healthier life styles since they are more informed about the harmful effects of smoking, drinking and following a bad diet).

As is well known this strand of theoretical and empirical research may provide important contributions to the human capital literature: if the two above described causality links exist they reinforce the importance of education years on productivity and returns to schooling given that the 
more educated are more productive not just because of their superior knowledge and skills but also because they are likely to lose less working days due to health problems.

\subsection{Related studies and endogeneity issues}

Empirical findings confirm the positive relationship between education and health postulated by the theoretical literature. Effects of education on health behaviors are reported, among others, by Kenkel (1991) and Meara (2001). Wagstaff (1986), Gilleskie and Harrison (1998) and Erbsland et al. (1995) find support for the productive efficiency model, while results of Kenkel (1991), confirming the effect of education on healthier life styles support the allocative efficiency model. Ross and Wu (1995) find a positive and significant gradient for both the direct relationship and the indirect relationship mediated by life habits. Cutler and Lleras-Muney (2006) find that higher levels of education are linked to higher health status and are positively associated to healthier life styles. Other papers show that the more educated live longer in the US (Christenson and Johnson, 1995; Deaton and Paxson, 2001) and in other countries (Mustard, 1997; Manor et al., 1999; Liang, et al., 2000), while Kunst and Mackenack (1994) confirm the result for Europe. Elo and Preston (1996) document that the education gradient loses some of its importance for older ages on mortality rate. It is reasonable to assume that this phenomenon is due to a kind of survival effect (Lynch, 2003). Among the various effects, education improves capacity of learning when a process of learning is possible (as in chronic diseases) (Goldman and Lakdawalla, 2001 and Case et al., 2005). Ross and Mirowsky (1999) observe that the more educated have a higher sense of control and self-esteem, both of them related to a superior health. Meara (2001) and Currie and Moretti (2003) show that mother's education improves the health of children.

Exploiting the results of quasi-natural experiments (i.e. testing if the introduction of minimum schooling laws in some countries led to a higher health) Arendt (2005), Lleras-Muney (2005), and Oreopolous (2007) show the presence of a specific causal effect of education on health. The relevance of the causal nexus seems reinforced by evidence showing that innovation which reduces 
skills required to use drugs has negative effects on the education-health gradient. Goldman and Lakdawalla (2002) document that the introduction of beta blockers which drastically simplify therapies against hypertension reduce the morbidity gap between more and less educated.

The nexus between education and health may as well conceal a reverse causality nexus or a spurious link where a third omitted driver affecting both variables is the true factor explaining their observed correlation (see Card, 1999 and 2000).

The rationale for the endogeneity problem is that factors such as parental investments into their children, parental or family background, or differences in non-cognitive traits or time preferences (Braakman, 2010) may produce a spurious relationship between education and health. Parents with lower time impatience (or more altruistic preferences toward their children) invest more in their children education. At the same time their lower time impatience leads them to care more for their health and for that of their children. Hence the observed correlation between education years and health may be spurious and partially driven by parental preferences. Still in this case endogeneity is likely to be mixed with direct causality since remote factors, such as parental preferences, affecting education years do not prevent education years from exerting a positive impact on health through the three channels described above. Empirical evidence on the reverse causality nexus is as well documented by the fact that low health levels of children are linked to low levels of education (see Edwards and Grossman, 1979; Chaikind and Corman, 1991; Currie, 2000; Alderman et al., 2001 and Case et al., 2005) and that children born underweight and with other health problems are likely to receive less schooling (Case et al., 2005 and Black et al., 2005).

\subsection{The contribution of our paper}

Our paper intends to contribute originally to this literature in several respects. First, we explicitly provide a framework for testing the productive and allocative theories on a cross-country sample including 19 European countries. Second, we propose instruments and robust econometric techniques in order to control for endogeneity and disentangle the direct effect of education years 
on health from the spurious correlation caused by omitted unobservable third drivers. More specifically we use birth quarters and average education years of the neighbouring regions as relevant and valid instruments since they are assumed (and indeed do) affect the respondent's education years while not directly her/his health status. Third, we provide further evidence for European countries on an anomaly already observed by Cutler and Lleras-Muney (2006) in the US on the impact of education on cancer. Our findings provide support for both the allocative and the productive theory at European level but also confirm the "anomaly" already observed by Cutler and Lleras-Muney (2006) in the US showing that the probability of contracting cancer is higher for the more educated.

\section{Our hypotheses}

In this section we formulate theoretical hypotheses which will be tested in the empirical analysis which follows on the basis of the above described (productivity and allocation) theories in the education-health literature.

More specifically we outline the following two hypotheses:

$\mathrm{H}_{0 \mathrm{~A}}$ : there is no difference in terms of health inputs (smoking, drinking, vigorous physical activities, overweight/obese) between the more and less educated;

$\mathrm{H}_{0 \mathrm{~B}}$ : there is no difference in terms of health outputs (cancer, Parkinson, hypertension, diabetes, etc.) between the more and less educated.

Based on the different combinations of our results we may have the following outcomes:

1) Both $H_{O A}$ and $H_{O B}$ are not rejected.

If education does not make any significant difference in terms of both health inputs and outputs neither the productive nor the allocative theories are supported by our empirical evidence. 


\section{2) $H_{O A}$ is not rejected while $H_{O B}$ is rejected.}

If the more educated report better health outputs (without having significantly different inputs) only the productive theory is supported: even though the more educated use the same health inputs of the less educated their health outcomes are significantly different.

\section{3) Both $H_{O A}$ and $H_{O B}$ are rejected. $H_{O B}$ is rejected even when we control for health inputs}

The more educated are significantly different from the less educated in terms of both health inputs and outputs. In this case both the allocative and productive theories are supported if the difference is in the direction that the more educated have healthier life styles (drink and smoke less, practice more intensely physical activities and are in less proportion overweight) and better health outcomes (are less likely to suffer from the different pathologies considered in our analysis) even after controlling for differences in health inputs.

4) $H_{O A}$ is rejected. $H_{O B}$ is not rejected once we control for health inputs, while it is rejected if we do not control for health inputs

If rejection of $\mathrm{H}_{0 \mathrm{~A}}$ goes in the direction that the more educated have better health inputs (i.e. drink and smoke less, practice more intensely physical activities and are in lower proportion overweight), while not better health outcomes once we control for the level of input only the allocative theory is supported. The difference between the more and less educated in terms of health outcomes would be entirely explained in this case by the significant differences in health inputs. If, on other terms, the less educated use the same levels of health inputs adopting healthier life styles they would have exactly the same health outputs as the more educated. This scenario obviously requires as maintained assumption that health inputs impact significantly upon health. 


\section{Database description}

Data for the empirical analysis come from three waves of the Survey of Health, Ageing and Retirement in Europe (SHARE), a panel dataset on health, socio-economic status, and the social and family networks of more than 45,000 Europeans aged 50 and over. The survey covers the following 19 countries: Austria, Germany, Sweden, Netherlands, Spain, Italy, France, Denmark, Greece, Switzerland, Belgium, Israel, Czech Republic, Poland, Ireland, Hungary, Portugal, Slovenia and Estonia.

We provide variable legend in Table 1, while descriptive statistics of our sample in Table A2. The average number of education years in the sample is 10.50 , while more than half of respondents are retired (around 52 percent). Around 44 percent of the sample is above 69 years and 12 percent above 79 years. Around 58 percent of sample respondents is married or with regular partner. The average number of children is 2.2 while that of grandchildren 2.6.

Table 2 provides as well descriptive statistics for four categories of health indicators. A first group of variables includes subjective and composite health indicators such as: i) the respondent selfassessed health status (health_insat); ${ }^{2}$ ii) the CASP (casp) quality of life indicator based on four subscales measuring control, autonomy, pleasure and self-realization (the indicator ranges from 12 to 48); iii) the ADLA (Activities of Daily Living) indicator; ${ }^{3}$ iv) the IADLA (Instrumental Activities of Daily Living) indicator, ${ }^{4} \mathrm{v}$ ) an index of cognitive functions ( $n$ _wordsrecalled $) ;{ }^{5}$ vi) an

\footnotetext{
${ }^{2}$ The indicator scores are 1 for "excellent" health, 2 for "very good", 3 for "good", 4 for "fair" and 5 for "poor" health.

${ }^{3}$ The ADLA (Activities of Daily Living) index measures the self-evaluated skills in performing the following tasks: dressing, bathing or showering, eating and cutting up food, walking across a room and getting in or out of bed. The index (ranging from 0 to 5) is higher the higher the difficulties in performing these tasks due to the reduced respondent's mobility.

${ }^{4}$ The IADLA (Instrumental Activities of Daily Living) index is calculated by summing responses on the self-evaluated skills in performing the following tasks: telephone calls, taking medications and managing money. The index (ranging from 0 to 3 ) is higher in presence of higher difficulties in performing these tasks due to the reduced respondent's mobility.

${ }^{5}$ A list of ten words is communicated to the respondent and, after a fixed time, the number of words recalled is reported.
} 
index of mobility (mobilityind) ${ }^{6}$ vii) an index measuring numeracy skills (numeracy) viii) a dummy which takes value one for individuals reporting long-term health problems, illness, disability or infirmity (longtermillness); ix) the number of visits to the doctor in the last 12 months (n_doctorvisits).

The second group of variables considered concerns illnesses and, more specifically: i) high blood pressure or hypertension (hypertension); ii) heart attack including myocardial infarction or coronary thrombosis or any other heart problem including congestive heart failure (heartattack); iii) stroke or cerebral vascular disease (stroke); iv) cancer or malignant tumour, including leukaemia or lymphoma, but excluding minor skin cancers (cancer); v) Parkinson disease (Parkinson); vi) diabetes or high blood sugar (diabetes); vii) asthma (asthma); viii) Arthritis, including osteoarthritis, or rheumatism (arthritis); ix) osteoporosis (osteoporosis); x) stomach or duodenal ulcer, peptic ulcer (ulcer); xi) cataracts (cataracts); xii) hip fracture or femoral fracture (fracture).

A third group of variables measures symptoms and, more specifically: joint pain, heart trouble, swollen legs, sleeping problems, falling down, fear of falling down, breathlessness, persistent cough, dizziness, stomach or intestine, incontinence.

Descriptive evidence on health conditions in our sample (see Table A1 in the Appendix) documents that half of respondents (50.6 percent) report a long-term illness, 36.2 percent hypertension and around 5 percent a cancer. Only 24.8 percent report no illnesses.

\subsection{Descriptive evidence on education related differences in terms of health inputs and outputs}

\footnotetext{
${ }^{6}$ The index measures the self-assessed ability of performing the following tasks: walking across a room, walking 100 metres, climbing one flight of stairs and climbing several flights of stairs.
} 
We perform a preliminary descriptive investigation on the impact of education on health inputs and outputs based on ISCED (International Standard Classification of Education) 1997 classification. We identify four groups which we define as: "primary school" (the ISCED level 1 of primary education or first stage basic education); "secondary school” (the ISCED level 2 including lower secondary or second stage of basic education), "high school" (the ISCED level 3 of (upper) secondary education) and "bachelor" (the ISCED levels 5 and 6 of first stage of tertiary education and second stage of tertiary education).

Evidence provided in Table 2 suggests several directions. For most of the considered health outputs education seems to have a clear-cut impact: 16.8 and 13.8 percent in the primary and secondary school group respectively got a heart attack against only 10.01 percent in the bachelor group. The descriptive education-hypertension gradient is as well remarkable: 41.5 percent in the primary school, 37.8 in the secondary school, 34.6 in the high school and 30 percent in the bachelor group. Diabetes affects 15.1 percent in the primary school group, 11.09 in the secondary school group, 10.02 in the high school group and 8 percent in the bachelor group. In all of these four cases the difference in means between the lowest and the highest education group is highly significant at 95 percent level. Findings on almost all the other pathologies considered (cataracts, asthma, arthritis, osteoporosis, Parkinson, stroke, etc.) register similar patterns. Indications are however not so clear cut and in the same direction for cancer: 3.99 percent of the primary school group has contracted a type of cancer against 4.5 percent in the secondary school and 5.4 percent in the bachelor group. ${ }^{7}$

Education seem to matter a lot also in terms of health inputs providing preliminary evidence in favour of the allocative theory hypothesis. The share of non drinkers in the last three months before the interview among those with primary school is 19.4 percent against 22 percent in the bachelor

\footnotetext{
${ }^{7}$ Using for those illnesses where the education effect is stronger an approach which is standard in the clinical literature we find that the risk of diabetes, hypertension and heart attack on an average follow-up of 42 months on our sample of are respectively 18.7 percent, 11.5 percent and 6.1 percent. The relative risk for those with no more than elementary school vis-à-vis those who did at least high school for diabetes, hypertension and heart attack are respectively 1.69, 1.47 and 1.99 .
} 
group. Of course drinking alcohol every day is not necessarily an unhealthy habit if one drinks moderately but, since we do not have more accurate measures of higher drinking intensity in our sample we cannot shed light further on this point. Average body mass index is also higher for the less educated (27.13 percent in the primary school group against 26.02 percent in the bachelor). ${ }^{8}$ Behind this aggregate result we find that the share of individuals who are obese (BMI above 34.9) is 22.4 percent in the primary school group, 21.1 in the secondary school group, 19.1 in the high school group and 13.6 in the bachelor group. Our descriptive findings also document that 54.8 percent of individuals in the primary school group hardly ever or never practice a sport, against 34.7 percent in the bachelor group. The only health style variable where the effect of education is less clear-cut is smoking. We find that smokers are 16.1 percent among the primary school group, 21.2 percent in the secondary school group, 22 percent in the high school group and 17.5 percent in the bachelor group. To conclude with, those with higher education have healthier life styles (mostly in terms of physical activity and diet with the notable exception of smoking habits).

The impact of education on physical functionalities from a descriptive point of view is also strong as expected. The mobility index is on average 82.3 for the primary school group, 57 for the secondary school, 41.03 for the high school and 30.6 for the bachelor group. The ADLA indicator registers a similar pattern with a value of 34.3 for the primary school group, 20.09 for the secondary school, 15.7 for the high school and 11.6 for the bachelor group. The distance between the low and high educated in terms of mental functionalities, where education is expected to produce a positive and significant impact per se, is relevant. In the word recall test performed by SHARE investigators individuals in the primary school group remember 4.06 words on average, against 4.90 in the secondary school group, 5.40 in the high school group and 5.84 in the bachelor group. The outcome

\footnotetext{
${ }^{8}$ The Body Mass Index is calculated as the weight divided by the square of height expressed in meters. 24.9 is conventionally considered a regular weight value. A BMI lower than 18 (higher than 29.9) is considered underweight (overweight). Obesity starts with BMI above 34.9. Withlock et al. (2009) find that individuals with BMI equal to 29 lose one year of life, while those with BMI to 31 two years of life.
} 
in the numeracy score of the primary school group is 2.72 , against 3.19 of the secondary school, 3.59 of the high school and 3.90 of the highest education group.

Consistently with what observed above in terms of health inputs, outputs and functionalities health (un)satisfaction falls with education. The primary school group registers an average (un) satisfaction level of 3.27 (health satisfaction between "fair" and "good"), the secondary school group of 2.98, the high school group of 2.88, while the bachelor group of 2.55 (health satisfaction between "good" and "very good"). Last but not least, the less educated go systematically more to the doctor (8.02 visits per year for the primary school group against 5.57 for the bachelor group). The interpretation of this indicator is ambiguous because, on the one side, doctor visits can be considered an input (patients may go to doctors for prevention) while, on the other side, a signal of bad health output (individuals with more or more severe pathologies are highly likely to go more to the doctor).

To conclude with, our descriptive evidence documents that the more educated have a different health input mix consisting in healthier life styles, better functionalities and better health outcomes in most pathologies (hearth attack, hypertension, diabetes and Parkinson) with the notable exception of cancer.

\section{Econometric findings}

Descriptive findings provided preliminary answers to interpret the stylized facts of differences in life expectancy among the less and the more educated. They cannot help us to understand however whether their more favourable health outcomes in many pathologies are determined by a direct effect of education or by an indirect effect which is mediated by income or life styles. The econometric analysis in first differences which follows may help us to answer to this question 
testing whether the impact of education on a given pathology is significant even when we control for standard socio-demographic factors and health styles.

More specifically, and in order to test hypotheses $\mathrm{H}_{0 \mathrm{~A}}$ and $\mathrm{H}_{0 \mathrm{~B}}$ outlined in section 2 our baseline specifications are

$$
\begin{aligned}
& \text { Health_indicator }_{i, t} \\
& \qquad \begin{array}{l}
= \\
\qquad+\beta \text { EduYears }_{i, t}+\gamma \text { Ln_Income }_{i, t}+\sum_{k=1}^{K} \delta_{k} \text { Socio_Dem }_{i, t} \\
\\
+\sum_{l=1}^{L} \lambda_{l} \text { HealthStyles }_{i, t}+\sum_{v=1}^{V} \chi_{v} \text { DInt_Year }_{i, v}+\sum_{g=1}^{G} \kappa_{g} \text { DCountry }_{i, g}+\varepsilon_{i, t}
\end{array}
\end{aligned}
$$

and

$$
\begin{aligned}
& \text { HealthStyle }_{i, t} \\
& =\alpha+\beta \text { EduYears }_{i, t}+\gamma \text { Ln_Income }_{i, t}+\sum_{k=1}^{K} \delta_{k} \text { Socio_Dem }_{i, t} \\
& +\sum_{v=1}^{V} \chi_{v} \text { DInt_Year }_{i, v}+\sum_{g=1}^{G} \kappa_{g} \text { DCountry }_{i, g}+\varepsilon_{i, t}
\end{aligned}
$$

where the dependent variable in (1), Health_indicator ${ }_{i, t}$ is one of the different health indicators being part of the synthetic indicators or specific illnesses described in the previous section. The impact of education on changes in health is captured by the variable $E d u Y e a r s_{i, t}$, beyond Ln_income (the log of per capita total household income), the vector of Socio_Dem regressors includes variables such as gender, age, employment and marital status, number of children and grandchildren. Health styles are measured by information on physical activities, alcohol, overweight/obesity and smoking habits (variables Sport, Alcohol, Overweight/Obese and Smoking respectively). All estimates include year (DInt_year) as well as country fixed effects (DCountry). The well-known problem of missing variables in the SHARE database for variables such as income 
is solved, as in most empirical studies using the same information, with the Christelis (2011) imputation method. ${ }^{9}$ All estimates are clustered at country level.

With reference to our hypothesis testing, $\mathrm{H}_{0 \mathrm{~A}}$ (no difference in health inputs) is not rejected if $\beta$ is not statistically different from zero in (2) and $\mathrm{H}_{0 \mathrm{~B}}$ (no difference in health outputs) is not rejected if $\beta$ is not statistically different from zero in (1). The allocative theory is supported by our empirical evidence if $\beta=0$ is rejected in (2), while the productive theory is supported if $\beta=0$ is rejected in (1). We as well obtain evidence that only the allocative theory works if $\beta=0$ is rejected in (2) but not in (1) and health styles impact significantly on health outcomes (or when education impacts significantly on health outcomes when not controlling for health styles).

Econometric findings presented in Table 3 document that lagged education years are significantly correlated with health inputs (life styles) consistently with the allocative theory. The more educated drink less (columns 1-2), practice more frequently vigorous physical activities (columns 3-4), smoke less (columns 5-6) and are in a less proportion overweight (columns 7-8).

Results presented in Table 4 (columns 1-4) document that all the descriptive evidence discussed above in terms of physical and mental functionalities, health satisfaction and doctor visits is confirmed when we control for concurring factors in econometric estimates. ${ }^{10}$ Education years are inversely correlated with health (un)satisfaction and doctor visits, while positively correlated with all the considered functionalities. In the same Table 4 we find that education years are negatively correlated with the insurgence of almost all pathologies, controlling or not for life styles. The impact of education years on cancer remains however positive confirming what already evidenced

\footnotetext{
${ }^{9}$ Missing information is imputed using the Fully Conditional Specification method (FCS) (Van Buuren et al., 2006). Conditionally on the non-missing values of other dataset variables a distribution for the missing value of a given variable is generated. Based on this approach five different imputed datasets (one for each iteration) are made available from the SHARE website. The variables in our empirical analysis with imputed values are number of children, logincome, number of grandchildren, limited activities. For each of them we use an average of values from the five datasets.

${ }^{10}$ Full estimate findings are presented in Tables A3a-A4d.
} 
in our descriptive findings. Overall our results provide support to both the allocative and productive theories.

In Table 4 (columns 5-8) we repeat the previous specifications correcting for attrition bias. The problem of attrition is particularly relevant in empirical health studies not using mortality rates and in our SHARE sample with aged respondents who may exit from the survey for various reasons (death or nonresponse due to health worsening or other reasons unrelated to health). To check whether our findings are robust to attrition bias we follow the standard approach of regressing the probability of survival on the set of controls used in our specifications and use the inverse of the predicted probability as a weight of our estimates. ${ }^{11}$ The impact of education years is is stronger and robust on weight and drinking, while no more significant on several pathologies. The effects which remain significant are those on pathologies which are presumably more influenced by life styles such as heart attack, stroke, diabetes, arthritis and osteoporosis. Note as well that hypertension remains significant only when not corrected for life styles. This implies that, once we correct for attrition bias, the impact of education on hypertension is explained by the allocative and not by the productive theory. The finding is consistent with what found by Goldman and Lakdawalla (2002) documenting that the introduction of more user-friendly drugs such as beta blockers reduced the education-health gradient (and the dependence of health output from the higher ability of the more educated of using properly prescribed therapies).

The above mentioned theoretical findings are consistent and provide a reasonable explanation for the reported result on health satisfaction. Superior physical and mental functionalities and a lower

\footnotetext{
11 The attrition probability is estimated as follows $A_{i, t}=\alpha+\sum_{k=1}^{K} \beta_{k}$ Sociodem $_{i, t}+\gamma$ noconditions $_{i, t}+\delta$ nosymptoms $_{i, t}+\sum_{v=1}^{V-1} \chi_{v}$ DInt_Year $_{i, v}+\sum_{g=1}^{G-1} \kappa_{g}$ DCountry $_{i, g}+\epsilon$ where $A_{i, t}$ is a dummy variable $=1$ if the respondent is not in the panel for at least 2 waves, Sociodem variables include gender, age, income, employment and marital status, education years, number of children and grandchildren, smoking, drinking and physical activity habits and a dummy for respondents being overweight or obese; nocondition and nosymptoms are dummy variables taking value one if the respondent reports not having specific illnesses or symptoms respectively. Results from the attrition estimates (omitted for reasons of space) show that female gender, the number of grandchildren and the nocondition dummy impact negatively on attrition, while being divorced/separated and doing sport activities infrequently impact positively. This evidence strongly suggests that mortality and worsening health conditions are likely to be the main cause of nonresponses. For a similar approach on the attrition weighting procedure see, among others, Raab et al. (2005), Nicoletti and Peracchi (2005) and Vandecasteele and Debels (2007).
} 
number of chronic diseases justify why we find education years impacting positively on health satisfaction. In this sense findings on objective health indicators provide a validity check of those on the subjective health indicator.

Overall our econometric findings may be interpreted in the sense that higher education leads to better health styles and functionalities with positive consequences on health. In addition to it, even after controlling for health styles, the more educated have a better technology (i.e. higher ability to exploit medical information) which allows them to obtain better health outcomes even after controlling for the input mix. Overall, these findings provide a reasonable and empirically grounded interpretation of what is behind the stylized fact of the positive correlation between education years and life expectancy.

In terms of economic significance our results indicate that one year more of education reduces by around 0.3 percent the probability of incurring in a long term illness, by 0.2 percent in a heart attack, by 0.1 percent in a stroke, by 0.27 percent in diabetes, by 0.4 percent in arthritis and by 0.14 percent in osteoporosis, while raising by 0.1 percent that of incurring in cancer. If we consider that the education year distance between those with primary school and those with tertiary education may be up to 11-13 years we find that the magnitude of the effect between the primary and the bachelor group becomes relevant (i.e. around 4 percent higher probability of incurring in a long term illness).

Note finally that, even before controlling for endogeneity, the significant correlation we find in Tables 3-4 is important per se. It means that if we observe a highly educated individual such individual has a higher health outcomes either because education causes such outcomes or because there are other characteristics which are associated to education and good health outcomes. In both cases higher educated should expect a better life outcome. The policy suggestion however is different since in the first case we must promote education to have better health, while in the second 
case it is the unobservable driver related to education that should be possibly supported by proper policies.

This is why is important to use instrumental variables to verify whether the observed correlation hides a causality link from education years to health styles, health outcomes and functionalities.

\section{Instrumental Variable estimates}

To control whether our findings hide a direct causality nexus between education, on the one side, and life styles, health outcomes and functionalities, on the other side, we perform IV estimates using the following set of equations

$\operatorname{EduYears}_{i, t}=\zeta+\xi Z_{i, t}+\eta_{i, t}$

Health_indicator ${ }_{i, t}$

$$
\begin{aligned}
& =\alpha+\beta E d \widehat{\text { YYears }}_{i, t}+\gamma \text { Ln_Income }_{i, t}+\sum_{k=1}^{K} \delta_{k} \text { Socio_Dem }_{i, t} \\
& +\sum_{l=1}^{L} \lambda_{l} \text { HealthStyles }_{i, t}+\sum_{v=1}^{V} \chi_{v} \text { DInt_Year }_{i, v}+\sum_{g=1}^{G} \kappa_{g} \text { DCountry }_{i, g}+\varepsilon_{i, t}
\end{aligned}
$$

where equations (2a) and (2b) represent respectively the first and second stage of our IV estimation. Our identification strategy consists of two instruments ( $Z$ ) for $\operatorname{EduYears}_{i, t}$ in (1). Following Angrist and Krueger (1991), we instrument education years with the respondent's quarter of birth (First_qtr $r_{i}$ is a dummy equal to one for those born in the first quarter of the year) by exploiting the longer/shorter stay at school due to the month of birth and the local laws regulating the entry age and the length of compulsory schooling. The exclusion restriction hinges on the exogenous variation in $E d u Y$ ears $_{i, t}$ induced by the respondent's month of birth which is correlated with our outcome variable (health) only through schooling years. The validity of the instrument can be 
hardly confuted since month of birth cannot be suspected of affecting directly health outcomes. The relevance of the instruments hinges on the correlation between birth quarter and education years. Such correlation is expected since i) students born in different periods of the year start school at different ages and ii) compulsory schooling laws require students to attend school up to a specific age. Students born early in the year (i.e. January) are expected (net of those who anticipate) to be on average older when they enrol (i.e. typically in September) relative to those who are born later in the year. This implies that, by reaching the legal dropout age earlier, the former are more likely to have attended the school for a shorter period than the latter. Or, on the contrary, by reaching the end of a given course of studies later, they will have coeteris paribus a lower propensity to start the following education cycle. Figure 1 reports the k-density of schooling years by quarter of births for the first wave of SHARE respondents. As expected, the distributions differ especially at middle values of education years with fewer respondents reporting 8 or 13 years of schooling if born in the first quarter vis-à-vis those born later in the year. From a statistical point of view, the two-sample Kolmogorov-Smirnov test confirms that education years are distributed in a statistically different way by quarter of birth ( $\mathrm{p}$-value $=0.006$; combined $\mathrm{K}-\mathrm{S}=0.0269)$. The negative effect of being born in the first quarter of the year (relative to being born later in the year) on education years is also confirmed by results from first stage IV regressions discussed below.

The second instrument, $\overline{\text { EduYears }}_{i, t}$, is the average number of education years in the $i$ 's neighbouring region. In presence of multiple neighbours we use a weighted average of $\overline{\operatorname{EduYears}}_{i, t}$ for each neighbouring region, with the weights equal to the length of the shared border. ${ }^{12}$ We believe that the instrument is relevant due to peer effects and/or social conformity. ${ }^{13}$

\footnotetext{
12 The instrumental variable is derived by choosing the second level of the NUTS (Nomenclature of Territorial Units for Statistics) subdivision as calculation units. Missing values at second NUTS level were replaced with information at first (Germany) or third (Denmark and Estonia) while records with missing information at both second and third NUTS levels were discarded. The variable is built by using the GIS (Geographic Information System) framework. NUTS adjacent to the residence are identified for each record and lengths in $\mathrm{km}$ of the shared boundaries between the residence NUTS and each of the adjacent ones are calculated. Values of the corresponding non-instrumental parameter detected in each adjacent NUTS are subsequently averaged using the respective length of the shared boundary as a weight. In case of no-neighbors NUTS (i.e. islands), values of the instrumental variable detected in the closest NUTS have been considered. Routes within the same nation have been considered as better means of communication than
} 
We also believe our exclusion restriction is valid, since it is plausible for $\overline{\operatorname{EduYears}}_{i, t}$ to affect changes in health indicators not directly but only indirectly through EduYears E $_{i, t} \cdot{ }^{14}$

Estimation findings obtained by instrumenting schooling years with First_qtr $_{i}$, with $\overline{\operatorname{EduYears}}_{i, t}$, and with both simultaneously, are reported in columns 1-4, 5-8 and 9-14 of Table 5 respectively. ${ }^{15}$ IV results generally confirm the main findings in Table 3 concerning functionalities. The positive effects of education years remain significant for all the functionality indicators considered but the number of doctor visits (as in Table 3 when controlling for attrition) and the number of chronic diseases. Different results, however, emerge when considering specific illnesses. When using First_qtr ${ }_{i}$ as single instrument, education years negatively impact the likelihood of reporting most of the specific illnesses. Conversely, when we use $\overline{\operatorname{EduYears}}_{i, t}$ or both as instruments, baseline results are confirmed only for hypertension and cancer. Since the IV coefficients when using $\overline{\operatorname{EduYears}}_{i, t}$ or both instruments look closer in magnitude to the baseline ones in Table 3, we consider them as more reliable with respect to the estimation results where First_qtr ${ }_{i}$ is used as a single instrument.

To summarize, the effect of schooling years on health functionalities is generally robust also to the IV robustness checks, while it stays so only for hypertension (one education years reduces by 0.46

\footnotetext{
international ones and adjacent or closest NUTS belonging to the respondent's nation have received a $75 \%$ more weighted value in the calculation of the mean. Instrumental variables were generated by using cartographical data provided by "Eurostat". Two different sets of cartographical data had to be used, i.e. the administrative units of 2003 (for Greece, Italy, Denmark, Estonia, Sweden and Germany) and of 2010 (for the other countries) since some countries changed names, number and geographic boundaries of their NUTS during the time period considered in our sample. All calculations were done using the R 3.1.1 software and the add-one packages "maptools", "rgeos", "spdep" and "doParallel". For further reference in the literature on the use of geographical aggregates as internal instruments see also Aslam and Corrado (2012).

${ }^{13}$ The rationale for the relevance of our instrument hinges on the literature of peer effects and/or social conformity since we interpret $\overline{E d u Y e a r s}_{i, t}$ as out-group effects calculated as the average of the responses across all 'neighboring' groups/regions by the $i$ 's region. In this sense we extend the work by Cohen-Cole (2006) by considering between-group interdependencies where a group is defined by individuals living in a specific region. Our identification strategy is based on the fact that agents' schooling years in one region are correlated with those of other agents in the surrounding regions and guaranteed if the number of individuals exceeds the number of regions (which is our case).

${ }^{14}$ Notice that $\overline{\text { EduYears }}_{i, t}$ is not significant when added as a control in the specifications in Tables 3a-3c. Results from these estimates are omitted for reason of space but available upon request.

${ }^{15}$ Full estimate findings are provided in Tables A5a-A5e in the Appendix.
} 
percent the insurgence of the pathology) and cancer (one education years increases by 0.26 percent the insurgence of the pathology) when considering specific illnesses.

\subsection{Further discussion on our results}

In what presented above we find support for allocative and productive theories confirming that education has positive effects on physical and mental functionalities and on health outcomes, with or without controlling for health styles and after controlling for attrition bias. Some of the effects on specific illnesses are no longer significant when using our IV approach, while a causality nexus going from education to outputs is verified only for hypertension and cancer. The paradox on the effect of education on cancer is therefore supported by our research confirming what already observed by Cutler and Lleras-Muney (2006). We provide some tentative rationales for the paradox.

The first is that the more educated die less of other diseases and therefore are more likely to get cancer. This rationale however does not seem supported by our data since, when considering cancer patients, the more educated are in generally older than the less educated. A second rationale is that illness reporting accuracy is higher among the more educated. This would not explain however why in all other illnesses the more educated perform better and would apply only if the reporting inaccuracy of the less educated applies only to cancer. Or it can explain it if we argue that reporting inaccuracy is higher for diseases with no symptoms such as cancer in its first stages. A third rationale is that unobservable health style components associated with the life styles of the more educated are positively correlated with cancer (i.e. late marriage and lower birth rate for women may be related to breast cancer, more sedentary life and higher stress of the high educated work to other forms, etc.). The puzzle remains since we do not find a univocal conclusive and clear cut explanation. 


\section{Conclusions}

Our empirical work investigates on a large sample of Europeans aged above 50 whether the different life expectancy of the more and less educated found in the literature may be accounted for by differences in health styles and outcomes. We document that, consistently with the allocative theory, the more educated adopt significantly healthier life styles (with the notable exception of smoking habits). However, the productive theory is as well supported by our evidence since, even after controlling for health styles, the more educated have significantly better functionalities (mobility and memory skills) and significantly lower probability of incurring in chronic diseases. A paradox common to the recent evidence provided from Cutler and Lleras-Muney (2006) for the US is that the more educated have a higher probability of contracting cancer. We find a similar result in our analysis.

Some but not all the documented effects are robust when controlling for endogeneity with IV estimates. More specifically a causal link is found for physical and mental functionalities, hypertension and cancer. Overall, these effects are largely consistent with the reported positive impact of education years on health satisfaction and provide a sound interpretation of the stylized facts of the higher life expectancy of the more educated. On this last point our findings predict that the education - life expectancy gap will get larger in case of a relatively larger progress in cancer treatments vis-à-vis other pathologies. 


\section{References}

Alderman, H., Behrman, J.R., Lavy, V. and Menon, R. (2001). "Child health and school enrollment: a longitudinal analysis". Journal of Human Resources, 36, 185-205.

Arendt, J.N. (2005). "Does Education cause better health? A panel data analysis using school reform for identification." Economics of Education Review, 24, 149-160.

Aslam, A. and Corrado, L. (2012). "The Geography of Well-Being." Journal of Economic Geography, 12(3), 627-649.

Becker, G.S. (1965). “A theory of the allocation of time.” Economic Journal, 75, 493-517.

Behrman, Jere and Rosenzweig, Mark R., The Returns to Increasing Body Weight (February 2001). PIER Working Paper No. 01-052.

Black, S.E, Devereux, P.J. and Kjell Salvanes. (2005). "From the Cradle to the Labor Market? The Effect of Birth Weight on Adult Outcomes." NBER working paper n. 11796.

Braakmann, N. (2010). "The causal relationship between education, health and health related behaviour: Evidence from a natural experiment in England." Journal of Health Economics, vol. 30, n. 4, July 2011, Pages 753-763,

Card, D. (1999). The causal effect of education on earnings. In Ashenfelter, O. and Card, D. (Eds.), Handbook of Labor Economics, vol. 3. Elsevier, Amsterdam, 1801-1863.

Card, D. (2001). "Estimating the return to schooling: progress on some persistent econometric problems." Econometrica, 69, 127-1160.

Case, A., Fertig, A. and Paxson, C. (2005). "The Lasting Impact of Childhood Health and Circumstance." Journal of Health Economics, 24, 365-389.

Chaikind, S. and Corman, H. (1991). "The impact of low birthweight on special education costs". Journal of Health Economics 10, 291-311.

Christelis, D. (2011). "Imputation of missing data in Waves 1 and 2 of SHARE". CSEF Working Paper n. 278.

Christenson, B. and Johnson, N.E. (1995). "Educational Inequality in Adult Mortality: An Assessment with Death Certificate Data from Michigan,” Demography, May, 32.

Cohen-Cole, E. (2006). “Multiple Groups Identification in the Linear-in-Means Model.” Economics Letters, n. 92, 157-162.

Corsini, V. (2010). "Highly educated men and women likely to live longer. Life expectancy by educational attainment." Eurostat, Statistics in Focus, 24.

Currie, J. (2000). Child health. In Culyer, A.J. and Newhouse, J.P. (Eds.), Handbook of Health Economics, vol. 1B. Elsevier, Amsterdam, 1053-1090. 
Currie, J. and Moretti, E. (2003). "Mother's Education and the Intergenerational Transmission of Human Capital: Evidence from College Openings." Quarterly Journal of Economics, n. 4, nov. 2003, 1495-1532.

Cutler, D. and Lleras-Muney, A. (2006). "Education and Health: Evaluating Theories and Evidence." NBER working paper, n. 12352.

Deaton, A. and Paxson, C. (2001). Mortality, education, income, and inequality among American cohorts. In Wise, D.A. (Ed.), Themes in the Economics of Aging. University of Chicago Press, Chicago, IL, 129-165.

Edwards, L.N. and Grossman, M. (1979). The Relationship between children's health and intellectual development. In Mushkin, S.J. and Dunlop, D.D. (Eds.), Health: What Is It Worth. Pergamon Press, Elmsford, NY, 273-314.

Elo, I.T. and Preston, S.H. (1996). "Educational Differentials in Mortality: United States 1979-85." Social Science and Medicine, 42, 47-57.

Erbsland, M., Ried, W. and Ulrich, V. (1995). "Health, health care, and the environment. Econometric evidence from German micro data." Health Economics, 4, 169-182.

Fingleton, B. and Corrado, L. (2012). "Where is the Economics in Spatial Econometrics?" Journal of Regional Science, 52(2), 210-239.

Gilleskie, D.B. and Harrison, A.L. (1998). "The effect of endogenous health inputs on the relationship between health and education". Economics of Education Review 17, 279.297.

Goldman, D. and Lakdawalla, D. (2001). "Understanding health disparities across education groups." NBER working paper n. 8328.

Goldman, D. and Lakdawalla, D. (2002). "Health disparities and medical technology". RAND Working Paper.

Grossman, M. (2006). Education and Nonmarket Outcomes. In Handbook of the Economics of Education, Hanushek, E. and Welch, F. (Eds), North Holland, Amsterdam, 577-633.

Kenkel, D. (1991) "Health Behavior, Health Knowledge and Schooling." Journal of Political Economy, April, 99(2).

Kenkel, D., Lillard, D. and Mathios, A. (2006). "The roles of high school completion and GED receipt in smoking and obesity." Journal of Labor Economics, 24, 635-660.

Kunst, A.E. and Mackenbach J.P. (1994). "The Size of mortality differences associated with educational level in nine industrialized countries." American Journal of Public Health, vol 84, 6, 932-037.

Lleras-Muney, A. (2005), "The Relationship Between Education and Adult Mortality in the United States." Review of Economic Studies, 72, 189-221. 
Liang, J., McCarthy, J.F., Jain, A., Krause, N., Bennett, J.M. and Gu, S. (2000). "Socioeconomic gradient in old age mortality in Wuhan, China." Journals of Gerontology. Series B, Psychological Sciences and Social Sciences, 55, 222-233.

Lynch, S.M. (2003). "Cohort and Life Course Patterns in the Relationship Between Education and Health: A Hierarchical Approach.” Demography, 40(2), 309-331.

Manor, O., Eisenbach, Z., Peritz, E. and Friedlander, Y. (1999). "Mortality Differentials among Israeli Men.” American Journal of Public Health, December, vol. 89, n. 12, 1807-1813.

Meara, E. (2001). "Why is Health Related to Socio-Economic Status? The Case of Pregnancy and Low Birth Weight." NBER working Paper n. 8231.

Meara, E.R., Richards, S. and Cutler, D.M. (2008). "The gap gets bigger: changes in mortality and life expectancy, by education, 1981-2000.” Health Affairs, 27(2), 350-60.

Mustard, C.A., Derksen, S., Berthelot, J.M., Wolfson, M. and Roos, L.L. (1997). "Age Specific education and income gradient in morbidity and mortality in a Canadian province." Social Science and Medicine, vol. 45, n. 3, 383-397.

Nicoletti, C. and Peracchi, F. (2005). "Survey response and survey characteristics: microlevel evidence from the European Community Household Panel." Journal of the Royal Statistical Society A, 168, 763-781.

Olshansky, S.J., et al. (2012). "Differences In Life Expectancy Due To Race And Educational Differences Are Widening, And Many May Not Catch Up." Health Affairs, 31, n. 8, 1803-1813.

Oreopoulos, P. (2007). "Do dropouts drop out too soon? Wealth, health and happiness from compulsory schooling." Journal of Public Economics, vol. 91, issues 11-12, 2213-2229.

Raab, G., Purdon, S. and Buckner, K. (2005). Practical Exemplars and Survey Analysis, URL: 5http://www.napier.ac.uk/depts/fhls/peas/4

Rosenzweig, M.R., and Schultz, T.P. (1983). "Estimating a household production function: Heterogeneity, the demand for health inputs, and their effects on birth weight". Journal of Political Economy, 91, 723-746.

Ross, C.E. and Mirowsky, J. (1999). "Refining the association between education and health: The effects of quantity, credential, and selectivity.” Demography, 36, 445-460.

Ross, C.E. and Wu, C. (1995). "The Links Between Education and Health." American Sociological Review, 60, 719-45.

Van Buuren, S., Brand, J.P.L., Groothuis-Oudshoorn, C.G.M. and Rubin, D.B. (2006). "Fully conditional specification in multivariate imputation." Journal of statistical computation and simulation, 76 (12), 1049-1064.

Vandecasteele, L. and Debels, A. (2007). "Attrition in Panel Data: The Effectiveness of Weighting." European Sociological Review , 23 (1), 81-97. 
Wagstaff, A. (1986). "The demand for health: some new empirical evidence." Journal of Health Economics, 5, 195-233.

Whitlock, G., Lewington, S., Sherliker, P., Clarke, R., Emberson, J., Halsey, J., Qizilbash, N., Collins, R. and Peto, R. (2009). "Body-mass index and cause-specific mortality in 900000 adults: collaborative analyses of 57 prospective studies." Lancet Prospective Studies Collaboration., 28, 373, 1083-1096.

Table 1 - Variable Legend

\begin{tabular}{ll}
\hline \multicolumn{1}{c}{ Variable } & \multicolumn{1}{c}{ Description } \\
\hline N_Doctorvisits & how often seen or talked to medical doctor last 12 months \\
N_Chronicdeseases & number of chronic diseases \\
Adla & activities of daily living index (high: has difficulties) (see section 2) \\
ladla & instrumental activities of daily living index (high: has difficulties) (see section 2) \\
Mobilityind & mobility index (high: has difficulties) \\
N_Wordsrecalled & results of word recalling task (see section 2) \\
Numeracy & numeracy score: mathematical performance (see section 2) \\
Casp & CASP: quality of life and well-being index (see section 2) \\
Health_Insat. & self-perceived health - us version (1 = excellent; $2=$ very good; $3=$ good; $4=$ fair; $5=$ poor) \\
Longtermillness & Dummy variable=1 if the individual has a long-term illness \\
Heartattack & Dummy variable=1 if the doctor told you had: heart attack. 0 otherwise
\end{tabular}


Hypertension

Stroke

Diabetes

Asthma

Arthritis

Osteoporosis

Cancer

Ulcer

Parkinson

Cataracts

Fracture

Noconditions

Otherconditions

Female

Eduyears

Married

Reg_Partnership

Separated

Never_Married

Divorced

Widowed

Retired

Employed

Unemployed

Housework

Other_Job

N_Children

N_Grandchildren

Drinking

\section{Sports}

Smoking

Bmi

OverWeight_Obese

Country

First_Qtr

EduYears

Lnincome
Dummy variable $=1$ if the doctor told you had: high blood pressure or hypertension. 0 otherwise

Dummy variable $=1$ if the doctor told you had: stroke. 0 otherwise

Dummy variable $=1$ if the doctor told you had: diabetes or high blood sugar. 0 otherwise

Dummy variable $=1$ if the doctor told you had: asthma. 0 otherwise

Dummy variable $=1$ if the doctor told you had: arthritis. 0 otherwise

Dummy variable $=1$ if the doctor told you had: osteoporosis. 0 otherwise

Dummy variable $=1$ if the doctor told you had: cancer. 0 otherwise

Dummy variable $=1$ if the doctor told you had: stomach or duodenal ulcer, peptic ulcer. 0 otherwise

Dummy variable $=1$ if the doctor told you had: Parkinson disease. 0 otherwise

Dummy variable $=1$ if the doctor told you had: cataracts. 0 otherwise

Dummy variable $=1$ if the doctor told you had: hip fracture or femoral fracture. 0 otherwise

Dummy variable $=1$ if the doctor told you had: none. 0 otherwise

Dummy variable $=1$ if the doctor told you had: other conditions. 0 otherwise

Dummy variable $=1$ if the respondent's gender is female. 0 otherwise

years of schooling

Dummy variable $=1$ if the respondent is married. 0 otherwise

Dummy variable $=1$ if the respondent has a registered partnership. 0 otherwise

Dummy variable $=1$ if the respondent is separated. 0 otherwise

Dummy variable $=1$ if the respondent has never been married. 0 otherwise

Dummy variable $=1$ if the respondent is divorced. 0 otherwise

Dummy variable $=1$ if the respondent is widowed. 0 otherwise

Dummy variable $=1$ if the respondent is retired. 0 otherwise

Dummy variable $=1$ if the respondent is employed. 0 otherwise

Dummy variable $=1$ if the respondent is unemployed. 0 otherwise

Dummy variable $=1$ if the respondent deals with housework .0 otherwise

Dummy variable $=1$ if the respondent has a second job. 0 otherwise

number of children

number of grandchildren

Frequency of alcohol consumption in the last 3 months (0/1 dummies): Almosteveryday, 5or6daysaweek, 3or4daysaweek, Onceortwiceaweek, Onceortwiceamonth, Lessoftenthanonceamonth,

Notatallinthelastthreemonths

Frequency of sports or vigorous activities (0/1 dummies): Min1week, Oneweek, OneorThreemonth, Hardly_ever_never.

Dummy variable $=1$ if the respondent smokes at the present time

body mass index (easySHARE version)

Dummy variable $=1$ if the respondent is overweight $(29.9<\mathrm{BMl}<34.9)$ or obese $(\mathrm{BMI}>34.9) .0$ otherwise country identifier

See section 5

See section 5

Ln of household total gross income. Its value is equal to the sum over all household members of the individual-level values of: annual net income from employment and self-employment (in the previous year); Annual public old age/early or pre-retirement/disability pension (or sickness benefits); Annual public unemployment benefit or insurance, public survivor pension from partner; Annual war pension, private (occupational) old age/early retirement/disability pension, private (occupational) survivor pension from partner's job, public old age supplementary pension/public old age/public disability second pension, secondary public survivor pension from spouse or partner, occupational old age pension from a second and third job; Annual public and private long-term insurance payments; Annual life insurance payment, private annuity or private personal pension, private health insurance payment, alimony, payments from charities received; Income from rent. Values of the following household level variables are added: Annual other hhd members' net income; Annual other hhd members' net income from other sources; Household bank accounts, government and corporate bonds, stocks/shares; mutual funds. 
Table 2: Health styles, health outcomes and functionalities by education groups

\begin{tabular}{|c|c|c|c|c|c|}
\hline & Variable & $\begin{array}{l}\text { Primary } \\
\text { School }\end{array}$ & $\begin{array}{l}\text { Secondary } \\
\text { School }\end{array}$ & $\begin{array}{l}\text { High } \\
\text { School }\end{array}$ & Bachelor \\
\hline \multirow[t]{10}{*}{$\begin{array}{l}\text { Synthetic indicators and } \\
\text { functionality indexes }\end{array}$} & Casp & 34.822 & 36.554 & 37.918 & 38.970 \\
\hline & N_Chronicdeseases & 1.655 & 1.390 & 1.233 & 1.103 \\
\hline & N Doctorvisits & 8.024 & 6.971 & 6.316 & 5.573 \\
\hline & lälla & 0.157 & 0.075 & 0.047 & 0.038 \\
\hline & Adla & 0.343 & 0.209 & 0.157 & 0.116 \\
\hline & N_Wordsrecalled & 4.066 & 4.896 & 5.403 & 5.844 \\
\hline & Mobilityind & 0.823 & 0.570 & 0.413 & 0.306 \\
\hline & Numeracy & 2.719 & 3.190 & 3.593 & 3.902 \\
\hline & Heatlh_Insat_Lastwave & 3.274 & 2.976 & 2.880 & 2.549 \\
\hline & $\mathrm{Bmi}$ & 27.142 & 26.999 & 26.637 & 25.823 \\
\hline \multirow{14}{*}{ Specific illnesses } & Longtermillness & 0.553 & 0.528 & 0.482 & 0.440 \\
\hline & Limitedactivities & 2.266 & 2.334 & 2.445 & 2.544 \\
\hline & Heartattack & 0.168 & 0.138 & 0.113 & 0.101 \\
\hline & Hypertension & 0.415 & 0.378 & 0.346 & 0.300 \\
\hline & Stroke & 0.050 & 0.044 & 0.036 & 0.030 \\
\hline & Diabetes & 0.151 & 0.119 & 0.102 & 0.080 \\
\hline & Asthma & 0.040 & 0.028 & 0.024 & 0.027 \\
\hline & Arthritis & 0.294 & 0.213 & 0.186 & 0.158 \\
\hline & Osteoporosis & 0.072 & 0.052 & 0.037 & 0.036 \\
\hline & Cancer & 0.045 & 0.052 & 0.050 & 0.054 \\
\hline & Ulcer & 0.062 & 0.060 & 0.054 & 0.046 \\
\hline & Parkinson & 0.011 & 0.006 & 0.006 & 0.006 \\
\hline & Cataract & 0.105 & 0.077 & 0.065 & 0.068 \\
\hline & Fracture & 0.031 & 0.023 & 0.018 & 0.014 \\
\hline \multirow[t]{6}{*}{ Drinking } & 5or6days_A_Week & 0.094 & 0.101 & 0.109 & 0.090 \\
\hline & 3or4days_A_Week & 0.087 & 0.110 & 0.135 & 0.124 \\
\hline & 1or2_A_Week & 0.119 & 0.166 & 0.197 & 0.220 \\
\hline & 1or2_A_Month & 0.037 & 0.059 & 0.077 & 0.106 \\
\hline & $<1 \_$A_Month & 0.017 & 0.024 & 0.030 & 0.038 \\
\hline & 0_For_3_Months & 0.194 & 0.182 & 0.180 & 0.220 \\
\hline \multirow[t]{6}{*}{ Sport } & 1_Week & 0.114 & 0.124 & 0.148 & 0.167 \\
\hline & 10r3_A_Month & 0.088 & 0.083 & 0.094 & 0.095 \\
\hline & Hardlyever_Or_Never & 0.548 & 0.445 & 0.376 & 0.347 \\
\hline & Smoking & 0.161 & 0.212 & 0.220 & 0.175 \\
\hline & Overweight_Obese & 0.661 & 0.645 & 0.613 & 0.535 \\
\hline & Obese & 0.224 & 0.211 & 0.191 & 0.136 \\
\hline
\end{tabular}


Table 3. The determinants of health behavior

\begin{tabular}{|c|c|c|c|c|c|c|c|c|}
\hline Dep. Var: & $\begin{array}{c}1) \\
\text { Random } \\
\text { Effects } \\
\text { D }\end{array}$ & $\begin{array}{l}\text { (2) } \\
\text { Pooled OLS } \\
\text { (Attrition adj.) } \\
\text { IKING } \\
\end{array}$ & $\begin{array}{c}\text { (3) } \\
\text { Random } \\
\text { Effects }\end{array}$ & $\begin{array}{l}\text { (4) } \\
\text { Pooled OLS } \\
\text { (Attrition adj.) } \\
\text { ORT } \\
\end{array}$ & $\begin{array}{c}(5) \\
\text { Random } \\
\text { Effects } \\
\mathrm{S}\end{array}$ & $\begin{array}{l}\text { (6) } \\
\text { Pooled OLS } \\
\text { (Attrition adj.) } \\
\text { KING } \\
\end{array}$ & $\begin{array}{c}(7) \\
\text { Random } \\
\text { Effects } \\
\text { Overv }\end{array}$ & $\begin{array}{c}\text { (8) } \\
\text { Pooled OLS } \\
\text { (Attrition adj.) } \\
\text { ight_Obese }\end{array}$ \\
\hline Eduyears & $\begin{array}{l}0.0404^{* * *} \\
(0.00860)\end{array}$ & $\begin{array}{c}0.0724^{* * *} \\
(0.0128)\end{array}$ & $\begin{array}{c}-0.00911^{* *} \\
(0.00374)\end{array}$ & $\begin{array}{c}-0.0112 \\
(0.00701)\end{array}$ & $\begin{array}{l}-0.00254^{*} \\
(0.00133)\end{array}$ & $\begin{array}{l}-0.00524^{* *} \\
(0.00200)\end{array}$ & $\begin{array}{c}-0.0100^{* * *} \\
(0.00143)\end{array}$ & $\begin{array}{l}-0.0147^{* * *} \\
(0.00147)\end{array}$ \\
\hline Female & $\begin{array}{c}-1.178^{* * *} \\
(0.0955)\end{array}$ & $\begin{array}{c}-0.942^{* * *} \\
(0.187)\end{array}$ & $\begin{array}{l}0.225^{\star * *} \\
(0.0294)\end{array}$ & $\begin{array}{l}0.255^{\star * \star} \\
(0.0602)\end{array}$ & $\begin{array}{c}-0.0858^{* * *} \\
(0.0140)\end{array}$ & $\begin{array}{l}-0.0453 \\
(0.0298)\end{array}$ & $\begin{array}{l}-0.104^{* * *} \\
(0.0196)\end{array}$ & $\begin{array}{l}-0.110^{* * *} \\
(0.00930)\end{array}$ \\
\hline Age55_59 & $\begin{array}{c}0.0847^{* \star \star} \\
(0.0246)\end{array}$ & $\begin{array}{l}0.153^{\star * \star} \\
(0.0421)\end{array}$ & $\begin{array}{c}0.0637^{\star \star *} \\
(0.0130)\end{array}$ & $\begin{array}{c}0.0955^{\star \star \star} \\
(0.0235)\end{array}$ & $\begin{array}{l}-0.0325^{\star * *} \\
(0.00416)\end{array}$ & $\begin{array}{c}-0.0267^{\star \star} \\
(0.0114)\end{array}$ & $\begin{array}{l}0.0398^{\star * \star} \\
(0.00564)\end{array}$ & $\begin{array}{c}0.0120 \\
(0.00896)\end{array}$ \\
\hline Age60_64 & $\begin{array}{l}0.120^{\star *} \\
(0.0504)\end{array}$ & $\begin{array}{l}0.306^{\star \star \star} \\
(0.0688)\end{array}$ & $\begin{array}{l}0.136^{\star * \star} \\
(0.0261)\end{array}$ & $\begin{array}{l}0.164^{* \star *} \\
(0.0262)\end{array}$ & $\begin{array}{c}-0.0840^{* * *} \\
(0.00875)\end{array}$ & $\begin{array}{c}-0.0768^{* * *} \\
(0.0118)\end{array}$ & $\begin{array}{l}0.0505^{\star \star \star} \\
(0.00666)\end{array}$ & $\begin{array}{l}0.0227^{*} \\
(0.0108)\end{array}$ \\
\hline Age65_69 & $\begin{array}{c}0.0983 \\
(0.0613)\end{array}$ & $\begin{array}{c}0.192^{*} \\
(0.0948)\end{array}$ & $\begin{array}{l}0.209^{* * *} \\
(0.0316)\end{array}$ & $\begin{array}{l}0.215^{\star * \star} \\
(0.0286)\end{array}$ & $\begin{array}{l}-0.138^{* * *} \\
(0.0109)\end{array}$ & $\begin{array}{l}-0.141^{* * *} \\
(0.0131)\end{array}$ & $\begin{array}{l}0.0477^{* * *} \\
(0.00885)\end{array}$ & $\begin{array}{c}-0.00142 \\
(0.0192)\end{array}$ \\
\hline Age70_74 & $\begin{array}{l}-0.0236 \\
(0.0823)\end{array}$ & $\begin{array}{c}0.0279 \\
(0.0637)\end{array}$ & $\begin{array}{l}0.397^{* * *} \\
(0.0325)\end{array}$ & $\begin{array}{l}0.383^{* * *} \\
(0.0508)\end{array}$ & $\begin{array}{c}-0.189^{* * *} \\
(0.0148)\end{array}$ & $\begin{array}{c}-0.191^{* * *} \\
(0.0130)\end{array}$ & $\begin{array}{l}0.0232^{* *} \\
(0.0114)\end{array}$ & $\begin{array}{l}-0.0228 \\
(0.0205)\end{array}$ \\
\hline Age75_79 & $\begin{array}{l}-0.122 \\
(0.100)\end{array}$ & $\begin{array}{c}0.104 \\
(0.138)\end{array}$ & $\begin{array}{l}0.625^{\star \star *} \\
(0.0320)\end{array}$ & $\begin{array}{l}0.610^{* * *} \\
(0.0609)\end{array}$ & $\begin{array}{l}-0.222^{* * *} \\
(0.0175)\end{array}$ & $\begin{array}{c}-0.228^{* * *} \\
(0.0140)\end{array}$ & $\begin{array}{l}-0.00255 \\
(0.0106)\end{array}$ & $\begin{array}{c}-0.0492 \\
(0.0319)\end{array}$ \\
\hline $\begin{array}{l}\text { Age } \\
\text { Above_ } 80\end{array}$ & $\begin{array}{c}-0.302^{\star * *} \\
(0.114)\end{array}$ & $\begin{array}{c}-0.142 \\
(0.0888)\end{array}$ & $\begin{array}{l}0.960^{\star * *} \\
(0.0404)\end{array}$ & $\begin{array}{l}1.053^{\star * \star} \\
(0.0692)\end{array}$ & $\begin{array}{l}-0.255^{\star \star \star} \\
(0.0192)\end{array}$ & $\begin{array}{l}-0.262^{* * *} \\
(0.0144)\end{array}$ & $\begin{array}{c}-0.0836^{* * *} \\
(0.0119)\end{array}$ & $\begin{array}{l}-0.162^{* * *} \\
(0.0286)\end{array}$ \\
\hline Married & $\begin{array}{l}0.184^{* \star} \\
(0.0807)\end{array}$ & $\begin{array}{l}0.482^{* *} \\
(0.160)\end{array}$ & $\begin{array}{l}-0.110^{\star \star *} \\
(0.0395)\end{array}$ & $\begin{array}{c}-0.289^{* * *} \\
(0.0435)\end{array}$ & $\begin{array}{l}-0.0605^{\star * *} \\
(0.00688)\end{array}$ & $\begin{array}{c}-0.0788^{* * *} \\
(0.0119)\end{array}$ & $\begin{array}{c}0.0556^{* * *} \\
(0.0106)\end{array}$ & $\begin{array}{c}0.0167 \\
(0.0194)\end{array}$ \\
\hline $\begin{array}{l}\text { Reg_ } \\
\text { Partnership }\end{array}$ & $\begin{array}{l}0.236^{*} \\
(0.130)\end{array}$ & $\begin{array}{l}0.500^{* *} \\
(0.187)\end{array}$ & $\begin{array}{l}-0.0433 \\
(0.0609)\end{array}$ & $\begin{array}{c}-0.304^{* * *} \\
(0.0623)\end{array}$ & $\begin{array}{r}-0.00607 \\
(0.0189)\end{array}$ & $\begin{array}{l}-0.0500 \\
(0.0391)\end{array}$ & $\begin{array}{l}0.0302^{*} \\
(0.0174)\end{array}$ & $\begin{array}{c}0.0106 \\
(0.0172)\end{array}$ \\
\hline Separated & $\begin{array}{c}0.127 \\
(0.0821)\end{array}$ & $\begin{array}{c}0.103 \\
(0.237)\end{array}$ & $\begin{array}{c}-0.0939^{\star *} \\
(0.0427)\end{array}$ & $\begin{array}{c}-0.472^{* * *} \\
(0.105)\end{array}$ & $\begin{array}{l}0.0312^{*} \\
(0.0168)\end{array}$ & $\begin{array}{c}0.0594 \\
(0.0461)\end{array}$ & $\begin{array}{c}0.0137 \\
(0.0161)\end{array}$ & $\begin{array}{c}0.0548 \\
(0.0450)\end{array}$ \\
\hline Divorced & $\begin{array}{l}0.170^{* * *} \\
(0.0514)\end{array}$ & $\begin{array}{l}0.270^{*} \\
(0.142)\end{array}$ & $\begin{array}{c}-0.0771 \\
(0.0469)\end{array}$ & $\begin{array}{l}-0.221^{\text {** }} \\
(0.0930)\end{array}$ & $\begin{array}{c}0.0494^{\star \star *} \\
(0.0129)\end{array}$ & $\begin{array}{c}0.0273 \\
(0.0406)\end{array}$ & $\begin{array}{c}0.0188^{\star \star} \\
(0.00870)\end{array}$ & $\begin{array}{c}-0.00529 \\
(0.0177)\end{array}$ \\
\hline Widowed & $\begin{array}{c}0.0442 \\
(0.0479)\end{array}$ & $\begin{array}{c}0.155 \\
(0.139)\end{array}$ & $\begin{array}{l}-0.0423 \\
(0.0338)\end{array}$ & $\begin{array}{c}-0.284^{* * *} \\
(0.0728)\end{array}$ & $\begin{array}{l}-0.0206^{* *} \\
(0.00819)\end{array}$ & $\begin{array}{l}-0.0371 \\
(0.0210)\end{array}$ & $\begin{array}{c}0.0646^{\star * \star} \\
(0.0126)\end{array}$ & $\begin{array}{c}0.0194 \\
(0.0214)\end{array}$ \\
\hline Retired & $\begin{array}{l}0.263^{* * *} \\
(0.0573)\end{array}$ & $\begin{array}{l}0.299^{\star \star} \\
(0.111)\end{array}$ & $\begin{array}{l}-0.363^{* * *} \\
(0.0408)\end{array}$ & $\begin{array}{c}-0.380^{* * *} \\
(0.0446)\end{array}$ & $\begin{array}{l}-0.0457^{* * *} \\
(0.00721)\end{array}$ & $\begin{array}{c}-0.0545^{\star *} \\
(0.0233)\end{array}$ & $\begin{array}{c}0.00813 \\
(0.00868)\end{array}$ & $\begin{array}{r}-0.00322 \\
(0.0158)\end{array}$ \\
\hline Employed & $\begin{array}{l}0.281^{* * *} \\
(0.0411)\end{array}$ & $\begin{array}{l}0.326^{\star * *} \\
(0.0923)\end{array}$ & $\begin{array}{l}-0.662^{\star \star *} \\
(0.0310)\end{array}$ & $\begin{array}{c}-0.615^{* * *} \\
(0.0618)\end{array}$ & $\begin{array}{c}-0.0538^{* * *} \\
(0.00938)\end{array}$ & $\begin{array}{c}-0.0741^{* * *} \\
(0.0196)\end{array}$ & $\begin{array}{l}-0.00935 \\
(0.00806)\end{array}$ & $\begin{array}{l}-0.0371^{*} \\
(0.0182)\end{array}$ \\
\hline Homemaker & $\begin{array}{l}-0.0410 \\
(0.0594)\end{array}$ & $\begin{array}{l}-0.179 \\
(0.166)\end{array}$ & $\begin{array}{l}-0.396^{* \star *} \\
(0.0450)\end{array}$ & $\begin{array}{c}-0.464^{* * *} \\
(0.0753)\end{array}$ & $\begin{array}{l}-0.0615^{\star \star *} \\
(0.00864)\end{array}$ & $\begin{array}{c}-0.0974^{* * *} \\
(0.0280)\end{array}$ & $\begin{array}{l}-0.0143 \\
(0.0110)\end{array}$ & $\begin{array}{l}-0.0252 \\
(0.0165)\end{array}$ \\
\hline Other_Job & $\begin{array}{l}0.197^{*} \\
(0.112)\end{array}$ & $\begin{array}{l}0.262^{* *} \\
(0.104)\end{array}$ & $\begin{array}{l}-0.444^{* * *} \\
(0.0530)\end{array}$ & $\begin{array}{c}-0.436^{* * *} \\
(0.0778)\end{array}$ & $\begin{array}{c}-0.0351^{* *} \\
(0.0152)\end{array}$ & $\begin{array}{l}-0.0412 \\
(0.0490)\end{array}$ & $\begin{array}{l}0.00128 \\
(0.0158)\end{array}$ & $\begin{array}{l}-0.0378 \\
(0.0330)\end{array}$ \\
\hline N_Children & $\begin{array}{l}-0.0308^{*} \\
(0.0166)\end{array}$ & $\begin{array}{c}-0.0355^{\star *} \\
(0.0146)\end{array}$ & $\begin{array}{l}-0.0159^{* *} \\
(0.00651)\end{array}$ & $\begin{array}{c}0.00109 \\
(0.00652)\end{array}$ & $\begin{array}{l}-0.00281 \\
(0.00192)\end{array}$ & $\begin{array}{l}-0.00325 \\
(0.00205)\end{array}$ & $\begin{array}{c}0.00403^{* * *} \\
(0.00141)\end{array}$ & $\begin{array}{c}0.00482 \\
(0.00453)\end{array}$ \\
\hline $\begin{array}{l}\text { N_Grand } \\
\text { children }\end{array}$ & $\begin{array}{c}0.00549 \\
(0.00401)\end{array}$ & $\begin{array}{c}0.00588 \\
(0.00716)\end{array}$ & $\begin{array}{l}0.00737^{* *} \\
(0.00363)\end{array}$ & $\begin{array}{c}0.00616 \\
(0.00704)\end{array}$ & $\begin{array}{l}0.00173^{* *} \\
(0.000844)\end{array}$ & $\begin{array}{c}0.00165^{\star} \\
(0.000891)\end{array}$ & $\begin{array}{c}0.00652^{* * *} \\
(0.00125) \\
-\end{array}$ & $\begin{array}{l}0.00900^{* *} \\
(0.00314)\end{array}$ \\
\hline Logincome & $\begin{array}{l}0.0701^{* * *} \\
(0.00813)\end{array}$ & $\begin{array}{c}0.0412 \\
(0.0295)\end{array}$ & $\begin{array}{l}-0.0165^{*} \\
(0.00981)\end{array}$ & $\begin{array}{l}-0.00803^{*} \\
(0.00426)\end{array}$ & $\begin{array}{l}-0.00206^{*} \\
(0.00111)\end{array}$ & $\begin{array}{l}0.000950 \\
(0.00126)\end{array}$ & $\begin{array}{c}0.00355^{* * *} \\
(0.00106)\end{array}$ & $\begin{array}{c}-0.0128^{* * *} \\
(0.00300)\end{array}$ \\
\hline $\begin{array}{l}\text { Year } \\
\text { Dummies } \\
\text { Country }\end{array}$ & YES & YES & YES & YES & YES & YES & YES & YES \\
\hline Dummies & YES & YES & YES & YES & YES & YES & YES & YES \\
\hline $\begin{array}{l}\text { Obs. } \\
\text { R-Squared } \\
\text { N. Resp. }\end{array}$ & $\begin{array}{r}105,943 \\
71,052\end{array}$ & $\begin{array}{c}85,400 \\
0.179\end{array}$ & $\begin{array}{l}105,931 \\
71,049\end{array}$ & $\begin{array}{c}85,400 \\
0.123\end{array}$ & $\begin{array}{c}105,965 \\
71,068\end{array}$ & $\begin{array}{c}85,400 \\
0.062\end{array}$ & $\begin{array}{r}103,516 \\
69,640\end{array}$ & $\begin{array}{c}85,400 \\
0.052\end{array}$ \\
\hline $\begin{array}{l}\text { Dependent } \\
<1 \text { month= } \\
\text { OnceEvery } \\
=\text { dummy } \mathrm{v} \\
\text { (results are }\end{array}$ & $\begin{array}{l}\text { ariables: } \\
\text { 0_in_ } \\
\text { eorThre } \\
\text { able tak } \\
\text { t substa }\end{array}$ & $\begin{array}{l}\text { king: Alm } \\
\text { hs }=1 \text {, } \\
\text { is }=3 \text {, Har } \\
\text { ue } 1 \text { if Ov } \\
\text { different in }\end{array}$ & $\begin{array}{l}\text { Every_Da } \\
\text { tatallinth } \\
\text { ver_neve } \\
\text { ight (29. } \\
\text { red logit }\end{array}$ & $\begin{array}{l}\text { 5or6days } \\
\text { ireemonth } \\
\text { Smoking } \\
<34.9 \text { ) } 0 \\
\text { t estimate }\end{array}$ & $\begin{array}{r}\mathrm{k}=6 \text {, 3or } \\
\text { Sport: } \\
\text { amy varia } \\
\text { ese (BMl }\end{array}$ & $\begin{array}{l}\text { s_week }=5 \text {, } \\
\text { loreThanOr } \\
\text { king value } \\
\text { ). We use } \\
\text { r reasons }\end{array}$ & $\begin{array}{l}\text { 2_week= } \\
\text { week=1, } \\
\text { smoker. } \\
\text { for all d }\end{array}$ & $\begin{array}{l}\text { 1or2_month=3, } \\
\text { OnceAWeek=2, } \\
\text { rWeight_Obese } \\
\text { ndent variables }\end{array}$ \\
\hline
\end{tabular}


Table 4. The impact of education years on health outcomes and functionalities

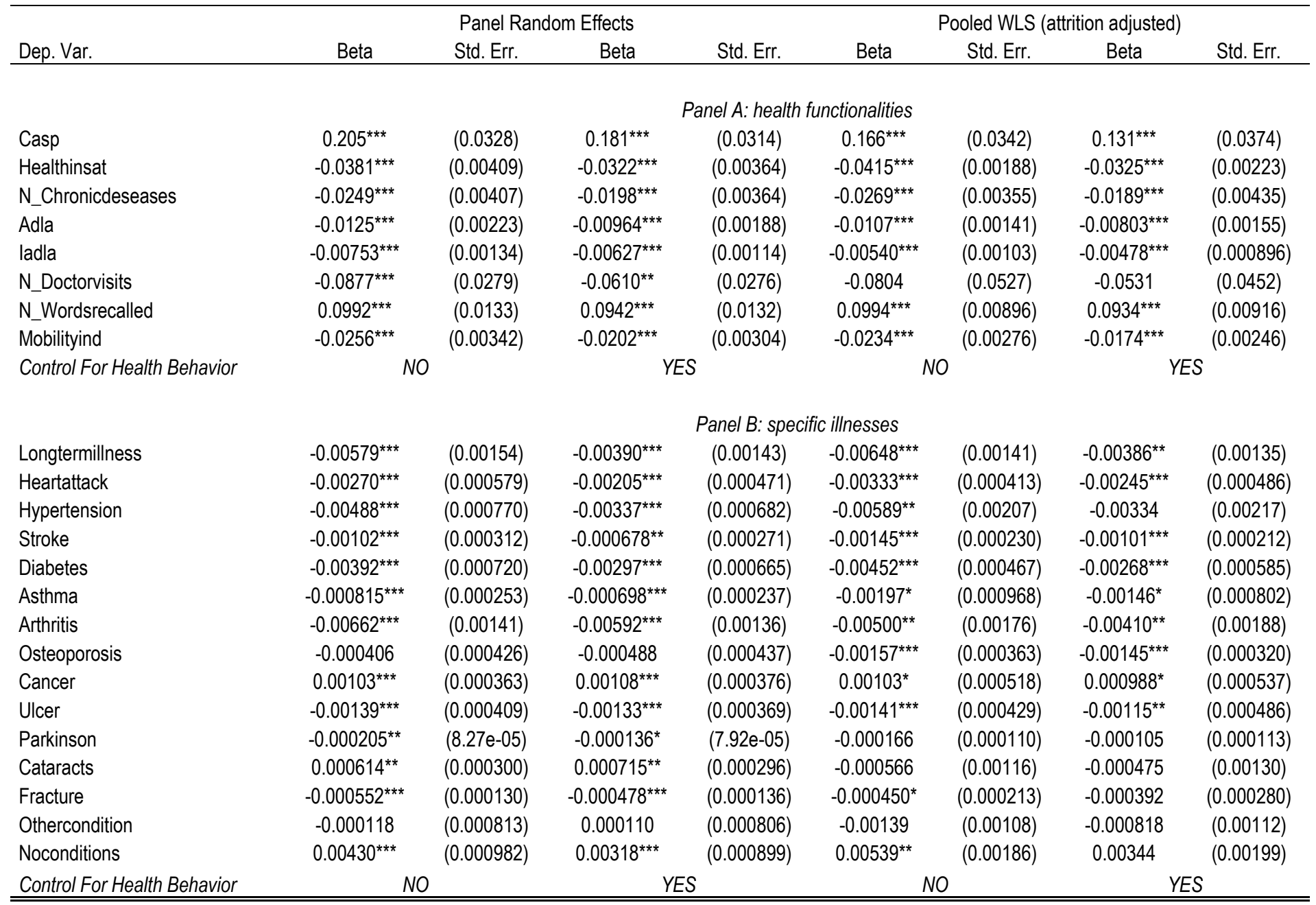

Robust standard errors clustered at country level in parentheses. ${ }^{* *} p<0.01,{ }^{* *} p<0.05,{ }^{*} p<0.1$

Figure 1. Schooling years and quarter of birth (SHARE sample, wave 1)

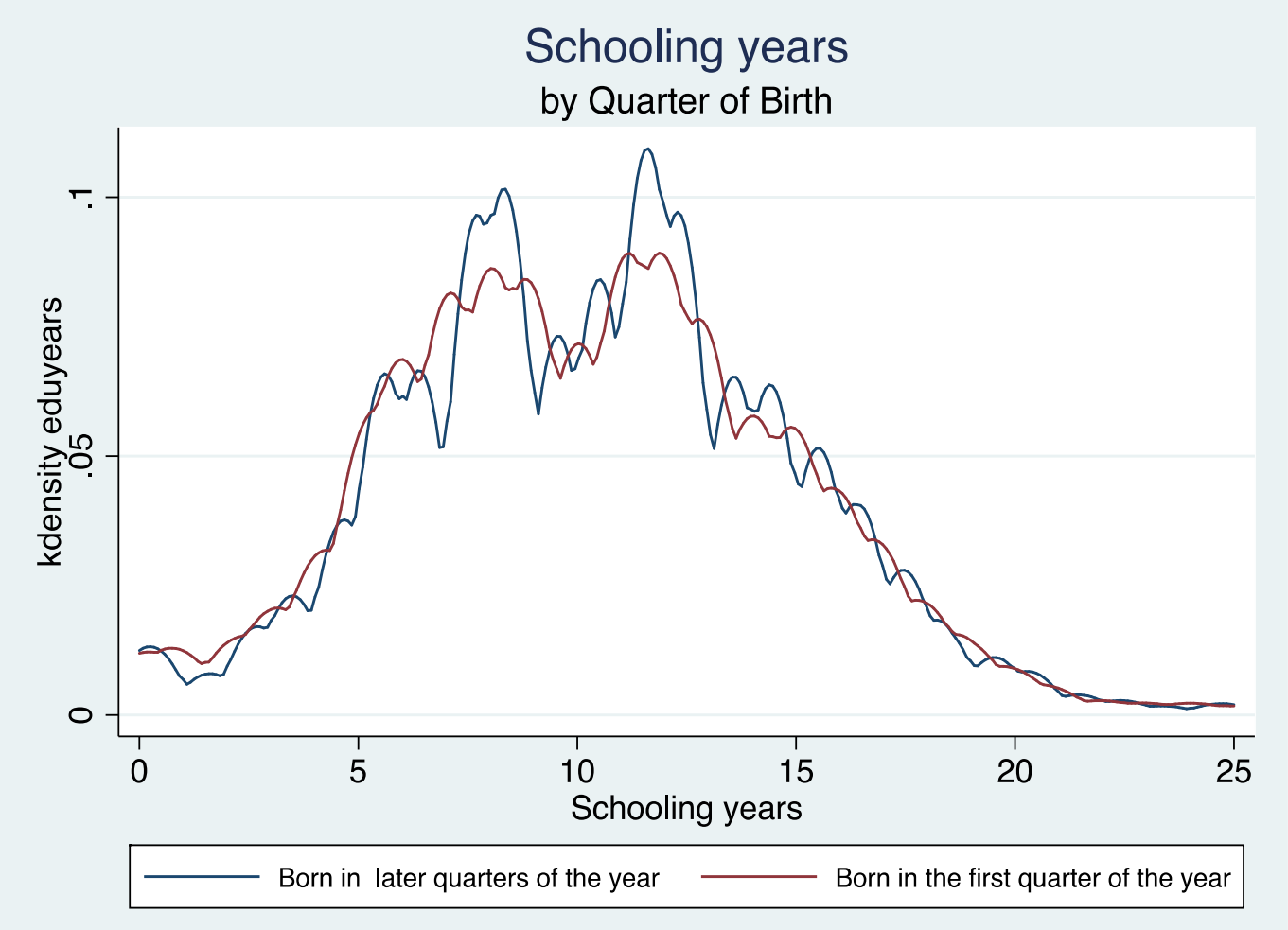


Table 5: The impact of education years on health outcomes and functionalities (IV estimates)

\begin{tabular}{|c|c|c|c|c|c|c|c|c|c|c|c|c|c|c|}
\hline \multirow[b]{4}{*}{ Dep. Var.: } & (1) & (2) & (3) & (4) & (5) & (6) & (7) & (8) & (9) & (10) & (11) & (12) & (13) & (14) \\
\hline & \multicolumn{4}{|c|}{ IV: First_qtr } & \multirow{2}{*}{\multicolumn{4}{|c|}{ IV: $\overline{\text { EduYears }}$}} & & & \multicolumn{3}{|c|}{ IV: First_qtr \& $\overline{\text { EduYears }}$} & \\
\hline & \multicolumn{2}{|c|}{ Education (2nd stage) } & \multicolumn{2}{|c|}{ First_qtr (1st stage) } & \multicolumn{2}{|c|}{ Education (2nd stage) } & & $\overline{\text { EduYears }}$ (1st stage) & \multicolumn{2}{|c|}{ Education (2nd stage) } & \multicolumn{2}{|c|}{ First_qtr (1st stage) } & \multicolumn{2}{|c|}{$\overline{\text { EduYears }}$ (1st stage) } \\
\hline & Beta & Std. Err. & Beta & Std. Err. & Beta & Std. Err. & Beta & Std. Err. & Beta & Std. Err. & Beta & Std. Err. & Beta & Std. Err. \\
\hline \multicolumn{15}{|c|}{ Panel A: health functionalities } \\
\hline Casp & $0.726^{\star * *}$ & $(0.143)$ & $-0.0995^{\star *}$ & $(0.0403)$ & $0.121^{* *}$ & $(0.0580)$ & $0.515^{\star * *}$ & $(0.0825)$ & $0.122^{* *}$ & $(0.0578)$ & $-0.0792^{*}$ & $(0.0414)$ & $0.515^{\star \star *}$ & $(0.0827)$ \\
\hline Healthinsat & $-0.147^{\star \star *}$ & $(0.0262)$ & $-0.0763^{*}$ & $(0.0460)$ & $-0.0314^{\star * *}$ & $(0.00483)$ & $0.515^{\star \star *}$ & $(0.0825)$ & $-0.0314^{\star * *}$ & $(0.00470)$ & $-0.0799^{\star}$ & $(0.0417)$ & $0.515^{\star \star \star}$ & $(0.0827)$ \\
\hline N_Chronicdeseases & $-0.171^{* \star *}$ & $(0.0317)$ & $-0.0762^{*}$ & $(0.0432)$ & -0.00860 & $(0.0111)$ & $0.515^{\star \star *}$ & $(0.0825)$ & -0.00918 & $(0.0112)$ & $-0.0806^{*}$ & $(0.0411)$ & $0.515^{\star \star \star}$ & $(0.0827)$ \\
\hline Adla & $-0.277^{\star * \star}$ & $(0.0251)$ & -0.0310 & $(0.0191)$ & $-0.0143^{\star *}$ & $(0.00717)$ & $0.514^{* * *}$ & $(0.0826)$ & $-0.0143^{\star *}$ & $(0.00703)$ & $-0.0802^{*}$ & $(0.0418)$ & $0.514^{\star \star \star}$ & (0.0828) \\
\hline ladla & $-0.193^{* * *}$ & $(0.0153)$ & $-0.0382^{* * *}$ & $(0.0136)$ & $-0.00985^{\star \star}$ & $(0.00499)$ & $0.514^{\star \star \star}$ & $(0.0827)$ & $-0.0103^{* *}$ & $(0.00489)$ & $-0.0825^{\star \star}$ & $(0.0410)$ & $0.514^{\star \star *}$ & $(0.0828)$ \\
\hline N_Doctorvisits & -0.00137 & $(0.148)$ & $-0.108^{\star \star \star}$ & $(0.0416)$ & 0.0267 & $(0.0852)$ & $0.515^{\star \star \star}$ & $(0.0825)$ & 0.0246 & $(0.0865)$ & $-0.0799^{\star}$ & $(0.0413)$ & $0.515^{\star \star \star}$ & $(0.0827)$ \\
\hline N_Wordsrecalled & $0.524^{* * *}$ & $(0.0618)$ & $-0.0916^{* * *}$ & $(0.0307)$ & $0.0931^{* * *}$ & $(0.0107)$ & $0.515^{\star \star *}$ & $(0.0826)$ & $0.0933^{* * *}$ & $(0.0104)$ & $-0.0802^{* *}$ & $(0.0401)$ & $0.515^{\star * *}$ & $(0.0827)$ \\
\hline Mobilityind & $-0.297^{\star * *}$ & $(0.0319)$ & $-0.0362^{*}$ & $(0.0197)$ & $-0.0205^{\star * *}$ & $(0.00663)$ & $0.515^{\star \star \star}$ & $(0.0826)$ & $-0.0208^{\star \star \star}$ & $(0.00658)$ & $-0.0799^{\star}$ & $(0.0414)$ & $0.515^{\star \star *}$ & (0.0828) \\
\hline \multicolumn{15}{|c|}{ Panel B: Specific Illnesses } \\
\hline Longtermillness & $-0.0351^{\star * *}$ & $(0.00994)$ & $-0.0959^{\star *}$ & $(0.0416)$ & -0.000535 & $(0.00221)$ & $0.515^{\star \star *}$ & $(0.0825)$ & -0.000680 & $(0.00221)$ & $-0.0804^{*}$ & $(0.0416)$ & $0.515^{\star \star \star}$ & $(0.0826)$ \\
\hline Heartattack & $-0.0160^{* *}$ & $(0.00694)$ & $-0.0959^{* *}$ & $(0.0481)$ & -0.000815 & $(0.000816)$ & $0.515^{\star * *}$ & $(0.0825)$ & -0.000899 & $(0.000826)$ & $-0.0806^{*}$ & $(0.0413)$ & $0.515^{\star * *}$ & $(0.0827)$ \\
\hline Hypertension & $-0.0260^{* *}$ & $(0.0101)$ & $-0.0954^{* *}$ & $(0.0444)$ & $-0.00452^{* * *}$ & $(0.00167)$ & $0.515^{\star \star \star}$ & $(0.0825)$ & $-0.00461^{* * *}$ & $(0.00163)$ & $-0.0793^{*}$ & $(0.0414)$ & $0.515^{\star \star *}$ & $(0.0827)$ \\
\hline Stroke & $-0.0160^{\star *}$ & $(0.00726)$ & $-0.0933^{\star *}$ & $(0.0406)$ & -0.000500 & $(0.000705)$ & $0.515^{\star * *}$ & $(0.0825)$ & -0.000676 & $(0.000692)$ & $-0.0799^{*}$ & $(0.0416)$ & $0.515^{\star \star *}$ & $(0.0827)$ \\
\hline Diabetes & 0.00582 & $(0.00561)$ & $-0.107^{\star *}$ & $(0.0418)$ & -0.000826 & $(0.00286)$ & $0.515^{\star * *}$ & $(0.0825)$ & -0.000814 & $(0.00286)$ & $-0.0801^{*}$ & $(0.0413)$ & $0.515^{\star * *}$ & $(0.0827)$ \\
\hline Asthma & 0.00643 & $(0.00512)$ & $-0.0921^{* *}$ & $(0.0413)$ & -0.000851 & $(0.000558)$ & $0.515^{\star \star *}$ & $(0.0825)$ & -0.000975 & $(0.000599)$ & $-0.0805^{\star}$ & $(0.0417)$ & $0.515^{\star \star \star}$ & $(0.0827)$ \\
\hline Arthritis & $-0.0412^{* * *}$ & $(0.00817)$ & $-0.0945^{* *}$ & $(0.0388)$ & -0.00188 & $(0.00216)$ & $0.515^{\star * *}$ & $(0.0825)$ & -0.00206 & $(0.00219)$ & $-0.0800^{*}$ & $(0.0416)$ & $0.515^{\star \star *}$ & $(0.0826)$ \\
\hline Cancer & $-0.00535^{\star}$ & $(0.00316)$ & $-0.0998^{\star *}$ & $(0.0433)$ & $0.00262^{* \star *}$ & $(0.000931)$ & $0.515^{\star \star \star}$ & $(0.0825)$ & $0.00264^{* * *}$ & $(0.000918)$ & $-0.0814^{*}$ & $(0.0417)$ & $0.515^{\star \star *}$ & $(0.0826)$ \\
\hline Ulcer & $9.75 \mathrm{e}-05$ & $(0.00405)$ & $-0.108^{\star * *}$ & $(0.0415)$ & -0.000775 & $(0.000871)$ & $0.515^{\star \star *}$ & $(0.0825)$ & -0.000746 & $(0.000849)$ & $-0.0800^{*}$ & $(0.0414)$ & $0.515^{\star \star *}$ & $(0.0827)$ \\
\hline Parkinsondisease & $-0.0111^{*}$ & $(0.00664)$ & $-0.109^{\star \star *}$ & $(0.0391)$ & -0.000459 & $(0.000423)$ & $0.515^{\star \star \star}$ & $(0.0825)$ & -0.000616 & $(0.000439)$ & $-0.0814^{*}$ & $(0.0416)$ & $0.515^{\star \star \star}$ & $(0.0827)$ \\
\hline Cataracts & $-0.0388^{\star \star *}$ & $(0.00718)$ & $-0.0917^{\star *}$ & $(0.0426)$ & $0.00294^{*}$ & $(0.00174)$ & $0.515^{\star \star *}$ & $(0.0825)$ & $0.00298^{*}$ & $(0.00170)$ & $-0.0786^{*}$ & $(0.0413)$ & $0.515^{\star \star \star}$ & (0.0827) \\
\hline Fracture & $-0.0125^{\star \star *}$ & $(0.00308)$ & $-0.103^{\star * *}$ & $(0.0382)$ & -0.000368 & $(0.000535)$ & $0.515^{\star * *}$ & $(0.0825)$ & -0.000331 & $(0.000520)$ & $-0.0799^{*}$ & $(0.0414)$ & $0.515^{\star * *}$ & $(0.0827)$ \\
\hline Noconditions & $0.0840^{\star * *}$ & $(0.0145)$ & $-0.0675^{\star}$ & $(0.0376)$ & 0.000208 & $(0.00185)$ & $0.515^{\star \star *}$ & $(0.0825)$ & 0.000244 & $(0.00185)$ & $-0.0800^{*}$ & $(0.0414)$ & $0.515^{\star \star \star}$ & $(0.0826)$ \\
\hline
\end{tabular}




\section{APPENDIX}

Table A1 - Descriptive Statistics (health indicators)

\begin{tabular}{|c|c|c|c|c|c|c|c|}
\hline Variable & Obs & Mean & Std. Dev. & Min & Max & \multicolumn{2}{|c|}{$95 \%$ Confidence Intervals } \\
\hline & \multicolumn{7}{|c|}{ Synthetic indicators and functionality indexes } \\
\hline Health_Insat. & 125369 & 3.132 & 1.095 & 1 & 5 & 3.126 & 3.139 \\
\hline N_Doctorvisits & 124269 & 6.780 & 9.887 & 0 & 98 & 6.726 & 6.836 \\
\hline N_Chronicdeseases & 125314 & 1.358 & 1.371 & 0 & 10 & 1.351 & 1.366 \\
\hline Adla & 125327 & 0.218 & 0.749 & 0 & 5 & 0.214 & 0.222 \\
\hline ladla & 125327 & 0.087 & 0.410 & 0 & 3 & 0.085 & 0.089 \\
\hline Mobilityind & 125299 & 0.544 & 0.947 & 0 & 4 & 0.539 & 0.550 \\
\hline N_Wordsrecalled & 122959 & 5.013 & 1.865 & 0 & 10 & 5.003 & 5.024 \\
\hline Numeracy & 104409 & 3.324 & 1.155 & 1 & 5 & 3.317 & 3.331 \\
\hline Casp & 107553 & 37.030 & 6.347 & 12 & 48 & 36.992 & 37.068 \\
\hline \multicolumn{8}{|c|}{ Specific illnesses } \\
\hline Longtermillness & 125409 & 0.506 & 0.500 & 0 & 1 & 0.501 & 0.507 \\
\hline Heartattack & 125314 & 0.131 & 0.337 & 0 & 1 & 0.129 & 0.132 \\
\hline Hypertension & 125314 & 0.362 & 0.481 & 0 & 1 & 0.360 & 0.365 \\
\hline Stroke & 125314 & 0.116 & 0.320 & 0 & 1 & 0.114 & 0.117 \\
\hline Diabetes & 125314 & 0.029 & 0.169 & 0 & 1 & 0.029 & 0.030 \\
\hline Asthma & 125314 & 0.219 & 0.413 & 0 & 1 & 0.217 & 0.221 \\
\hline Arthritis & 125314 & 0.049 & 0.216 & 0 & 1 & 0.048 & 0.050 \\
\hline Osteoporosis & 125314 & 0.049 & 0.216 & 0 & 1 & 0.048 & 0.050 \\
\hline Cancer & 125314 & 0.055 & 0.228 & 0 & 1 & 0.054 & 0.056 \\
\hline Ulcer & 125314 & 0.007 & 0.084 & 0 & 1 & 0.007 & 0.008 \\
\hline Parkinson & 125314 & 0.079 & 0.270 & 0 & 1 & 0.078 & 0.081 \\
\hline Cataracts & 125314 & 0.022 & 0.146 & 0 & 1 & 0.021 & 0.023 \\
\hline Hiporfemoralfracture & 125314 & 0.155 & 0.362 & 0 & 1 & 0.153 & 0.157 \\
\hline Noconditions & 125314 & 0.248 & 0.432 & 0 & 1 & 0.245 & 0.250 \\
\hline
\end{tabular}


Table A2 - Descriptive Statistics (socio-demographic and life style variables)

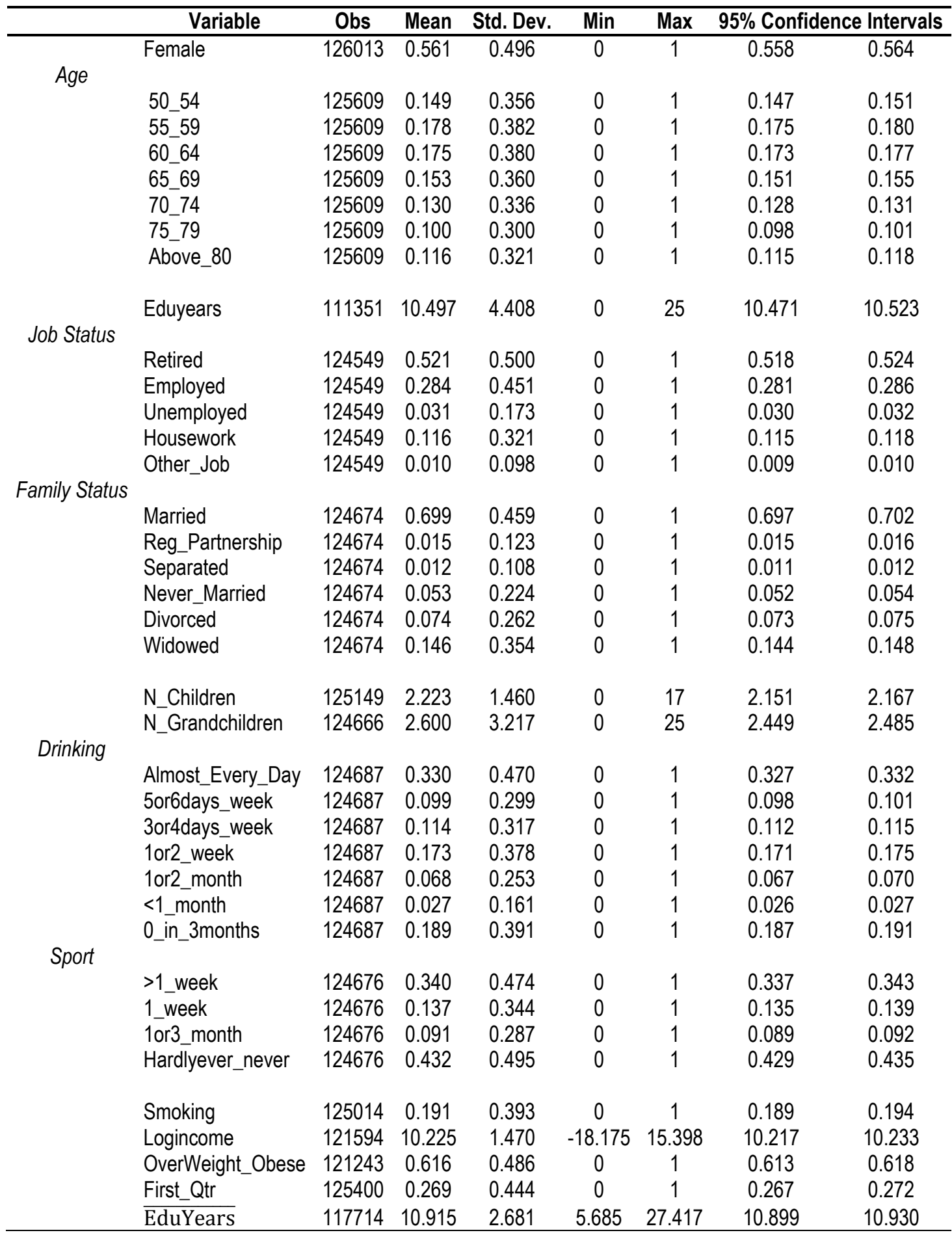


Table A3a - Education years and health functionalities

\begin{tabular}{|c|c|c|c|c|c|c|c|c|}
\hline VARIABLES & $\begin{array}{c}(1) \\
\text { Casp } \\
\end{array}$ & $\begin{array}{c}\text { (2) } \\
\text { health_satisfaction } \\
\end{array}$ & $\begin{array}{c}(3) \\
\text { n_chronicdeseases } \\
\end{array}$ & $\begin{array}{l}(4) \\
\text { adla }\end{array}$ & $\begin{array}{c}(5) \\
\text { iadla }\end{array}$ & $\begin{array}{c}(6) \\
\text { n_doctorvisits } \\
\end{array}$ & $\begin{array}{c}(7) \\
\text { n_wordsrecalled }\end{array}$ & $\begin{array}{c}(8) \\
\text { mobilityind } \\
\end{array}$ \\
\hline Eduyears & $\begin{array}{l}0.205^{* * *} \\
(0.0328)\end{array}$ & $\begin{array}{l}-0.0381^{* * *} \\
(0.00409)\end{array}$ & $\begin{array}{l}-0.0249^{* * *} \\
(0.00407)\end{array}$ & $\begin{array}{l}-0.0125^{* * *} \\
(0.00223)\end{array}$ & $\begin{array}{c}-0.00753^{* * *} \\
(0.00134)\end{array}$ & $\begin{array}{c}-0.0877^{* * *} \\
(0.0279)\end{array}$ & $\begin{array}{l}0.0992^{* * *} \\
(0.0133)\end{array}$ & $\begin{array}{l}-0.0256^{* * *} \\
(0.00342)\end{array}$ \\
\hline Female & $\begin{array}{l}-0.331^{* * *} \\
(0.0875)\end{array}$ & $\begin{array}{l}0.0359^{*} \\
(0.0188)\end{array}$ & $\begin{array}{l}0.0453^{*} \\
(0.0260)\end{array}$ & $\begin{array}{l}0.0215^{\star *} \\
(0.0110)\end{array}$ & $\begin{array}{l}-0.00214 \\
(0.00515)\end{array}$ & $\begin{array}{l}0.653^{\star * *} \\
(0.0988)\end{array}$ & $\begin{array}{l}0.354^{* * *} \\
(0.0337)\end{array}$ & $\begin{array}{l}0.131^{* * *} \\
(0.0170)\end{array}$ \\
\hline Age55_59 & $\begin{array}{c}0.0610 \\
(0.0829)\end{array}$ & $\begin{array}{l}0.108^{\star * \star} \\
(0.0184)\end{array}$ & $\begin{array}{l}0.192^{\star \star \star} \\
(0.0191)\end{array}$ & $\begin{array}{l}-0.00280 \\
(0.00566)\end{array}$ & $\begin{array}{c}-0.00579^{* *} \\
(0.00247)\end{array}$ & $\begin{array}{l}0.217^{*} \\
(0.125)\end{array}$ & $\begin{array}{c}-0.0885^{\star * *} \\
(0.0254)\end{array}$ & $\begin{array}{l}0.0385^{\star \star *} \\
(0.00898)\end{array}$ \\
\hline Age60_64 & $\begin{array}{l}0.222^{*} \\
(0.131)\end{array}$ & $\begin{array}{l}0.111^{* * *} \\
(0.0282)\end{array}$ & $\begin{array}{l}0.347^{\star \star \star} \\
(0.0212)\end{array}$ & $\begin{array}{c}0.0139 \\
(0.00951)\end{array}$ & $\begin{array}{l}-0.00340 \\
(0.00379)\end{array}$ & $\begin{array}{l}0.263 \\
(0.176)\end{array}$ & $\begin{array}{l}-0.194^{* * *} \\
(0.0300)\end{array}$ & $\begin{array}{l}0.0670^{* * *} \\
(0.0171)\end{array}$ \\
\hline Age65_69 & $\begin{array}{l}0.0307 \\
(0.182)\end{array}$ & $\begin{array}{l}0.174^{* * *} \\
(0.0385)\end{array}$ & $\begin{array}{l}0.522^{\star \star *} \\
(0.0325)\end{array}$ & $\begin{array}{l}0.0443^{\star * \star} \\
(0.0146)\end{array}$ & $\begin{array}{l}0.0118^{* *} \\
(0.00507)\end{array}$ & $\begin{array}{l}0.823^{\star * *} \\
(0.300)\end{array}$ & $\begin{array}{l}-0.382^{\star \star *} \\
(0.0376)\end{array}$ & $\begin{array}{l}0.145^{\star \star \star} \\
(0.0283)\end{array}$ \\
\hline Age70_74 & $\begin{array}{c}-0.535^{\star \star *} \\
(0.201)\end{array}$ & $\begin{array}{l}0.310^{\star \star *} \\
(0.0390)\end{array}$ & $\begin{array}{l}0.727^{\star \star \star} \\
(0.0302)\end{array}$ & $\begin{array}{l}0.0981^{\star * \star} \\
(0.0164)\end{array}$ & $\begin{array}{l}0.0323^{\star * \star} \\
(0.00562)\end{array}$ & $\begin{array}{l}1.460^{* \star *} \\
(0.272)\end{array}$ & $\begin{array}{l}-0.721^{* \star *} \\
(0.0396)\end{array}$ & $\begin{array}{l}0.282^{* \star *} \\
(0.0354)\end{array}$ \\
\hline Age75_79 & $\begin{array}{c}-1.274^{* * *} \\
(0.243)\end{array}$ & $\begin{array}{l}0.448^{* \star \star} \\
(0.0432)\end{array}$ & $\begin{array}{l}0.932^{* \star *} \\
(0.0414)\end{array}$ & $\begin{array}{l}0.212^{* \star *} \\
(0.0282)\end{array}$ & $\begin{array}{l}0.0890^{* \star \star} \\
(0.0128)\end{array}$ & $\begin{array}{l}2.131^{* * *} \\
(0.315)\end{array}$ & $\begin{array}{r}-1.082^{* * *} \\
(0.0413)\end{array}$ & $\begin{array}{l}0.494^{* \star *} \\
(0.0467)\end{array}$ \\
\hline AgeAbove_80 & $\begin{array}{c}-2.567^{\star * *} \\
(0.314)\end{array}$ & $\begin{array}{l}0.616^{\star * *} \\
(0.0444)\end{array}$ & $\begin{array}{l}1.013^{* * *} \\
(0.0441)\end{array}$ & $\begin{array}{l}0.509^{* * \star} \\
(0.0472)\end{array}$ & $\begin{array}{l}0.270^{* * *} \\
(0.0265)\end{array}$ & $\begin{array}{c}2.583^{\star * *} \\
(0.362)\end{array}$ & $\begin{array}{c}-1.730^{* * *} \\
(0.0545)\end{array}$ & $\begin{array}{l}0.873^{* \star *} \\
(0.0536)\end{array}$ \\
\hline Married & $\begin{array}{c}0.874^{* * *} \\
(0.121)\end{array}$ & $\begin{array}{l}-0.0305 \\
(0.0273)\end{array}$ & $\begin{array}{c}0.0447 \\
(0.0334)\end{array}$ & $\begin{array}{l}-0.0270^{* *} \\
(0.0117)\end{array}$ & $\begin{array}{c}-0.0355^{* * *} \\
(0.00733)\end{array}$ & $\begin{array}{l}-0.0700 \\
(0.218)\end{array}$ & $\begin{array}{l}0.216^{\star * *} \\
(0.0308)\end{array}$ & $\begin{array}{c}-0.0791^{* * *} \\
(0.0196)\end{array}$ \\
\hline Reg_Partnership & $\begin{array}{c}0.660^{* * *} \\
(0.234)\end{array}$ & $\begin{array}{l}-0.0483 \\
(0.0387)\end{array}$ & $\begin{array}{c}0.0284 \\
(0.0614)\end{array}$ & $\begin{array}{l}-0.00226 \\
(0.0191)\end{array}$ & $\begin{array}{c}-0.0387^{\star \star \star} \\
(0.00827)\end{array}$ & $\begin{array}{l}0.00405 \\
(0.371)\end{array}$ & $\begin{array}{l}0.188^{\star \star \star} \\
(0.0664)\end{array}$ & $\begin{array}{c}-0.0294 \\
(0.0214)\end{array}$ \\
\hline Separated & $\begin{array}{l}-0.278 \\
(0.218)\end{array}$ & $\begin{array}{l}0.00443 \\
(0.0287)\end{array}$ & $\begin{array}{c}0.135^{\star \star} \\
(0.0532)\end{array}$ & $\begin{array}{c}0.0403 \\
(0.0262)\end{array}$ & $\begin{array}{c}-0.0332^{* * *} \\
(0.0103)\end{array}$ & $\begin{array}{l}0.0122 \\
(0.281)\end{array}$ & $\begin{array}{l}0.167^{\star * *} \\
(0.0609)\end{array}$ & $\begin{array}{l}-0.0430 \\
(0.0336)\end{array}$ \\
\hline Divorced & $\begin{array}{c}-0.249 \\
(0.171)\end{array}$ & $\begin{array}{c}0.0109 \\
(0.0244)\end{array}$ & $\begin{array}{l}0.114^{* * *} \\
(0.0293)\end{array}$ & $\begin{array}{c}-0.000745 \\
(0.0136)\end{array}$ & $\begin{array}{c}-0.0389^{* * *} \\
(0.00749)\end{array}$ & $\begin{array}{c}0.273 \\
(0.167)\end{array}$ & $\begin{array}{l}0.183^{* * *} \\
(0.0416)\end{array}$ & $\begin{array}{l}-0.0235 \\
(0.0175)\end{array}$ \\
\hline Widowed & $\begin{array}{c}0.207 \\
(0.140)\end{array}$ & $\begin{array}{l}0.00485 \\
(0.0265)\end{array}$ & $\begin{array}{l}0.155^{\star * *} \\
(0.0341)\end{array}$ & $\begin{array}{l}0.0268^{*} \\
(0.0150)\end{array}$ & $\begin{array}{l}-0.00420 \\
(0.00803)\end{array}$ & $\begin{array}{l}-0.0167 \\
(0.197)\end{array}$ & $\begin{array}{c}0.0121 \\
(0.0335)\end{array}$ & $\begin{array}{l}0.0294^{* *} \\
(0.0131)\end{array}$ \\
\hline Retired & $\begin{array}{c}2.530^{* * *} \\
(0.223)\end{array}$ & $\begin{array}{c}-0.432^{* * *} \\
(0.0401)\end{array}$ & $\begin{array}{l}-0.253^{* * *} \\
(0.0382)\end{array}$ & $\begin{array}{l}-0.243^{* * *} \\
(0.0418)\end{array}$ & $\begin{array}{c}-0.0970^{* * *} \\
(0.0222)\end{array}$ & $\begin{array}{c}-3.050^{* * *} \\
(0.402)\end{array}$ & $\begin{array}{l}0.218^{\star * \star} \\
(0.0257)\end{array}$ & $\begin{array}{l}-0.346^{\star \star *} \\
(0.0408)\end{array}$ \\
\hline Employed & $\begin{array}{c}3.133^{* * *} \\
(0.292)\end{array}$ & $\begin{array}{c}-0.686^{* * *} \\
(0.0354)\end{array}$ & $\begin{array}{c}-0.498^{* * *} \\
(0.0439)\end{array}$ & $\begin{array}{l}-0.269^{* * *} \\
(0.0345)\end{array}$ & $\begin{array}{c}-0.0915^{\star * *} \\
(0.0173)\end{array}$ & $\begin{array}{c}-4.433^{* * *} \\
(0.406)\end{array}$ & $\begin{array}{l}0.336^{\star * *} \\
(0.0417)\end{array}$ & $\begin{array}{l}-0.436^{* * *} \\
(0.0384)\end{array}$ \\
\hline Homemaker & $\begin{array}{c}2.223^{* * *} \\
(0.263)\end{array}$ & $\begin{array}{l}-0.461^{* * *} \\
(0.0437)\end{array}$ & $\begin{array}{c}-0.329^{* * *} \\
(0.0450)\end{array}$ & $\begin{array}{c}-0.286^{\star * \star} \\
(0.0453)\end{array}$ & $\begin{array}{c}-0.0978^{* * *} \\
(0.0255)\end{array}$ & $\begin{array}{c}-3.157^{* * *} \\
(0.374)\end{array}$ & $\begin{array}{l}0.149^{* * *} \\
(0.0545)\end{array}$ & $\begin{array}{l}-0.343^{* * *} \\
(0.0362)\end{array}$ \\
\hline Other_Job & $\begin{array}{c}2.144^{* * *} \\
(0.265)\end{array}$ & $\begin{array}{c}-0.466^{* * *} \\
(0.0460)\end{array}$ & $\begin{array}{c}-0.299^{\star * *} \\
(0.0534)\end{array}$ & $\begin{array}{c}-0.185^{\star \star *} \\
(0.0348)\end{array}$ & $\begin{array}{c}-0.0509^{* * *} \\
(0.0185)\end{array}$ & $\begin{array}{c}-2.943^{* * *} \\
(0.469)\end{array}$ & $\begin{array}{c}0.139^{\star *} \\
(0.0559)\end{array}$ & $\begin{array}{l}-0.300^{* \star *} \\
(0.0453)\end{array}$ \\
\hline N_Children & $\begin{array}{c}-0.0458 \\
(0.0358)\end{array}$ & $\begin{array}{l}-0.0146^{* *} \\
(0.00676)\end{array}$ & $\begin{array}{c}-0.0109 \\
(0.00688)\end{array}$ & $\begin{array}{c}-0.000388 \\
(0.00280)\end{array}$ & $\begin{array}{c}0.00199 \\
(0.00164)\end{array}$ & $\begin{array}{l}0.00726 \\
(0.0484)\end{array}$ & $\begin{array}{c}-0.0162^{*} \\
(0.00892)\end{array}$ & $\begin{array}{c}0.00644 \\
(0.00458)\end{array}$ \\
\hline N_Grandchildren & $\begin{array}{c}0.0200 \\
(0.0134)\end{array}$ & $\begin{array}{c}0.00755^{* * *} \\
(0.00213)\end{array}$ & $\begin{array}{l}0.0190^{\star * *} \\
(0.00298)\end{array}$ & $\begin{array}{l}0.00477^{* *} \\
(0.00202)\end{array}$ & $\begin{array}{l}0.00282^{*} \\
(0.00154)\end{array}$ & $\begin{array}{l}0.0543^{\star} \\
(0.0308)\end{array}$ & $\begin{array}{c}-0.00484 \\
(0.00488)\end{array}$ & $\begin{array}{c}0.00844^{* * *} \\
(0.00232)\end{array}$ \\
\hline Logincome & $\begin{array}{l}0.300^{* * *} \\
(0.0413)\end{array}$ & $\begin{array}{l}-0.0256^{* * *} \\
(0.00750)\end{array}$ & $\begin{array}{c}0.00544 \\
(0.00768)\end{array}$ & $\begin{array}{c}0.00304 \\
(0.00192)\end{array}$ & $\begin{array}{r}-0.000606 \\
(0.00169)\end{array}$ & $\begin{array}{c}0.0256 \\
(0.0442)\end{array}$ & $\begin{array}{c}0.0522^{* * *} \\
(0.0151)\end{array}$ & $\begin{array}{c}-0.00644 \\
(0.00422)\end{array}$ \\
\hline Year dummies & YES & YES & YES & YES & YES & YES & YES & YES \\
\hline Country dummies & YES & YES & YES & YES & YES & YES & YES & YES \\
\hline Observations & 94,439 & 105,917 & 105,884 & 105,914 & 105,914 & 105,255 & 104,287 & 105,896 \\
\hline N. respondents & 67,667 & 71,037 & 71,007 & 71,025 & 71,025 & 70,574 & 70,008 & 71,014 \\
\hline
\end{tabular}


Table A3b - Education years and health functionalities

\begin{tabular}{|c|c|c|c|c|c|c|c|c|}
\hline VARIABLES & $\begin{array}{c}(1) \\
\text { Casp } \\
\end{array}$ & $\begin{array}{c}(2) \\
\text { health_insat. }\end{array}$ & $\begin{array}{c}(3) \\
\text { n_chronicdeseases } \\
\end{array}$ & $\begin{array}{c}(4) \\
\text { adla }\end{array}$ & $\begin{array}{c}(5) \\
\text { iadla }\end{array}$ & $\begin{array}{c}(6) \\
\text { n_doctorvisits } \\
\end{array}$ & $\begin{array}{c}(7) \\
\text { n_wordsrecalled } \\
\end{array}$ & $\begin{array}{c}(8) \\
\text { mobilityind } \\
\end{array}$ \\
\hline Eduyears & $\begin{array}{l}0.181^{* * *} \\
(0.0314)\end{array}$ & $\begin{array}{l}-0.0322^{\star * *} \\
(0.00364)\end{array}$ & $\begin{array}{l}-0.0198^{* * *} \\
(0.00364)\end{array}$ & $\begin{array}{c}-0.00964^{* * *} \\
(0.00188)\end{array}$ & $\begin{array}{c}-0.00627^{* * *} \\
(0.00114)\end{array}$ & $\begin{array}{l}-0.0610^{* *} \\
(0.0276)\end{array}$ & $\begin{array}{l}0.0942^{* * *} \\
(0.0132)\end{array}$ & $\begin{array}{l}-0.0202^{* * *} \\
(0.00304)\end{array}$ \\
\hline DRINKING & & & & & & & & \\
\hline 5or6days_week & $\begin{array}{c}0.731^{* * *} \\
(0.126)\end{array}$ & $\begin{array}{l}-0.130^{\star * *} \\
(0.0170)\end{array}$ & $\begin{array}{l}-0.0547^{\star *} \\
(0.0225)\end{array}$ & $\begin{array}{l}-0.113^{\star * *} \\
(0.0135)\end{array}$ & $\begin{array}{l}-0.0581^{\star * *} \\
(0.00770)\end{array}$ & $\begin{array}{c}-1.141^{\star \star *} \\
(0.165)\end{array}$ & $\begin{array}{l}0.187^{\star \star \star} \\
(0.0220)\end{array}$ & $\begin{array}{l}-0.145^{\star \star *} \\
(0.0185)\end{array}$ \\
\hline 3or4days_week & $\begin{array}{l}0.912^{* \star *} \\
(0.141)\end{array}$ & $\begin{array}{l}-0.186^{* * *} \\
(0.0126)\end{array}$ & $\begin{array}{l}-0.0652^{* *} \\
(0.0259)\end{array}$ & $\begin{array}{l}-0.110^{\star * *} \\
(0.0123)\end{array}$ & $\begin{array}{l}-0.0596^{* * *} \\
(0.00572)\end{array}$ & $\begin{array}{c}-1.335^{\star * *} \\
(0.169)\end{array}$ & $\begin{array}{l}0.252^{* * *} \\
(0.0326)\end{array}$ & $\begin{array}{l}-0.180^{* * *} \\
(0.0137)\end{array}$ \\
\hline 1or2_week & $\begin{array}{l}1.099^{* * *} \\
(0.134)\end{array}$ & $\begin{array}{l}-0.239^{* \star *} \\
(0.0177)\end{array}$ & $\begin{array}{l}-0.0988^{\star * *} \\
(0.0273)\end{array}$ & $\begin{array}{l}-0.127^{\star * *} \\
(0.0119)\end{array}$ & $\begin{array}{l}-0.0607^{* * *} \\
(0.00647)\end{array}$ & $\begin{array}{c}-1.625^{\star * *} \\
(0.214)\end{array}$ & $\begin{array}{l}0.245^{\star * *} \\
(0.0385)\end{array}$ & $\begin{array}{l}-0.190^{* * *} \\
(0.0139)\end{array}$ \\
\hline 1or2_month & $\begin{array}{l}1.048^{* \star *} \\
(0.133)\end{array}$ & $\begin{array}{l}-0.262^{* \star *} \\
(0.0231)\end{array}$ & $\begin{array}{l}-0.100^{* * *} \\
(0.0278)\end{array}$ & $\begin{array}{l}-0.129^{\star * *} \\
(0.0123)\end{array}$ & $\begin{array}{l}-0.0580^{* * *} \\
(0.00722)\end{array}$ & $\begin{array}{c}-1.625^{\star * *} \\
(0.268)\end{array}$ & $\begin{array}{l}0.285^{\star * *} \\
(0.0510)\end{array}$ & $\begin{array}{l}-0.194^{* * *} \\
(0.0135)\end{array}$ \\
\hline$<1$ _month & $\begin{array}{c}0.810^{* * *} \\
(0.102)\end{array}$ & $\begin{array}{l}-0.281^{* * *} \\
(0.0240)\end{array}$ & $\begin{array}{l}-0.132^{* * *} \\
(0.0375)\end{array}$ & $\begin{array}{l}-0.138^{* * *} \\
(0.0154)\end{array}$ & $\begin{array}{l}-0.0625^{* * *} \\
(0.00723)\end{array}$ & $\begin{array}{c}-1.784^{* * *} \\
(0.341)\end{array}$ & $\begin{array}{l}0.337^{* * *} \\
(0.0740)\end{array}$ & $\begin{array}{l}-0.185^{\star \star *} \\
(0.0215)\end{array}$ \\
\hline 0_in_3months & $\begin{array}{l}1.006^{* * *} \\
(0.0832)\end{array}$ & $\begin{array}{l}-0.221^{* * *} \\
(0.0173)\end{array}$ & $\begin{array}{c}-0.0665^{* * *} \\
(0.0224)\end{array}$ & $\begin{array}{l}-0.137^{* * *} \\
(0.0125)\end{array}$ & $\begin{array}{l}-0.0650^{* * *} \\
(0.00820)\end{array}$ & $\begin{array}{c}-1.934^{* * *} \\
(0.178)\end{array}$ & $\begin{array}{l}0.195^{* * *} \\
(0.0522)\end{array}$ & $\begin{array}{l}-0.204^{* * *} \\
(0.0120)\end{array}$ \\
\hline ORT & & & & & & & & \\
\hline 1_week & $\begin{array}{c}-0.537^{\star * *} \\
(0.109)\end{array}$ & $\begin{array}{c}0.0961^{* * *} \\
(0.0101)\end{array}$ & $\begin{array}{l}0.0446^{* *} \\
(0.0184)\end{array}$ & $\begin{array}{c}0.00306 \\
(0.00490)\end{array}$ & $\begin{array}{l}-0.00106 \\
(0.00206)\end{array}$ & $\begin{array}{c}0.375^{\star \star *} \\
(0.103)\end{array}$ & $\begin{array}{c}-0.0182 \\
(0.0283)\end{array}$ & $\begin{array}{l}0.0337^{\star * *} \\
(0.00936)\end{array}$ \\
\hline 1or3_month & $\begin{array}{c}-0.746^{\star * *} \\
(0.120)\end{array}$ & $\begin{array}{l}0.145^{\star * *} \\
(0.0114)\end{array}$ & $\begin{array}{c}0.0788^{* * *} \\
(0.0200)\end{array}$ & $\begin{array}{c}0.00476 \\
(0.00709)\end{array}$ & $\begin{array}{c}0.00191 \\
(0.00350)\end{array}$ & $\begin{array}{c}0.565^{\star \star *} \\
(0.126)\end{array}$ & $\begin{array}{l}-0.0381 \\
(0.0311)\end{array}$ & $\begin{array}{c}0.0704^{* * *} \\
(0.0159)\end{array}$ \\
\hline Hardlyever_never & $\begin{array}{c}-1.770^{\star * *} \\
(0.176)\end{array}$ & $\begin{array}{l}0.435^{\star * *} \\
(0.0186)\end{array}$ & $\begin{array}{l}0.318^{\star * \star} \\
(0.0407)\end{array}$ & $\begin{array}{l}0.179^{\star * \star} \\
(0.0241)\end{array}$ & $\begin{array}{l}0.0609^{* \star *} \\
(0.00927)\end{array}$ & $\begin{array}{c}2.219^{* * *} \\
(0.214)\end{array}$ & $\begin{array}{l}-0.216^{* * *} \\
(0.0336)\end{array}$ & $\begin{array}{l}0.371^{* * *} \\
(0.0345)\end{array}$ \\
\hline Smoking & $\begin{array}{l}-0.486^{* * *} \\
(0.0989)\end{array}$ & $\begin{array}{c}0.0781^{* * *} \\
(0.0167)\end{array}$ & $\begin{array}{c}-0.0469^{\star * *} \\
(0.0169)\end{array}$ & $\begin{array}{l}-0.0212^{* * *} \\
(0.00584)\end{array}$ & $\begin{array}{c}-0.0145^{\star * *} \\
(0.00251)\end{array}$ & $\begin{array}{c}-0.612^{\star * *} \\
(0.120)\end{array}$ & $\begin{array}{c}-0.0652^{\star \star *} \\
(0.0193)\end{array}$ & $\begin{array}{l}0.0254^{* *} \\
(0.00993)\end{array}$ \\
\hline Overweight_Obese & $\begin{array}{l}-0.211^{* * *} \\
(0.0645)\end{array}$ & $\begin{array}{l}0.143^{* * *} \\
(0.0125)\end{array}$ & $\begin{array}{l}0.307^{\star * *} \\
(0.0142)\end{array}$ & $\begin{array}{c}0.0119 \\
(0.00896)\end{array}$ & $\begin{array}{c}-0.0219^{* * *} \\
(0.00518)\end{array}$ & $\begin{array}{l}0.594^{* * *} \\
(0.0798)\end{array}$ & $\begin{array}{l}-0.0171 \\
(0.0177)\end{array}$ & $\begin{array}{l}0.108^{* * *} \\
(0.0122)\end{array}$ \\
\hline Female & $\begin{array}{c}-0.0493 \\
(0.0923)\end{array}$ & $\begin{array}{l}-0.0241 \\
(0.0226)\end{array}$ & $\begin{array}{l}0.0332^{*} \\
(0.0187)\end{array}$ & $\begin{array}{l}-0.0191 \\
(0.0117)\end{array}$ & $\begin{array}{c}-0.0224^{* * *} \\
(0.00635)\end{array}$ & $\begin{array}{c}0.160 \\
(0.119)\end{array}$ & $\begin{array}{l}0.406^{* * *} \\
(0.0368)\end{array}$ & $\begin{array}{c}0.0769^{\star * *} \\
(0.0182)\end{array}$ \\
\hline Age55_59 & $\begin{array}{c}0.0723 \\
(0.0829)\end{array}$ & $\begin{array}{c}0.0978^{* * *} \\
(0.0167)\end{array}$ & $\begin{array}{l}0.172^{\star \star \star} \\
(0.0198)\end{array}$ & $\begin{array}{l}-0.00588 \\
(0.00589)\end{array}$ & $\begin{array}{c}-0.00576^{* *} \\
(0.00233)\end{array}$ & $\begin{array}{c}0.142 \\
(0.119)\end{array}$ & $\begin{array}{c}-0.0868^{\star * *} \\
(0.0253)\end{array}$ & $\begin{array}{l}0.0284^{* * *} \\
(0.00777)\end{array}$ \\
\hline Age60_64 & $\begin{array}{l}0.267^{* *} \\
(0.124)\end{array}$ & $\begin{array}{c}0.0910^{* * *} \\
(0.0256)\end{array}$ & $\begin{array}{l}0.312^{\star \star \star} \\
(0.0192)\end{array}$ & $\begin{array}{c}0.00174 \\
(0.00907)\end{array}$ & $\begin{array}{l}-0.00503 \\
(0.00389)\end{array}$ & $\begin{array}{l}0.0793 \\
(0.155)\end{array}$ & $\begin{array}{c}-0.188^{* * *} \\
(0.0279)\end{array}$ & $\begin{array}{c}0.0452^{* \star *} \\
(0.0144)\end{array}$ \\
\hline Age65_69 & $\begin{array}{c}0.110 \\
(0.175)\end{array}$ & $\begin{array}{l}0.145^{* * *} \\
(0.0353)\end{array}$ & $\begin{array}{l}0.478^{* * *} \\
(0.0310)\end{array}$ & $\begin{array}{l}0.0235^{*} \\
(0.0135)\end{array}$ & $\begin{array}{c}0.00606 \\
(0.00483)\end{array}$ & $\begin{array}{l}0.537^{\star *} \\
(0.268)\end{array}$ & $\begin{array}{c}-0.370^{* * *} \\
(0.0347)\end{array}$ & $\begin{array}{l}0.114^{\star * \star} \\
(0.0258)\end{array}$ \\
\hline Age70_74 & $\begin{array}{l}-0.326^{*} \\
(0.196)\end{array}$ & $\begin{array}{l}0.246^{* * *} \\
(0.0363)\end{array}$ & $\begin{array}{l}0.659^{\star * \star} \\
(0.0293)\end{array}$ & $\begin{array}{c}0.0545^{\star * *} \\
(0.0131)\end{array}$ & $\begin{array}{l}0.0163^{* * *} \\
(0.00487)\end{array}$ & $\begin{array}{c}0.921^{* * *} \\
(0.237)\end{array}$ & $\begin{array}{l}-0.684^{* * *} \\
(0.0379)\end{array}$ & $\begin{array}{l}0.217^{\star * *} \\
(0.0325)\end{array}$ \\
\hline Age75_79 & $\begin{array}{c}-0.912^{\star * *} \\
(0.234)\end{array}$ & $\begin{array}{l}0.347^{\star \star \star} \\
(0.0417)\end{array}$ & $\begin{array}{l}0.840^{\star * \star} \\
(0.0368)\end{array}$ & $\begin{array}{l}0.142^{* \star *} \\
(0.0248)\end{array}$ & $\begin{array}{c}0.0608^{\star \star *} \\
(0.0106)\end{array}$ & $\begin{array}{c}1.413^{* \star *} \\
(0.287)\end{array}$ & $\begin{array}{c}-1.014^{* * *} \\
(0.0424)\end{array}$ & $\begin{array}{l}0.390^{* * *} \\
(0.0428)\end{array}$ \\
\hline AgeAbove_80 & $\begin{array}{c}-1.977^{* * *} \\
(0.298)\end{array}$ & $\begin{array}{l}0.465^{* * *} \\
(0.0429)\end{array}$ & $\begin{array}{l}0.909^{* * *} \\
(0.0477)\end{array}$ & $\begin{array}{l}0.399^{* * *} \\
(0.0415)\end{array}$ & $\begin{array}{l}0.219^{\star * *} \\
(0.0221)\end{array}$ & $\begin{array}{c}1.490^{* * *} \\
(0.366)\end{array}$ & $\begin{array}{c}-1.616^{* \star *} \\
(0.0514)\end{array}$ & $\begin{array}{l}0.718^{\star * *} \\
(0.0495)\end{array}$ \\
\hline Married & $\begin{array}{c}0.726^{* * *} \\
(0.115)\end{array}$ & $\begin{array}{r}-0.00504 \\
(0.0261)\end{array}$ & $\begin{array}{c}0.0319 \\
(0.0294)\end{array}$ & $\begin{array}{l}-0.0121 \\
(0.0125)\end{array}$ & $\begin{array}{c}-0.0231^{* * *} \\
(0.00644)\end{array}$ & $\begin{array}{c}-0.00532 \\
(0.202)\end{array}$ & $\begin{array}{l}0.183^{* * *} \\
(0.0313)\end{array}$ & $\begin{array}{c}-0.0572^{* * *} \\
(0.0191)\end{array}$ \\
\hline Reg_Partnership & $\begin{array}{l}0.606^{* *} \\
(0.272)\end{array}$ & $\begin{array}{l}-0.0376 \\
(0.0426)\end{array}$ & $\begin{array}{c}0.0206 \\
(0.0669)\end{array}$ & $\begin{array}{c}0.0104 \\
(0.0208)\end{array}$ & $\begin{array}{c}-0.0291^{* * *} \\
(0.00723)\end{array}$ & $\begin{array}{l}0.0655 \\
(0.375)\end{array}$ & $\begin{array}{c}0.169^{\star *} \\
(0.0672)\end{array}$ & $\begin{array}{l}-0.0185 \\
(0.0243)\end{array}$ \\
\hline Separated & $\begin{array}{l}-0.351^{*} \\
(0.211)\end{array}$ & $\begin{array}{c}0.0221 \\
(0.0254)\end{array}$ & $\begin{array}{l}0.126^{* *} \\
(0.0512)\end{array}$ & $\begin{array}{l}0.0590^{* *} \\
(0.0268)\end{array}$ & $\begin{array}{l}-0.0187^{* *} \\
(0.00914)\end{array}$ & $\begin{array}{l}0.0426 \\
(0.262)\end{array}$ & $\begin{array}{c}0.154^{* *} \\
(0.0624)\end{array}$ & $\begin{array}{l}-0.0293 \\
(0.0356)\end{array}$ \\
\hline Divorced & $\begin{array}{l}-0.329^{*} \\
(0.184)\end{array}$ & $\begin{array}{c}0.0291 \\
(0.0279)\end{array}$ & $\begin{array}{l}0.115^{* * *} \\
(0.0301)\end{array}$ & $\begin{array}{c}0.0159 \\
(0.0140)\end{array}$ & $\begin{array}{c}-0.0249^{* * *} \\
(0.00603)\end{array}$ & $\begin{array}{l}0.385^{\star *} \\
(0.165)\end{array}$ & $\begin{array}{l}0.160^{* * *} \\
(0.0401)\end{array}$ & $\begin{array}{r}-0.00194 \\
(0.0169)\end{array}$ \\
\hline Widowed & $\begin{array}{c}0.158 \\
(0.143)\end{array}$ & $\begin{array}{l}0.00625 \\
(0.0256)\end{array}$ & $\begin{array}{l}0.126^{* * *} \\
(0.0319)\end{array}$ & $\begin{array}{l}0.0354^{* *} \\
(0.0152)\end{array}$ & $\begin{array}{c}0.00570 \\
(0.00687)\end{array}$ & $\begin{array}{l}-0.0184 \\
(0.196)\end{array}$ & $\begin{array}{l}0.00506 \\
(0.0353)\end{array}$ & $\begin{array}{l}0.0331^{* *} \\
(0.0145)\end{array}$ \\
\hline Retired & $\begin{array}{c}2.237^{* * *} \\
(0.216)\end{array}$ & $\begin{array}{c}-0.367^{\star * *} \\
(0.0355)\end{array}$ & $\begin{array}{l}-0.222^{\star * *} \\
(0.0355)\end{array}$ & $\begin{array}{l}-0.197^{\star * *} \\
(0.0365)\end{array}$ & $\begin{array}{c}-0.0776^{* * *} \\
(0.0193)\end{array}$ & $\begin{array}{c}-2.778^{* * *} \\
(0.363)\end{array}$ & $\begin{array}{l}0.165^{\star * *} \\
(0.0248)\end{array}$ & $\begin{array}{c}-0.288^{* * *} \\
(0.0373)\end{array}$ \\
\hline Employed & $\begin{array}{c}2.637^{* * *} \\
(0.271)\end{array}$ & $\begin{array}{c}-0.573^{\star * *} \\
(0.0315)\end{array}$ & $\begin{array}{l}-0.436^{\star * *} \\
(0.0368)\end{array}$ & $\begin{array}{l}-0.209^{\star * *} \\
(0.0294)\end{array}$ & $\begin{array}{c}-0.0673^{* * *} \\
(0.0152)\end{array}$ & $\begin{array}{c}-3.921^{* * *} \\
(0.358)\end{array}$ & $\begin{array}{l}0.255^{\star * *} \\
(0.0384)\end{array}$ & $\begin{array}{l}-0.341^{* * *} \\
(0.0319)\end{array}$ \\
\hline Homemaker & $\begin{array}{c}1.956^{* * *} \\
(0.231)\end{array}$ & $\begin{array}{c}-0.402^{* * *} \\
(0.0397)\end{array}$ & $\begin{array}{l}-0.298^{* * *} \\
(0.0422)\end{array}$ & $\begin{array}{l}-0.242^{* * *} \\
(0.0403)\end{array}$ & $\begin{array}{c}-0.0827^{* * *} \\
(0.0239)\end{array}$ & $\begin{array}{c}-2.979^{* * *} \\
(0.348)\end{array}$ & $\begin{array}{c}0.110^{* *} \\
(0.0506)\end{array}$ & $\begin{array}{c}-0.291^{* * *} \\
(0.0349)\end{array}$ \\
\hline Other_Job & $\begin{array}{l}1.801^{* * *} \\
(0.261)\end{array}$ & $\begin{array}{l}-0.396^{* * *} \\
(0.0425)\end{array}$ & $\begin{array}{l}-0.266^{* * *} \\
(0.0506)\end{array}$ & $\begin{array}{l}-0.137^{\star * *} \\
(0.0356)\end{array}$ & $\begin{array}{l}-0.0285 \\
(0.0214)\end{array}$ & $\begin{array}{c}-2.607^{* * *} \\
(0.417)\end{array}$ & $\begin{array}{l}0.0909^{*} \\
(0.0484)\end{array}$ & $\begin{array}{c}-0.234^{* * *} \\
(0.0430)\end{array}$ \\
\hline N_Children & $\begin{array}{l}-0.0557^{*} \\
(0.0336)\end{array}$ & $\begin{array}{l}-0.0146^{* *} \\
(0.00669)\end{array}$ & $\begin{array}{c}-0.0105 \\
(0.00693)\end{array}$ & $\begin{array}{l}-0.000971 \\
(0.00285)\end{array}$ & $\begin{array}{c}0.00189 \\
(0.00156)\end{array}$ & $\begin{array}{r}-0.00990 \\
(0.0518)\end{array}$ & $\begin{array}{c}-0.0151^{*} \\
(0.00910)\end{array}$ & $\begin{array}{c}0.00648 \\
(0.00462)\end{array}$ \\
\hline N_Grandchildren & $\begin{array}{l}0.0278^{* *} \\
(0.0120)\end{array}$ & $\begin{array}{c}0.00586^{* * *} \\
(0.00198)\end{array}$ & $\begin{array}{l}0.0156^{\star * *} \\
(0.00278)\end{array}$ & $\begin{array}{l}0.00369^{* *} \\
(0.00179)\end{array}$ & $\begin{array}{l}0.00248^{\star *} \\
(0.00122)\end{array}$ & $\begin{array}{l}0.0463^{*} \\
(0.0270)\end{array}$ & $\begin{array}{l}-0.00418 \\
(0.00441)\end{array}$ & $\begin{array}{c}0.00692^{* * *} \\
(0.00200)\end{array}$ \\
\hline Logincome & $0.274^{\star \star \star}$ & $-0.0196^{\star * *}$ & 0.00888 & $0.00532^{* * *}$ & 0.000729 & $0.0709^{*}$ & $0.0465^{* \star *}$ & -0.00137 \\
\hline
\end{tabular}




\begin{tabular}{lcccccccc} 
& $(0.0380)$ & $(0.00652)$ & $(0.00730)$ & $(0.00193)$ & $(0.00149)$ & $(0.0425)$ & $(0.0145)$ & $(0.00377)$ \\
Year dummies & YES & YES & YES & YES & YES & YES & YES & YES \\
Country dummies & YES & YES & YES & YES & YES & YES & YES & YES \\
Observations & 92,469 & 103,395 & 103,369 & 103,403 & 103,403 & 102,834 & 101,930 & 103,390 \\
N. Respondents & 66,425 & 69,558 & 69,533 & 69,560 & 69,560 & 69,170 & 68,625 & 69,552 \\
\hline
\end{tabular}

Omitted benchmarks: unemployed for job status, never_married for marital status, Austria for country dummies, age50_54 for age dummies, almost_every_day for drinking habits, $<1 \_$week for sport activities. Robust standard errors in parentheses clustered at country level. ${ }^{* *} p<0.01,{ }^{* *}$ $p<0.05,{ }^{*} p<0.1$. 
Table A3c - Education years and health functionalities (attrition-adjusted)

\begin{tabular}{|c|c|c|c|c|c|c|c|c|}
\hline VARIABLES & $\begin{array}{c}(1) \\
\text { Casp } \\
\end{array}$ & $\begin{array}{c}(2) \\
\text { health_satisfaction }\end{array}$ & $\begin{array}{c}(3) \\
\text { n_chronicdeseases } \\
\end{array}$ & $\begin{array}{l}4) \\
\text { adla }\end{array}$ & $\begin{array}{c}(5) \\
\text { iadla }\end{array}$ & $\begin{array}{c}6) \\
\text { n_doctorvisits } \\
\end{array}$ & $\begin{array}{c}(7) \\
\text { n_wordsrecalled } \\
\end{array}$ & $\begin{array}{c}(8) \\
\text { mobilityind } \\
\end{array}$ \\
\hline Eduyears & $\begin{array}{l}-0.229 \\
(0.134)\end{array}$ & $\begin{array}{l}0.0676^{* *} \\
(0.0298)\end{array}$ & $\begin{array}{c}0.0358 \\
(0.0383)\end{array}$ & $\begin{array}{l}0.0288^{* *} \\
(0.0105)\end{array}$ & $\begin{array}{c}0.00118 \\
(0.00367)\end{array}$ & $\begin{array}{l}0.595^{\star \star *} \\
(0.135)\end{array}$ & $\begin{array}{l}0.440^{\star * *} \\
(0.0610)\end{array}$ & $\begin{array}{l}0.147^{* * *} \\
(0.0226)\end{array}$ \\
\hline Female & $\begin{array}{c}0.127 \\
(0.197)\end{array}$ & $\begin{array}{l}0.0937^{* * *} \\
(0.0165)\end{array}$ & $\begin{array}{l}0.230^{* * *} \\
(0.0174)\end{array}$ & $\begin{array}{c}0.00135 \\
(0.00683)\end{array}$ & $\begin{array}{l}-0.00266 \\
(0.00221)\end{array}$ & $\begin{array}{l}0.644^{* * *} \\
(0.185)\end{array}$ & $\begin{array}{l}0.00114 \\
(0.0524)\end{array}$ & $\begin{array}{l}0.0445^{\star *} \\
(0.0155)\end{array}$ \\
\hline Age55_59 & $\begin{array}{c}0.339 \\
(0.225)\end{array}$ & $\begin{array}{c}0.0659 \\
(0.0408)\end{array}$ & $\begin{array}{l}0.343^{\star \star \star} \\
(0.0386)\end{array}$ & $\begin{array}{l}0.00990 \\
(0.0112)\end{array}$ & $\begin{array}{l}-0.00528 \\
(0.00447)\end{array}$ & $\begin{array}{c}0.327 \\
(0.214)\end{array}$ & $\begin{array}{l}-0.0868 \\
(0.0532)\end{array}$ & $\begin{array}{l}0.0642^{* *} \\
(0.0272)\end{array}$ \\
\hline Age60_64 & $\begin{array}{l}0.0493 \\
(0.264)\end{array}$ & $\begin{array}{c}0.0515 \\
(0.0924)\end{array}$ & $\begin{array}{l}0.480^{* * *} \\
(0.0739)\end{array}$ & $\begin{array}{l}0.00884 \\
(0.0282)\end{array}$ & $\begin{array}{c}0.00457 \\
(0.00697)\end{array}$ & $\begin{array}{l}0.857^{* *} \\
(0.357)\end{array}$ & $\begin{array}{l}-0.289^{* *} \\
(0.109)\end{array}$ & $\begin{array}{l}0.120^{* *} \\
(0.0471)\end{array}$ \\
\hline Age65_69 & $\begin{array}{l}-0.0306 \\
(0.267)\end{array}$ & $\begin{array}{l}0.181 \\
(0.105)\end{array}$ & $\begin{array}{l}0.663^{* \star \star} \\
(0.0563)\end{array}$ & $\begin{array}{c}0.0339 \\
(0.0360)\end{array}$ & $\begin{array}{l}0.0158 \\
(0.00958)\end{array}$ & $\begin{array}{l}1.330^{* *} \\
(0.510)\end{array}$ & $\begin{array}{c}-0.607^{\star \star \star} \\
(0.107)\end{array}$ & $\begin{array}{l}0.231^{* \star *} \\
(0.0560)\end{array}$ \\
\hline Age70_74 & $\begin{array}{l}-0.766^{\star *} \\
(0.353)\end{array}$ & $\begin{array}{l}0.340^{* \star \star} \\
(0.0856)\end{array}$ & $\begin{array}{l}0.837^{* \star \star} \\
(0.0596)\end{array}$ & $\begin{array}{c}0.104^{*} \\
(0.0547)\end{array}$ & $\begin{array}{l}0.0374^{*} \\
(0.0174)\end{array}$ & $\begin{array}{l}2.119^{* * *} \\
(0.529)\end{array}$ & $\begin{array}{l}-0.861^{* \star *} \\
(0.0408)\end{array}$ & $\begin{array}{l}0.421^{* \star *} \\
(0.0807)\end{array}$ \\
\hline Age75_79 & $\begin{array}{c}-2.023^{\star \star \star} \\
(0.348)\end{array}$ & $\begin{array}{l}0.439^{\star \star \star} \\
(0.0924)\end{array}$ & $\begin{array}{l}0.918^{\star \star \star} \\
(0.0569)\end{array}$ & $\begin{array}{l}0.345^{\star \star \star} \\
(0.0702)\end{array}$ & $\begin{array}{l}0.184^{\star \star \star} \\
(0.0262)\end{array}$ & $\begin{array}{l}1.776^{\star \star \star} \\
(0.315)\end{array}$ & $\begin{array}{l}-1.490^{* \star \star} \\
(0.0938)\end{array}$ & $\begin{array}{l}0.748^{\star \star \star} \\
(0.0833)\end{array}$ \\
\hline AgeAbove_80 & $\begin{array}{l}0.871^{* *} \\
(0.332)\end{array}$ & $\begin{array}{l}-0.0592 \\
(0.0487)\end{array}$ & $\begin{array}{c}0.0103 \\
(0.0349)\end{array}$ & $\begin{array}{l}-0.0178 \\
(0.0270)\end{array}$ & $\begin{array}{c}-0.0532^{* * *} \\
(0.0116)\end{array}$ & $\begin{array}{l}-0.648^{* *} \\
(0.257)\end{array}$ & $\begin{array}{l}0.303^{* * *} \\
(0.0842)\end{array}$ & $\begin{array}{l}-0.156^{* * *} \\
(0.0366)\end{array}$ \\
\hline Married & $\begin{array}{l}1.130^{\star *} \\
(0.497)\end{array}$ & $\begin{array}{c}-0.0432 \\
(0.0538)\end{array}$ & $\begin{array}{c}-0.116 \\
(0.0883)\end{array}$ & $\begin{array}{c}0.0108 \\
(0.0332)\end{array}$ & $\begin{array}{c}-0.0565^{\star * *} \\
(0.0131)\end{array}$ & $\begin{array}{l}-0.235 \\
(0.312)\end{array}$ & $\begin{array}{l}0.296^{\star \star \star} \\
(0.0885)\end{array}$ & $\begin{array}{l}-0.115^{\star *} \\
(0.0451)\end{array}$ \\
\hline Reg_Partnership & $\begin{array}{l}-1.183 \\
(0.714)\end{array}$ & $\begin{array}{l}-0.0762 \\
(0.0523)\end{array}$ & $\begin{array}{l}0.276^{* *} \\
(0.100)\end{array}$ & $\begin{array}{c}0.0259 \\
(0.0605)\end{array}$ & $\begin{array}{l}-0.0267 \\
(0.0259)\end{array}$ & $\begin{array}{l}-0.939 \\
(0.625)\end{array}$ & $\begin{array}{l}0.0169 \\
(0.252)\end{array}$ & $\begin{array}{c}-0.131 \\
(0.0882)\end{array}$ \\
\hline Separated & $\begin{array}{l}-0.457 \\
(0.405)\end{array}$ & $\begin{array}{c}0.0660 \\
(0.0654)\end{array}$ & $\begin{array}{l}0.194^{* * *} \\
(0.0420)\end{array}$ & $\begin{array}{c}0.0398 \\
(0.0394)\end{array}$ & $\begin{array}{l}-0.0333^{* * *} \\
(0.00991)\end{array}$ & $\begin{array}{c}0.167 \\
(0.508)\end{array}$ & $\begin{array}{c}0.125 \\
(0.0961)\end{array}$ & $\begin{array}{l}-0.00400 \\
(0.0482)\end{array}$ \\
\hline Divorced & $\begin{array}{c}0.434 \\
(0.415)\end{array}$ & $\begin{array}{l}-0.0546 \\
(0.0453)\end{array}$ & $\begin{array}{c}0.129^{*} \\
(0.0651)\end{array}$ & $\begin{array}{c}0.0281 \\
(0.0236)\end{array}$ & $\begin{array}{r}-0.00951 \\
(0.0142)\end{array}$ & $\begin{array}{l}-0.460 \\
(0.366)\end{array}$ & $\begin{array}{c}-0.00786 \\
(0.105)\end{array}$ & $\begin{array}{l}-0.0785^{*} \\
(0.0402)\end{array}$ \\
\hline Widowed & $\begin{array}{c}2.807^{* * *} \\
(0.464)\end{array}$ & $\begin{array}{c}-0.491^{\star * *} \\
(0.107)\end{array}$ & $\begin{array}{l}-0.230^{* *} \\
(0.100)\end{array}$ & $\begin{array}{l}-0.243^{\star * *} \\
(0.0714)\end{array}$ & $\begin{array}{c}-0.0844^{* *} \\
(0.0340)\end{array}$ & $\begin{array}{c}-2.684^{* * *} \\
(0.535)\end{array}$ & $\begin{array}{l}0.166^{\star \star \star} \\
(0.0468)\end{array}$ & $\begin{array}{c}-0.360^{* * *} \\
(0.103)\end{array}$ \\
\hline Retired & $\begin{array}{c}3.394^{* * *} \\
(0.283)\end{array}$ & $\begin{array}{l}-0.827^{\star * *} \\
(0.0638)\end{array}$ & $\begin{array}{l}-0.518^{* * *} \\
(0.0620)\end{array}$ & $\begin{array}{c}-0.280^{* * *} \\
(0.0537)\end{array}$ & $\begin{array}{c}-0.0808^{* * *} \\
(0.0259)\end{array}$ & $\begin{array}{c}-3.712^{\star \star *} \\
(0.422)\end{array}$ & $\begin{array}{l}0.224^{\star * *} \\
(0.0407)\end{array}$ & $\begin{array}{c}-0.423^{* * *} \\
(0.0864)\end{array}$ \\
\hline Employed & $\begin{array}{c}2.567^{* * *} \\
(0.526)\end{array}$ & $\begin{array}{c}-0.560^{* \star *} \\
(0.101)\end{array}$ & $\begin{array}{c}-0.343^{* * *} \\
(0.0931)\end{array}$ & $\begin{array}{l}-0.276^{\star * *} \\
(0.0659)\end{array}$ & $\begin{array}{c}-0.0855^{\star *} \\
(0.0307)\end{array}$ & $\begin{array}{c}-2.324^{*} \\
(0.775)\end{array}$ & $\begin{array}{c}0.142^{*} \\
(0.0686)\end{array}$ & $\begin{array}{c}-0.346^{\star \star \star} \\
(0.101)\end{array}$ \\
\hline Homemaker & $\begin{array}{c}2.739^{* * *} \\
(0.779)\end{array}$ & $\begin{array}{l}-0.525^{\star * *} \\
(0.0841)\end{array}$ & $\begin{array}{l}-0.193^{\star * *} \\
(0.0640)\end{array}$ & $\begin{array}{l}-0.163^{* * *} \\
(0.0463)\end{array}$ & $\begin{array}{l}-0.0435 \\
(0.0301)\end{array}$ & $\begin{array}{c}-2.515^{\star \star *} \\
(0.528)\end{array}$ & $\begin{array}{l}0.0950 \\
(0.115)\end{array}$ & $\begin{array}{l}-0.259^{* * *} \\
(0.0711)\end{array}$ \\
\hline Other_Job & $\begin{array}{c}-0.0977 \\
(0.0753)\end{array}$ & $\begin{array}{c}0.00634 \\
(0.00620)\end{array}$ & $\begin{array}{c}0.00107 \\
(0.00719)\end{array}$ & $\begin{array}{c}-0.00575 \\
(0.00392)\end{array}$ & $\begin{array}{c}-0.00133 \\
(0.00138)\end{array}$ & $\begin{array}{c}-0.0287 \\
(0.0382)\end{array}$ & $\begin{array}{l}-0.0183 \\
(0.0116)\end{array}$ & $\begin{array}{l}0.0153^{\star * *} \\
(0.00506)\end{array}$ \\
\hline N_Children & $\begin{array}{c}0.0117 \\
(0.0252)\end{array}$ & $\begin{array}{l}-0.00410 \\
(0.00358)\end{array}$ & $\begin{array}{l}0.0135^{\star \star *} \\
(0.00384)\end{array}$ & $\begin{array}{c}0.00585 \\
(0.00429)\end{array}$ & $\begin{array}{l}0.00274^{* *} \\
(0.00124)\end{array}$ & $\begin{array}{l}0.0799^{*} \\
(0.0427)\end{array}$ & $\begin{array}{c}6.40 \mathrm{e}-05 \\
(0.00802)\end{array}$ & $\begin{array}{c}0.00633 \\
(0.00529)\end{array}$ \\
\hline N_Grandchildren & $\begin{array}{c}0.0664 \\
(0.0989)\end{array}$ & $\begin{array}{l}-0.0139 \\
(0.0156)\end{array}$ & $\begin{array}{l}0.0512^{\star *} \\
(0.0197)\end{array}$ & $\begin{array}{l}0.0167^{* *} \\
(0.00767)\end{array}$ & $\begin{array}{l}0.00882^{* *} \\
(0.00296)\end{array}$ & $\begin{array}{l}0.197^{\star * *} \\
(0.0644)\end{array}$ & $\begin{array}{c}0.0395^{\star * *} \\
(0.0119)\end{array}$ & $\begin{array}{c}0.0300 \\
(0.0173)\end{array}$ \\
\hline Logincome & $\begin{array}{l}-0.229 \\
(0.134)\end{array}$ & $\begin{array}{l}0.0676^{\star *} \\
(0.0298)\end{array}$ & $\begin{array}{c}0.0358 \\
(0.0383)\end{array}$ & $\begin{array}{l}0.0288^{* *} \\
(0.0105)\end{array}$ & $\begin{array}{c}0.00118 \\
(0.00367)\end{array}$ & $\begin{array}{c}0.595^{\star \star \star} \\
(0.135)\end{array}$ & $\begin{array}{l}0.440^{* * *} \\
(0.0610)\end{array}$ & $\begin{array}{l}0.147^{* * *} \\
(0.0226)\end{array}$ \\
\hline Year dummies & YES & YES & YES & YES & YES & YES & YES & YES \\
\hline Country dummies & YES & YES & YES & YES & YES & YES & YES & YES \\
\hline Observations & 77,549 & 85,374 & 85,400 & 85,377 & 85,377 & 84,935 & 84,283 & 85,370 \\
\hline R-squared & 0.199 & 0.211 & 0.113 & 0.065 & 0.059 & 0.093 & 0.271 & 0.170 \\
\hline
\end{tabular}

Omitted benchmarks: unemployed for job status, never_married for marital status, Austria for country dummies, age50 54 for age dummies, Robust standard errors in parentheses clustered at country level. ${ }^{\star \star *} p<0.01,{ }^{* *} p<0.05,{ }^{*} p<0.1$. 
Table A3d - Education years and health functionalities (attrition-adjusted)

\begin{tabular}{|c|c|c|c|c|c|c|c|c|}
\hline VARIABLES & $\begin{array}{c}1) \\
\text { Casp }\end{array}$ & $\begin{array}{c}\text { (2) } \\
\text { health_insat. }\end{array}$ & $\begin{array}{c}(3) \\
\text { n_chronicdeseases }\end{array}$ & $\begin{array}{l}(4) \\
\text { adla }\end{array}$ & $\begin{array}{c}(5) \\
\text { iadla }\end{array}$ & $\begin{array}{c}6) \\
\text { n_doctorvisits } \\
\end{array}$ & $\begin{array}{c}(7) \\
\text { n_wordsrecalled }\end{array}$ & $\begin{array}{c}(8) \\
\text { mobilityind }\end{array}$ \\
\hline Eduyears & $\begin{array}{l}0.131^{* * *} \\
(0.0374)\end{array}$ & $\begin{array}{l}-0.0325^{\star * *} \\
(0.00223)\end{array}$ & $\begin{array}{l}-0.0189^{* * *} \\
(0.00435)\end{array}$ & $\begin{array}{l}-0.00803^{* * *} \\
(0.00155)\end{array}$ & $\begin{array}{l}-0.00478^{* * *} \\
(0.000896)\end{array}$ & $\begin{array}{l}-0.0531 \\
(0.0452)\end{array}$ & $\begin{array}{l}0.0934^{* * *} \\
(0.00916)\end{array}$ & $\begin{array}{l}-0.0174^{* * *} \\
(0.00246)\end{array}$ \\
\hline DRINKING & & & & & & & & \\
\hline 5or6days_week & $\begin{array}{c}0.512 \\
(0.319)\end{array}$ & $\begin{array}{c}-0.0998^{* * *} \\
(0.0280)\end{array}$ & $\begin{array}{l}-0.103^{* * *} \\
(0.0277)\end{array}$ & $\begin{array}{l}-0.117^{\star * *} \\
(0.0251)\end{array}$ & $\begin{array}{c}-0.0525^{\star * *} \\
(0.0102)\end{array}$ & $\begin{array}{l}-0.846^{*} \\
(0.402)\end{array}$ & $\begin{array}{l}0.222^{* * *} \\
(0.0457)\end{array}$ & $\begin{array}{l}-0.137^{* \star *} \\
(0.0450)\end{array}$ \\
\hline 3or4days_week & $\begin{array}{l}0.809^{* * *} \\
(0.180)\end{array}$ & $\begin{array}{l}-0.156^{\star \star *} \\
(0.0252)\end{array}$ & $\begin{array}{l}-0.109^{\star \star \star} \\
(0.0245)\end{array}$ & $\begin{array}{l}-0.110^{* \star *} \\
(0.0148)\end{array}$ & $\begin{array}{l}-0.0592^{\star \star \star} \\
(0.00657)\end{array}$ & $\begin{array}{l}-0.940^{*} \\
(0.339)\end{array}$ & $\begin{array}{l}0.292^{\star \star \star} \\
(0.0594)\end{array}$ & $\begin{array}{l}-0.160^{\star \star \star} \\
(0.0240)\end{array}$ \\
\hline 1or2_week & $\begin{array}{l}1.270^{* * *} \\
(0.235)\end{array}$ & $\begin{array}{l}-0.241^{* * *} \\
(0.0208)\end{array}$ & $\begin{array}{l}-0.161^{* * *} \\
(0.0353)\end{array}$ & $\begin{array}{l}-0.118^{\star * *} \\
(0.0140)\end{array}$ & $\begin{array}{l}-0.0537^{* * *} \\
(0.00634)\end{array}$ & $\begin{array}{c}-1.216^{* * *} \\
(0.403)\end{array}$ & $\begin{array}{l}0.272^{\star * *} \\
(0.0730)\end{array}$ & $\begin{array}{l}-0.194^{* * *} \\
(0.0210)\end{array}$ \\
\hline 1or2_month & $\begin{array}{l}1.507^{* * *} \\
(0.278)\end{array}$ & $\begin{array}{l}-0.322^{\star * *} \\
(0.0438)\end{array}$ & $\begin{array}{l}-0.162^{* * *} \\
(0.0423)\end{array}$ & $\begin{array}{l}-0.110^{\star * *} \\
(0.0258)\end{array}$ & $\begin{array}{c}-0.0504^{* * *} \\
(0.0121)\end{array}$ & $\begin{array}{l}-0.701 \\
(0.566)\end{array}$ & $\begin{array}{l}0.339^{* * *} \\
(0.0686)\end{array}$ & $\begin{array}{l}-0.186^{* * *} \\
(0.0272)\end{array}$ \\
\hline$<1$ _month & $\begin{array}{l}1.495^{\star \star *} \\
(0.290)\end{array}$ & $\begin{array}{l}-0.319^{* * *} \\
(0.0522)\end{array}$ & $\begin{array}{l}-0.247^{\star \star *} \\
(0.0555)\end{array}$ & $\begin{array}{l}-0.124^{\star * *} \\
(0.0224)\end{array}$ & $\begin{array}{l}-0.0322^{*} \\
(0.0163)\end{array}$ & $\begin{array}{l}-1.747^{* *} \\
(0.759)\end{array}$ & $\begin{array}{c}0.544^{\star * *} \\
(0.125)\end{array}$ & $\begin{array}{l}-0.185^{\star * *} \\
(0.0504)\end{array}$ \\
\hline 0_in_3months & $\begin{array}{l}1.228^{* * *} \\
(0.163)\end{array}$ & $\begin{array}{l}-0.240^{* * *} \\
(0.0327)\end{array}$ & $\begin{array}{l}-0.114^{* * *} \\
(0.0322)\end{array}$ & $\begin{array}{l}-0.116^{* * *} \\
(0.00925)\end{array}$ & $\begin{array}{l}-0.0489^{* * *} \\
(0.00780)\end{array}$ & $\begin{array}{c}-1.363^{* * *} \\
(0.442)\end{array}$ & $\begin{array}{l}0.339^{* * *} \\
(0.0529)\end{array}$ & $\begin{array}{l}-0.191^{* * *} \\
(0.0181)\end{array}$ \\
\hline SPORT & & & & & & & & \\
\hline 1_week & $\begin{array}{l}-0.265 \\
(0.210)\end{array}$ & $\begin{array}{l}0.112^{* \star *} \\
(0.0320)\end{array}$ & $\begin{array}{c}0.0469 \\
(0.0555)\end{array}$ & $\begin{array}{l}0.000916 \\
(0.00568)\end{array}$ & $\begin{array}{c}-0.000540 \\
(0.00244)\end{array}$ & $\begin{array}{c}0.542^{* * *} \\
(0.143)\end{array}$ & $\begin{array}{l}-0.0248 \\
(0.0323)\end{array}$ & $\begin{array}{c}0.0498^{* * *} \\
(0.0122)\end{array}$ \\
\hline 1or3_month & $\begin{array}{c}-0.631^{* \star \star} \\
(0.180)\end{array}$ & $\begin{array}{l}0.148^{\star \star \star} \\
(0.0180)\end{array}$ & $\begin{array}{c}0.0669^{\star \star \star} \\
(0.0221)\end{array}$ & $\begin{array}{c}-0.0152 \\
(0.00880)\end{array}$ & $\begin{array}{l}-0.00526 \\
(0.00737)\end{array}$ & $\begin{array}{c}0.380 \\
(0.218)\end{array}$ & $\begin{array}{c}0.0169 \\
(0.0460)\end{array}$ & $\begin{array}{l}0.0623^{\star *} \\
(0.0264)\end{array}$ \\
\hline Hardlyever_never & $\begin{array}{c}-1.817^{* * *} \\
(0.142)\end{array}$ & $\begin{array}{l}0.518^{* * *} \\
(0.0407)\end{array}$ & $\begin{array}{l}0.368^{* * *} \\
(0.0319)\end{array}$ & $\begin{array}{l}0.182^{\star * *} \\
(0.0170)\end{array}$ & $\begin{array}{l}0.0623^{* * *} \\
(0.00815)\end{array}$ & $\begin{array}{c}2.222^{* * *} \\
(0.391)\end{array}$ & $\begin{array}{c}-0.246^{\star * *} \\
(0.0610)\end{array}$ & $\begin{array}{l}0.405^{\star * \star} \\
(0.0316)\end{array}$ \\
\hline Smoking & $\begin{array}{c}-0.434^{* * *} \\
(0.108)\end{array}$ & $\begin{array}{c}0.0715^{\star * *} \\
(0.0233)\end{array}$ & $\begin{array}{c}-0.0944^{* * *} \\
(0.0226)\end{array}$ & $\begin{array}{c}-0.0114^{*} \\
(0.00609)\end{array}$ & $\begin{array}{l}-0.0200^{* * *} \\
(0.00395)\end{array}$ & $\begin{array}{c}-0.842^{* * *} \\
(0.256)\end{array}$ & $\begin{array}{l}-0.0831^{* *} \\
(0.0338)\end{array}$ & $\begin{array}{c}0.0132 \\
(0.0150)\end{array}$ \\
\hline Overweight_Obese & $\begin{array}{l}-0.329^{* * *} \\
(0.0792)\end{array}$ & $\begin{array}{c}0.158^{\star * *} \\
(0.00891)\end{array}$ & $\begin{array}{l}0.320^{* * *} \\
(0.0148)\end{array}$ & $\begin{array}{l}0.0195^{\star *} \\
(0.00680)\end{array}$ & $\begin{array}{c}-0.00941^{* *} \\
(0.00397)\end{array}$ & $\begin{array}{l}0.433^{* *} \\
(0.176)\end{array}$ & $\begin{array}{l}0.00494 \\
(0.0319)\end{array}$ & $\begin{array}{l}0.101^{* \star *} \\
(0.0165)\end{array}$ \\
\hline Female & $\begin{array}{l}0.0726 \\
(0.125)\end{array}$ & $\begin{array}{l}0.00128 \\
(0.0296)\end{array}$ & $\begin{array}{c}0.0158 \\
(0.0402)\end{array}$ & $\begin{array}{c}0.000140 \\
(0.0115)\end{array}$ & $\begin{array}{l}-0.0116^{* *} \\
(0.00417)\end{array}$ & $\begin{array}{l}0.226^{*} \\
(0.123)\end{array}$ & $\begin{array}{l}0.507^{* * *} \\
(0.0580)\end{array}$ & $\begin{array}{c}0.0967^{* * *} \\
(0.0237)\end{array}$ \\
\hline Age55_59 & $\begin{array}{c}0.134 \\
(0.187)\end{array}$ & $\begin{array}{c}0.0853^{* * *} \\
(0.0155)\end{array}$ & $\begin{array}{l}0.216^{* * *} \\
(0.0156)\end{array}$ & $\begin{array}{l}-0.00251 \\
(0.00742)\end{array}$ & $\begin{array}{c}-0.00442^{* *} \\
(0.00196)\end{array}$ & $\begin{array}{c}0.576^{* * *} \\
(0.185)\end{array}$ & $\begin{array}{r}-0.00111 \\
(0.0504)\end{array}$ & $\begin{array}{l}0.0354^{* *} \\
(0.0157)\end{array}$ \\
\hline Age60_64 & $\begin{array}{c}0.350 \\
(0.222)\end{array}$ & $\begin{array}{c}0.0547 \\
(0.0377)\end{array}$ & $\begin{array}{l}0.315^{\star \star \star} \\
(0.0348)\end{array}$ & $\begin{array}{l}0.00375 \\
(0.0111)\end{array}$ & $\begin{array}{l}-0.00836 \\
(0.00514)\end{array}$ & $\begin{array}{c}0.191 \\
(0.210)\end{array}$ & $\begin{array}{l}-0.0954^{*} \\
(0.0463)\end{array}$ & $\begin{array}{l}0.0502^{*} \\
(0.0240)\end{array}$ \\
\hline Age65_69 & $\begin{array}{l}0.0949 \\
(0.232)\end{array}$ & $\begin{array}{c}0.0301 \\
(0.0813)\end{array}$ & $\begin{array}{l}0.441^{* \star *} \\
(0.0685)\end{array}$ & $\begin{array}{r}-0.00586 \\
(0.0247)\end{array}$ & $\begin{array}{c}-0.00351 \\
(0.00598)\end{array}$ & $\begin{array}{c}0.599 \\
(0.354)\end{array}$ & $\begin{array}{l}-0.284^{* *} \\
(0.0953)\end{array}$ & $\begin{array}{l}0.0949^{\star *} \\
(0.0382)\end{array}$ \\
\hline Age70_74 & $\begin{array}{c}0.142 \\
(0.234)\end{array}$ & $\begin{array}{c}0.125 \\
(0.0912)\end{array}$ & $\begin{array}{l}0.599^{* * *} \\
(0.0507)\end{array}$ & $\begin{array}{l}0.00341 \\
(0.0336)\end{array}$ & $\begin{array}{c}0.000632 \\
(0.0100)\end{array}$ & $\begin{array}{c}0.871 \\
(0.498)\end{array}$ & $\begin{array}{l}-0.581^{* * *} \\
(0.0922)\end{array}$ & $\begin{array}{l}0.177^{\star * \star} \\
(0.0470)\end{array}$ \\
\hline Age75_79 & $\begin{array}{l}-0.441 \\
(0.293)\end{array}$ & $\begin{array}{l}0.248^{\star * *} \\
(0.0737)\end{array}$ & $\begin{array}{l}0.745^{\star \star *} \\
(0.0546)\end{array}$ & $\begin{array}{c}0.0577 \\
(0.0509)\end{array}$ & $\begin{array}{c}0.0154 \\
(0.0169)\end{array}$ & $\begin{array}{l}1.454^{* *} \\
(0.562)\end{array}$ & $\begin{array}{l}-0.817^{* * *} \\
(0.0353)\end{array}$ & $\begin{array}{l}0.336^{\star \star \star} \\
(0.0669)\end{array}$ \\
\hline AgeAbove_80 & $\begin{array}{c}-1.379^{* * *} \\
(0.370)\end{array}$ & $\begin{array}{l}0.266^{\star * \star} \\
(0.0778)\end{array}$ & $\begin{array}{l}0.788^{* * *} \\
(0.0503)\end{array}$ & $\begin{array}{l}0.260^{* \star *} \\
(0.0645)\end{array}$ & $\begin{array}{l}0.145^{* * *} \\
(0.0250)\end{array}$ & $\begin{array}{c}0.706 \\
(0.408)\end{array}$ & $\begin{array}{c}-1.383^{* * *} \\
(0.0824)\end{array}$ & $\begin{array}{l}0.593^{* * *} \\
(0.0665)\end{array}$ \\
\hline Married & $\begin{array}{l}0.564^{*} \\
(0.292)\end{array}$ & $\begin{array}{c}0.0216 \\
(0.0542)\end{array}$ & $\begin{array}{c}0.0494 \\
(0.0301)\end{array}$ & $\begin{array}{l}0.00938 \\
(0.0293)\end{array}$ & $\begin{array}{c}-0.0446^{* * *} \\
(0.0117)\end{array}$ & $\begin{array}{l}-0.398 \\
(0.234)\end{array}$ & $\begin{array}{c}0.245^{\star *} \\
(0.0837)\end{array}$ & $\begin{array}{c}-0.0981^{\text {** }} \\
(0.0358)\end{array}$ \\
\hline Reg_Partnership & $\begin{array}{l}0.856^{*} \\
(0.461)\end{array}$ & $\begin{array}{c}0.0348 \\
(0.0627)\end{array}$ & $\begin{array}{l}-0.0765 \\
(0.0865)\end{array}$ & $\begin{array}{c}0.0361 \\
(0.0350)\end{array}$ & $\begin{array}{c}-0.0474^{* * *} \\
(0.0137)\end{array}$ & $\begin{array}{l}0.0379 \\
(0.348)\end{array}$ & $\begin{array}{c}0.242^{* *} \\
(0.0924)\end{array}$ & $\begin{array}{l}-0.0580 \\
(0.0476)\end{array}$ \\
\hline Separated & $\begin{array}{l}-1.453^{*} \\
(0.675)\end{array}$ & $\begin{array}{r}-0.00347 \\
(0.0441)\end{array}$ & $\begin{array}{l}0.328^{\star *} \\
(0.109)\end{array}$ & $\begin{array}{c}0.0576 \\
(0.0583)\end{array}$ & $\begin{array}{l}-0.0145 \\
(0.0247)\end{array}$ & $\begin{array}{l}-0.517 \\
(0.645)\end{array}$ & $\begin{array}{l}-0.0196 \\
(0.249)\end{array}$ & $\begin{array}{l}-0.0680 \\
(0.0818)\end{array}$ \\
\hline Divorced & $\begin{array}{l}-0.606 \\
(0.425)\end{array}$ & $\begin{array}{c}0.119 \\
(0.0830)\end{array}$ & $\begin{array}{l}0.237^{* * *} \\
(0.0424)\end{array}$ & $\begin{array}{c}0.0631 \\
(0.0450)\end{array}$ & $\begin{array}{c}-0.0244^{* *} \\
(0.0113)\end{array}$ & $\begin{array}{c}0.433 \\
(0.545)\end{array}$ & $\begin{array}{c}0.0881 \\
(0.0898)\end{array}$ & $\begin{array}{c}0.0406 \\
(0.0604)\end{array}$ \\
\hline Widowed & $\begin{array}{c}0.208 \\
(0.384)\end{array}$ & $\begin{array}{l}0.00866 \\
(0.0440)\end{array}$ & $\begin{array}{c}0.165^{\star \star} \\
(0.0575)\end{array}$ & $\begin{array}{l}0.0523^{*} \\
(0.0260)\end{array}$ & $\begin{array}{r}-0.00104 \\
(0.0151)\end{array}$ & $\begin{array}{l}-0.227 \\
(0.349)\end{array}$ & $\begin{array}{l}-0.0486 \\
(0.102)\end{array}$ & $\begin{array}{l}-0.0289 \\
(0.0409)\end{array}$ \\
\hline Retired & $\begin{array}{c}2.487^{\star * *} \\
(0.443)\end{array}$ & $\begin{array}{l}-0.408^{* \star \star} \\
(0.0937)\end{array}$ & $\begin{array}{l}-0.181^{*} \\
(0.0943)\end{array}$ & $\begin{array}{l}-0.215^{\star \star *} \\
(0.0667)\end{array}$ & $\begin{array}{c}-0.0757^{* *} \\
(0.0316)\end{array}$ & $\begin{array}{c}-2.394^{* * *} \\
(0.511)\end{array}$ & $\begin{array}{c}0.116^{* *} \\
(0.0429)\end{array}$ & $\begin{array}{l}-0.299^{* * *} \\
(0.0920)\end{array}$ \\
\hline Employed & $\begin{array}{c}2.847^{\star * *} \\
(0.304)\end{array}$ & $\begin{array}{l}-0.688^{\star \star \star} \\
(0.0530)\end{array}$ & $\begin{array}{l}-0.426^{* \star \star} \\
(0.0671)\end{array}$ & $\begin{array}{c}-0.235^{\star \star *} \\
(0.0524)\end{array}$ & $\begin{array}{c}-0.0667^{* *} \\
(0.0243)\end{array}$ & $\begin{array}{c}-3.226^{* * *} \\
(0.381)\end{array}$ & $\begin{array}{l}0.149^{* * *} \\
(0.0492)\end{array}$ & $\begin{array}{l}-0.321^{* * *} \\
(0.0819)\end{array}$ \\
\hline Homemaker & $\begin{array}{c}2.254^{* * *} \\
(0.514)\end{array}$ & $\begin{array}{l}-0.474^{* * *} \\
(0.0932)\end{array}$ & $\begin{array}{l}-0.288^{* * *} \\
(0.0947)\end{array}$ & $\begin{array}{l}-0.250^{\star * *} \\
(0.0660)\end{array}$ & $\begin{array}{c}-0.0793^{* *} \\
(0.0297)\end{array}$ & $\begin{array}{l}-2.093^{* *} \\
(0.787)\end{array}$ & $\begin{array}{c}0.107 \\
(0.0685)\end{array}$ & $\begin{array}{l}-0.283^{* *} \\
(0.0976)\end{array}$ \\
\hline Other_Job & $\begin{array}{l}2.322^{* *} \\
(0.775)\end{array}$ & $\begin{array}{l}-0.437^{* * *} \\
(0.0810)\end{array}$ & $\begin{array}{l}-0.134^{*} \\
(0.0730)\end{array}$ & $\begin{array}{l}-0.138^{* *} \\
(0.0459)\end{array}$ & $\begin{array}{l}-0.0363 \\
(0.0277)\end{array}$ & $\begin{array}{c}-2.220^{* * *} \\
(0.537)\end{array}$ & $\begin{array}{l}0.0490 \\
(0.118)\end{array}$ & $\begin{array}{l}-0.196^{* *} \\
(0.0779)\end{array}$ \\
\hline N_Children & $\begin{array}{l}-0.0898 \\
(0.0749)\end{array}$ & $\begin{array}{c}0.00277 \\
(0.00663)\end{array}$ & $\begin{array}{l}-0.00284 \\
(0.00743)\end{array}$ & $\begin{array}{l}-0.00730 \\
(0.00445)\end{array}$ & $\begin{array}{l}-0.00189 \\
(0.00147)\end{array}$ & $\begin{array}{l}-0.0473 \\
(0.0402)\end{array}$ & $\begin{array}{l}-0.0158 \\
(0.0111)\end{array}$ & $\begin{array}{c}0.0124^{* *} \\
(0.00548)\end{array}$ \\
\hline N_Grandchildren & $\begin{array}{c}0.0178 \\
(0.0253)\end{array}$ & $\begin{array}{l}-0.00596 \\
(0.00390)\end{array}$ & $\begin{array}{l}0.0105^{\star *} \\
(0.00370)\end{array}$ & $\begin{array}{c}0.00569 \\
(0.00441)\end{array}$ & $\begin{array}{l}0.00287^{\star *} \\
(0.00115)\end{array}$ & $\begin{array}{l}0.0759^{*} \\
(0.0382)\end{array}$ & $\begin{array}{l}-2.53 e-05 \\
(0.00847)\end{array}$ & $\begin{array}{c}0.00519 \\
(0.00486)\end{array}$ \\
\hline Logincome & 0.0470 & -0.00767 & $0.0584^{* *}$ & $0.0192^{* *}$ & $0.00962^{* * *}$ & $0.226^{* * *}$ & $0.0349^{* * *}$ & $0.0349^{*}$ \\
\hline
\end{tabular}




\begin{tabular}{lccccccccc} 
& $(0.0949)$ & $(0.0141)$ & $(0.0198)$ & $(0.00748)$ & $(0.00294)$ & $(0.0616)$ & $(0.0115)$ & $(0.0167)$ \\
Year dummies & YES & YES & YES & YES & YES & YES & YES & YES \\
Country dummies & YES & YES & YES & YES & YES & YES & YES & YES \\
Observations & 77,549 & 85,374 & 85,400 & 85,377 & 85,377 & 84,935 & 84,283 & 85,370 \\
R-squared & 0.227 & 0.271 & 0.147 & 0.093 & 0.074 & 0.110 & 0.281 & 0.231 \\
\hline
\end{tabular}

Omitted benchmarks: unemployed for job status, never_married for marital status, Austria for country dummies, age50_54 for age dummies, almost_every_day for drinking habits, $<1 \_$week for sport activities. Robust standard errors in parentheses clustered at country level. ${ }^{* * *} p<0.01,{ }^{* *}$ $p<0.05,{ }^{*} p<0.1$. 
Table A4a - Education years and specific diseases

\begin{tabular}{|c|c|c|c|c|c|c|c|c|c|c|c|c|c|c|c|}
\hline VARIABLES & $\begin{array}{c}1) \\
\text { Longterm } \\
\text { illness } \\
\end{array}$ & $\begin{array}{c}(2) \\
\text { heart } \\
\text { attack }\end{array}$ & $\begin{array}{c}\text { (3) } \\
\text { hyper } \\
\text { tension }\end{array}$ & $\begin{array}{c}(4) \\
\text { stroke }\end{array}$ & $\begin{array}{c}\text { (5) } \\
\text { diabetes } \\
\end{array}$ & $\begin{array}{c}(6) \\
\text { asthma }\end{array}$ & $\begin{array}{c}(7) \\
\text { arthritis }\end{array}$ & $\begin{array}{c}\text { (8) } \\
\text { osteopor } \\
\end{array}$ & $\begin{array}{c}\text { (9) } \\
\text { cancer }\end{array}$ & $\begin{array}{l}(10) \\
\text { ulcer }\end{array}$ & $\begin{array}{c}(11) \\
\text { parkinson }\end{array}$ & $\begin{array}{c}\text { (12) } \\
\text { cataracts } \\
\end{array}$ & $\begin{array}{c}\text { (13) } \\
\text { femoralfract. }\end{array}$ & $\begin{array}{c}\text { (14) } \\
\text { othercond. }\end{array}$ & nocondi. \\
\hline Eduye & $\begin{array}{c}-0.00579^{* * *} \\
(0.00154)\end{array}$ & $\begin{array}{c}-0.00270^{\star * *} \\
(0.000579)\end{array}$ & $\begin{array}{c}-0.00488^{* * *} \\
(0.000770)\end{array}$ & $\begin{array}{c}-0.00102^{* * *} \\
(0.000312)\end{array}$ & $\begin{array}{c}-0.00392^{* * *} \\
(0.000720)\end{array}$ & $\begin{array}{c}-0.000815^{\star \star *} \\
(0.000253)\end{array}$ & $\begin{array}{c}-0.00662^{\star * *} \\
(0.00141)\end{array}$ & $\begin{array}{l}-0.000406 \\
(0.000426)\end{array}$ & $\begin{array}{l}0.00103^{\star * *} \\
(0.000363)\end{array}$ & $\begin{array}{c}-0.00139^{* * *} \\
(0.000409)\end{array}$ & $\begin{array}{c}-0.000205^{\star *} \\
(8.27 \mathrm{e}-05)\end{array}$ & $\begin{array}{l}0.000614^{* *} \\
(0.000300)\end{array}$ & $\begin{array}{c}-0.000552^{* * *} \\
(0.000130)\end{array}$ & $\begin{array}{l}-0.000118 \\
(0.000813)\end{array}$ & $\begin{array}{l}0.00430^{* * *} \\
(0.000982)\end{array}$ \\
\hline Female & $\begin{array}{l}0.0226^{\star \star \star} \\
(0.00682)\end{array}$ & $\begin{array}{l}-0.0512^{\star \star \star} \\
(0.00476)\end{array}$ & $\begin{array}{c}0.0118 \\
(0.00862)\end{array}$ & $\begin{array}{c}-0.0117^{\star \star \star} \\
(0.00179)\end{array}$ & $\begin{array}{c}-0.0232^{* * \star} \\
(0.00605)\end{array}$ & $\begin{array}{l}0.00279^{\star *} \\
(0.00140)\end{array}$ & $\begin{array}{c}0.100^{\star * *} \\
(0.00969)\end{array}$ & $\begin{array}{l}0.0567^{\star \star \star} \\
(0.00987)\end{array}$ & $\begin{array}{c}0.00805^{\star \star \star} \\
(0.00197)\end{array}$ & $\begin{array}{l}-0.0111^{* * *} \\
(0.00315)\end{array}$ & $\begin{array}{c}-0.00202^{\star \star \star} \\
(0.000585)\end{array}$ & $\begin{array}{l}0.0214^{* * *} \\
(0.00220)\end{array}$ & $\begin{array}{l}0.00365^{\star \star} \\
(0.00147)\end{array}$ & $\begin{array}{l}0.0243^{\star * *} \\
(0.00392)\end{array}$ & $\begin{array}{c}-0.0133^{\star \star *} \\
(0.00428)\end{array}$ \\
\hline Age55_59 & $\begin{array}{l}0.0452^{\star * *} \\
(0.00806)\end{array}$ & $\begin{array}{l}0.0116^{\star \star \star} \\
(0.00199)\end{array}$ & $\begin{array}{l}0.0592^{* * *} \\
(0.00764)\end{array}$ & $\begin{array}{l}0.00320^{* *} \\
(0.00128)\end{array}$ & $\begin{array}{l}0.0140^{* * *} \\
(0.00281)\end{array}$ & $\begin{array}{l}-0.00374^{*} \\
(0.00225)\end{array}$ & $\begin{array}{l}0.0465^{\star \star *} \\
(0.00499)\end{array}$ & $\begin{array}{l}0.0195^{\star * *} \\
(0.00342)\end{array}$ & $\begin{array}{c}0.00609^{* * *} \\
(0.00226)\end{array}$ & $\begin{array}{l}-0.000911 \\
(0.00317)\end{array}$ & $\begin{array}{c}0.000259 \\
(0.000448)\end{array}$ & $\begin{array}{c}0.00619^{* * *} \\
(0.00169)\end{array}$ & $\begin{array}{l}0.000750 \\
(0.00136)\end{array}$ & $\begin{array}{l}-0.00389 \\
(0.00353)\end{array}$ & $\begin{array}{c}-0.0816^{\star * *} \\
(0.0103)\end{array}$ \\
\hline Age60_64 & $\begin{array}{l}0.0525^{\star \star \star} \\
(0.00932)\end{array}$ & $\begin{array}{l}0.0293^{* * *} \\
(0.00413)\end{array}$ & $\begin{array}{l}0.111^{* * *} \\
(0.0107)\end{array}$ & $\begin{array}{c}0.00605^{\star \star *} \\
(0.00138)\end{array}$ & $\begin{array}{l}0.0269^{\star \star \star} \\
(0.00467)\end{array}$ & $\begin{array}{c}-0.00383 \\
(0.00273)\end{array}$ & $\begin{array}{l}0.0676^{\star * *} \\
(0.00776)\end{array}$ & $\begin{array}{l}0.0281^{\star \star \star} \\
(0.00502)\end{array}$ & $\begin{array}{l}0.0107^{\star * *} \\
(0.00267)\end{array}$ & $\begin{array}{l}0.000467 \\
(0.00270)\end{array}$ & $\begin{array}{l}0.0014 \\
(0.000\end{array}$ & $\begin{array}{l}0.021 \\
(0.00\end{array}$ & $\begin{array}{l}0.002 \\
(0.00\end{array}$ & $\begin{array}{l}-0.02 \\
(0.00\end{array}$ & $\begin{array}{l}-0.127^{\star \star \star} \\
(0.00995)\end{array}$ \\
\hline Age65_69 & $\begin{array}{c}0.0774^{* * *} \\
(0.0114)\end{array}$ & $\begin{array}{l}0.0513^{* * *} \\
(0.00336)\end{array}$ & $\begin{array}{l}0.154^{\star * *} \\
(0.0140)\end{array}$ & $\begin{array}{l}0.0127^{* * *} \\
(0.00265)\end{array}$ & $\begin{array}{l}0.0491^{* * *} \\
(0.00745)\end{array}$ & $\begin{array}{l}-0.00555^{\star} \\
(0.00332)\end{array}$ & $\begin{array}{c}0.0896^{\star * *} \\
(0.0124)\end{array}$ & $\begin{array}{l}0.0411^{* * *} \\
(0.00670)\end{array}$ & $\begin{array}{l}0.0149^{* * *} \\
(0.00295)\end{array}$ & $\begin{array}{l}0.000101 \\
(0.00393)\end{array}$ & $\begin{array}{l}0.00432^{\star * *} \\
(0.000891)\end{array}$ & $\begin{array}{l}0.0477^{* * *} \\
(0.00388)\end{array}$ & $\begin{array}{l}0.00548^{* *} \\
(0.00227)\end{array}$ & $\begin{array}{c}-0.0251^{* * *} \\
(0.00655)\end{array}$ & $\begin{array}{c}-0.162^{\star * *} \\
(0.0128)\end{array}$ \\
\hline Age70_74 & & $\begin{array}{l}0.0946^{* * *} \\
(0.00611)\end{array}$ & $\begin{array}{l}0.193^{* * *} \\
(0.0122)\end{array}$ & $\begin{array}{l}0.0241^{\star \star *} \\
(0.00317)\end{array}$ & $\begin{array}{l}0.0654^{* \star *} \\
(0.00735)\end{array}$ & & $\begin{array}{l}0.107^{\star * *} \\
(0.0150)\end{array}$ & $\begin{array}{l}0.0526^{\star \star *} \\
(0.00921)\end{array}$ & $\begin{array}{l}0.0270^{\star * *} \\
(0.00411)\end{array}$ & $\begin{array}{c}0.00140 \\
(0.00310)\end{array}$ & $\begin{array}{c}0.00760^{\star * \star} \\
(0.00130)\end{array}$ & $\begin{array}{l}0.0923^{* * *} \\
(0.00445)\end{array}$ & $\begin{array}{l}0.0118^{\star * *} \\
(0.00254)\end{array}$ & $\begin{array}{c}-0.0252^{\star * *} \\
(0.00821)\end{array}$ & $\begin{array}{c}-0.206^{\star \star *} \\
(0.0132)\end{array}$ \\
\hline Age75_79 & $\begin{array}{l}0.155^{\star * \star} \\
(0.0155)\end{array}$ & $\begin{array}{c}0.145^{\star * *} \\
(0.00914)\end{array}$ & $\begin{array}{l}0.205^{* * *} \\
(0.0122)\end{array}$ & $\begin{array}{l}0.0428^{* * *} \\
(0.00611)\end{array}$ & $\begin{array}{l}0.0716^{* * *} \\
(0.00881)\end{array}$ & $\begin{array}{l}-0.00160 \\
(0.00337)\end{array}$ & $\begin{array}{l}0.135^{\star * *} \\
(0.0159)\end{array}$ & $\begin{array}{l}0.0582^{* * *} \\
(0.00957)\end{array}$ & $\begin{array}{l}0.0302^{* * *} \\
(0.00504)\end{array}$ & $\begin{array}{c}0.00247 \\
(0.00415)\end{array}$ & $\begin{array}{l}0.011 \\
(0.00\end{array}$ & $\begin{array}{r}0.15 \\
(0.00\end{array}$ & $\begin{array}{l}0.0257^{* * *} \\
(0.00242)\end{array}$ & & $\begin{array}{c}-0.229^{\star * *} \\
(0.0128)\end{array}$ \\
\hline AgeAbove_80 & $\begin{array}{l}0.189^{* * *} \\
(0.0154)\end{array}$ & & $\begin{array}{l}0.184^{* * *} \\
(0.0151)\end{array}$ & $\begin{array}{l}0.0606^{* * *} \\
(0.00647)\end{array}$ & $\begin{array}{l}0.0696^{* * *} \\
(0.00935)\end{array}$ & & & $\begin{array}{c}0.0649^{* * *} \\
(0.0111)\end{array}$ & $\begin{array}{l}0.0332^{* * *} \\
(0.00589)\end{array}$ & $\begin{array}{c}0.00374 \\
(0.00494)\end{array}$ & $\begin{array}{l}0.019 \\
0.00\end{array}$ & & & & $\begin{array}{c}-0.248^{\star * *} \\
(0.0140)\end{array}$ \\
\hline Married & $\begin{array}{c}-0.0126 \\
(0.00923)\end{array}$ & $\begin{array}{c}0.0132^{*} \\
(0.00756)\end{array}$ & $\begin{array}{l}0.0259^{* *} \\
(0.0101)\end{array}$ & $\begin{array}{r}-0.000154 \\
(0.00300)\end{array}$ & $\begin{array}{l}-0.00641 \\
(0.00850)\end{array}$ & $\begin{array}{l}-0.00258 \\
(0.00316)\end{array}$ & $\begin{array}{c}-0.00518 \\
(0.00818)\end{array}$ & $\begin{array}{l}-0.00556^{*} \\
(0.00311)\end{array}$ & $\begin{array}{l}0.00703^{\star *} \\
(0.00276)\end{array}$ & $\begin{array}{l}-0.00204 \\
(0.00320)\end{array}$ & $\begin{array}{l}-0.000364 \\
(0.00128)\end{array}$ & $\begin{array}{l}-0.00692^{*} \\
(0.00416)\end{array}$ & $\begin{array}{c}-0.00324 \\
(0.00274)\end{array}$ & $\begin{array}{c}-0.0380^{* * *} \\
(0.00714)\end{array}$ & $\begin{array}{c}0.00130 \\
(0.00714)\end{array}$ \\
\hline Reg_Partnership & $\begin{array}{l}-0.0142 \\
(0.0168)\end{array}$ & $\begin{array}{c}0.0142 \\
0.0141)\end{array}$ & $\begin{array}{l}-0.0128 \\
(0.0143)\end{array}$ & $\begin{array}{r}-0.000307 \\
(0.00459)\end{array}$ & $\begin{array}{c}-0.00767 \\
(0.0142)\end{array}$ & $\begin{array}{c}0.00579 \\
(0.00680)\end{array}$ & $\begin{array}{c}-0.00538 \\
(0.0139)\end{array}$ & $\begin{array}{l}-0.00554 \\
(0.00547)\end{array}$ & $\begin{array}{l}-8.40 \mathrm{e}-05 \\
(0.00783)\end{array}$ & $\begin{array}{c}0.00246 \\
(0.00755)\end{array}$ & $\begin{array}{c}0.00271 \\
(0.00280)\end{array}$ & $\begin{array}{l}-0.000 \\
(0.00\end{array}$ & $\begin{array}{l}-0.00 \\
(0.00\end{array}$ & $\begin{array}{c}-0.0360^{* * *} \\
(0.00797)\end{array}$ & $\begin{array}{l}0.00890 \\
(0.0134)\end{array}$ \\
\hline Separated & & $\begin{array}{l}0.00536 \\
(0.0121)\end{array}$ & $\begin{array}{c}0.0157 \\
(0.0182)\end{array}$ & $\begin{array}{l}0.0175^{\star * *} \\
(0.00592)\end{array}$ & $\begin{array}{c}0.0144 \\
(0.0123)\end{array}$ & $\begin{array}{l}-0.00371 \\
(0.00449)\end{array}$ & $\begin{array}{c}0.00819 \\
(0.00977)\end{array}$ & $\begin{array}{l}-0.00429 \\
(0.00671)\end{array}$ & $\begin{array}{c}0.0113 \\
(0.00827)\end{array}$ & $\begin{array}{c}0.0122 \\
(0.00899)\end{array}$ & $\begin{array}{c}0.00275 \\
(0.00328)\end{array}$ & $\begin{array}{r}0.00 \\
(0.00\end{array}$ & $\begin{array}{l}-0.00 \\
(0.00\end{array}$ & $\begin{array}{r}-0.03 \\
(0.0\end{array}$ & $\begin{array}{l}-0.0146 \\
(0.0201)\end{array}$ \\
\hline 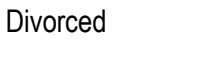 & $\begin{array}{l}0.0268^{* *} \\
(0.0116)\end{array}$ & $\begin{array}{c}0.0210^{* *} \\
(0.00899)\end{array}$ & $\begin{array}{l}-0.00 \\
(0.008\end{array}$ & $\begin{array}{r}0.001 \\
(0.003\end{array}$ & & & & & & & & & & & $\begin{array}{l}-0.0221^{* * *} \\
(0.00792)\end{array}$ \\
\hline Widow & $\begin{array}{l}0.00504 \\
(0.0104)\end{array}$ & $\begin{array}{l}0.0227^{\star \star *} \\
(0.00610)\end{array}$ & $\begin{array}{c}0.0607^{\star * *} \\
(0.0114)\end{array}$ & $\begin{array}{l}0.000114 \\
(0.00350)\end{array}$ & $\begin{array}{c}0.00865 \\
(0.00891)\end{array}$ & $\begin{array}{l}0.000 \\
(0.003\end{array}$ & $\begin{array}{l}0.0 \\
(0.1\end{array}$ & $\begin{array}{r}0.005 \\
(0.003\end{array}$ & $\begin{array}{l}0.000 \\
(0.00\end{array}$ & $\begin{array}{r}0.001 \\
(0.004\end{array}$ & $\begin{array}{l}-0.00 \\
(0.00\end{array}$ & $\begin{array}{l}0.0 \\
(0.0\end{array}$ & $\begin{array}{l}0.0 \\
10\end{array}$ & $\begin{array}{l}-0.0 \\
(0.0\end{array}$ & $\begin{array}{l}-0.02 \\
(0.00\end{array}$ \\
\hline $\mathrm{R}$ & $\begin{array}{c}-0.180^{* * *} \\
(0.0183)\end{array}$ & $\begin{array}{l}-0.0347^{* * *} \\
(0.00748)\end{array}$ & $-0.0191^{* *}$ & $\begin{array}{l}-0.0283^{* * *} \\
(0.00476)\end{array}$ & $-0.0282^{* * *}$ & $-0.0113^{* *}$ & $-0.0679^{* * *}$ & $\begin{array}{l}-0.0241^{* * *} \\
(0.00531)\end{array}$ & $\begin{array}{l}-0.0124^{* * *} \\
(0.00405)\end{array}$ & $-0.0213^{* * *}$ & $\begin{array}{l}-0.00290^{*} \\
(0.00154)\end{array}$ & $\begin{array}{c}-0.00938^{* * *} \\
(0.00331)\end{array}$ & $\begin{array}{l}-0.0143^{* * *} \\
(0.00328)\end{array}$ & $\begin{array}{c}-0.0944^{* * *} \\
(0.00532)\end{array}$ & $\begin{array}{l}0.0864^{* * *} \\
(0.00880)\end{array}$ \\
\hline En & $\begin{array}{c}-0.287^{\star * *} \\
(0.0151)\end{array}$ & $\begin{array}{c}-0.0717^{\star * *} \\
(0.00826)\end{array}$ & $\begin{array}{c}-0.0597^{\star * \star} \\
(0.0110)\end{array}$ & $\begin{array}{c}-0.0456^{\star \star *} \\
(0.00494)\end{array}$ & $\begin{array}{c}-0.0469^{* * *} \\
(0.00565)\end{array}$ & $\begin{array}{c}-0.0190^{\star * *} \\
(0.00486)\end{array}$ & $\begin{array}{c}-0.0980^{\star * *} \\
(0.00834)\end{array}$ & $\begin{array}{l}-0.0241^{* * *} \\
(0.00472)\end{array}$ & $\begin{array}{c}-0.0366^{\star \star *} \\
(0.00283)\end{array}$ & $\begin{array}{l}-0.0331^{* * *} \\
(0.00603)\end{array}$ & $\begin{array}{c}-0.00461^{* \star *} \\
(0.00131)\end{array}$ & $\begin{array}{c}-0.0221^{\star * *} \\
(0.00219)\end{array}$ & $\begin{array}{c}-0.0173^{\star \star \star} \\
(0.00257)\end{array}$ & $\begin{array}{l}-0.131^{\star \star *} \\
(0.00883)\end{array}$ & $\begin{array}{l}0.178^{* * *} \\
(0.0110)\end{array}$ \\
\hline Homemaker & $\begin{array}{l}-0.212^{* * *} \\
(0.0171)\end{array}$ & $\begin{array}{l}-0.0508^{* * *} \\
(0.00842)\end{array}$ & $\begin{array}{c}-0.0267^{* *} \\
(0.0113)\end{array}$ & $\begin{array}{l}-0.0357^{* * *} \\
(0.00591)\end{array}$ & $\begin{array}{l}-0.0224^{* * *} \\
(0.00715)\end{array}$ & $\begin{array}{c}-0.0132^{* * *} \\
(0.00433)\end{array}$ & $\begin{array}{c}-0.0627^{\star * *} \\
(0.0123)\end{array}$ & $\begin{array}{l}-0.00957 \\
(0.00802)\end{array}$ & $\begin{array}{l}-0.0220^{\star * *} \\
(0.00393)\end{array}$ & $\begin{array}{l}-0.0291^{* * *} \\
(0.00672)\end{array}$ & $\begin{array}{c}-0.00484^{* * *} \\
(0.00177)\end{array}$ & $\begin{array}{l}-0.0153^{* * *} \\
(0.00345)\end{array}$ & $\begin{array}{l}-0.0148^{* * *} \\
(0.00298)\end{array}$ & $\begin{array}{l}-0.107^{\star * *} \\
(0.00668)\end{array}$ & $\begin{array}{l}0.105^{\star * *} \\
(0.0119)\end{array}$ \\
\hline Other_Job & $\begin{array}{c}-0.187^{\star * *} \\
(0.0312)\end{array}$ & $\begin{array}{c}-0.0356^{* * *} \\
(0.0121)\end{array}$ & $\begin{array}{c}-0.0369^{* *} \\
(0.0162)\end{array}$ & $\begin{array}{c}-0.0195^{\star \star *} \\
(0.00679)\end{array}$ & $\begin{array}{c}-0.0308^{* * *} \\
(0.0119)\end{array}$ & $\begin{array}{c}-0.0239^{\star * *} \\
(0.00754)\end{array}$ & $\begin{array}{c}-0.0645^{\star \star *} \\
(0.0119)\end{array}$ & $\begin{array}{l}-0.0244^{\star * *} \\
(0.00737)\end{array}$ & $\begin{array}{c}-0.0170^{*} \\
(0.00918)\end{array}$ & $\begin{array}{c}-0.0299^{* * *} \\
(0.00689)\end{array}$ & $\begin{array}{l}-0.005 \\
(0.002\end{array}$ & $\begin{array}{l}-0.01 \\
(0.00\end{array}$ & $\begin{array}{c}-0.0120^{\star \star \star} \\
(0.00443)\end{array}$ & $\begin{array}{c}-0.0827^{\star * *} \\
(0.0128)\end{array}$ & $\begin{array}{c}0.0975^{\star \star *} \\
(0.0128)\end{array}$ \\
\hline N_Children & $\begin{array}{l}-0.00366^{*} \\
(0.00188)\end{array}$ & $\begin{array}{l}0.000351 \\
(0.00129)\end{array}$ & $\begin{array}{c}-0.00203 \\
(0.00133)\end{array}$ & $\begin{array}{c}0.00120 \\
(0.000893)\end{array}$ & $\begin{array}{l}0.00295^{\star *} \\
(0.00140)\end{array}$ & $\begin{array}{l}0.000865^{*} \\
(0.000491)\end{array}$ & $\begin{array}{l}0.000465 \\
(0.00202)\end{array}$ & $\begin{array}{l}-0.00173^{* *} \\
(0.000761)\end{array}$ & $\begin{array}{c}-0.00238^{* * *} \\
(0.000819)\end{array}$ & $\begin{array}{l}-0.000612 \\
(0.00104)\end{array}$ & $\begin{array}{c}0.000145 \\
(0.000334)\end{array}$ & $\begin{array}{l}-0.00256^{*} \\
(0.00137)\end{array}$ & $\begin{array}{l}-0.000395 \\
(0.000590)\end{array}$ & $\begin{array}{c}-0.00193 \\
(0.00142)\end{array}$ & $\begin{array}{c}0.00485^{\star * *} \\
(0.00174)\end{array}$ \\
\hline N_Grandchildren & $\begin{array}{l}0.00531^{* * *} \\
(0.000794)\end{array}$ & $\begin{array}{l}0.00274^{\star * *} \\
(0.000697)\end{array}$ & $\begin{array}{l}0.00406^{* * *} \\
(0.000649)\end{array}$ & $\begin{array}{c}0.000363 \\
(0.000452)\end{array}$ & $\begin{array}{l}0.00200^{* * *} \\
(0.000593)\end{array}$ & $\begin{array}{c}0.000686^{* * *} \\
(0.000250)\end{array}$ & $\begin{array}{l}0.00281^{* * *} \\
(0.000906)\end{array}$ & $\begin{array}{c}0.000787^{*} \\
(0.000439)\end{array}$ & $\begin{array}{l}-1.81 \mathrm{e}-05 \\
(0.000256)\end{array}$ & $\begin{array}{l}0.000931^{\text {** }} \\
(0.000472)\end{array}$ & $\begin{array}{c}1.25 \mathrm{e}-05 \\
(0.000144)\end{array}$ & $\begin{array}{l}0.000876^{*} \\
(0.000452)\end{array}$ & $\begin{array}{c}0.000107 \\
(0.000252)\end{array}$ & $\begin{array}{c}0.000529 \\
(0.000561)\end{array}$ & $\begin{array}{c}-0.00487^{\star * *} \\
(0.000825)\end{array}$ \\
\hline Logincome & $\begin{array}{c}0.00334 \\
(0.00280)\end{array}$ & $\begin{array}{l}7.42 \mathrm{e}-05 \\
(0.00115)\end{array}$ & $\begin{array}{l}-0.00244 \\
(0.00184)\end{array}$ & $\begin{array}{c}0.000873 \\
(0.000588)\end{array}$ & $\begin{array}{l}-0.000839 \\
(0.00120)\end{array}$ & $\begin{array}{l}0.00208^{* *} \\
(0.00105)\end{array}$ & $\begin{array}{c}0.00134 \\
(0.00155)\end{array}$ & $\begin{array}{l}0.000454 \\
(0.00177)\end{array}$ & $\begin{array}{c}0.00380^{\star * \star} \\
(0.00102)\end{array}$ & $\begin{array}{l}-8.09 \mathrm{e}-05 \\
(0.00103)\end{array}$ & $\begin{array}{c}0.000238 \\
(0.000183)\end{array}$ & $\begin{array}{l}0.00257^{*} \\
(0.00150)\end{array}$ & $\begin{array}{c}0.000614 \\
(0.000506)\end{array}$ & $\begin{array}{c}0.00601^{* * *} \\
(0.00165)\end{array}$ & $\begin{array}{c}-0.00584^{* *} \\
(0.00235)\end{array}$ \\
\hline
\end{tabular}

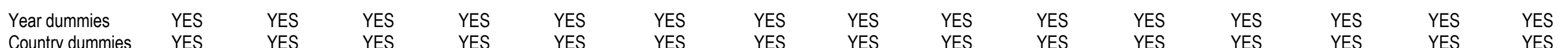


Table A4b - Education years and specific diseases

\begin{tabular}{|c|c|c|c|c|c|c|c|c|c|c|c|c|c|c|c|}
\hline VARIABLES & $\begin{array}{c}1) \\
\text { longterm } \\
\text { illness }\end{array}$ & $\begin{array}{c}(2) \\
\text { heart } \\
\text { attack }\end{array}$ & $\begin{array}{c}(3) \\
\text { hyper } \\
\text { tension }\end{array}$ & $\begin{array}{c}(4) \\
\text { stroke }\end{array}$ & $\begin{array}{c}(5) \\
\text { diabetes }\end{array}$ & $\begin{array}{c}\text { (6) } \\
\text { asthma }\end{array}$ & $\begin{array}{c}\text { (7) } \\
\text { arthritis }\end{array}$ & $\begin{array}{c}(8) \\
\text { osteopor }\end{array}$ & $\begin{array}{c}\text { (9) } \\
\text { cancer }\end{array}$ & $\begin{array}{l}(10) \\
\text { ulcer }\end{array}$ & $\begin{array}{c}(11) \\
\text { parkinson }\end{array}$ & $\begin{array}{c}\text { (12) } \\
\text { cataracts }\end{array}$ & $\begin{array}{c}\text { (13) } \\
\text { femoralfr. }\end{array}$ & $\begin{array}{c}\text { (14) } \\
\text { othercon. }\end{array}$ & $\begin{array}{c}\text { (15) } \\
\text { nocondi. }\end{array}$ \\
\hline Eduyears & $\begin{array}{c}-0.00390^{* * *} \\
(0.00143)\end{array}$ & $\begin{array}{c}-0.00205^{* * *} \\
(0.000471)\end{array}$ & $\begin{array}{l}-0.00337^{* * *} \\
(0.000682)\end{array}$ & $\begin{array}{c}-0.000678^{* *} \\
(0.000271)\end{array}$ & $\begin{array}{c}-0.00297^{* * *} \\
(0.000665)\end{array}$ & $\begin{array}{c}-0.000698^{* * *} \\
(0.000237)\end{array}$ & $\begin{array}{c}-0.00592^{* * *} \\
(0.00136)\end{array}$ & $\begin{array}{l}-0.000488 \\
(0.000437)\end{array}$ & $\begin{array}{l}0.00108^{* * *} \\
0.000376)\end{array}$ & $\begin{array}{c}-0.00133^{* * *} \\
(0.000369)\end{array}$ & $\begin{array}{c}-0.000136^{*} \\
(7.92 \mathrm{e}-05)\end{array}$ & $\begin{array}{l}0.000715^{\star *} \\
(0.000296)\end{array}$ & $\begin{array}{c}-0.000478^{* * *} \\
(0.000136)\end{array}$ & $\begin{array}{c}0.000110 \\
(0.000806)\end{array}$ & $\begin{array}{l}0.00318^{* * *} \\
(0.000899)\end{array}$ \\
\hline \multicolumn{16}{|l|}{ DRINKING } \\
\hline 5or6days & $\begin{array}{c}-0.0228^{* * *} \\
(0.00660)\end{array}$ & $\begin{array}{c}-0.0279^{* * *} \\
(0.00663)\end{array}$ & $\begin{array}{l}0.000590 \\
(0.00688)\end{array}$ & $\begin{array}{c}-0.0174^{* * *} \\
(0.00446)\end{array}$ & $\begin{array}{c}-0.0175^{\star * *} \\
(0.00248)\end{array}$ & $\begin{array}{c}-0.00384^{* *} \\
(0.00164)\end{array}$ & $\begin{array}{c}0.00118 \\
(0.00548)\end{array}$ & $\begin{array}{c}-0.00201 \\
(0.00295)\end{array}$ & $\begin{array}{c}-0.00441^{* *} \\
(0.00203)\end{array}$ & $\begin{array}{c}-0.00435 \\
(0.00367)\end{array}$ & $\begin{array}{c}-0.00306^{* * *} \\
(0.000860)\end{array}$ & $\begin{array}{c}0.00503 \\
(0.00448)\end{array}$ & $\begin{array}{l}-0.00106 \\
(0.00162)\end{array}$ & $\begin{array}{c}0.00686 \\
(0.00499)\end{array}$ & $\begin{array}{l}-0.00411 \\
(0.00642)\end{array}$ \\
\hline 3or4days_week & $\begin{array}{c}-0.0450^{* * *} \\
(0.00764)\end{array}$ & $\begin{array}{c}-0.0293^{* * *} \\
(0.00744)\end{array}$ & $\begin{array}{l}-0.00327 \\
(0.00608)\end{array}$ & $\begin{array}{c}-0.0183^{\star * *} \\
(0.00418)\end{array}$ & $\begin{array}{l}-0.0271^{* * *} \\
(0.00355)\end{array}$ & $\begin{array}{c}-0.00441^{* *} \\
(0.00218)\end{array}$ & $\begin{array}{c}0.00500 \\
(0.00506)\end{array}$ & $\begin{array}{c}-0.00317 \\
(0.00327)\end{array}$ & $\begin{array}{c}-0.00396 \\
(0.00249)\end{array}$ & $\begin{array}{l}-0.00265 \\
(0.00320)\end{array}$ & $\begin{array}{c}-0.00336^{* * *} \\
(0.00107)\end{array}$ & $\begin{array}{c}0.00544 \\
(0.00377)\end{array}$ & $\begin{array}{l}-0.00154 \\
(0.00132)\end{array}$ & $\begin{array}{c}-0.000340 \\
(0.00466)\end{array}$ & $\begin{array}{c}0.00661 \\
(0.00550)\end{array}$ \\
\hline 1or2 & $\begin{array}{c}-0.0652^{* * *} \\
(0.00638)\end{array}$ & $\begin{array}{c}-0.0326^{* * *} \\
(0.00626)\end{array}$ & $\begin{array}{c}-0.0114^{*} \\
(0.00591)\end{array}$ & $\begin{array}{l}-0.0206^{* * *} \\
(0.00426)\end{array}$ & $\begin{array}{l}-0.0337^{* * *} \\
(0.00443)\end{array}$ & $\begin{array}{c}-0.00428^{* * *} \\
(0.00161)\end{array}$ & $\begin{array}{l}0.000402 \\
(0.00543)\end{array}$ & $\begin{array}{l}-0.00140 \\
(0.00327)\end{array}$ & $\begin{array}{c}-0.00705^{\star *} \\
(0.00298)\end{array}$ & $\begin{array}{c}-0.00362 \\
(0.00394)\end{array}$ & $\begin{array}{l}-0.00415^{\star * *} \\
(0.000956)\end{array}$ & $\begin{array}{c}0.00428 \\
(0.00398)\end{array}$ & $\begin{array}{r}-0.000817 \\
(0.00153)\end{array}$ & $\begin{array}{l}-0.00981^{*} \\
(0.00572)\end{array}$ & $\begin{array}{l}0.00935^{\star} \\
(0.00565)\end{array}$ \\
\hline 1or2_month & $\begin{array}{c}-0.0587^{* * *} \\
(0.00914)\end{array}$ & $\begin{array}{c}-0.0305^{* * *} \\
(0.00724)\end{array}$ & $\begin{array}{l}-0.00909^{*} \\
(0.00517)\end{array}$ & $\begin{array}{c}-0.0236^{* * *} \\
(0.00439)\end{array}$ & $\begin{array}{l}-0.0365^{\star * *} \\
(0.00486)\end{array}$ & $\begin{array}{c}-0.00518^{* *} \\
(0.00212)\end{array}$ & $\begin{array}{c}-0.00124 \\
(0.00685)\end{array}$ & $\begin{array}{c}-0.00690^{* *} \\
(0.00338)\end{array}$ & $\begin{array}{c}-0.00857^{* *} \\
(0.00357)\end{array}$ & $\begin{array}{l}-0.00474 \\
(0.00333)\end{array}$ & $\begin{array}{c}-0.00392^{* * *} \\
(0.00107)\end{array}$ & $\begin{array}{c}0.00472 \\
(0.00450)\end{array}$ & $\begin{array}{l}3.94 \mathrm{e}-05 \\
(0.00202)\end{array}$ & $\begin{array}{l}-0.00780 \\
(0.00642)\end{array}$ & $\begin{array}{c}0.0151 \\
(0.00935)\end{array}$ \\
\hline$<1$ _month & $\begin{array}{c}-0.0744^{* * *} \\
(0.0106)\end{array}$ & $\begin{array}{c}-0.0340^{* * *} \\
(0.00659)\end{array}$ & $\begin{array}{l}-0.0177^{\star *} \\
(0.00873)\end{array}$ & $\begin{array}{c}-0.0233^{* * *} \\
(0.00389)\end{array}$ & $\begin{array}{c}-0.0455^{\star * *} \\
(0.00489)\end{array}$ & $\begin{array}{c}-0.00586^{* *} \\
(0.00246)\end{array}$ & $\begin{array}{c}-0.00161 \\
(0.00848)\end{array}$ & $\begin{array}{l}-0.00523 \\
(0.00391)\end{array}$ & $\begin{array}{c}-0.00735 \\
(0.00469)\end{array}$ & $\begin{array}{c}-0.00226 \\
(0.00583)\end{array}$ & $\begin{array}{c}-0.00561^{* * *} \\
(0.00126)\end{array}$ & $\begin{array}{c}0.00435 \\
(0.00604)\end{array}$ & $\begin{array}{l}-0.00147 \\
(0.00265)\end{array}$ & $\begin{array}{c}-0.00857 \\
(0.00689)\end{array}$ & $\begin{array}{l}0.0232^{* *} \\
(0.0113)\end{array}$ \\
\hline 0_in_3months & $\begin{array}{c}-0.0579^{* * *} \\
(0.00786)\end{array}$ & $\begin{array}{l}-0.0326^{* * *} \\
(0.00563)\end{array}$ & $\begin{array}{l}-0.00257 \\
(0.00570)\end{array}$ & $\begin{array}{c}-0.0179^{* * *} \\
(0.00353)\end{array}$ & $\begin{array}{c}-0.0413^{* * *} \\
(0.00596)\end{array}$ & $\begin{array}{l}-0.00161^{* *} \\
(0.000782)\end{array}$ & $\begin{array}{c}0.00198 \\
(0.00650)\end{array}$ & $\begin{array}{l}-0.00435^{*} \\
(0.00242)\end{array}$ & $\begin{array}{c}-0.00619^{* *} \\
(0.00259)\end{array}$ & $\begin{array}{l}-0.00491 \\
(0.00318)\end{array}$ & $\begin{array}{c}-0.00413^{* * *} \\
(0.000977)\end{array}$ & $\begin{array}{l}0.0108^{* * *} \\
(0.00313)\end{array}$ & $\begin{array}{l}3.60 \mathrm{e}-05 \\
(0.00167)\end{array}$ & $\begin{array}{c}-0.00584 \\
(0.00568)\end{array}$ & $\begin{array}{c}0.00279 \\
(0.00696)\end{array}$ \\
\hline \multicolumn{16}{|l|}{ SPORT } \\
\hline 1_week & $\begin{array}{c}0.00977^{*} \\
(0.00593)\end{array}$ & $\begin{array}{c}0.00504 \\
(0.00311)\end{array}$ & $\begin{array}{l}0.0151^{* * *} \\
(0.00528)\end{array}$ & $\begin{array}{l}0.000256 \\
(0.00132)\end{array}$ & $\begin{array}{l}0.00476^{*} \\
(0.00270)\end{array}$ & $\begin{array}{l}0.000633 \\
(0.00160)\end{array}$ & $\begin{array}{c}0.00422 \\
(0.00346)\end{array}$ & $\begin{array}{c}0.00138 \\
(0.00205)\end{array}$ & $\begin{array}{c}0.00241 \\
(0.00250)\end{array}$ & $\begin{array}{l}0.000463 \\
(0.00244)\end{array}$ & $\begin{array}{c}0.000310 \\
(0.000452)\end{array}$ & $\begin{array}{c}0.00258 \\
(0.00310)\end{array}$ & $\begin{array}{c}-0.000190 \\
(0.000907)\end{array}$ & $\begin{array}{r}0.00 \\
(0.00\end{array}$ & $\begin{array}{l}-0.0158^{* *} \\
(0.00666)\end{array}$ \\
\hline 1or3_month & $\begin{array}{l}0.0443^{* * *} \\
(0.00800)\end{array}$ & $\begin{array}{l}0.0183^{* * *} \\
(0.00480)\end{array}$ & $\begin{array}{c}0.0176^{* *} \\
(0.00689)\end{array}$ & $\begin{array}{c}0.00288 \\
(0.00211)\end{array}$ & $\begin{array}{l}0.00826^{* *} \\
(0.00357)\end{array}$ & $\begin{array}{c}0.00281 \\
(0.00183)\end{array}$ & $\begin{array}{c}0.00533 \\
(0.00527)\end{array}$ & $\begin{array}{l}0.000660 \\
(0.00230)\end{array}$ & $\begin{array}{c}0.00101 \\
(0.00268)\end{array}$ & $\begin{array}{c}0.00197 \\
(0.00235)\end{array}$ & $\begin{array}{c}0.000347 \\
(0.000548)\end{array}$ & $\begin{array}{c}0.00128 \\
(0.00256)\end{array}$ & $\begin{array}{l}0.000349 \\
(0.00142)\end{array}$ & $\begin{array}{l}0.0127^{* \star *} \\
(0.00464)\end{array}$ & $\begin{array}{c}-0.0369^{* * *} \\
(0.00657)\end{array}$ \\
\hline $\begin{array}{r}\text { Hardlyever_} \\
\text { never }\end{array}$ & $\begin{array}{c}0.143^{* * *} \\
(0.00949)\end{array}$ & $\begin{array}{l}0.0550^{\star * \star} \\
(0.00653)\end{array}$ & $\begin{array}{l}0.0491^{* * *} \\
(0.00794)\end{array}$ & $\begin{array}{l}0.0253^{* * *} \\
(0.00524)\end{array}$ & $\begin{array}{l}0.0296^{* * *} \\
(0.00436)\end{array}$ & $\begin{array}{l}0.0119^{* * *} \\
(0.00215)\end{array}$ & $\begin{array}{l}0.0530^{* * *} \\
(0.00704)\end{array}$ & $\begin{array}{l}0.0113^{* * *} \\
(0.00342)\end{array}$ & $\begin{array}{l}0.0203^{\star * *} \\
(0.00152)\end{array}$ & $\begin{array}{l}0.0109^{\star * *} \\
(0.00255)\end{array}$ & $\begin{array}{l}0.00482^{\star * *} \\
(0.000673)\end{array}$ & $\begin{array}{l}0.0182^{* * *} \\
(0.00318)\end{array}$ & $\begin{array}{c}0.00872^{\star * *} \\
(0.00143)\end{array}$ & $\begin{array}{l}0.0351^{* * *} \\
(0.00380)\end{array}$ & $\begin{array}{c}-0.0828^{* * *} \\
(0.00682)\end{array}$ \\
\hline Smoking & $\begin{array}{l}-0.00863^{*} \\
(0.00494)\end{array}$ & $\begin{array}{l}-0.0202^{* * *} \\
(0.00213)\end{array}$ & $\begin{array}{c}-0.0382^{\star * *} \\
(0.00564)\end{array}$ & $\begin{array}{c}-0.00156 \\
(0.00172)\end{array}$ & $\begin{array}{c}-0.00994^{* \star *} \\
(0.00312)\end{array}$ & $\begin{array}{c}-0.00250 \\
(0.00186)\end{array}$ & $\begin{array}{l}-0.00111 \\
(0.00378)\end{array}$ & $\begin{array}{l}0.000370 \\
(0.00241)\end{array}$ & $\begin{array}{c}-0.00452^{\star *} \\
(0.00218)\end{array}$ & $\begin{array}{l}0.0200^{* * *} \\
(0.00325)\end{array}$ & $\begin{array}{l}-0.00209^{* * *} \\
(0.000499)\end{array}$ & $\begin{array}{c}-0.000652 \\
(0.00257)\end{array}$ & $\begin{array}{l}0.000402 \\
(0.00115)\end{array}$ & $\begin{array}{c}0.00423 \\
(0.00276)\end{array}$ & $\begin{array}{c}0.00299 \\
(0.00355)\end{array}$ \\
\hline $\begin{array}{l}\text { Overweight_ } \\
\text { Obese }\end{array}$ & $\begin{array}{l}0.0645^{\star * *} \\
(0.00464)\end{array}$ & $\begin{array}{l}0.0200^{\star * *} \\
(0.00424)\end{array}$ & $\begin{array}{c}0.142^{* * *} \\
(0.00878)\end{array}$ & $\begin{array}{l}0.000169 \\
(0.00112)\end{array}$ & $\begin{array}{l}0.0517^{\star * *} \\
(0.00535)\end{array}$ & $\begin{array}{c}0.00463^{* * *} \\
(0.00163)\end{array}$ & $\begin{array}{l}0.0448^{* * *} \\
(0.00434)\end{array}$ & $\begin{array}{c}-0.0107^{* * *} \\
(0.00285)\end{array}$ & $\begin{array}{c}-0.00529^{* * *} \\
(0.00156)\end{array}$ & $\begin{array}{c}-0.00111 \\
(0.00273)\end{array}$ & $\begin{array}{l}-0.00171^{* * *} \\
(0.000494)\end{array}$ & $\begin{array}{l}0.00420^{* *} \\
(0.00197)\end{array}$ & $\begin{array}{c}-0.000777 \\
(0.000830)\end{array}$ & $\begin{array}{c}0.00232 \\
(0.00347)\end{array}$ & $\begin{array}{l}-0.0787^{* * *} \\
(0.00297)\end{array}$ \\
\hline Female & $\begin{array}{c}0.00540 \\
(0.00748)\end{array}$ & $\begin{array}{l}-0.0613^{\star \star \star} \\
(0.00349)\end{array}$ & $\begin{array}{l}0.0180^{\star * *} \\
(0.00553)\end{array}$ & $\begin{array}{c}-0.0173^{* * *} \\
(0.00280)\end{array}$ & $\begin{array}{c}-0.0300^{* \star *} \\
(0.00494)\end{array}$ & $\begin{array}{c}0.00173 \\
(0.00147)\end{array}$ & $\begin{array}{l}0.101^{* \star *} \\
(0.0103)\end{array}$ & $\begin{array}{l}0.0540^{* \star *} \\
(0.00947)\end{array}$ & $\begin{array}{l}0.00435^{\star *} \\
(0.00201)\end{array}$ & $\begin{array}{l}-0.0109^{\star * *} \\
(0.00302)\end{array}$ & $\begin{array}{c}-0.00329^{\star * *} \\
(0.000627)\end{array}$ & $\begin{array}{l}0.0224^{\star * \star} \\
(0.00216)\end{array}$ & $\begin{array}{l}0.00278^{*} \\
(0.00152)\end{array}$ & $\begin{array}{l}0.0206^{* * *} \\
(0.00388)\end{array}$ & $\begin{array}{c}-0.0133^{* \star *} \\
(0.00431)\end{array}$ \\
\hline Age55_59 & $\begin{array}{l}0.0402^{\star * *} \\
(0.00816)\end{array}$ & $\begin{array}{c}0.00894^{\star * *} \\
(0.00182)\end{array}$ & $\begin{array}{l}0.0524^{\star * *} \\
(0.00764)\end{array}$ & $\begin{array}{l}0.00257^{\star *} \\
(0.00119)\end{array}$ & $\begin{array}{l}0.0114^{* \star *} \\
(0.00264)\end{array}$ & $\begin{array}{l}-0.00422^{*} \\
(0.00226)\end{array}$ & $\begin{array}{l}0.0424^{* * *} \\
(0.00522)\end{array}$ & $\begin{array}{l}0.0197^{\star \star *} \\
(0.00344)\end{array}$ & $\begin{array}{l}0.00526^{* *} \\
(0.00223)\end{array}$ & $\begin{array}{l}-0.000953 \\
(0.00330)\end{array}$ & $\begin{array}{c}0.000245 \\
(0.000451)\end{array}$ & $\begin{array}{c}0.00561^{* \star *} \\
(0.00163)\end{array}$ & $\begin{array}{l}0.000450 \\
(0.00141)\end{array}$ & $\begin{array}{l}-0.00555 \\
(0.00369)\end{array}$ & $\begin{array}{c}-0.0768^{\star \star \star} \\
(0.0105)\end{array}$ \\
\hline Age60_64 & $\begin{array}{l}0.0428^{\star * \star} \\
(0.00864)\end{array}$ & $\begin{array}{l}0.0242^{\star \star \star} \\
(0.00368)\end{array}$ & $\begin{array}{l}0.100^{* * *} \\
(0.0102)\end{array}$ & $\begin{array}{c}0.00472^{\star \star \star} \\
(0.00133)\end{array}$ & $\begin{array}{l}0.0224^{* * \star} \\
(0.00438)\end{array}$ & $\begin{array}{l}-0.00474^{*} \\
(0.00287)\end{array}$ & $\begin{array}{l}0.0613^{* * *} \\
(0.00707)\end{array}$ & $\begin{array}{l}0.0275^{\star \star \star} \\
(0.00479)\end{array}$ & $\begin{array}{c}0.00941^{* \star *} \\
(0.00256)\end{array}$ & $\begin{array}{c}0.00116 \\
(0.00298)\end{array}$ & $\begin{array}{c}0.00123^{* *} \\
(0.000527)\end{array}$ & $\begin{array}{l}0.0206^{\star * *} \\
(0.00294)\end{array}$ & $\begin{array}{c}0.00214 \\
(0.00160)\end{array}$ & $\begin{array}{c}-0.0253^{\star * *} \\
(0.00456)\end{array}$ & $\begin{array}{l}-0.121^{* * *} \\
(0.00982)\end{array}$ \\
\hline Age65_69 & $\begin{array}{c}0.0624^{* * *} \\
(0.0105)\end{array}$ & $\begin{array}{l}0.0438^{\star * *} \\
(0.00304)\end{array}$ & $\begin{array}{l}0.141^{* * *} \\
(0.0129)\end{array}$ & $\begin{array}{l}0.0104^{* * *} \\
(0.00235)\end{array}$ & $\begin{array}{l}0.0427^{* * *} \\
(0.00692)\end{array}$ & $\begin{array}{c}-0.00724^{* *} \\
(0.00347)\end{array}$ & $\begin{array}{c}0.0822^{* * *} \\
(0.0121)\end{array}$ & $\begin{array}{l}0.0405^{\star * \star} \\
(0.00631)\end{array}$ & $\begin{array}{l}0.0123^{\star * *} \\
(0.00308)\end{array}$ & $\begin{array}{c}0.00154 \\
(0.00387)\end{array}$ & $\begin{array}{l}0.00367^{* * *} \\
(0.0000832)\end{array}$ & $\begin{array}{l}0.0468^{* * *} \\
(0.00399)\end{array}$ & $\begin{array}{l}0.00430^{*} \\
(0.00223)\end{array}$ & $\begin{array}{l}-0.0266^{* * *} \\
(0.00626)\end{array}$ & $\begin{array}{c}-0.153^{* * *} \\
(0.0125)\end{array}$ \\
\hline - 74 & $\begin{array}{c}0.0914^{* * *} \\
(0.0117)\end{array}$ & $\begin{array}{l}0.0812^{\star * \star} \\
(0.00498)\end{array}$ & $\begin{array}{l}0.178^{\star \star \star} \\
(0.0112)\end{array}$ & $\begin{array}{l}0.0182^{\star \star *} \\
(0.00285)\end{array}$ & $\begin{array}{l}0.0558^{\star * \star} \\
(0.00609)\end{array}$ & $\begin{array}{c}-0.00364 \\
(0.00385)\end{array}$ & $\begin{array}{c}0.0965^{\star * *} \\
(0.0150)\end{array}$ & $\begin{array}{l}0.0506^{\star * \star} \\
(0.00864)\end{array}$ & $\begin{array}{l}0.0227^{\star \star \star} \\
(0.00396)\end{array}$ & $\begin{array}{c}0.00275 \\
(0.00327)\end{array}$ & $\begin{array}{c}0.00624^{\star \star *} \\
(0.00123)\end{array}$ & $\begin{array}{l}0.0906^{\star * *} \\
(0.00450)\end{array}$ & $\begin{array}{c}0.00972^{\star * *} \\
(0.00239)\end{array}$ & $\begin{array}{c}-0.0287^{\star * *} \\
(0.00772)\end{array}$ & $\begin{array}{c}-0.192^{* * *} \\
(0.0134)\end{array}$ \\
\hline Age75_79 & $0.116^{*}$ & $0.125^{\star * *}$ & $0.189^{* \star *}$ & $0.0346^{* * *}$ & $0.0595^{\star * *}$ & -0.00503 & $0.121^{* * *}$ & $0.0549^{\star * *}$ & $0.0235^{\star * *}$ & 0.00321 & $0.00947^{* * *}$ & $0.152^{* * *}$ & $0.0225^{\star * *}$ & $-0.0219^{\star * *}$ & $11^{* * *}$ \\
\hline
\end{tabular}




\begin{tabular}{|c|c|c|c|c|c|c|c|c|c|c|c|c|c|c|c|}
\hline & $(0.0152)$ & $(0.00710)$ & $(0.0113)$ & $(0.00486)$ & $(0.00762)$ & $(0.00361)$ & $(0.0156)$ & $(0.00866)$ & $(0.00512)$ & $(0.00456)$ & $(0.00201)$ & $(0.00743)$ & $(0.00240)$ & $(0.00790)$ & $(0.0136)$ \\
\hline AgeAbove_80 & $\begin{array}{l}0.134^{* * *} \\
(0.0156)\end{array}$ & $\begin{array}{c}0.148^{* * *} \\
(0.00995)\end{array}$ & $\begin{array}{l}0.174^{* * \star} \\
(0.0143)\end{array}$ & $\begin{array}{l}0.0473^{\star * *} \\
(0.00562)\end{array}$ & $\begin{array}{l}0.0565^{\star * *} \\
(0.00934)\end{array}$ & $\begin{array}{c}-0.00780^{*} \\
(0.00427)\end{array}$ & $\begin{array}{l}0.147^{* * *} \\
(0.0173)\end{array}$ & $\begin{array}{c}0.0581^{* * *} \\
(0.0101)\end{array}$ & $\begin{array}{l}0.0232^{* * *} \\
(0.00577)\end{array}$ & $\begin{array}{c}0.00314 \\
(0.00517)\end{array}$ & $\begin{array}{l}0.0161^{* * *} \\
(0.00240)\end{array}$ & $\begin{array}{c}0.206^{* * *} \\
(0.00965)\end{array}$ & $\begin{array}{l}0.0396^{* * *} \\
(0.00352)\end{array}$ & $\begin{array}{c}-0.0309^{* * *} \\
(0.00695)\end{array}$ & $\begin{array}{c}-0.228^{* * *} \\
(0.0150)\end{array}$ \\
\hline Married & $\begin{array}{l}-0.00685 \\
(0.00748)\end{array}$ & $\begin{array}{c}0.0129^{*} \\
(0.00710)\end{array}$ & $\begin{array}{c}0.0157^{*} \\
(0.00956)\end{array}$ & $\begin{array}{c}0.00210 \\
(0.00290)\end{array}$ & $\begin{array}{c}-0.00906 \\
(0.00753)\end{array}$ & $\begin{array}{c}-0.00216 \\
(0.00304)\end{array}$ & $\begin{array}{l}-0.00653 \\
(0.00807)\end{array}$ & $\begin{array}{l}-0.00455 \\
(0.00305)\end{array}$ & $\begin{array}{c}0.00738^{* * *} \\
(0.00260)\end{array}$ & $\begin{array}{c}1.30 \mathrm{e}-05 \\
(0.00326)\end{array}$ & $\begin{array}{l}-8.62 \mathrm{e}-05 \\
(0.00146)\end{array}$ & $\begin{array}{c}-0.00849^{* *} \\
(0.00387)\end{array}$ & $\begin{array}{l}-0.00242 \\
(0.00240)\end{array}$ & $\begin{array}{c}-0.0357^{* * *} \\
(0.00688)\end{array}$ & $\begin{array}{c}0.00412 \\
(0.00675)\end{array}$ \\
\hline Reg_Partnership & $\begin{array}{r}-0.00869 \\
(0.0183)\end{array}$ & $\begin{array}{c}0.0150 \\
(0.0153)\end{array}$ & $\begin{array}{c}-0.0160 \\
(0.0149)\end{array}$ & $\begin{array}{c}0.00155 \\
(0.00449)\end{array}$ & $\begin{array}{r}-0.00810 \\
(0.0138)\end{array}$ & $\begin{array}{c}0.00477 \\
(0.00767)\end{array}$ & $\begin{array}{c}-0.00748 \\
(0.0147)\end{array}$ & $\begin{array}{c}-0.00514 \\
(0.00564)\end{array}$ & $\begin{array}{l}-0.00180 \\
(0.00750)\end{array}$ & $\begin{array}{c}0.00182 \\
(0.00707)\end{array}$ & $\begin{array}{c}0.00293 \\
(0.00285)\end{array}$ & $\begin{array}{c}-0.00257 \\
(0.00857)\end{array}$ & $\begin{array}{r}-0.000811 \\
(0.00403)\end{array}$ & $\begin{array}{l}344^{* * *} \\
0784)\end{array}$ & $\begin{array}{l}0.00956 \\
(0.0149)\end{array}$ \\
\hline Separated & $\begin{array}{l}0.00882 \\
(0.0179)\end{array}$ & $\begin{array}{l}0.00868 \\
(0.0123)\end{array}$ & $\begin{array}{c}0.0135 \\
(0.0171)\end{array}$ & $\begin{array}{l}0.0188^{* * *} \\
(0.00607)\end{array}$ & & & $\begin{array}{c}0.00625 \\
(0.00981)\end{array}$ & & $\begin{array}{c}0.0121 \\
(0.00827)\end{array}$ & & $\begin{array}{l}332 \\
330)\end{array}$ & $\begin{array}{l}282 \\
7773)\end{array}$ & & $\begin{array}{l}4^{* * *} \\
18)\end{array}$ & $\begin{array}{l}150 \\
196)\end{array}$ \\
\hline Divorced & $\begin{array}{c}0.0334^{\star * *} \\
(0.0111)\end{array}$ & $\begin{array}{l}0.0236^{* * *} \\
(0.00847)\end{array}$ & & & & & & & & & & & & $\begin{array}{l}-0.00799 \\
(0.00672)\end{array}$ & $\begin{array}{c}-0.0220^{* * *} \\
(0.00792)\end{array}$ \\
\hline Widowed & $\begin{array}{c}0.00440 \\
(0.00930)\end{array}$ & & & & & & & $\begin{array}{l}0.00706^{* *} \\
(0.00355)\end{array}$ & $\begin{array}{l}0.000655 \\
(0.00242)\end{array}$ & & $\begin{array}{l}-0.000652 \\
(0.00184)\end{array}$ & & $\begin{array}{l}0.00576^{* *} \\
(0.00253)\end{array}$ & $\begin{array}{l}-0.0394^{* * *} \\
(0.00545)\end{array}$ & $\begin{array}{c}-0.0145^{\star} \\
(0.00829)\end{array}$ \\
\hline Retired & & & & & & & & & & & & & & $\begin{array}{c}-0.0892^{* * *} \\
(0.00536)\end{array}$ & $\begin{array}{l}0.0773^{* * *} \\
(0.00806)\end{array}$ \\
\hline nployed & & & & & & & & & & & & & & & \\
\hline $\mathrm{H}$ & $\begin{array}{l}-0.10 \\
(0.0\end{array}$ & & & $1^{* * *}$ & & & & & & & & $\begin{array}{c}-0.0141^{* \star *} \\
(0.00369)\end{array}$ & $\begin{array}{c}-0.0127^{\star \star *} \\
(0.00227)\end{array}$ & $\begin{array}{l}-0.101^{* * *} \\
(0.00718)\end{array}$ & $\begin{array}{c}0.0934^{* * *} \\
(0.0101)\end{array}$ \\
\hline Other_Jok & $\begin{array}{c}-0.166^{\star * \star} \\
(0.0292)\end{array}$ & $\begin{array}{c}-0.0276^{* *} \\
(0.0113)\end{array}$ & $\begin{array}{l}-0.0323^{*} \\
(0.0173)\end{array}$ & $\begin{array}{c}-0.0189^{* \star *} \\
(0.00619)\end{array}$ & $\begin{array}{c}-0.0262^{* *} \\
(0.0121)\end{array}$ & $\begin{array}{c}-0.0217^{* \star *} \\
(0.00723)\end{array}$ & $\begin{array}{c}-0.0540^{* * *} \\
(0.0123)\end{array}$ & $\begin{array}{l}-0.0210^{\star * *} \\
(0.00670)\end{array}$ & $\begin{array}{c}-0.0128 \\
(0.00913)\end{array}$ & $\begin{array}{l}-0.0272^{\star \star *} \\
(0.00686)\end{array}$ & $\begin{array}{c}-0.00505^{\star *} \\
(0.00256)\end{array}$ & & $\begin{array}{l}-0.0110^{* *} \\
(0.00427)\end{array}$ & $\begin{array}{c}-0.0767^{* \star *} \\
(0.0130)\end{array}$ & $\begin{array}{c}0.0883^{* * *} \\
(0.0120)\end{array}$ \\
\hline N_Children & $\begin{array}{l}-0.00347 \\
(0.00219)\end{array}$ & $\begin{array}{l}0.000125 \\
(0.00132)\end{array}$ & $\begin{array}{l}-0.00203 \\
(0.00137)\end{array}$ & & $\begin{array}{c}0.00252^{*} \\
(0.00145)\end{array}$ & $\begin{array}{c}0.000858^{*} \\
(0.000513)\end{array}$ & $\begin{array}{l}0.000903 \\
(0.00211)\end{array}$ & $\begin{array}{c}-0.00173^{*} \\
(0.000886)\end{array}$ & $\begin{array}{l}-0.00239^{* * *} \\
(0.000754)\end{array}$ & $\begin{array}{l}-0.000619 \\
(0.00105)\end{array}$ & $\begin{array}{c}0.000208 \\
(0.000343)\end{array}$ & $\begin{array}{l}-0.00205 \\
(0.00127)\end{array}$ & $\begin{array}{l}-0.000520 \\
(0.000575)\end{array}$ & $\begin{array}{l}-0.00173 \\
(0.00138)\end{array}$ & $\begin{array}{c}0.00458^{* * *} \\
(0.00177)\end{array}$ \\
\hline N_Grandchildren & $\begin{array}{l}0.00451^{* * *} \\
(0.000652)\end{array}$ & $\begin{array}{l}0.00272^{* * *} \\
(0.000641)\end{array}$ & $\begin{array}{l}0.00270^{* * *} \\
(0.000599)\end{array}$ & $\begin{array}{c}0.000328 \\
(0.000449)\end{array}$ & $\begin{array}{l}0.00161^{* * *} \\
(0.000578)\end{array}$ & $0.000625^{* *}$ & $\begin{array}{c}0.00220^{* *} \\
(0.000885)\end{array}$ & $\begin{array}{c}0.000729 \\
(0.000454)\end{array}$ & $\begin{array}{c}2.46 \mathrm{e}-05 \\
(0.000267)\end{array}$ & $\begin{array}{l}0.000862^{*} \\
(0.000469)\end{array}$ & $\begin{array}{c}1.05 \mathrm{e}-05 \\
(0.000155)\end{array}$ & $\begin{array}{c}0.000542 \\
(0.000486)\end{array}$ & $\begin{array}{c}0.000187 \\
(0.000256)\end{array}$ & $\begin{array}{c}0.000372 \\
(0.000560)\end{array}$ & $\begin{array}{l}-0.00408^{* * *} \\
(0.000667)\end{array}$ \\
\hline Logincome & $\begin{array}{l}0.00541^{\text {** }} \\
(0.00268)\end{array}$ & $\begin{array}{l}0.000910 \\
(0.00116)\end{array}$ & $\begin{array}{l}-0.00134 \\
(0.00188)\end{array}$ & $\begin{array}{c}0.00125^{*} \\
(0.000656)\end{array}$ & $\begin{array}{l}-0.000195 \\
(0.00119)\end{array}$ & $\begin{array}{l}0.00227^{* *} \\
(0.00108)\end{array}$ & $\begin{array}{c}0.00149 \\
(0.00141)\end{array}$ & $\begin{array}{l}0.000495 \\
(0.00175)\end{array}$ & $\begin{array}{l}0.00406^{* * *} \\
(0.00102)\end{array}$ & $\begin{array}{l}4.50 \mathrm{e}-05 \\
(0.00102)\end{array}$ & $\begin{array}{l}0.000346^{*} \\
(0.000181)\end{array}$ & $\begin{array}{c}0.00234 \\
(0.00156)\end{array}$ & $\begin{array}{c}0.000715 \\
(0.000512)\end{array}$ & $\begin{array}{c}0.00628^{\star * \star} \\
(0.00158)\end{array}$ & $\begin{array}{c}-0.00667^{* * *} \\
(0.00231)\end{array}$ \\
\hline $\begin{array}{l}\text { Year dummies } \\
\text { Country dummies }\end{array}$ & $\begin{array}{l}\text { YES } \\
\text { YES }\end{array}$ & $\begin{array}{l}\text { YES } \\
\text { YES }\end{array}$ & $\begin{array}{l}\text { YES } \\
\text { YES }\end{array}$ & $\begin{array}{l}\text { YES } \\
\text { YES }\end{array}$ & YES & YES & $\begin{array}{l}\text { YES } \\
\text { YES }\end{array}$ & $\begin{array}{l}\text { YES } \\
\text { YES }\end{array}$ & $\begin{array}{l}\text { YES } \\
\text { YES }\end{array}$ & YES & YES & YE & YE & YES & YES \\
\hline $\begin{array}{l}\text { Observations } \\
\text { N. respondents }\end{array}$ & $\begin{array}{c}103,412 \\
69,573\end{array}$ & 69,533 & $\begin{array}{c}103,369 \\
69,533\end{array}$ & $\begin{array}{c}103,369 \\
69,533\end{array}$ & $\begin{array}{c}103,369 \\
69,533\end{array}$ & 69,533 & $\begin{array}{c}103,369 \\
69,533\end{array}$ & $\begin{array}{c}103,369 \\
69,533\end{array}$ & 69,533 & $\begin{array}{c}103,369 \\
69,533\end{array}$ & $\begin{array}{c}103,369 \\
69,533\end{array}$ & $\begin{array}{c}103,369 \\
69,533\end{array}$ & $\begin{array}{c}103,369 \\
69,533\end{array}$ & $\begin{array}{c}103,369 \\
69,533\end{array}$ & $\begin{array}{c}103,369 \\
69,533\end{array}$ \\
\hline
\end{tabular}

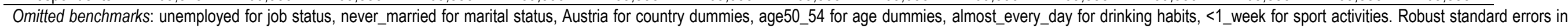
parentheses clustered at country level. ${ }^{* * *} p<0.01,{ }^{* *} p<0.05,{ }^{*} p<0.1$. 
Table A4c - Education years and specific diseases (attrition-adjusted)

\begin{tabular}{|c|c|c|c|c|c|c|c|c|c|c|c|c|c|c|c|}
\hline VARIABLES & $\begin{array}{c}1) \\
\text { longterm } \\
\text { illness }\end{array}$ & $\begin{array}{c}(2) \\
\text { heart } \\
\text { attack }\end{array}$ & $\begin{array}{c}(3) \\
\text { hyper } \\
\text { tension }\end{array}$ & stroke & $\begin{array}{c}(5) \\
\text { diabetes }\end{array}$ & asthma & arthritis & $\begin{array}{c}(8) \\
\text { osteopor }\end{array}$ & $\begin{array}{c}\text { (9) } \\
\text { cancer }\end{array}$ & $\begin{array}{l}\text { (10) } \\
\text { ulcer }\end{array}$ & parkinson & (12) & $\begin{array}{c}\text { (13) } \\
\text { femoralfra. }\end{array}$ & $\begin{array}{c}\text { (14) } \\
\text { othercond. }\end{array}$ & nocondi. \\
\hline Eduyears & $\begin{array}{c}-0.00648^{* * *} \\
(0.00141)\end{array}$ & $\begin{array}{c}-0.00333^{\star * \star} \\
(0.000413)\end{array}$ & $\begin{array}{c}-0.00589^{* \star} \\
(0.00207)\end{array}$ & $\begin{array}{c}-0.00145^{\star * *} \\
(0.000230)\end{array}$ & $\begin{array}{c}-0.00452^{* * \star} \\
(0.000467)\end{array}$ & $\begin{array}{c}-0.00197^{*} \\
(0.000968)\end{array}$ & $\begin{array}{c}-0.00500^{* *} \\
(0.00176)\end{array}$ & $\begin{array}{c}-0.00157^{* * *} \\
(0.000363)\end{array}$ & $\begin{array}{c}0.00103^{*} \\
(0.000518)\end{array}$ & $\begin{array}{c}-0.00141^{\text {** }} \\
(0.000429)\end{array}$ & $\begin{array}{c}-0.000166 \\
(0.000110)\end{array}$ & $\begin{array}{c}-0.000566 \\
(0.00116)\end{array}$ & $\begin{array}{l}-0.000450^{*} \\
(0.000213)\end{array}$ & $\begin{array}{c}-0.00139 \\
(0.00108)\end{array}$ & $\begin{array}{l}0.00539^{* *} \\
(0.00186)\end{array}$ \\
\hline Female & $\begin{array}{l}0.0316^{\star \star \star} \\
(0.00797)\end{array}$ & $\begin{array}{c}-0.0717^{\star * \star} \\
(0.0118)\end{array}$ & $\begin{array}{c}0.0187^{* *} \\
(0.00679)\end{array}$ & $\begin{array}{c}-0.0139^{\star \star \star} \\
(0.00431)\end{array}$ & $\begin{array}{c}-0.0252^{\star \star \star *} \\
(0.00489)\end{array}$ & $\begin{array}{l}0.00975^{*} \\
(0.00500)\end{array}$ & $\begin{array}{c}0.0954^{* \star *} \\
(0.0164)\end{array}$ & $\begin{array}{c}0.0823^{\star * \star} \\
(0.0171)\end{array}$ & $\begin{array}{l}0.0181^{* * *} \\
(0.00574)\end{array}$ & $\begin{array}{c}-0.0167^{* * *} \\
(0.00220)\end{array}$ & $\begin{array}{l}-0.00132 \\
(0.00137)\end{array}$ & $\begin{array}{l}0.0312^{* * *} \\
(0.00943)\end{array}$ & $\begin{array}{l}0.00422^{*} \\
(0.00220)\end{array}$ & $\begin{array}{l}0.0428^{*} \\
(0.0206)\end{array}$ & $\begin{array}{l}-0.0232^{*} \\
(0.0114)\end{array}$ \\
\hline Age55_59 & $\begin{array}{l}0.0365^{\star \star \star} \\
(0.00644)\end{array}$ & $\begin{array}{c}0.00610 \\
(0.00517)\end{array}$ & $\begin{array}{l}0.0744^{\star \star \star} \\
(0.00810)\end{array}$ & $\begin{array}{l}0.00608^{* *} \\
(0.00258)\end{array}$ & $\begin{array}{c}0.00806 \\
(0.00522)\end{array}$ & $\begin{array}{l}-0.00180 \\
(0.00637)\end{array}$ & $\begin{array}{l}0.0481^{* \star *} \\
(0.00432)\end{array}$ & $\begin{array}{l}0.0227^{\star \star \star} \\
(0.00581)\end{array}$ & $\begin{array}{c}0.0113^{*} \\
(0.00584)\end{array}$ & $\begin{array}{c}0.00449 \\
(0.00290)\end{array}$ & $\begin{array}{l}-0.000260 \\
(0.000734)\end{array}$ & $\begin{array}{c}0.00401 \\
(0.00365)\end{array}$ & $\begin{array}{c}0.00382 \\
(0.00381)\end{array}$ & $\begin{array}{c}0.00578 \\
(0.00904)\end{array}$ & $\begin{array}{l}-0.108^{\star * \star} \\
(0.00832)\end{array}$ \\
\hline Age60_64 & $\begin{array}{l}0.0326^{\star \star \star} \\
(0.00913)\end{array}$ & $\begin{array}{c}0.0151 \\
(0.00933)\end{array}$ & $\begin{array}{l}0.111^{\star \star *} \\
(0.0141)\end{array}$ & $\begin{array}{c}0.00259 \\
(0.00297)\end{array}$ & $\begin{array}{l}0.0170^{\star \star \star} \\
(0.00509)\end{array}$ & $\begin{array}{c}-0.00312 \\
(0.00738)\end{array}$ & $\begin{array}{c}0.0742^{\star \star \star} \\
(0.0156)\end{array}$ & $\begin{array}{c}0.0365^{\star \star \star} \\
(0.0105)\end{array}$ & $\begin{array}{l}0.0131^{* \star \star} \\
(0.00350)\end{array}$ & $\begin{array}{c}0.00622 \\
(0.00398)\end{array}$ & $\begin{array}{c}0.00161 \\
(0.00142)\end{array}$ & $\begin{array}{l}0.0219^{* * *} \\
(0.00589)\end{array}$ & $\begin{array}{l}0.000328 \\
(0.00180)\end{array}$ & $\begin{array}{c}-0.0360^{* \star *} \\
(0.00706)\end{array}$ & $\begin{array}{c}-0.139^{\star * \star *} \\
(0.0128)\end{array}$ \\
\hline Age65_69 & $\begin{array}{c}0.0295 \\
(0.0282)\end{array}$ & $\begin{array}{l}0.0333^{\star \star \star} \\
(0.00553)\end{array}$ & $\begin{array}{l}0.168^{\star \star \star} \\
(0.0184)\end{array}$ & $\begin{array}{c}0.00718 \\
(0.00504)\end{array}$ & $\begin{array}{c}0.0321^{\star \star \star} \\
(0.0100)\end{array}$ & $\begin{array}{c}-0.0112^{*} \\
(0.00527)\end{array}$ & $\begin{array}{l}0.0699^{* \star} \\
(0.0256)\end{array}$ & $\begin{array}{c}0.0518^{\star \star \star} \\
(0.0117)\end{array}$ & $\begin{array}{c}0.0184^{* *} \\
(0.00786)\end{array}$ & $\begin{array}{l}-0.00178 \\
(0.00485)\end{array}$ & $\begin{array}{c}0.00140 \\
(0.00255)\end{array}$ & $\begin{array}{l}0.0556^{\star \star \star} \\
(0.00726)\end{array}$ & $\begin{array}{l}9.95 \mathrm{e}-05 \\
(0.00338)\end{array}$ & $\begin{array}{c}-0.0337^{\star \star *} \\
(0.00668)\end{array}$ & $\begin{array}{c}-0.156^{\star \star \star} \\
(0.0179)\end{array}$ \\
\hline Age70_74 & $\begin{array}{l}0.0812^{* *} \\
(0.0275)\end{array}$ & $\begin{array}{l}0.0801^{* * \star} \\
(0.00493)\end{array}$ & $\begin{array}{l}0.202^{* \star \star} \\
(0.0186)\end{array}$ & $\begin{array}{c}0.0182^{* *} \\
(0.00763)\end{array}$ & $\begin{array}{l}0.0333^{* \star *} \\
(0.00854)\end{array}$ & $\begin{array}{c}-0.0108 \\
(0.00675)\end{array}$ & $\begin{array}{c}0.0822^{* \star *} \\
(0.0198)\end{array}$ & $\begin{array}{c}0.0680^{\star \star \star} \\
(0.0172)\end{array}$ & $\begin{array}{l}0.0276^{\star \star \star} \\
(0.00836)\end{array}$ & $\begin{array}{l}0.000628 \\
(0.00468)\end{array}$ & $\begin{array}{c}0.00311 \\
(0.00320)\end{array}$ & $\begin{array}{c}0.0858^{* * *} \\
(0.0118)\end{array}$ & $\begin{array}{l}0.0128^{\star * \star} \\
(0.00381)\end{array}$ & $\begin{array}{l}-0.0280 \\
(0.0159)\end{array}$ & $\begin{array}{c}-0.220^{\star \star \star} \\
(0.0151)\end{array}$ \\
\hline Age75_79 & $\begin{array}{l}0.110^{* *} \\
(0.0431)\end{array}$ & $\begin{array}{l}0.108^{* * *} \\
(0.0105)\end{array}$ & $\begin{array}{l}0.194^{\star * \star} \\
(0.0191)\end{array}$ & $\begin{array}{l}0.0426^{* \star *} \\
(0.00550)\end{array}$ & $\begin{array}{c}0.0316 \\
(0.0180)\end{array}$ & $\begin{array}{c}-0.00701 \\
(0.00888)\end{array}$ & $\begin{array}{l}0.110^{\star * \star} \\
(0.0276)\end{array}$ & $\begin{array}{c}0.0763^{* \star *} \\
(0.0124)\end{array}$ & $\begin{array}{c}0.0536^{\star * \star} \\
(0.0156)\end{array}$ & $\begin{array}{c}0.0155^{* *} \\
(0.00676)\end{array}$ & $\begin{array}{l}0.00 \\
(0.0\end{array}$ & $\begin{array}{l}0.1 \\
(0.0\end{array}$ & $\begin{array}{l}7^{* \star *} \\
398)\end{array}$ & $\begin{array}{l}613 \\
80)\end{array}$ & $\begin{array}{c}-0.243^{\star * *} \\
(0.0190)\end{array}$ \\
\hline AgeAbove_80 & $\begin{array}{l}0.127^{\star \star \star} \\
(0.0342)\end{array}$ & $\begin{array}{l}0.138^{\star \star \star} \\
(0.0113)\end{array}$ & $\begin{array}{l}0.156^{\star \star \star} \\
(0.0181)\end{array}$ & $\begin{array}{l}0.0510^{\star \star \star} \\
(0.00348)\end{array}$ & $\begin{array}{c}0.0370^{* * \star} \\
(0.0120)\end{array}$ & $\begin{array}{c}-0.00836 \\
(0.00998)\end{array}$ & $\begin{array}{l}0.114^{* \star *} \\
(0.0275)\end{array}$ & $\begin{array}{c}0.0932^{* \star \star} \\
(0.0117)\end{array}$ & $\begin{array}{c}0.0544^{* * *} \\
(0.0124)\end{array}$ & $\begin{array}{c}0.00910 \\
(0.00759)\end{array}$ & $\begin{array}{r}0.0 \\
(0.00\end{array}$ & $\begin{array}{l}0.253^{* \star \star} \\
(0.0305)\end{array}$ & $\begin{array}{l}4^{* \star \star} \\
198)\end{array}$ & $\begin{array}{l}-0.0181^{* *} \\
(0.00749)\end{array}$ & $\begin{array}{c}-0.262^{* \star \star} \\
(0.0167)\end{array}$ \\
\hline Married & $\begin{array}{c}-0.0353^{* \star} \\
(0.0161)\end{array}$ & $\begin{array}{c}0.00400 \\
(0.00504)\end{array}$ & $\begin{array}{l}0.00662 \\
(0.0203)\end{array}$ & $\begin{array}{c}0.00108 \\
(0.00876)\end{array}$ & $\begin{array}{l}-0.0166^{* *} \\
(0.00588)\end{array}$ & $\begin{array}{c}0.00567 \\
(0.00753)\end{array}$ & $\begin{array}{c}0.0121 \\
(0.0141)\end{array}$ & $\begin{array}{c}-0.0152^{*} \\
(0.00737)\end{array}$ & $\begin{array}{l}0.00311 \\
(0.0103)\end{array}$ & $\begin{array}{l}-0.00265 \\
(0.0109)\end{array}$ & $\begin{array}{c}0.00188 \\
(0.00250)\end{array}$ & $\begin{array}{c}-0.00930 \\
(0.00861)\end{array}$ & & $\begin{array}{c}-0.0607^{* * *} \\
(0.0155)\end{array}$ & $\begin{array}{l}0.00500 \\
(0.0221)\end{array}$ \\
\hline $\mathrm{Re}$ & $\begin{array}{l}-0.0347 \\
(0.0232)\end{array}$ & $\begin{array}{c}-0.0435^{\star \star *} \\
(0.0129)\end{array}$ & $\begin{array}{c}-0.0693^{* * *} \\
(0.0197)\end{array}$ & $\begin{array}{l}-0.0167 \\
(0.0117)\end{array}$ & $\begin{array}{l}-0.0291^{* * *} \\
(0.00734)\end{array}$ & $\begin{array}{c}0.00286 \\
(0.00914)\end{array}$ & $\begin{array}{c}0.0110 \\
(0.0176)\end{array}$ & $\begin{array}{c}-0.0175^{\star} \\
(0.00934)\end{array}$ & $\begin{array}{c}0.0173 \\
(0.0140)\end{array}$ & $\begin{array}{l}0.00170 \\
(0.0104)\end{array}$ & $\begin{array}{l}0.00842^{\star * *} \\
(0.00254)\end{array}$ & $\begin{array}{l}-0.0135 \\
(0.0250)\end{array}$ & $\begin{array}{l}-0.0112^{*} \\
(0.00531)\end{array}$ & $\begin{array}{c}-0.0756^{* * *} \\
(0.0158)\end{array}$ & $\begin{array}{l}0.0377^{*} \\
(0.0196)\end{array}$ \\
\hline Separated & $\begin{array}{c}0.000402 \\
(0.0352)\end{array}$ & $\begin{array}{c}-0.00766 \\
(0.0215)\end{array}$ & $\begin{array}{l}-0.0200 \\
(0.0369)\end{array}$ & $\begin{array}{c}0.0258 \\
(0.0258)\end{array}$ & $\begin{array}{c}0.0518 \\
(0.0313)\end{array}$ & $\begin{array}{c}-0.00137 \\
(0.0145)\end{array}$ & $\begin{array}{l}0.0733^{*} \\
(0.0404)\end{array}$ & $\begin{array}{l}-0.0242 \\
(0.0178)\end{array}$ & $\begin{array}{l}0.00329 \\
(0.0280)\end{array}$ & $\begin{array}{l}0.0706^{*} \\
(0.0376)\end{array}$ & & $\begin{array}{c}0.0204 \\
(0.0283)\end{array}$ & $\begin{array}{c}0.00108 \\
(0.00919)\end{array}$ & & $\begin{array}{c}-0.0555^{* *} \\
(0.0208)\end{array}$ \\
\hline Divorced & $\begin{array}{l}0.0405^{* *} \\
(0.0177)\end{array}$ & $\begin{array}{l}0.0396^{*} \\
(0.0207)\end{array}$ & $\begin{array}{l}0.00478 \\
(0.0248)\end{array}$ & $\begin{array}{l}0.00856 \\
(0.0127)\end{array}$ & $\begin{array}{c}0.00877 \\
(0.00903)\end{array}$ & $\begin{array}{c}0.0200^{*} \\
(0.00997)\end{array}$ & $\begin{array}{l}0.0577^{* \star} \\
(0.0192)\end{array}$ & $\begin{array}{l}0.00218 \\
(0.0128)\end{array}$ & $\begin{array}{c}0.0189 \\
(0.0191)\end{array}$ & $\begin{array}{c}0.00625 \\
(0.00793)\end{array}$ & $\begin{array}{c}0.00138 \\
(0.00208)\end{array}$ & $\begin{array}{c}-0.000611 \\
(0.0103)\end{array}$ & $\begin{array}{l}0.00962 \\
(0.0108)\end{array}$ & $\begin{array}{l}-0.0293^{*} \\
(0.0145)\end{array}$ & $\begin{array}{l}-0.0395 \\
(0.0231)\end{array}$ \\
\hline Widowed & $\begin{array}{l}-0.0266 \\
(0.0176)\end{array}$ & $\begin{array}{r}-0.000605 \\
(0.00958)\end{array}$ & $\begin{array}{c}0.0430 \\
(0.0352)\end{array}$ & $\begin{array}{c}0.00234 \\
(0.00962)\end{array}$ & $\begin{array}{c}0.0142^{*} \\
(0.00734)\end{array}$ & $\begin{array}{l}0.00546 \\
(0.0142)\end{array}$ & $\begin{array}{c}0.0173 \\
(0.0152)\end{array}$ & $\begin{array}{l}-0.0146 \\
(0.0130)\end{array}$ & $\begin{array}{l}-0.00259 \\
(0.0106)\end{array}$ & $\begin{array}{c}0.00372 \\
(0.00882)\end{array}$ & $\begin{array}{l}0.00485^{\star} \\
(0.00227)\end{array}$ & $\begin{array}{c}0.0142 \\
(0.0164)\end{array}$ & $\begin{array}{c}0.0128^{*} \\
(0.00667)\end{array}$ & $\begin{array}{c}-0.0640^{\star * *} \\
(0.0205)\end{array}$ & $\begin{array}{l}-0.0142 \\
(0.0295)\end{array}$ \\
\hline Retirec & $\begin{array}{c}-0.191^{* \star *} \\
(0.0242)\end{array}$ & $\begin{array}{c}-0.0277^{\star *} \\
(0.0112)\end{array}$ & $\begin{array}{c}-0.0139 \\
(0.0154)\end{array}$ & $\begin{array}{l}-0.0330^{*} \\
(0.0164)\end{array}$ & $\begin{array}{c}-0.0277^{* \star} \\
(0.0115)\end{array}$ & $\begin{array}{c}-0.0119 \\
(0.00933)\end{array}$ & $\begin{array}{l}-0.0660^{\star} \\
(0.0309)\end{array}$ & $\begin{array}{c}-0.0298^{* * *} \\
(0.00738)\end{array}$ & $\begin{array}{l}-0.0206^{* *} \\
(0.00763)\end{array}$ & $\begin{array}{c}-0.00748 \\
(0.00708)\end{array}$ & $\begin{array}{l}-0.00170 \\
(0.00434)\end{array}$ & $\begin{array}{c}-0.000294 \\
(0.0111)\end{array}$ & $\begin{array}{l}-0.00867 \\
(0.00492)\end{array}$ & $\begin{array}{c}-0.0742^{\star * \star} \\
(0.0139)\end{array}$ & $\begin{array}{c}0.0998^{* \star *} \\
(0.0173)\end{array}$ \\
\hline Empl & $\begin{array}{r}-0.323^{* \star *} \\
(0.0214)\end{array}$ & $\begin{array}{c}-0.0769^{* * *} \\
(0.00713)\end{array}$ & $\begin{array}{c}-0.0472^{\star * *} \\
(0.0109)\end{array}$ & $\begin{array}{c}-0.0574^{\star * \star} \\
(0.0112)\end{array}$ & $\begin{array}{c}-0.0534^{\star * \star} \\
(0.00964)\end{array}$ & $\begin{array}{l}-0.0203^{*} \\
(0.0114)\end{array}$ & $\begin{array}{c}-0.109^{* \star *} \\
(0.0161)\end{array}$ & $\begin{array}{c}-0.0337^{* * *} \\
(0.00588)\end{array}$ & $\begin{array}{c}-0.0452^{* * *} \\
(0.00585)\end{array}$ & $\begin{array}{l}-0.0226^{* *} \\
(0.00950)\end{array}$ & $\begin{array}{l}-0.00573^{*} \\
(0.00321)\end{array}$ & $\begin{array}{c}-0.0139 \\
(0.0108)\end{array}$ & $\begin{array}{c}-0.0147^{* * *} \\
(0.00272)\end{array}$ & $\begin{array}{c}-0.124^{* * \star} \\
(0.0114)\end{array}$ & $\begin{array}{l}0.204^{\star * \star} \\
(0.0168)\end{array}$ \\
\hline Hom & $\begin{array}{c}-0.224^{* \star *} \\
(0.0182)\end{array}$ & $\begin{array}{c}-0.0314^{*} \\
(0.0162)\end{array}$ & $\begin{array}{r}-0.00101 \\
(0.0113)\end{array}$ & $\begin{array}{c}-0.0476^{* *} \\
(0.0174)\end{array}$ & $\begin{array}{c}-0.0272^{* *} \\
(0.0101)\end{array}$ & $\begin{array}{c}-0.0265^{\star \star \star} \\
(0.00764)\end{array}$ & $\begin{array}{c}-0.0770^{* *} \\
(0.0261)\end{array}$ & $\begin{array}{l}-0.0165 \\
(0.0153)\end{array}$ & $\begin{array}{c}-0.0311^{* * *} \\
(0.0100)\end{array}$ & $\begin{array}{l}-0.0195^{* \star} \\
(0.00767)\end{array}$ & $\begin{array}{l}-0.0 \\
(0.0\end{array}$ & & $\begin{array}{c}-0.00860^{* *} \\
(0.00385)\end{array}$ & $\begin{array}{c}-0.106^{\star \star \star} \\
(0.0192)\end{array}$ & $\begin{array}{l}0.119^{\star \star \star} \\
(0.0155)\end{array}$ \\
\hline Other_Job & $\begin{array}{l}-0.175^{\star \star *} \\
(0.0295)\end{array}$ & $\begin{array}{l}-0.0489^{*} \\
(0.0245)\end{array}$ & $\begin{array}{l}-0.0626 \\
(0.0371)\end{array}$ & $\begin{array}{l}-0.0161^{*} \\
(0.00760)\end{array}$ & $\begin{array}{l}-0.0261 \\
(0.0184)\end{array}$ & $\begin{array}{c}-0.0380^{* \star} \\
(0.0154)\end{array}$ & $\begin{array}{c}-0.0640^{* *} \\
(0.0294)\end{array}$ & $\begin{array}{c}0.0104 \\
(0.0156)\end{array}$ & $\begin{array}{l}-0.00643 \\
(0.0202)\end{array}$ & $\begin{array}{l}-0.00837 \\
(0.00929)\end{array}$ & $\begin{array}{c}-0.00630 \\
(0.00508)\end{array}$ & $\begin{array}{l}-0.0140 \\
(0.0197)\end{array}$ & & & $\begin{array}{c}0.0467 \\
(0.0329)\end{array}$ \\
\hline N_Children & $\begin{array}{l}-0.00726^{*} \\
(0.00341)\end{array}$ & $\begin{array}{c}0.00194 \\
(0.00184)\end{array}$ & $\begin{array}{c}0.00206 \\
(0.00160)\end{array}$ & $\begin{array}{l}0.00312^{* *} \\
(0.00136)\end{array}$ & $\begin{array}{c}0.00305 \\
(0.00352)\end{array}$ & $\begin{array}{c}0.00102 \\
(0.00176)\end{array}$ & $\begin{array}{l}0.00368^{*} \\
(0.00203)\end{array}$ & $\begin{array}{l}-0.00210^{* *} \\
(0.000731)\end{array}$ & $\begin{array}{c}-0.00221 \\
(0.00178)\end{array}$ & $\begin{array}{c}-0.00145 \\
(0.000868)\end{array}$ & $\begin{array}{c}-1.94 \mathrm{e}-05 \\
(0.000647)\end{array}$ & $\begin{array}{l}-0.00312 \\
(0.00226)\end{array}$ & $\begin{array}{c}-0.00217 \\
(0.00190)\end{array}$ & $\begin{array}{c}-0.00382^{*} \\
(0.00210)\end{array}$ & $\begin{array}{l}0.00802^{* *} \\
(0.00353)\end{array}$ \\
\hline N & $\begin{array}{c}0.00628^{* * *} \\
(0.00180)\end{array}$ & $\begin{array}{l}0.00334^{\star *} \\
(0.00130)\end{array}$ & $\begin{array}{c}0.00183 \\
(0.00134)\end{array}$ & $\begin{array}{c}-0.000902^{*} \\
(0.000468)\end{array}$ & $\begin{array}{l}0.00255^{\star \star \star} \\
(0.000738)\end{array}$ & $\begin{array}{c}0.00141^{* *} \\
(0.000612)\end{array}$ & $\begin{array}{l}0.00233^{*} \\
(0.00113)\end{array}$ & $\begin{array}{l}0.000624 \\
(0.00121)\end{array}$ & $\begin{array}{c}0.000437 \\
(0.000831)\end{array}$ & $\begin{array}{c}0.00122 \\
(0.000803)\end{array}$ & $\begin{array}{l}-0.000127 \\
(0.000535)\end{array}$ & $\begin{array}{l}0.000213 \\
(0.00150)\end{array}$ & $\begin{array}{c}0.000379 \\
(0.000649)\end{array}$ & $\begin{array}{c}0.000318 \\
(0.000955)\end{array}$ & $\begin{array}{c}-0.00571^{\star \star \star *} \\
(0.00148)\end{array}$ \\
\hline Loginc & $\begin{array}{c}0.0178^{*} \\
(0.00839)\end{array}$ & $\begin{array}{l}0.00574^{\star *} \\
(0.00214)\end{array}$ & $\begin{array}{c}0.00942 \\
(0.00607)\end{array}$ & $\begin{array}{l}0.00228^{* * \star} \\
(0.000534)\end{array}$ & $\begin{array}{l}0.00371^{*} \\
(0.00203)\end{array}$ & $\begin{array}{c}0.000695 \\
(0.000462)\end{array}$ & $\begin{array}{l}0.00948^{* *} \\
(0.00428)\end{array}$ & $\begin{array}{c}0.00113 \\
(0.00108)\end{array}$ & $\begin{array}{l}0.00280^{\star * \star} \\
(0.000757)\end{array}$ & $\begin{array}{c}0.00110 \\
(0.000786)\end{array}$ & $\begin{array}{l}0.000564^{* *} \\
(0.000208)\end{array}$ & $\begin{array}{c}0.00744^{* * *} \\
(0.00222)\end{array}$ & $\begin{array}{l}0.00179^{\star * \star} \\
(0.000483)\end{array}$ & $\begin{array}{c}0.00608^{* \star *} \\
(0.00166)\end{array}$ & $\begin{array}{l}-0.0213^{\star *} \\
(0.00836)\end{array}$ \\
\hline & YES & YES & YES & YES & YES & YES & YES & YES & YES & YE & $Y$ & YE & YE & YE & YES \\
\hline & & YES & YES & $\mathrm{r}$ & YES & & YES & YE & YES & YE & YE & YE & YES & YES & YES \\
\hline $\mathrm{Ob}$ & 85,379 & 85,400 & 85,400 & 85,400 & 85,400 & 85,400 & 85,400 & 85,400 & 85,400 & 85,400 & 85 & 85,400 & 85,400 & 85,400 & 85,400 \\
\hline R-squared & 0.065 & 0.057 & 0.039 & 0.020 & 0.024 & 0.013 & 0.095 & 0.064 & 0.014 & 0.009 & 0.006 & 0.093 & 0.015 & 0.032 & 0.071 \\
\hline
\end{tabular}

Omitted benchmarks: unemployed for job status, never_married for marital status, Austria for country dummies, age50_54 for age dummies, Robust standard errors in parentheses clustered at country level. ${ }^{* \star}$ p $<0.01$, ${ }^{* *} p<0.05,{ }^{*} p<0.1$. 
Table A4d - Education years and specific diseases (attrition-adjusted)

\begin{tabular}{|c|c|c|c|c|c|c|c|c|c|c|c|c|c|c|c|}
\hline VARIABLES & $\begin{array}{c}1) \\
\text { longterm } \\
\text { illness } \\
\end{array}$ & $\begin{array}{c}(2) \\
\text { heart } \\
\text { attack }\end{array}$ & $\begin{array}{c}(3) \\
\text { hyper } \\
\text { tension } \\
\end{array}$ & stroke & diabetes & $\begin{array}{c}(6) \\
\text { asthma }\end{array}$ & $\begin{array}{c}(7) \\
\text { arthritis } \\
\end{array}$ & $\begin{array}{c}(8) \\
\text { osteopor }\end{array}$ & $\begin{array}{c}(9) \\
\text { cancer }\end{array}$ & $\begin{array}{l}(10) \\
\text { ulcer }\end{array}$ & $\begin{array}{c}(11) \\
\text { parkinson }\end{array}$ & $\begin{array}{c}(12) \\
\text { cataracts }\end{array}$ & $\begin{array}{c}\text { (13) } \\
\text { femoralfra. }\end{array}$ & $\begin{array}{c}\text { (14) } \\
\text { othercond. }\end{array}$ & $\begin{array}{c}(15) \\
\text { nocondi. }\end{array}$ \\
\hline Eduyears & $\begin{array}{c}-0.00386^{* *} \\
(0.00135)\end{array}$ & $\begin{array}{c}-0.00245^{\star * *} \\
(0.000486)\end{array}$ & $\begin{array}{c}-0.00334 \\
(0.00217)\end{array}$ & $\begin{array}{c}-0.00101^{* * *} \\
(0.000212)\end{array}$ & $\begin{array}{c}-0.00268^{* * *} \\
(0.000585)\end{array}$ & $\begin{array}{c}-0.00146^{*} \\
(0.000802)\end{array}$ & $\begin{array}{c}-0.00410^{* *} \\
(0.00188)\end{array}$ & $\begin{array}{c}-0.00145^{* * *} \\
(0.000320)\end{array}$ & $\begin{array}{c}0.000988^{*} \\
(0.000537)\end{array}$ & $\begin{array}{l}-0.00115^{\star *} \\
(0.000486)\end{array}$ & $\begin{array}{c}-0.000105 \\
(0.000113)\end{array}$ & $\begin{array}{r}-0.000475 \\
(0.00130)\end{array}$ & $\begin{array}{c}-0.000392 \\
(0.000280)\end{array}$ & $\begin{array}{c}-0.000818 \\
(0.00112)\end{array}$ & $\begin{array}{c}0.00344 \\
(0.00199)\end{array}$ \\
\hline DRINKING & & & & & & & & & & & & & & & \\
\hline 5or6days_week & $\begin{array}{l}0.00386 \\
(0.0142)\end{array}$ & $\begin{array}{l}-0.0207^{\star} \\
(0.0109)\end{array}$ & $\begin{array}{l}-0.0189 \\
(0.0122)\end{array}$ & $\begin{array}{c}-0.0203^{* * *} \\
(0.00582)\end{array}$ & $\begin{array}{c}-0.0354^{\star * *} \\
(0.00812)\end{array}$ & $\begin{array}{l}-0.00797 \\
(0.00678)\end{array}$ & $\begin{array}{l}-0.0159 \\
(0.0141)\end{array}$ & $\begin{array}{c}-0.0105 \\
(0.00894)\end{array}$ & $\begin{array}{c}0.00158 \\
(0.00511)\end{array}$ & $\begin{array}{l}-0.00156 \\
(0.00772)\end{array}$ & $\begin{array}{l}-0.00427 \\
(0.00254)\end{array}$ & $\begin{array}{l}0.00816^{*} \\
(0.00426)\end{array}$ & $\begin{array}{l}0.000383 \\
(0.00342)\end{array}$ & $\begin{array}{c}0.0246^{\star *} \\
(0.00857)\end{array}$ & $\begin{array}{l}-0.0160 \\
(0.0121)\end{array}$ \\
\hline 3or4days_week & $\begin{array}{c}-0.0323^{\star *} \\
(0.0128)\end{array}$ & $\begin{array}{c}-0.0279^{* * \star} \\
(0.00601)\end{array}$ & $\begin{array}{l}-0.0154 \\
(0.0122)\end{array}$ & $\begin{array}{c}-0.0110 \\
(0.00687)\end{array}$ & $\begin{array}{c}-0.0495^{\star \star *} \\
(0.00978)\end{array}$ & $\begin{array}{c}0.00318 \\
(0.00568)\end{array}$ & $\begin{array}{r}-0.000749 \\
(0.00962)\end{array}$ & $\begin{array}{l}-0.0151^{* *} \\
(0.00568)\end{array}$ & $\begin{array}{c}0.00703 \\
(0.00476)\end{array}$ & $\begin{array}{l}-0.00922 \\
(0.00612)\end{array}$ & $\begin{array}{c}-0.00390^{\star *} \\
(0.00163)\end{array}$ & $\begin{array}{l}0.00633 \\
(0.0116)\end{array}$ & $\begin{array}{c}-0.00661^{* * *} \\
(0.000960)\end{array}$ & $\begin{array}{c}-0.00887 \\
(0.0118)\end{array}$ & $\begin{array}{l}0.0189^{* *} \\
(0.00874)\end{array}$ \\
\hline 1or2_week & $\begin{array}{c}-0.0748^{\star * *} \\
(0.00991)\end{array}$ & $\begin{array}{c}-0.0377^{\star * *} \\
(0.00890)\end{array}$ & $\begin{array}{l}-0.0187 \\
(0.0189)\end{array}$ & $\begin{array}{c}-0.0161^{* * *} \\
(0.00481)\end{array}$ & $\begin{array}{c}-0.0538^{\star \star \star} \\
(0.00863)\end{array}$ & $\begin{array}{c}-0.00657^{\star} \\
(0.00345)\end{array}$ & $\begin{array}{l}-0.00731 \\
(0.00969)\end{array}$ & $\begin{array}{l}-0.00536 \\
(0.00571)\end{array}$ & $\begin{array}{c}0.00311 \\
(0.00261)\end{array}$ & $\begin{array}{l}-0.0121^{* *} \\
(0.00526)\end{array}$ & $\begin{array}{l}-0.00180 \\
(0.00219)\end{array}$ & $\begin{array}{c}-0.00873 \\
(0.00774)\end{array}$ & $\begin{array}{c}-0.00402^{* *} \\
(0.00177)\end{array}$ & $\begin{array}{c}-0.0202 \\
(0.0121)\end{array}$ & $\begin{array}{c}0.0174^{*} \\
(0.00914)\end{array}$ \\
\hline 1or2_month & $\begin{array}{c}-0.0486^{* *} \\
(0.0165)\end{array}$ & $\begin{array}{c}-0.0334^{\star \star *} \\
(0.0107)\end{array}$ & $\begin{array}{c}-0.0293^{* *} \\
(0.0123)\end{array}$ & $\begin{array}{c}-0.0275^{\star * *} \\
(0.00720)\end{array}$ & $\begin{array}{c}-0.0678^{\star \star \star} \\
(0.00972)\end{array}$ & $\begin{array}{l}-0.0158^{* * *} \\
(0.00472)\end{array}$ & $\begin{array}{l}-0.0163 \\
(0.0158)\end{array}$ & $\begin{array}{l}-0.0243^{\star \star} \\
(0.00887)\end{array}$ & $\begin{array}{l}0.00779 \\
(0.0130)\end{array}$ & $\begin{array}{l}-0.00980^{*} \\
(0.00540)\end{array}$ & $\begin{array}{c}-0.00441^{* * *} \\
(0.00145)\end{array}$ & $\begin{array}{c}0.00292 \\
(0.00506)\end{array}$ & $\begin{array}{c}0.00520 \\
(0.00669)\end{array}$ & $\begin{array}{l}0.00234 \\
(0.0109)\end{array}$ & $\begin{array}{l}0.0310^{*} \\
(0.0164)\end{array}$ \\
\hline$<1$ month & $\begin{array}{l}-0.106^{\star \star} \\
(0.0356)\end{array}$ & $\begin{array}{c}-0.0499^{* * *} \\
(0.0105)\end{array}$ & $\begin{array}{c}-0.0365^{* * *} \\
(0.00751)\end{array}$ & $\begin{array}{c}-0.0329^{* * *} \\
(0.00552)\end{array}$ & $\begin{array}{c}-0.0968^{* * *} \\
(0.0104)\end{array}$ & $\begin{array}{l}-0.0177^{\star *} \\
(0.00696)\end{array}$ & $\begin{array}{c}-0.00149 \\
(0.0156)\end{array}$ & $\begin{array}{l}-0.0222^{* *} \\
(0.00928)\end{array}$ & $\begin{array}{l}0.00358 \\
(0.0110)\end{array}$ & $\begin{array}{l}-0.0211^{* *} \\
(0.00974)\end{array}$ & $\begin{array}{c}-0.00614^{* *} \\
(0.00223)\end{array}$ & $\begin{array}{l}0.00918 \\
(0.0167)\end{array}$ & $\begin{array}{r}-0.000485 \\
(0.00946)\end{array}$ & $\begin{array}{c}-0.0544^{* *} \\
(0.0202)\end{array}$ & $\begin{array}{c}0.0765^{* * *} \\
(0.0247)\end{array}$ \\
\hline 0_in_3months & $\begin{array}{c}-0.0634^{* * *} \\
(0.00886)\end{array}$ & $\begin{array}{l}-0.0293^{* * *} \\
(0.00382)\end{array}$ & $\begin{array}{c}-0.00156 \\
(0.0111)\end{array}$ & $\begin{array}{l}-0.0177^{* * *} \\
(0.00547)\end{array}$ & $\begin{array}{c}-0.0631^{* * *} \\
(0.0125)\end{array}$ & $\begin{array}{c}-0.00566^{* * *} \\
(0.00132)\end{array}$ & $\begin{array}{r}-0.00674 \\
(0.0120)\end{array}$ & $\begin{array}{l}-0.0174^{* *} \\
(0.00601)\end{array}$ & $\begin{array}{c}0.00392 \\
(0.00558)\end{array}$ & $\begin{array}{l}-0.00338 \\
(0.00408)\end{array}$ & $\begin{array}{c}-0.00418^{* * *} \\
(0.00102)\end{array}$ & $\begin{array}{c}0.00917 \\
(0.00717)\end{array}$ & $\begin{array}{l}-0.00483 \\
(0.00411)\end{array}$ & $\begin{array}{c}-0.00386 \\
(0.00906)\end{array}$ & $\begin{array}{c}0.0162^{*} \\
(0.00912)\end{array}$ \\
\hline & & & & & & & & & & & & & & & \\
\hline 1_week & $\begin{array}{c}0.0161 \\
(0.0146)\end{array}$ & $\begin{array}{l}0.000467 \\
(0.00914)\end{array}$ & $\begin{array}{c}0.0158 \\
(0.0241)\end{array}$ & $\begin{array}{c}0.00140 \\
(0.00409)\end{array}$ & $\begin{array}{c}0.00343 \\
(0.00571)\end{array}$ & $\begin{array}{c}-0.00853^{\star *} \\
(0.00296)\end{array}$ & $\begin{array}{c}0.00453 \\
(0.00767)\end{array}$ & $\begin{array}{c}0.00228 \\
(0.00536)\end{array}$ & $\begin{array}{c}-0.00374 \\
(0.00288)\end{array}$ & $\begin{array}{l}0.00690^{* *} \\
(0.00258)\end{array}$ & $\begin{array}{c}-5.36 e-05 \\
(0.000631)\end{array}$ & $\begin{array}{l}0.00617 \\
(0.0104)\end{array}$ & $\begin{array}{c}0.00213 \\
(0.00358)\end{array}$ & $\begin{array}{c}0.0134 \\
(0.00920)\end{array}$ & $\begin{array}{l}-0.0218 \\
(0.0126)\end{array}$ \\
\hline 1or3_ & $\begin{array}{l}0.0291^{* * *} \\
(0.00843)\end{array}$ & $\begin{array}{c}0.0140 \\
(0.0158)\end{array}$ & $\begin{array}{l}0.0226^{*} \\
(0.0106)\end{array}$ & $\begin{array}{l}0.000137 \\
(0.00362)\end{array}$ & $\begin{array}{l}0.00813 \\
(0.0116)\end{array}$ & $\begin{array}{c}0.00573 \\
(0.00408)\end{array}$ & $\begin{array}{l}0.00453 \\
(0.0128)\end{array}$ & $\begin{array}{c}0.00111 \\
(0.00597)\end{array}$ & $\begin{array}{c}-0.00483 \\
(0.00582)\end{array}$ & $\begin{array}{c}0.00302 \\
(0.00485)\end{array}$ & $\begin{array}{c}0.000786 \\
(0.000700)\end{array}$ & $\begin{array}{c}-0.00470 \\
(0.00680)\end{array}$ & $\begin{array}{l}-0.00267 \\
(0.00282)\end{array}$ & $\begin{array}{c}0.00868 \\
(0.00879)\end{array}$ & $\begin{array}{l}-0.0418^{* \star *} \\
(0.00787)\end{array}$ \\
\hline never & $\begin{array}{c}0.155^{\star * \star} \\
(0.00967)\end{array}$ & $\begin{array}{l}0.0597^{* * *} \\
(0.00465)\end{array}$ & $\begin{array}{c}0.0643^{* \star *} \\
(0.0131)\end{array}$ & $\begin{array}{l}0.0291^{* *} \\
(0.00548)\end{array}$ & $\begin{array}{l}0.0280^{* * *} \\
(0.00605)\end{array}$ & $\begin{array}{l}0.0230^{* * *} \\
(0.00634)\end{array}$ & $\begin{array}{c}0.0782^{* * *} \\
(0.0103)\end{array}$ & $\begin{array}{c}0.0230^{* *} \\
(0.00777)\end{array}$ & $\begin{array}{l}0.0130^{* \star *} \\
(0.00257)\end{array}$ & $\begin{array}{c}0.0122 \\
(0.00706)\end{array}$ & $\begin{array}{c}0.00710^{* * *} \\
(0.00184)\end{array}$ & $\begin{array}{c}0.00811 \\
(0.00607)\end{array}$ & $\begin{array}{l}0.00903^{\star *} \\
(0.00349)\end{array}$ & $\begin{array}{l}0.0532^{* * *} \\
(0.00765)\end{array}$ & $\begin{array}{c}-0.104^{* * *} \\
(0.0102)\end{array}$ \\
\hline Smoking & $\begin{array}{c}-0.0320^{* \star} \\
(0.0128)\end{array}$ & $\begin{array}{c}-0.0233^{\star \star \star} \\
(0.00478)\end{array}$ & $\begin{array}{c}-0.0456^{* * *} \\
(0.0111)\end{array}$ & $\begin{array}{c}0.00529 \\
(0.00561)\end{array}$ & $\begin{array}{l}-0.00852 \\
(0.00518)\end{array}$ & $\begin{array}{c}-0.00900^{* *} \\
(0.00316)\end{array}$ & $\begin{array}{l}-0.0211^{* *} \\
(0.00891)\end{array}$ & $\begin{array}{l}-0.00778 \\
(0.00549)\end{array}$ & $\begin{array}{l}-0.00753^{*} \\
(0.00377)\end{array}$ & $\begin{array}{l}0.0149^{\star \star \star} \\
(0.00358)\end{array}$ & $\begin{array}{l}-0.00208^{\star *} \\
(0.000707)\end{array}$ & $\begin{array}{l}-7.17 \mathrm{e}-05 \\
(0.00786)\end{array}$ & $\begin{array}{l}0.000322 \\
(0.00136)\end{array}$ & $\begin{array}{l}-0.00144 \\
(0.00715)\end{array}$ & $\begin{array}{l}0.0244^{*} \\
(0.0119)\end{array}$ \\
\hline & & & & & & & & & & & & & & & \\
\hline Ohoce & $\begin{array}{l}0.0674^{* * *} \\
(0.00707)\end{array}$ & $\begin{array}{c}0.0186^{\star *} \\
(0.00671)\end{array}$ & $\begin{array}{c}0.158^{\star * *} \\
(0.00989)\end{array}$ & $\begin{array}{c}-0.00325 \\
(0.00354)\end{array}$ & $\begin{array}{l}0.0613^{\star \star *} \\
(0.00517)\end{array}$ & $\begin{array}{l}0.0164^{\star * \star} \\
(0.00455)\end{array}$ & $\begin{array}{l}0.0293^{\star * \star} \\
(0.00689)\end{array}$ & $\begin{array}{c}-0.0145^{\star * *} \\
(0.00308)\end{array}$ & $\begin{array}{c}-0.00322 \\
(0.00245)\end{array}$ & $\begin{array}{l}0.00267^{\star *} \\
(0.00119)\end{array}$ & $\begin{array}{l}-0.000982 \\
(0.00116)\end{array}$ & $\begin{array}{c}0.00592^{\star * *} \\
(0.00189)\end{array}$ & $\begin{array}{l}0.000186 \\
(0.00255)\end{array}$ & $\begin{array}{c}0.00971^{* * *} \\
(0.00319)\end{array}$ & $\begin{array}{c}-0.0810^{\star \star \star} \\
(0.00687)\end{array}$ \\
\hline Female & $\begin{array}{c}0.0116 \\
(0.00778)\end{array}$ & $\begin{array}{c}-0.0806^{* \star *} \\
(0.0118)\end{array}$ & $\begin{array}{l}0.0279^{* \star *} \\
(0.00581)\end{array}$ & $\begin{array}{c}-0.0193^{* * *} \\
(0.00609)\end{array}$ & $\begin{array}{c}-0.0310^{* * *} \\
(0.00279)\end{array}$ & $\begin{array}{c}0.00782 \\
(0.00480)\end{array}$ & $\begin{array}{c}0.0901^{* \star *} \\
(0.0168)\end{array}$ & $\begin{array}{c}0.0760^{* * *} \\
(0.0163)\end{array}$ & $\begin{array}{l}0.0168^{* \star *} \\
(0.00540)\end{array}$ & $\begin{array}{c}-0.0178^{* * *} \\
(0.00237)\end{array}$ & $\begin{array}{l}-0.00259 \\
(0.00150)\end{array}$ & $\begin{array}{l}0.0315^{\star \star *} \\
(0.00970)\end{array}$ & $\begin{array}{c}0.00306 \\
(0.00189)\end{array}$ & $\begin{array}{l}0.0366^{*} \\
(0.0193)\end{array}$ & $\begin{array}{l}-0.0176 \\
(0.0111)\end{array}$ \\
\hline Age55_59 & $\begin{array}{l}0.0320^{* * *} \\
(0.00708)\end{array}$ & $\begin{array}{c}0.00396 \\
(0.00513)\end{array}$ & $\begin{array}{l}0.0695^{\star \star \star} \\
(0.00769)\end{array}$ & $\begin{array}{l}0.00599^{* *} \\
(0.00233)\end{array}$ & $\begin{array}{c}0.00751 \\
(0.00493)\end{array}$ & $\begin{array}{l}-0.00258 \\
(0.00661)\end{array}$ & $\begin{array}{l}0.0454^{* \star *} \\
(0.00460)\end{array}$ & $\begin{array}{l}0.0224^{* * *} \\
(0.00597)\end{array}$ & $\begin{array}{c}0.0108^{*} \\
(0.00608)\end{array}$ & $\begin{array}{c}0.00455 \\
(0.00303)\end{array}$ & $\begin{array}{l}-0.000436 \\
(0.000768)\end{array}$ & $\begin{array}{c}0.00369 \\
(0.00392)\end{array}$ & $\begin{array}{c}0.00354 \\
(0.00372)\end{array}$ & $\begin{array}{c}0.00421 \\
(0.00901)\end{array}$ & $\begin{array}{l}-0.103^{* \star *} \\
(0.00827)\end{array}$ \\
\hline Age60_64 & $\begin{array}{l}0.0242^{\star *} \\
(0.00918)\end{array}$ & $\begin{array}{c}0.0109 \\
(0.00883)\end{array}$ & $\begin{array}{l}0.101^{* \star \star} \\
(0.0152)\end{array}$ & $\begin{array}{c}0.00261 \\
(0.00296)\end{array}$ & $\begin{array}{l}0.0162^{* \star *} \\
(0.00406)\end{array}$ & $\begin{array}{l}-0.00481 \\
(0.00782)\end{array}$ & $\begin{array}{c}0.0687^{\star \star \star} \\
(0.0145)\end{array}$ & $\begin{array}{c}0.0360^{\star \star \star} \\
(0.0105)\end{array}$ & $\begin{array}{l}0.0119^{* \star \star} \\
(0.00313)\end{array}$ & $\begin{array}{c}0.00678 \\
(0.00394)\end{array}$ & $\begin{array}{c}0.00129 \\
(0.00142)\end{array}$ & $\begin{array}{l}0.0211^{* \star \star} \\
(0.00621)\end{array}$ & $\begin{array}{l}-5.48 \mathrm{e}-05 \\
(0.00187)\end{array}$ & $\begin{array}{c}-0.0387^{\star \star *} \\
(0.00709)\end{array}$ & $\begin{array}{c}-0.132^{\star \star *} \\
(0.0132)\end{array}$ \\
\hline Age65_69 & $\begin{array}{c}0.0164 \\
(0.0266)\end{array}$ & $\begin{array}{l}0.0257^{\star \star \star} \\
(0.00437)\end{array}$ & $\begin{array}{l}0.157^{* * *} \\
(0.0195)\end{array}$ & $\begin{array}{c}0.00603 \\
(0.00492)\end{array}$ & $\begin{array}{l}0.0293^{\star * *} \\
(0.00866)\end{array}$ & $\begin{array}{l}-0.0139^{* \star} \\
(0.00545)\end{array}$ & $\begin{array}{l}0.0619^{* \star} \\
(0.0237)\end{array}$ & $\begin{array}{c}0.0493^{* \star *} \\
(0.0109)\end{array}$ & $\begin{array}{c}0.0167^{* *} \\
(0.00716)\end{array}$ & $\begin{array}{r}-0.000796 \\
(0.00533)\end{array}$ & $\begin{array}{l}0.000600 \\
(0.00239)\end{array}$ & $\begin{array}{l}0.0550^{\star \star \star} \\
(0.00785)\end{array}$ & $\begin{array}{r}-0.000434 \\
(0.00325)\end{array}$ & $\begin{array}{c}-0.0372^{\star \star \star} \\
(0.00723)\end{array}$ & $\begin{array}{c}-0.146^{\star \star \star} \\
(0.0173)\end{array}$ \\
\hline$A c$ & $\begin{array}{l}0.0558^{\star} \\
(0.0260)\end{array}$ & $\begin{array}{l}0.0668^{* \star *} \\
(0.00410)\end{array}$ & $\begin{array}{l}0.188^{\star \star \star} \\
(0.0172)\end{array}$ & $\begin{array}{c}0.0148^{*} \\
(0.00784)\end{array}$ & $\begin{array}{l}0.0275^{\star \star \star} \\
(0.00845)\end{array}$ & $\begin{array}{l}-0.0153^{* *} \\
(0.00700)\end{array}$ & $\begin{array}{c}0.0687^{* \star *} \\
(0.0176)\end{array}$ & $\begin{array}{c}0.0630^{* \star *} \\
(0.0160)\end{array}$ & $\begin{array}{l}0.0248^{\star \star *} \\
(0.00767)\end{array}$ & $\begin{array}{c}0.00136 \\
(0.00484)\end{array}$ & $\begin{array}{c}0.00168 \\
(0.00299)\end{array}$ & $\begin{array}{c}0.0846^{* \star *} \\
(0.0130)\end{array}$ & $\begin{array}{l}0.0116^{\star \star \star} \\
(0.00375)\end{array}$ & $\begin{array}{l}-0.0349^{*} \\
(0.0164)\end{array}$ & $\begin{array}{c}-0.204^{\star \star \star} \\
(0.0150)\end{array}$ \\
\hline Age75_79 & $\begin{array}{c}0.0716 \\
(0.0406)\end{array}$ & $\begin{array}{c}0.0883^{* *} \\
(0.0107)\end{array}$ & $\begin{array}{l}0.176^{\star \star \star} \\
(0.0172)\end{array}$ & $\begin{array}{l}0.0369^{* \star *} \\
(0.00527)\end{array}$ & $\begin{array}{c}0.0245 \\
(0.0147)\end{array}$ & $\begin{array}{c}-0.0135 \\
(0.00906)\end{array}$ & $\begin{array}{c}0.0900^{\star \star \star} \\
(0.0255)\end{array}$ & $\begin{array}{c}0.0690^{* \star *} \\
(0.0112)\end{array}$ & $\begin{array}{c}0.0493^{* \star *} \\
(0.0143)\end{array}$ & $\begin{array}{c}0.0155^{*} \\
(0.00822)\end{array}$ & $\begin{array}{l}-0.00138 \\
(0.00257)\end{array}$ & $\begin{array}{l}0.167^{* \star \star} \\
(0.0221)\end{array}$ & $\begin{array}{l}0.0148^{* \star *} \\
(0.00352)\end{array}$ & $\begin{array}{l}-0.0181 \\
(0.0188)\end{array}$ & $\begin{array}{c}-0.219^{* \star *} \\
(0.0166)\end{array}$ \\
\hline AgeAbove_80 & $\begin{array}{l}0.0656^{*} \\
(0.0316)\end{array}$ & $\begin{array}{l}0.108^{\star \star \star} \\
(0.0130)\end{array}$ & $\begin{array}{l}0.142^{* \star \star} \\
(0.0158)\end{array}$ & $\begin{array}{l}0.0386^{\star \star \star} \\
(0.00369)\end{array}$ & $\begin{array}{l}0.0274^{*} \\
(0.0111)\end{array}$ & $\begin{array}{c}-0.0180 \\
(0.0115)\end{array}$ & $\begin{array}{c}0.0833^{\star \star \star} \\
(0.0245)\end{array}$ & $\begin{array}{c}0.0789^{\star * \star} \\
(0.0119)\end{array}$ & $\begin{array}{c}0.0474^{\star \star \star} \\
(0.0115)\end{array}$ & $\begin{array}{c}0.00735 \\
(0.00969)\end{array}$ & $\begin{array}{c}0.00705 \\
(0.00620)\end{array}$ & $\begin{array}{l}0.251^{\star \star \star} \\
(0.0320)\end{array}$ & $\begin{array}{l}0.0388^{\star \star \star} \\
(0.00224)\end{array}$ & $\begin{array}{c}-0.0381^{* \star *} \\
(0.00916)\end{array}$ & $\begin{array}{c}-0.228^{* \star \star} \\
(0.0123)\end{array}$ \\
\hline Married & $\begin{array}{l}-0.0133 \\
(0.0139)\end{array}$ & $\begin{array}{c}0.0118^{*} \\
(0.00655)\end{array}$ & $\begin{array}{l}0.00751 \\
(0.0214)\end{array}$ & $\begin{array}{c}0.00608 \\
(0.00828)\end{array}$ & $\begin{array}{c}-0.00986 \\
(0.00669)\end{array}$ & $\begin{array}{c}0.00865 \\
(0.00913)\end{array}$ & $\begin{array}{c}0.0188 \\
(0.0129)\end{array}$ & $\begin{array}{l}-0.0122^{*} \\
(0.00672)\end{array}$ & $\begin{array}{l}0.00400 \\
(0.0106)\end{array}$ & $\begin{array}{l}0.000375 \\
(0.0106)\end{array}$ & $\begin{array}{c}0.00272 \\
(0.00270)\end{array}$ & $\begin{array}{l}-0.00854 \\
(0.00773)\end{array}$ & $\begin{array}{c}0.00296 \\
(0.00582)\end{array}$ & $\begin{array}{c}-0.0527^{\star * *} \\
(0.0135)\end{array}$ & $\begin{array}{l}-0.00591 \\
(0.0206)\end{array}$ \\
\hline Reg_Partnership & $\begin{array}{l}-0.0127 \\
(0.0233)\end{array}$ & $\begin{array}{c}-0.0355^{\star *} \\
(0.0143)\end{array}$ & $\begin{array}{c}-0.0668^{\star * \star} \\
(0.0186)\end{array}$ & $\begin{array}{l}-0.0128 \\
(0.0110)\end{array}$ & $\begin{array}{l}-0.0219^{\star \star} \\
(0.00844)\end{array}$ & $\begin{array}{c}0.00492 \\
(0.00954)\end{array}$ & $\begin{array}{c}0.0171 \\
(0.0174)\end{array}$ & $\begin{array}{c}-0.0146 \\
(0.00971)\end{array}$ & $\begin{array}{c}0.0178 \\
(0.0143)\end{array}$ & $\begin{array}{l}0.00451 \\
(0.0108)\end{array}$ & $\begin{array}{c}0.00918^{\star \star \star} \\
(0.00260)\end{array}$ & $\begin{array}{l}-0.0126 \\
(0.0238)\end{array}$ & $\begin{array}{l}-0.00969^{*} \\
(0.00504)\end{array}$ & $\begin{array}{c}-0.0673^{\star \star \star} \\
(0.0142)\end{array}$ & $\begin{array}{c}0.0257 \\
(0.0177)\end{array}$ \\
\hline
\end{tabular}




\begin{tabular}{|c|c|c|c|c|c|c|c|c|c|c|c|c|c|c|c|}
\hline Separated & $\begin{array}{c}0.0275 \\
(0.0372)\end{array}$ & $\begin{array}{l}0.00376 \\
(0.0223)\end{array}$ & $\begin{array}{l}-0.0162 \\
(0.0329)\end{array}$ & $\begin{array}{c}0.0307 \\
(0.0251)\end{array}$ & $\begin{array}{c}0.0540 \\
(0.0323)\end{array}$ & $\begin{array}{l}0.00321 \\
(0.0157)\end{array}$ & $\begin{array}{l}0.0863^{* *} \\
(0.0392)\end{array}$ & $\begin{array}{l}-0.0195 \\
(0.0184)\end{array}$ & $\begin{array}{l}0.00667 \\
(0.0274)\end{array}$ & $\begin{array}{l}0.0713^{*} \\
(0.0373)\end{array}$ & $\begin{array}{l}0.00881 \\
(0.0104)\end{array}$ & $\begin{array}{c}0.0213 \\
(0.0273)\end{array}$ & $\begin{array}{c}0.00262 \\
(0.00900)\end{array}$ & $\begin{array}{c}-0.0578 \\
(0.0346)\end{array}$ & $\begin{array}{c}-0.0699^{\star * *} \\
(0.0210)\end{array}$ \\
\hline Divorced & $\begin{array}{c}0.0593^{\star \star *} \\
(0.0191)\end{array}$ & $\begin{array}{l}0.0479^{* *} \\
(0.0218)\end{array}$ & $\begin{array}{c}0.0123 \\
(0.0231)\end{array}$ & $\begin{array}{c}0.0118 \\
(0.0123)\end{array}$ & $\begin{array}{c}0.0153 \\
(0.0109)\end{array}$ & $\begin{array}{l}0.0233^{* *} \\
(0.0106)\end{array}$ & $\begin{array}{c}0.0654^{* * \star} \\
(0.0202)\end{array}$ & $\begin{array}{l}0.00500 \\
(0.0133)\end{array}$ & $\begin{array}{c}0.0203 \\
(0.0191)\end{array}$ & $\begin{array}{c}0.00703 \\
(0.00757)\end{array}$ & $\begin{array}{c}0.00229 \\
(0.00218)\end{array}$ & $\begin{array}{c}-0.000526 \\
(0.0106)\end{array}$ & $\begin{array}{c}0.0106 \\
(0.0109)\end{array}$ & $\begin{array}{l}-0.0240 \\
(0.0161)\end{array}$ & $\begin{array}{c}-0.0505^{* *} \\
(0.0225)\end{array}$ \\
\hline Widowed & $\begin{array}{r}-0.00798 \\
(0.0158)\end{array}$ & $\begin{array}{l}0.00676 \\
(0.0104)\end{array}$ & $\begin{array}{c}0.0453 \\
(0.0352)\end{array}$ & $\begin{array}{c}0.00636 \\
(0.00880)\end{array}$ & $\begin{array}{c}0.0182^{* *} \\
(0.00813)\end{array}$ & $\begin{array}{l}0.00820 \\
(0.0152)\end{array}$ & $\begin{array}{c}0.0246 \\
(0.0141)\end{array}$ & $\begin{array}{l}-0.0120 \\
(0.0121)\end{array}$ & $\begin{array}{l}-0.00126 \\
(0.0106)\end{array}$ & $\begin{array}{c}0.00588 \\
(0.00847)\end{array}$ & $\begin{array}{l}0.00564^{* *} \\
(0.00245)\end{array}$ & $\begin{array}{c}0.0152 \\
(0.0160)\end{array}$ & $\begin{array}{c}0.0139^{*} \\
(0.00663)\end{array}$ & $\begin{array}{c}-0.0572^{\star \star *} \\
(0.0181)\end{array}$ & $\begin{array}{c}-0.0239 \\
(0.0269)\end{array}$ \\
\hline Retired & $\begin{array}{c}-0.169^{* \star \star} \\
(0.0203)\end{array}$ & $\begin{array}{c}-0.0192^{*} \\
(0.00988)\end{array}$ & $\begin{array}{c}-0.00766 \\
(0.0164)\end{array}$ & $\begin{array}{l}-0.0280 \\
(0.0162)\end{array}$ & $\begin{array}{l}-0.0218^{*} \\
(0.0109)\end{array}$ & $\begin{array}{l}-0.00871 \\
(0.00895)\end{array}$ & $\begin{array}{l}-0.0562^{*} \\
(0.0310)\end{array}$ & $\begin{array}{c}-0.0263^{* * *} \\
(0.00699)\end{array}$ & $\begin{array}{l}-0.0193^{\star *} \\
(0.00772)\end{array}$ & $\begin{array}{l}-0.00508 \\
(0.00731)\end{array}$ & $\begin{array}{r}-0.000676 \\
(0.00431)\end{array}$ & $\begin{array}{r}0.000221 \\
(0.0108)\end{array}$ & $\begin{array}{c}-0.00738 \\
(0.00490)\end{array}$ & $\begin{array}{c}-0.0672^{* * *} \\
(0.0140)\end{array}$ & $\begin{array}{c}0.0863^{* * *} \\
(0.0145)\end{array}$ \\
\hline Employed & $\begin{array}{c}-0.284^{* \star \star} \\
(0.0193)\end{array}$ & $\begin{array}{c}-0.0628^{* \star *} \\
(0.00649)\end{array}$ & $\begin{array}{l}-0.0304^{*} \\
(0.0131)\end{array}$ & $\begin{array}{c}-0.0499^{\star * *} \\
(0.0113)\end{array}$ & $\begin{array}{c}-0.0416^{* \star *} \\
(0.0102)\end{array}$ & $\begin{array}{l}-0.0147 \\
(0.0122)\end{array}$ & $\begin{array}{c}-0.0926^{* \star \star} \\
(0.0168)\end{array}$ & $\begin{array}{c}-0.0295^{* \star *} \\
(0.00588)\end{array}$ & $\begin{array}{l}-0.0434^{* \star *} \\
(0.00575)\end{array}$ & $\begin{array}{c}-0.0180^{*} \\
(0.00983)\end{array}$ & $\begin{array}{l}-0.00436 \\
(0.00318)\end{array}$ & $\begin{array}{c}-0.0114 \\
(0.0119)\end{array}$ & $\begin{array}{c}-0.0127^{* * \star} \\
(0.00247)\end{array}$ & $\begin{array}{l}-0.111^{* * \star} \\
(0.0100)\end{array}$ & $\begin{array}{l}0.179^{* \star \star} \\
(0.0156)\end{array}$ \\
\hline Homemaker & $\begin{array}{c}-0.201^{* * *} \\
(0.0166)\end{array}$ & $\begin{array}{l}-0.0243 \\
(0.0159)\end{array}$ & $\begin{array}{l}0.00832 \\
(0.0123)\end{array}$ & $\begin{array}{l}-0.0430^{* *} \\
(0.0172)\end{array}$ & $\begin{array}{c}-0.0245^{*} \\
(0.0101)\end{array}$ & $\begin{array}{l}-0.0230^{* \star *} \\
(0.00659)\end{array}$ & $\begin{array}{c}-0.0654^{* *} \\
(0.0268)\end{array}$ & $\begin{array}{l}-0.0145 \\
(0.0157)\end{array}$ & $\begin{array}{c}-0.0293^{*} \\
(0.0100)\end{array}$ & $\begin{array}{l}-0.0164^{*} \\
(0.00760)\end{array}$ & $\begin{array}{l}-0.00529 \\
(0.00370)\end{array}$ & $\begin{array}{l}-0.0138 \\
(0.0160)\end{array}$ & $\begin{array}{l}-0.00718^{*} \\
(0.00384)\end{array}$ & $\begin{array}{c}-0.0972^{\star \star *} \\
(0.0185)\end{array}$ & $\begin{array}{l}0.103^{* \star \star} \\
(0.0143)\end{array}$ \\
\hline Ot & $\begin{array}{l}-0.151^{* * \star} \\
(0.0288)\end{array}$ & $\begin{array}{l}-0.0407 \\
(0.0260)\end{array}$ & $\begin{array}{l}-0.0501 \\
(0.0361)\end{array}$ & $\begin{array}{c}-0.0116 \\
(0.00773)\end{array}$ & $\begin{array}{l}-0.0188 \\
(0.0187)\end{array}$ & $\begin{array}{c}-0.0340^{* \star} \\
(0.0154)\end{array}$ & $\begin{array}{c}-0.0527 \\
(0.0308)\end{array}$ & $\begin{array}{c}0.0137 \\
(0.0151)\end{array}$ & $\begin{array}{l}-0.00523 \\
(0.0205)\end{array}$ & $\begin{array}{c}-0.00637 \\
(0.00959)\end{array}$ & $\begin{array}{l}528 \\
508)\end{array}$ & $\begin{array}{l}-0.0136 \\
(0.0199)\end{array}$ & $\begin{array}{c}-0.00739 \\
(0.00796)\end{array}$ & $\begin{array}{l}-0.0660 \\
(0.0388)\end{array}$ & $\begin{array}{c}0.0291 \\
(0.0316)\end{array}$ \\
\hline N_Children & $\begin{array}{c}-0.00877^{* *} \\
(0.00368)\end{array}$ & $\begin{array}{c}0.00131 \\
(0.00211)\end{array}$ & $\begin{array}{l}0.000963 \\
(0.00159)\end{array}$ & $\begin{array}{l}0.00293^{*} \\
(0.00136)\end{array}$ & $\begin{array}{c}0.00222 \\
(0.00341)\end{array}$ & $\begin{array}{l}0.000724 \\
(0.00184)\end{array}$ & $\begin{array}{c}0.00320 \\
(0.00220)\end{array}$ & $\begin{array}{l}-0.00214^{* * *} \\
(0.000693)\end{array}$ & $\begin{array}{l}-0.00227 \\
(0.00181)\end{array}$ & $\begin{array}{c}-0.00151 \\
(0.000894)\end{array}$ & $\begin{array}{l}-5.00 \mathrm{e}-05 \\
(0.000667)\end{array}$ & $\begin{array}{l}-0.00324 \\
(0.00226)\end{array}$ & $\begin{array}{l}-0.00221 \\
(0.00192)\end{array}$ & $\begin{array}{l}-0.00415^{\star} \\
(0.00224)\end{array}$ & $\begin{array}{l}0.00881^{* *} \\
(0.00360)\end{array}$ \\
\hline N_Grandchildren & $\begin{array}{c}0.00564^{* * *} \\
(0.00164)\end{array}$ & $\begin{array}{l}0.00319^{\star *} \\
(0.00140)\end{array}$ & $\begin{array}{l}0.000415 \\
(0.00148)\end{array}$ & $\begin{array}{c}-0.000876^{*} \\
(0.000410)\end{array}$ & $\begin{array}{l}0.00206^{* *} \\
(0.000630)\end{array}$ & $\begin{array}{c}0.00129^{*} \\
(0.000609)\end{array}$ & $\begin{array}{l}0.00203^{*} \\
(0.00109)\end{array}$ & $\begin{array}{l}0.000742 \\
(0.00118)\end{array}$ & $\begin{array}{c}0.000467 \\
(0.000825)\end{array}$ & $\begin{array}{c}0.00116 \\
(0.000784)\end{array}$ & $\begin{array}{c}-0.000122 \\
(0.000548)\end{array}$ & $\begin{array}{l}0.000175 \\
(0.00149)\end{array}$ & $\begin{array}{c}0.000359 \\
(0.000637)\end{array}$ & $\begin{array}{c}0.000161 \\
(0.000920)\end{array}$ & $\begin{array}{c}-0.00485^{\star \star *} \\
(0.00149)\end{array}$ \\
\hline Logincome & $\begin{array}{l}0.0198^{\star *} \\
(0.00809)\end{array}$ & $\begin{array}{c}0.00657^{* * *} \\
(0.00217)\end{array}$ & $\begin{array}{c}0.0119^{*} \\
(0.00624)\end{array}$ & $\begin{array}{l}0.00259^{* \star} \\
(0.000482)\end{array}$ & $\begin{array}{l}0.00527^{*} \\
(0.00190)\end{array}$ & $\begin{array}{l}0.00105^{*} \\
(0.000408)\end{array}$ & $\begin{array}{c}0.0103^{\star *} \\
(0.00429)\end{array}$ & $\begin{array}{c}0.00118 \\
(0.00101)\end{array}$ & $\begin{array}{l}0.00277^{* * *} \\
(0.000731)\end{array}$ & $\begin{array}{c}0.00126 \\
(0.000752)\end{array}$ & $\begin{array}{l}0.000623^{* *} \\
(0.000213)\end{array}$ & $\begin{array}{c}0.00752^{* * *} \\
(0.00222)\end{array}$ & $\begin{array}{l}0.00187^{* * *} \\
(0.000461)\end{array}$ & $\begin{array}{l}0.00641^{* * *} \\
(0.00151)\end{array}$ & $\begin{array}{c}-0.0228^{* *} \\
(0.00829)\end{array}$ \\
\hline
\end{tabular}

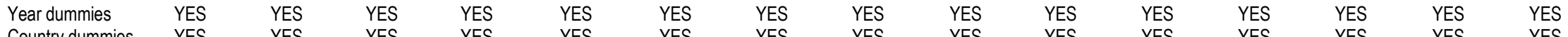

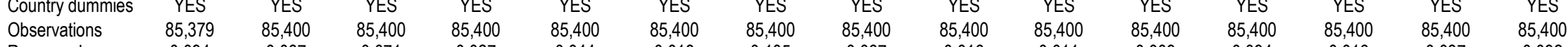

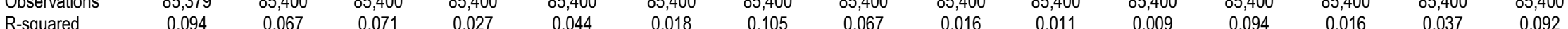

Omitted benchmarks: unemployed for job status, never_married for marital status, Austria for country dummies, age50_54 for age dummies, almost_every_day for drinking habits, <1_week for sport activities. Robust standard errors in parentheses clustered at country level. ${ }^{* *} p<0.01,{ }^{* *} p<0.05,{ }^{*} p<0.1$ 
Table A5a - Education years and health functionalities (IV estimates)

\begin{tabular}{|c|c|c|c|c|c|c|c|c|c|c|c|c|c|c|c|c|}
\hline \multirow[b]{3}{*}{ VARIABLES } & \multirow{3}{*}{\multicolumn{2}{|c|}{ (1) casp }} & \multirow{2}{*}{\multicolumn{2}{|c|}{$\begin{array}{l}\text { (3) } \\
\text { healthinsat }\end{array}$}} & \multirow{2}{*}{\multicolumn{2}{|c|}{$\begin{array}{cr}(5) & (6) \\
\text { n_chronicdeseases }\end{array}$}} & (7) & (8) & (9) & (10) & (11) & (12) & (13) & (14) & (15) & (16) \\
\hline & & & & & & & \multicolumn{2}{|c|}{ adla } & \multicolumn{2}{|c|}{ iadla } & \multicolumn{2}{|c|}{ n_doctorvisits } & \multicolumn{2}{|c|}{ n_wordsrecalled } & \multicolumn{2}{|c|}{ mobilityind } \\
\hline & & & healthinsat & eduyears & n_chronicdes. & eduyears & adla & eduyears & iadla & eduyears & n_doctorvisits & eduyears & n_wordsrec. & eduyears & mobilityind & eduyears \\
\hline Eduyears & $\begin{array}{c}0.726^{\star * *} \\
(0.143)\end{array}$ & & $\begin{array}{l}-0.147^{* * *} \\
(0.0262)\end{array}$ & & $\begin{array}{l}-0.171^{* * *} \\
(0.0317)\end{array}$ & & $\begin{array}{l}-0.277^{\star * *} \\
(0.0251)\end{array}$ & & $\begin{array}{l}-0.193^{* * *} \\
(0.0153)\end{array}$ & & $\begin{array}{c}-0.00137 \\
(0.148)\end{array}$ & & $\begin{array}{l}0.524^{\star \star \star} \\
(0.0618)\end{array}$ & & $\begin{array}{l}-0.297^{* * *} \\
(0.0319)\end{array}$ & \\
\hline First_Qtr & & $\begin{array}{c}-0.0995^{\star *} \\
(0.0403)\end{array}$ & & $\begin{array}{l}-0.0763^{*} \\
(0.0460)\end{array}$ & & $\begin{array}{l}-0.0762^{*} \\
(0.0432)\end{array}$ & & $\begin{array}{l}-0.0310 \\
(0.0191)\end{array}$ & & $\begin{array}{c}-0.0382^{* * *} \\
(0.0136)\end{array}$ & & $\begin{array}{l}-0.108^{* * *} \\
(0.0416)\end{array}$ & & $\begin{array}{c}-0.0916^{\star * *} \\
(0.0307)\end{array}$ & & $\begin{array}{l}-0.0362^{*} \\
(0.0197)\end{array}$ \\
\hline Age & & $\begin{array}{l}-0.105^{*} \\
(0.0605)\end{array}$ & & $\begin{array}{l}-0.141^{* *} \\
(0.0602)\end{array}$ & & $\begin{array}{l}-0.148^{* *} \\
(0.0635)\end{array}$ & & $\begin{array}{l}0.0487 \\
(0.0298)\end{array}$ & & $\begin{array}{l}0.116^{* * *} \\
(0.0230)\end{array}$ & & $\begin{array}{l}-0.112^{*} \\
(0.0628)\end{array}$ & & $\begin{array}{l}-0.0296 \\
(0.0517)\end{array}$ & & $\begin{array}{l}-0.0142 \\
(0.0439)\end{array}$ \\
\hline $\mathrm{Age}^{2}$ & & $\begin{array}{c}1.54 \mathrm{e}-05 \\
(0.000394)\end{array}$ & & $\begin{array}{c}0.000285 \\
(0.000394)\end{array}$ & & $\begin{array}{c}0.000334 \\
(0.000420)\end{array}$ & & $\begin{array}{l}-0.00112^{* * *} \\
(0.000185)\end{array}$ & & $\begin{array}{l}-0.00160^{* * *} \\
(0.000170)\end{array}$ & & $\begin{array}{c}6.70 \mathrm{e}-05 \\
(0.000414)\end{array}$ & & $\begin{array}{l}-0.000545^{*} \\
(0.000323)\end{array}$ & & $\begin{array}{c}-0.000660^{* *} \\
(0.000265)\end{array}$ \\
\hline Female & $\begin{array}{l}0.364^{* *} \\
(0.170)\end{array}$ & $\begin{array}{c}-0.783^{* * *} \\
(0.119)\end{array}$ & $\begin{array}{l}-0.105^{\star * *} \\
(0.0323)\end{array}$ & $\begin{array}{c}-0.789^{* * *} \\
(0.119)\end{array}$ & $\begin{array}{c}-0.0780^{* *} \\
(0.0372)\end{array}$ & $\begin{array}{c}-0.790^{\star * *} \\
(0.119)\end{array}$ & $\begin{array}{l}-0.212^{* * *} \\
(0.0351)\end{array}$ & $\begin{array}{c}-0.754^{* * *} \\
(0.116)\end{array}$ & $\begin{array}{l}-0.156^{* * *} \\
(0.0254)\end{array}$ & $\begin{array}{c}-0.741^{* * *} \\
(0.114)\end{array}$ & $\begin{array}{l}0.216^{* *} \\
(0.0976)\end{array}$ & $\begin{array}{c}-0.784^{* * *} \\
(0.119)\end{array}$ & $\begin{array}{l}0.744^{\star \star *} \\
(0.0797)\end{array}$ & $\begin{array}{c}-0.769^{* * *} \\
(0.118)\end{array}$ & $\begin{array}{l}-0.125^{\star * *} \\
(0.0422)\end{array}$ & $\begin{array}{c}-0.766^{* * *} \\
(0.118)\end{array}$ \\
\hline DRINKING & & & & & & & & & & & & & & & & \\
\hline 5or6days_week & $\begin{array}{l}0.780^{* * *} \\
(0.139)\end{array}$ & & $\begin{array}{l}-0.141^{* * *} \\
(0.0181)\end{array}$ & & $\begin{array}{c}-0.0713^{* * *} \\
(0.0248)\end{array}$ & & $\begin{array}{l}-0.123^{* * *} \\
(0.0135)\end{array}$ & & $\begin{array}{c}-0.0615^{\star \star *} \\
(0.00821)\end{array}$ & & $\begin{array}{c}-1.198^{* * *} \\
(0.180)\end{array}$ & & $\begin{array}{l}0.203^{\star * *} \\
(0.0225)\end{array}$ & & $\begin{array}{l}-0.166^{\star * *} \\
(0.0186)\end{array}$ & \\
\hline 3or4days_week & $\begin{array}{l}0.937^{* * *} \\
(0.166)\end{array}$ & & $\begin{array}{l}-0.191^{* * *} \\
(0.0151)\end{array}$ & & $\begin{array}{c}-0.0865^{\star * *} \\
(0.0282)\end{array}$ & & $\begin{array}{l}-0.114^{* * *} \\
(0.0141)\end{array}$ & & $\begin{array}{l}-0.0602^{* \star *} \\
(0.00561)\end{array}$ & & $\begin{array}{c}-1.312^{* * *} \\
(0.185)\end{array}$ & & $\begin{array}{l}0.271^{* \star *} \\
(0.0348)\end{array}$ & & $\begin{array}{l}-0.195^{\star * *} \\
(0.0151)\end{array}$ & \\
\hline 1or2_week & $\begin{array}{l}1.151^{* * *} \\
(0.145)\end{array}$ & & $\begin{array}{l}-0.250^{* * *} \\
(0.0179)\end{array}$ & & $\begin{array}{l}-0.123^{* * *} \\
(0.0279)\end{array}$ & & $\begin{array}{l}-0.125^{\star * *} \\
(0.0115)\end{array}$ & & $\begin{array}{l}-0.0582^{* * *} \\
(0.00605)\end{array}$ & & $\begin{array}{c}-1.626^{* * *} \\
(0.242)\end{array}$ & & $\begin{array}{l}0.258^{\star * \star} \\
(0.0397)\end{array}$ & & $\begin{array}{l}-0.201^{* * *} \\
(0.0140)\end{array}$ & \\
\hline 1or2_month & $\begin{array}{l}1.061^{* * *} \\
(0.156)\end{array}$ & & $\begin{array}{l}-0.282^{* * *} \\
(0.0262)\end{array}$ & & $\begin{array}{l}-0.123^{\star * *} \\
(0.0308)\end{array}$ & & $\begin{array}{l}-0.125^{* * *} \\
(0.0134)\end{array}$ & & $\begin{array}{c}-0.0547^{\star * \star} \\
(0.00698)\end{array}$ & & $\begin{array}{c}-1.553^{\star * *} \\
(0.294)\end{array}$ & & $\begin{array}{l}0.314^{* \star *} \\
(0.0533)\end{array}$ & & $\begin{array}{l}-0.195^{\star * *} \\
(0.0149)\end{array}$ & \\
\hline$<1$ _month & $\begin{array}{c}0.740^{\star * \star} \\
(0.119)\end{array}$ & & $\begin{array}{c}-0.299^{* * *} \\
(0.0264)\end{array}$ & & $\begin{array}{c}-0.142^{* \star \star} \\
(0.0362)\end{array}$ & & $\begin{array}{l}-0.127^{* * *} \\
(0.0138)\end{array}$ & & $\begin{array}{c}-0.0559^{\star * *} \\
(0.00666)\end{array}$ & & $\begin{array}{c}-1.653^{* * *} \\
(0.362)\end{array}$ & & $\begin{array}{l}0.381^{* \star \star} \\
(0.0735)\end{array}$ & & $\begin{array}{l}-0.182^{* * *} \\
(0.0211)\end{array}$ & \\
\hline 0_in_3months & $\begin{array}{l}1.041^{* * *} \\
(0.0989)\end{array}$ & & $\begin{array}{c}-0.233^{* * *} \\
(0.0187)\end{array}$ & & $\begin{array}{c}-0.0835^{\star \star *} \\
(0.0247)\end{array}$ & & $\begin{array}{c}-0.133^{\star * \star} \\
(0.0126)\end{array}$ & & $\begin{array}{c}-0.0652^{\star * \star} \\
(0.00786)\end{array}$ & & $\begin{array}{c}-1.890^{* \star *} \\
(0.203)\end{array}$ & & $\begin{array}{l}0.222^{* * \star} \\
(0.0524)\end{array}$ & & $\begin{array}{c}-0.206^{\star * \star} \\
(0.0138)\end{array}$ & \\
\hline SPORT & & & & & & & & & & & & & & & & \\
\hline 1_week & $\begin{array}{c}-0.599^{* * *} \\
(0.113)\end{array}$ & & $\begin{array}{c}0.107^{* * *} \\
(0.00964)\end{array}$ & & $\begin{array}{l}0.0521^{* *} \\
(0.0209)\end{array}$ & & $\begin{array}{c}-0.00272 \\
(0.00461)\end{array}$ & & $\begin{array}{l}-0.00271 \\
(0.00216)\end{array}$ & & $\begin{array}{c}0.345^{* * *} \\
(0.108)\end{array}$ & & $\begin{array}{r}-0.00337 \\
(0.0304)\end{array}$ & & $\begin{array}{c}0.0348^{* * *} \\
(0.0106)\end{array}$ & \\
\hline 1or3_month & $\begin{array}{c}-0.867^{* * *} \\
(0.110)\end{array}$ & & $\begin{array}{c}0.164^{* * *} \\
(0.01000)\end{array}$ & & $\begin{array}{c}0.0993^{* * *} \\
(0.0203)\end{array}$ & & $\begin{array}{c}-0.00858 \\
(0.00739)\end{array}$ & & $\begin{array}{l}-0.00375 \\
(0.00367)\end{array}$ & & $\begin{array}{c}0.635^{* * *} \\
(0.135)\end{array}$ & & $\begin{array}{l}-0.0235 \\
(0.0354)\end{array}$ & & $\begin{array}{c}0.0752^{\star * *} \\
(0.0179)\end{array}$ & \\
\hline Hardlyever_never & $\begin{array}{c}-2.075^{* * *} \\
(0.180)\end{array}$ & & $\begin{array}{l}0.502^{* * *} \\
(0.0186)\end{array}$ & & $\begin{array}{l}0.410^{\star * \star} \\
(0.0390)\end{array}$ & & $\begin{array}{l}0.205^{\star \star *} \\
(0.0244)\end{array}$ & & $\begin{array}{l}0.0661^{* * *} \\
(0.00931)\end{array}$ & & $\begin{array}{c}2.537^{* * *} \\
(0.229)\end{array}$ & & $\begin{array}{l}-0.225^{\star * \star} \\
(0.0342)\end{array}$ & & $\begin{array}{l}0.438^{* *} \\
(0.0321)\end{array}$ & \\
\hline Smoking & $\begin{array}{c}-0.540^{* * *} \\
(0.0848)\end{array}$ & & $\begin{array}{c}0.0950^{* * *} \\
(0.0173)\end{array}$ & & $\begin{array}{c}-0.0365^{\star *} \\
(0.0176)\end{array}$ & & $\begin{array}{l}-0.0100^{* *} \\
(0.00480)\end{array}$ & & $\begin{array}{c}-0.00901^{\star * *} \\
(0.00225)\end{array}$ & & $\begin{array}{c}-0.604^{* * *} \\
(0.133)\end{array}$ & & $\begin{array}{c}-0.0746^{\star * *} \\
(0.0209)\end{array}$ & & $\begin{array}{l}0.0400^{* * *} \\
(0.00878)\end{array}$ & \\
\hline Overweight_Obese & $\begin{array}{c}-0.267^{* * *} \\
(0.0684)\end{array}$ & & $\begin{array}{l}0.164^{* * *} \\
(0.0131)\end{array}$ & & $\begin{array}{l}0.361^{\star \star \star} \\
(0.0114)\end{array}$ & & $\begin{array}{l}0.0238^{\star * \star} \\
(0.00888)\end{array}$ & & $\begin{array}{c}-0.0165^{\star \star *} \\
(0.00475)\end{array}$ & & $\begin{array}{l}0.691^{* \star *} \\
(0.0955)\end{array}$ & & $\begin{array}{l}-0.0282 \\
(0.0174)\end{array}$ & & $\begin{array}{l}0.134^{* * *} \\
(0.0133)\end{array}$ & \\
\hline Age55_59 & $\begin{array}{c}0.404^{* * *} \\
(0.105)\end{array}$ & & $\begin{array}{c}0.0273 \\
(0.0263)\end{array}$ & & $\begin{array}{l}0.104^{* * *} \\
(0.0350)\end{array}$ & & $\begin{array}{c}-0.103^{* * *} \\
(0.0209)\end{array}$ & & $\begin{array}{c}-0.0583^{* * *} \\
(0.0124)\end{array}$ & & $\begin{array}{c}0.164 \\
(0.166)\end{array}$ & & $\begin{array}{l}0.108^{\star * \star} \\
(0.0353)\end{array}$ & & $\begin{array}{c}-0.0920^{* * *} \\
(0.0183)\end{array}$ & \\
\hline Age60_64 & $\begin{array}{c}0.944^{* \star *} \\
(0.154)\end{array}$ & & $\begin{array}{l}-0.0447 \\
(0.0439)\end{array}$ & & $\begin{array}{l}0.163^{\star \star \star} \\
(0.0490)\end{array}$ & & $\begin{array}{c}-0.195^{\star \star *} \\
(0.0318)\end{array}$ & & $\begin{array}{c}-0.118^{* * *} \\
(0.0209)\end{array}$ & & $\begin{array}{c}0.116 \\
(0.246)\end{array}$ & & $\begin{array}{l}0.199^{\star \star \star} \\
(0.0399)\end{array}$ & & $\begin{array}{c}-0.190^{\star \star *} \\
(0.0258)\end{array}$ & \\
\hline Age65_69 & $\begin{array}{c}0.978^{* \star *} \\
(0.235)\end{array}$ & & $\begin{array}{l}-0.0531 \\
(0.0571)\end{array}$ & & $\begin{array}{l}0.245^{\star * \star} \\
(0.0610)\end{array}$ & & $\begin{array}{c}-0.290^{\star * *} \\
(0.0436)\end{array}$ & & $\begin{array}{c}-0.185^{\star * *} \\
(0.0310)\end{array}$ & & $\begin{array}{c}0.582 \\
(0.377)\end{array}$ & & $\begin{array}{l}0.213^{* * *} \\
(0.0482)\end{array}$ & & $\begin{array}{c}-0.250^{\star * *} \\
(0.0360)\end{array}$ & \\
\hline Age70_74 & $\begin{array}{c}0.888^{* \star *} \\
(0.273)\end{array}$ & & $\begin{array}{l}-0.0106 \\
(0.0667)\end{array}$ & & $\begin{array}{l}0.337^{\star \star \star} \\
(0.0721)\end{array}$ & & $\begin{array}{c}-0.400^{* * *} \\
(0.0583)\end{array}$ & & $\begin{array}{c}-0.271^{* * *} \\
(0.0438)\end{array}$ & & $\begin{array}{l}0.968^{* *} \\
(0.382)\end{array}$ & & $\begin{array}{l}0.116^{* *} \\
(0.0567)\end{array}$ & & $\begin{array}{c}-0.300^{\star \star *} \\
(0.0446)\end{array}$ & \\
\hline Age75_79 & $\begin{array}{l}0.656^{\star *} \\
(0.335)\end{array}$ & & $\begin{array}{c}0.0254 \\
(0.0826)\end{array}$ & & $\begin{array}{l}0.439^{\star * *} \\
(0.0922)\end{array}$ & & $\begin{array}{l}-0.482^{* * *} \\
(0.0708)\end{array}$ & & $\begin{array}{l}-0.342^{* * *} \\
(0.0545)\end{array}$ & & $\begin{array}{l}1.525^{\star \star \star} \\
(0.496)\end{array}$ & & $\begin{array}{c}0.0303 \\
(0.0654)\end{array}$ & & $\begin{array}{l}-0.295^{\star * *} \\
(0.0585)\end{array}$ & \\
\hline AgeAbove_80 & 0.00704 & & 0.0496 & & $0.379^{\star \star \star}$ & & $-0.463^{* * *}$ & & $-0.363^{* * *}$ & & $1.482^{* \star *}$ & & $-0.210^{* *}$ & & $-0.199^{* * *}$ & \\
\hline
\end{tabular}




\begin{tabular}{|c|c|c|c|c|c|c|c|c|c|c|c|c|c|c|c|c|}
\hline & $(0.412)$ & & $(0.0896)$ & & $(0.101)$ & & $(0.0869)$ & & $(0.0648)$ & & $(0.416)$ & & $(0.0931)$ & & $(0.0720)$ & \\
\hline \multirow[t]{2}{*}{ Married } & $0.673^{\star \star \star}$ & & 0.00117 & & 0.0350 & & -0.00700 & & $-0.0190^{\star * *}$ & & 0.0338 & & $0.176^{* \star \star}$ & & $-0.0523^{\star * *}$ & \\
\hline & $(0.127)$ & & $(0.0276)$ & & $(0.0299)$ & & $(0.0112)$ & & $(0.00668)$ & & $(0.181)$ & & $(0.0303)$ & & $(0.0193)$ & \\
\hline Reg_Partnership & $\begin{array}{l}0.591^{* *} \\
(0.271)\end{array}$ & & $\begin{array}{c}-0.00909 \\
(0.0434)\end{array}$ & & $\begin{array}{c}0.0271 \\
(0.0662)\end{array}$ & & $\begin{array}{c}0.0197 \\
(0.0181)\end{array}$ & & $\begin{array}{l}-0.0203^{* * *} \\
(0.00753)\end{array}$ & & $\begin{array}{c}0.101 \\
(0.324)\end{array}$ & & $\begin{array}{c}0.149^{* *} \\
(0.0579)\end{array}$ & & $\begin{array}{l}-0.0114 \\
(0.0249)\end{array}$ & \\
\hline Separated & $\begin{array}{l}-0.396^{*} \\
(0.225)\end{array}$ & & $\begin{array}{c}0.0206 \\
(0.0267)\end{array}$ & & $\begin{array}{l}0.118^{\star *} \\
(0.0489)\end{array}$ & & $\begin{array}{l}0.0462^{* *} \\
(0.0235)\end{array}$ & & $\begin{array}{c}-0.0193^{* * \star} \\
(0.00618)\end{array}$ & & $\begin{array}{c}-0.00258 \\
(0.238)\end{array}$ & & $\begin{array}{l}0.147^{* *} \\
(0.0570)\end{array}$ & & $\begin{array}{l}-0.0490 \\
(0.0377)\end{array}$ & \\
\hline Divorced & $\begin{array}{l}-0.356^{*} \\
(0.197)\end{array}$ & & $\begin{array}{c}0.0287 \\
(0.0319)\end{array}$ & & $\begin{array}{l}0.122^{\star * *} \\
(0.0298)\end{array}$ & & $\begin{array}{c}0.0179 \\
(0.0126)\end{array}$ & & $\begin{array}{c}-0.0223^{\star * *} \\
(0.00670)\end{array}$ & & $\begin{array}{c}0.434^{\star \star *} \\
(0.151)\end{array}$ & & $\begin{array}{l}0.154^{\star * \star} \\
(0.0348)\end{array}$ & & $\begin{array}{c}0.000384 \\
(0.0197)\end{array}$ & \\
\hline Widowed & $\begin{array}{c}0.166 \\
(0.160)\end{array}$ & & $\begin{array}{c}-0.00242 \\
(0.0274)\end{array}$ & & $\begin{array}{l}0.115^{\star * *} \\
(0.0346)\end{array}$ & & $\begin{array}{c}0.0110 \\
(0.0129)\end{array}$ & & $\begin{array}{c}-0.0111 \\
(0.00762)\end{array}$ & & $\begin{array}{c}0.109 \\
(0.192)\end{array}$ & & $\begin{array}{c}0.0346 \\
(0.0332)\end{array}$ & & $\begin{array}{c}0.0102 \\
(0.0169)\end{array}$ & \\
\hline Retired & $\begin{array}{c}2.594^{* * *} \\
(0.239)\end{array}$ & & $\begin{array}{l}-0.434^{* * *} \\
(0.0426)\end{array}$ & & $\begin{array}{c}-0.287^{\star * \star} \\
(0.0397)\end{array}$ & & $\begin{array}{l}-0.246^{\star * *} \\
(0.0423)\end{array}$ & & $\begin{array}{l}-0.103^{* * *} \\
(0.0239)\end{array}$ & & $\begin{array}{c}-3.116^{* * *} \\
(0.403)\end{array}$ & & $\begin{array}{l}0.209^{* * *} \\
(0.0311)\end{array}$ & & $\begin{array}{l}-0.354^{* * *} \\
(0.0437)\end{array}$ & \\
\hline Employed & $\begin{array}{c}2.934^{\star \star *} \\
(0.259)\end{array}$ & & $\begin{array}{c}-0.648^{\star * *} \\
(0.0395)\end{array}$ & & $\begin{array}{l}-0.508^{\star \star *} \\
(0.0350)\end{array}$ & & $\begin{array}{l}-0.236^{\star * *} \\
(0.0317)\end{array}$ & & $\begin{array}{c}-0.0769^{\star \star *} \\
(0.0175)\end{array}$ & & $\begin{array}{c}-4.273^{* * *} \\
(0.373)\end{array}$ & & $\begin{array}{l}0.261^{\star \star \star} \\
(0.0429)\end{array}$ & & $\begin{array}{c}-0.379^{* * *} \\
(0.0335)\end{array}$ & \\
\hline Homemaker & $\begin{array}{c}2.313^{\star \star *} \\
(0.230)\end{array}$ & & $\begin{array}{l}-0.478^{* * *} \\
(0.0490)\end{array}$ & & $\begin{array}{r}-0.364^{\star * *} \\
(0.0482)\end{array}$ & & $\begin{array}{l}-0.273^{\star * *} \\
(0.0418)\end{array}$ & & $\begin{array}{c}-0.0959^{* * *} \\
(0.0266)\end{array}$ & & $\begin{array}{c}-3.314^{* * *} \\
(0.395)\end{array}$ & & $\begin{array}{c}0.118^{* *} \\
(0.0499)\end{array}$ & & $\begin{array}{l}-0.343^{* * *} \\
(0.0421)\end{array}$ & \\
\hline Other_Job & $\begin{array}{c}2.027^{\star * *} \\
(0.285)\end{array}$ & & $\begin{array}{l}-0.461^{* * *} \\
(0.0453)\end{array}$ & & $\begin{array}{l}-0.341^{* * *} \\
(0.0490)\end{array}$ & & $\begin{array}{l}-0.166^{* * *} \\
(0.0392)\end{array}$ & & $\begin{array}{l}-0.0404^{*} \\
(0.0244)\end{array}$ & & $\begin{array}{c}-3.083^{* * *} \\
(0.431)\end{array}$ & & $\begin{array}{c}0.113^{* *} \\
(0.0544)\end{array}$ & & $\begin{array}{l}-0.276^{* * *} \\
(0.0407)\end{array}$ & \\
\hline N_Children & $\begin{array}{c}-0.0812^{\star *} \\
(0.0360)\end{array}$ & & $\begin{array}{l}-0.00994 \\
(0.00695)\end{array}$ & & $\begin{array}{l}-0.00813 \\
(0.00609)\end{array}$ & & $\begin{array}{c}0.00200 \\
(0.00287)\end{array}$ & & $\begin{array}{l}0.00331^{* *} \\
(0.00156)\end{array}$ & & $\begin{array}{l}-0.00766 \\
(0.0462)\end{array}$ & & $\begin{array}{l}-0.0212^{* *} \\
(0.00975)\end{array}$ & & $\begin{array}{c}0.0102^{* *} \\
(0.00435)\end{array}$ & \\
\hline N_Grandchildren & $\begin{array}{c}0.0355^{\star * *} \\
(0.0123)\end{array}$ & & $\begin{array}{c}0.00275 \\
(0.00214)\end{array}$ & & $\begin{array}{l}0.0127^{* * *} \\
(0.00279)\end{array}$ & & $\begin{array}{c}0.00181 \\
(0.00178)\end{array}$ & & $\begin{array}{c}0.00126 \\
(0.00110)\end{array}$ & & $\begin{array}{c}0.0442 \\
(0.0272)\end{array}$ & & $\begin{array}{l}0.000598 \\
(0.00449)\end{array}$ & & $\begin{array}{l}0.00387^{*} \\
(0.00206)\end{array}$ & \\
\hline Logincome & $\begin{array}{l}0.322^{\star * *} \\
(0.0478)\end{array}$ & & $\begin{array}{c}-0.0241^{* * *} \\
(0.00694)\end{array}$ & & $\begin{array}{c}0.0101 \\
(0.00755)\end{array}$ & & $\begin{array}{c}0.00753^{* * *} \\
(0.00185)\end{array}$ & & $\begin{array}{c}0.00257 \\
(0.00179)\end{array}$ & & $\begin{array}{l}0.0727^{*} \\
(0.0407)\end{array}$ & & $\begin{array}{c}0.0553^{\star * *} \\
(0.0150)\end{array}$ & & $\begin{array}{l}-0.000564 \\
(0.00416)\end{array}$ & \\
\hline $\begin{array}{l}\text { Year dummies } \\
\text { Country dummies }\end{array}$ & $\begin{array}{l}\text { YES } \\
\text { YES }\end{array}$ & & $\begin{array}{l}\text { YES } \\
\text { YES }\end{array}$ & & $\begin{array}{l}\text { YES } \\
\text { YES }\end{array}$ & & $\begin{array}{l}\text { YES } \\
\text { YES }\end{array}$ & & $\begin{array}{l}\text { YES } \\
\text { YES }\end{array}$ & & $\begin{array}{l}\text { YES } \\
\text { YES }\end{array}$ & & $\begin{array}{l}\text { YES } \\
\text { YES }\end{array}$ & & $\begin{array}{l}\text { YES } \\
\text { YES }\end{array}$ & \\
\hline Observations & 111,314 & 111,314 & 111,321 & 111,321 & 111,321 & 111,321 & 111,321 & 111,321 & 111,321 & 111,321 & 111,320 & 111,320 & 111,311 & 111,311 & 111,321 & 111,321 \\
\hline
\end{tabular}

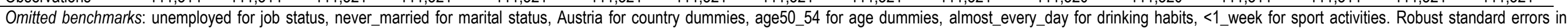
parentheses clustered at country level. ${ }^{* * *} p<0.01,{ }^{* *} p<0.05,{ }^{*} p<0.1$. 
Table A5b - Education years and specific illnesses (IV estimates)

\begin{tabular}{|c|c|c|c|c|c|c|c|c|c|c|c|c|c|c|}
\hline \multirow{3}{*}{ VARIABLES } & (1) & (2) & (3) & (4) & (5) & (6) & (7) & (8) & (9) & (10) & (11) & (12) & (13) & (14) \\
\hline & \multirow{2}{*}{\multicolumn{2}{|c|}{$\begin{array}{l}\text { longterm } \\
\text { illness }\end{array}$}} & \multirow{2}{*}{\multicolumn{2}{|c|}{ heartattack }} & \multirow{2}{*}{\multicolumn{2}{|c|}{ hypertension }} & \multirow{2}{*}{\multicolumn{2}{|c|}{ stroke }} & & & & & & \\
\hline & & & & & & & & & \multicolumn{2}{|c|}{ diabetes } & \multicolumn{2}{|c|}{ asthma } & \multicolumn{2}{|c|}{ arthritis } \\
\hline Eduyears & $\begin{array}{c}-0.0351^{* * *} \\
(0.00994)\end{array}$ & & $\begin{array}{l}-0.0160^{* *} \\
(0.00694)\end{array}$ & & $\begin{array}{c}-0.0260^{* *} \\
(0.0101)\end{array}$ & & $\begin{array}{l}-0.0160^{* *} \\
(0.00726)\end{array}$ & & $\begin{array}{c}0.00582 \\
(0.00561)\end{array}$ & & $\begin{array}{c}0.00643 \\
(0.00512)\end{array}$ & & $\begin{array}{c}-0.0412^{\star * *} \\
(0.00817)\end{array}$ & \\
\hline First_Qtr & & $\begin{array}{c}-0.0959^{* *} \\
(0.0416)\end{array}$ & & $\begin{array}{c}-0.0959^{* *} \\
(0.0481)\end{array}$ & & $\begin{array}{c}-0.0954^{* *} \\
(0.0444)\end{array}$ & & $\begin{array}{c}-0.0933^{\star *} \\
(0.0406)\end{array}$ & & $\begin{array}{l}-0.107^{\star *} \\
(0.0418)\end{array}$ & & $\begin{array}{c}-0.0921^{* *} \\
(0.0413)\end{array}$ & & $\begin{array}{c}-0.0945^{\star *} \\
(0.0388)\end{array}$ \\
\hline Age & & $\begin{array}{l}-0.124^{* *} \\
(0.0626)\end{array}$ & & $\begin{array}{l}-0.112^{*} \\
(0.0623)\end{array}$ & & $\begin{array}{l}-0.127^{*} \\
(0.0657)\end{array}$ & & $\begin{array}{c}-0.110^{*} \\
(0.0611)\end{array}$ & & $\begin{array}{l}-0.108^{*} \\
(0.0646)\end{array}$ & & $\begin{array}{c}-0.112^{*} \\
(0.0618)\end{array}$ & & $\begin{array}{l}-0.133^{\star *} \\
(0.0635)\end{array}$ \\
\hline $\mathrm{Age}^{2}$ & & $\begin{array}{c}0.000155 \\
(0.000410)\end{array}$ & & $\begin{array}{c}6.45 \mathrm{e}-05 \\
(0.000407)\end{array}$ & & $\begin{array}{c}0.000180 \\
(0.000437)\end{array}$ & & $\begin{array}{c}5.56 \mathrm{e}-05 \\
(0.000399)\end{array}$ & & $\begin{array}{c}3.74 \mathrm{e}-05 \\
(0.000427)\end{array}$ & & $\begin{array}{c}6.47 \mathrm{e}-05 \\
(0.000404)\end{array}$ & & $\begin{array}{c}0.000222 \\
(0.000417)\end{array}$ \\
\hline Female & $\begin{array}{l}-0.0168 \\
(0.0111)\end{array}$ & $\begin{array}{c}-0.786^{* * *} \\
(0.119)\end{array}$ & $\begin{array}{c}-0.0723^{\star * *} \\
(0.00733)\end{array}$ & $\begin{array}{c}-0.784^{* * *} \\
(0.119)\end{array}$ & $\begin{array}{c}0.00446 \\
(0.00962)\end{array}$ & $\begin{array}{c}-0.787^{\star * *} \\
(0.119)\end{array}$ & $\begin{array}{c}-0.0284^{* * *} \\
(0.00660)\end{array}$ & $\begin{array}{c}-0.784^{* * *} \\
(0.119)\end{array}$ & $\begin{array}{c}-0.0248^{* * *} \\
(0.00698)\end{array}$ & $\begin{array}{c}-0.783^{\star * *} \\
(0.119)\end{array}$ & $\begin{array}{c}0.00718^{*} \\
(0.00393)\end{array}$ & $\begin{array}{c}-0.784^{\star * *} \\
(0.119)\end{array}$ & $\begin{array}{c}0.0765^{\star * *} \\
(0.0124)\end{array}$ & $\begin{array}{c}-0.788^{\star * *} \\
(0.119)\end{array}$ \\
\hline Age55_59 & $\begin{array}{l}0.0215^{\star} \\
(0.0125)\end{array}$ & & $\begin{array}{c}-0.000798 \\
(0.00373)\end{array}$ & & $\begin{array}{c}0.0490^{\star * *} \\
(0.0119)\end{array}$ & & $\begin{array}{c}-0.00599 \\
(0.00433)\end{array}$ & & $\begin{array}{l}0.0197^{\star * *} \\
(0.00360)\end{array}$ & & $\begin{array}{c}-0.000345 \\
(0.00328)\end{array}$ & & $\begin{array}{l}0.0253^{* * *} \\
(0.00743)\end{array}$ & \\
\hline Age60_64 & $\begin{array}{l}0.00662 \\
(0.0180)\end{array}$ & & $\begin{array}{c}0.00509 \\
(0.00796)\end{array}$ & & $\begin{array}{c}0.0869^{\star * *} \\
(0.0194)\end{array}$ & & $\begin{array}{c}-0.0124 \\
(0.00754)\end{array}$ & & $\begin{array}{l}0.0340^{\star * *} \\
(0.00518)\end{array}$ & & $\begin{array}{c}0.00242 \\
(0.00584)\end{array}$ & & $\begin{array}{l}0.0287^{* * *} \\
(0.00963)\end{array}$ & \\
\hline Age65_69 & $\begin{array}{c}0.0110 \\
(0.0209)\end{array}$ & & $\begin{array}{c}0.0179^{*} \\
(0.00956)\end{array}$ & & $\begin{array}{l}0.120^{\star * *} \\
(0.0257)\end{array}$ & & $\begin{array}{c}-0.0133 \\
(0.0116)\end{array}$ & & $\begin{array}{c}0.0598^{* * *} \\
(0.0101)\end{array}$ & & $\begin{array}{c}0.00337 \\
(0.00830)\end{array}$ & & $\begin{array}{l}0.0321^{* *} \\
(0.0139)\end{array}$ & \\
\hline Age70_74 & $\begin{array}{c}0.0204 \\
(0.0252)\end{array}$ & & $\begin{array}{c}0.0486^{* * *} \\
(0.0125)\end{array}$ & & $\begin{array}{l}0.144^{* * *} \\
(0.0296)\end{array}$ & & $\begin{array}{l}-0.0139 \\
(0.0147)\end{array}$ & & $\begin{array}{c}0.0689^{* * *} \\
(0.0110)\end{array}$ & & $\begin{array}{c}0.0104 \\
(0.0114)\end{array}$ & & $\begin{array}{l}0.0269^{*} \\
(0.0158)\end{array}$ & \\
\hline Age75_79 & $\begin{array}{c}0.0270 \\
(0.0307)\end{array}$ & & $\begin{array}{c}0.0841^{* * *} \\
(0.0176)\end{array}$ & & $\begin{array}{l}0.141^{* * *} \\
(0.0330)\end{array}$ & & $\begin{array}{c}-0.00326 \\
(0.0182)\end{array}$ & & $\begin{array}{c}0.0762^{\star * *} \\
(0.0145)\end{array}$ & & $\begin{array}{c}0.0126 \\
(0.0133)\end{array}$ & & $\begin{array}{l}0.0325^{*} \\
(0.0192)\end{array}$ & \\
\hline AgeAbove_80 & $\begin{array}{c}0.0224 \\
(0.0366)\end{array}$ & & $\begin{array}{c}0.0973^{* * *} \\
(0.0171)\end{array}$ & & $\begin{array}{l}0.0985^{\star *} \\
(0.0400)\end{array}$ & & $\begin{array}{c}-0.00402 \\
(0.0240)\end{array}$ & & $\begin{array}{c}0.0686^{\star * *} \\
(0.0173)\end{array}$ & & $\begin{array}{c}0.0148 \\
(0.0173)\end{array}$ & & $\begin{array}{c}0.0312 \\
(0.0200)\end{array}$ & \\
\hline Married & $\begin{array}{c}-0.00702 \\
(0.00787)\end{array}$ & & $\begin{array}{c}0.0122^{*} \\
(0.00737)\end{array}$ & & $\begin{array}{c}0.0140 \\
(0.00949)\end{array}$ & & $\begin{array}{c}0.00398 \\
(0.00318)\end{array}$ & & $\begin{array}{l}-0.00822 \\
(0.00596)\end{array}$ & & $\begin{array}{c}-0.00202 \\
(0.00315)\end{array}$ & & $\begin{array}{c}-0.00304 \\
(0.00867)\end{array}$ & \\
\hline Reg_Partnership & $\begin{array}{c}-0.00669 \\
(0.0177)\end{array}$ & & $\begin{array}{l}0.00881 \\
(0.0164)\end{array}$ & & $\begin{array}{l}-0.0123 \\
(0.0159)\end{array}$ & & $\begin{array}{c}0.00290 \\
(0.00478)\end{array}$ & & $\begin{array}{r}-0.00829 \\
(0.0109)\end{array}$ & & $\begin{array}{c}0.00469 \\
(0.00737)\end{array}$ & & $\begin{array}{c}-0.000612 \\
(0.0137)\end{array}$ & \\
\hline Separated & $\begin{array}{l}0.00746 \\
(0.0189)\end{array}$ & & $\begin{array}{l}0.00813 \\
(0.0134)\end{array}$ & & $\begin{array}{l}0.00871 \\
(0.0155)\end{array}$ & & $\begin{array}{l}0.0236^{\star * *} \\
(0.00733)\end{array}$ & & $\begin{array}{c}0.0178 \\
(0.0131)\end{array}$ & & $\begin{array}{c}-0.00235 \\
(0.00444)\end{array}$ & & $\begin{array}{l}0.00866 \\
(0.0101)\end{array}$ & \\
\hline Divorced & $\begin{array}{c}0.0330^{\star * *} \\
(0.0112)\end{array}$ & & $\begin{array}{l}0.0237^{\star \star *} \\
(0.00901)\end{array}$ & & $\begin{array}{c}-0.00270 \\
(0.00797)\end{array}$ & & $\begin{array}{c}0.00615 \\
(0.00393)\end{array}$ & & $\begin{array}{l}-0.00377 \\
(0.00658)\end{array}$ & & $\begin{array}{c}0.00874^{\star * *} \\
(0.00297)\end{array}$ & & $\begin{array}{l}0.0290^{* * *} \\
(0.00907)\end{array}$ & \\
\hline Widowed & $\begin{array}{l}0.000576 \\
(0.00962)\end{array}$ & & $\begin{array}{c}0.0160^{* *} \\
(0.00649)\end{array}$ & & $\begin{array}{c}0.0442^{\star * *} \\
(0.0114)\end{array}$ & & $\begin{array}{c}0.00121 \\
(0.00385)\end{array}$ & & $\begin{array}{c}0.00585 \\
(0.00729)\end{array}$ & & $\begin{array}{c}0.00123 \\
(0.00355)\end{array}$ & & $\begin{array}{c}0.0172^{*} \\
(0.00989)\end{array}$ & \\
\hline Retired & $\begin{array}{c}-0.182^{* \star \star} \\
(0.0196)\end{array}$ & & $\begin{array}{c}-0.0375^{\star * \star} \\
(0.00743)\end{array}$ & & $\begin{array}{c}-0.0277^{* * *} \\
(0.00877)\end{array}$ & & $\begin{array}{c}-0.0294^{* * *} \\
(0.00497)\end{array}$ & & $\begin{array}{c}-0.0285^{\star \star *} \\
(0.00693)\end{array}$ & & $\begin{array}{c}-0.00891^{* *} \\
(0.00438)\end{array}$ & & $\begin{array}{c}-0.0744^{* * *} \\
(0.00640)\end{array}$ & \\
\hline Employed & $\begin{array}{c}-0.277^{* * *} \\
(0.0176)\end{array}$ & & $\begin{array}{c}-0.0677^{* * *} \\
(0.00723)\end{array}$ & & $\begin{array}{c}-0.0551^{* * *} \\
(0.0113)\end{array}$ & & $\begin{array}{c}-0.0424^{* * *} \\
(0.00449)\end{array}$ & & $\begin{array}{c}-0.0489^{* * *} \\
(0.00666)\end{array}$ & & $\begin{array}{l}-0.0161^{\star * *} \\
(0.00468)\end{array}$ & & $\begin{array}{c}-0.0980^{* * *} \\
(0.00778)\end{array}$ & \\
\hline Homemaker & $\begin{array}{l}-0.220^{* * *} \\
(0.0203)\end{array}$ & & $\begin{array}{c}-0.0544^{* * *} \\
(0.00831)\end{array}$ & & $\begin{array}{c}-0.0272^{* *} \\
(0.0110)\end{array}$ & & $\begin{array}{c}-0.0362^{* * *} \\
(0.00625)\end{array}$ & & $\begin{array}{c}-0.0287^{* * *} \\
(0.00844)\end{array}$ & & $\begin{array}{l}-0.0115^{\star * *} \\
(0.00423)\end{array}$ & & $\begin{array}{c}-0.0699^{\star * *} \\
(0.0138)\end{array}$ & \\
\hline Other_Job & $\begin{array}{c}-0.178^{* * *} \\
(0.0302)\end{array}$ & & $\begin{array}{c}-0.0382^{* * *} \\
(0.0107)\end{array}$ & & $\begin{array}{c}-0.0409^{* *} \\
(0.0181)\end{array}$ & & $\begin{array}{c}-0.0260^{* * *} \\
(0.00710)\end{array}$ & & $\begin{array}{c}-0.0342^{* *} \\
(0.0159)\end{array}$ & & $\begin{array}{c}-0.0225^{\star * *} \\
(0.00741)\end{array}$ & & $\begin{array}{c}-0.0620^{* * *} \\
(0.0124)\end{array}$ & \\
\hline N_Children & $\begin{array}{c}-0.00231 \\
(0.00225)\end{array}$ & & $\begin{array}{l}0.000458 \\
(0.00137)\end{array}$ & & $\begin{array}{c}-0.00123 \\
(0.00139)\end{array}$ & & $\begin{array}{c}0.00142 \\
(0.000985)\end{array}$ & & $\begin{array}{c}0.00178 \\
(0.00138)\end{array}$ & & $\begin{array}{c}0.000765 \\
(0.000512)\end{array}$ & & $\begin{array}{c}0.00171 \\
(0.00205)\end{array}$ & \\
\hline N_Grandchildren & $\begin{array}{l}0.00371^{* * *} \\
(0.000623)\end{array}$ & & $\begin{array}{l}0.00254^{* * *} \\
(0.000630)\end{array}$ & & $\begin{array}{l}0.00199^{* * *} \\
(0.000651)\end{array}$ & & $\begin{array}{c}-9.88 \mathrm{e}-06 \\
(0.000454)\end{array}$ & & $\begin{array}{c}0.00149^{* *} \\
(0.000623)\end{array}$ & & $\begin{array}{c}0.000691^{* * *} \\
(0.000254)\end{array}$ & & $\begin{array}{c}0.00178^{* *} \\
(0.000900)\end{array}$ & \\
\hline
\end{tabular}




\begin{tabular}{|c|c|c|c|c|c|c|c|c|c|c|c|c|c|c|}
\hline Logincome & $\begin{array}{l}0.00563^{* *} \\
(0.00269)\end{array}$ & & $\begin{array}{c}0.00118 \\
(0.00132)\end{array}$ & & $\begin{array}{c}-0.00119 \\
(0.00185)\end{array}$ & & $\begin{array}{c}0.00149^{* *} \\
(0.000635)\end{array}$ & & $\begin{array}{r}-0.000877 \\
(0.00120)\end{array}$ & & $\begin{array}{l}0.00218^{* *} \\
(0.00106)\end{array}$ & & $\begin{array}{l}0.000211 \\
(0.00150)\end{array}$ & \\
\hline \multicolumn{15}{|l|}{ DRINKING } \\
\hline 5or6days_week & $\begin{array}{c}-0.0243^{* * *} \\
(0.00713)\end{array}$ & & $\begin{array}{c}-0.0271^{* * *} \\
(0.00731)\end{array}$ & & $\begin{array}{c}-0.00222 \\
(0.00731)\end{array}$ & & $\begin{array}{c}-0.0182^{* * *} \\
(0.00476)\end{array}$ & & $\begin{array}{c}-0.0255^{\star * *} \\
(0.00301)\end{array}$ & & $\begin{array}{c}-0.00400^{* *} \\
(0.00163)\end{array}$ & & $\begin{array}{l}0.000732 \\
(0.00598)\end{array}$ & \\
\hline 3or4days_week & $\begin{array}{c}-0.0471^{* * *} \\
(0.00827)\end{array}$ & & $\begin{array}{c}-0.0301^{* * *} \\
(0.00713)\end{array}$ & & $\begin{array}{c}-0.00982 \\
(0.00729)\end{array}$ & & $\begin{array}{c}-0.0197^{* * *} \\
(0.00423)\end{array}$ & & $\begin{array}{c}-0.0361^{* * *} \\
(0.00374)\end{array}$ & & $\begin{array}{c}-0.00457^{* *} \\
(0.00219)\end{array}$ & & $\begin{array}{c}0.00567 \\
(0.00577)\end{array}$ & \\
\hline 1or2_week & $\begin{array}{l}-0.0677^{* * *} \\
(0.00667)\end{array}$ & & $\begin{array}{l}-0.0347^{* * *} \\
(0.00614)\end{array}$ & & $\begin{array}{c}-0.0179^{* * *} \\
(0.00635)\end{array}$ & & $\begin{array}{c}-0.0215^{\star * *} \\
(0.00453)\end{array}$ & & $\begin{array}{l}-0.0457^{* * *} \\
(0.00478)\end{array}$ & & $\begin{array}{c}-0.00439^{* * *} \\
(0.00160)\end{array}$ & & $\begin{array}{c}0.00181 \\
(0.00621)\end{array}$ & \\
\hline 1or2_month & $\begin{array}{c}-0.0585^{\star * *} \\
(0.0101)\end{array}$ & & $\begin{array}{l}-0.0340^{* * *} \\
(0.00785)\end{array}$ & & $\begin{array}{l}-0.0162^{* * *} \\
(0.00549)\end{array}$ & & $\begin{array}{c}-0.0246^{* * *} \\
(0.00429)\end{array}$ & & $\begin{array}{l}-0.0495^{\star * *} \\
(0.00552)\end{array}$ & & $\begin{array}{c}-0.00516^{* *} \\
(0.00218)\end{array}$ & & $\begin{array}{c}0.00197 \\
(0.00791)\end{array}$ & \\
\hline$<1$ _month & $\begin{array}{c}-0.0769^{* * *} \\
(0.0113)\end{array}$ & & $\begin{array}{l}-0.0390^{* * *} \\
(0.00657)\end{array}$ & & $\begin{array}{c}-0.0205^{* * *} \\
(0.00790)\end{array}$ & & $\begin{array}{c}-0.0234^{* * *} \\
(0.00365)\end{array}$ & & $\begin{array}{l}-0.0624^{* * *} \\
(0.00573)\end{array}$ & & $\begin{array}{c}-0.00603^{* *} \\
(0.00245)\end{array}$ & & $\begin{array}{l}-0.00215 \\
(0.00825)\end{array}$ & \\
\hline 0_in_3months & $\begin{array}{l}-0.0623^{* * *} \\
(0.00731)\end{array}$ & & $\begin{array}{c}-0.0353^{* * *} \\
(0.00559)\end{array}$ & & $\begin{array}{l}-0.00226 \\
(0.00680)\end{array}$ & & $\begin{array}{l}-0.0194^{* * *} \\
(0.00347)\end{array}$ & & $\begin{array}{c}-0.0543^{* * *} \\
(0.00648)\end{array}$ & & $\begin{array}{l}-0.00156^{\star *} \\
(0.000766)\end{array}$ & & $\begin{array}{c}0.00528 \\
(0.00718)\end{array}$ & \\
\hline \multicolumn{15}{|l|}{ SPORT } \\
\hline 1_week & $\begin{array}{c}0.00925 \\
(0.00638)\end{array}$ & & $\begin{array}{c}0.00428 \\
(0.00334)\end{array}$ & & $\begin{array}{l}0.0189^{* * *} \\
(0.00646)\end{array}$ & & $\begin{array}{l}0.000105 \\
(0.00123)\end{array}$ & & $\begin{array}{l}0.00633^{*} \\
(0.00333)\end{array}$ & & $\begin{array}{l}0.000497 \\
(0.00161)\end{array}$ & & $\begin{array}{c}0.00509 \\
(0.00409)\end{array}$ & \\
\hline 1or3_month & $\begin{array}{l}0.0461^{* * *} \\
(0.00789)\end{array}$ & & $\begin{array}{l}0.0200^{* * *} \\
(0.00449)\end{array}$ & & $\begin{array}{l}0.0270^{\star * *} \\
(0.00805)\end{array}$ & & $\begin{array}{c}0.00226 \\
(0.00202)\end{array}$ & & $\begin{array}{l}0.0137^{* * *} \\
(0.00388)\end{array}$ & & $\begin{array}{c}0.00286 \\
(0.00184)\end{array}$ & & $\begin{array}{c}0.00739 \\
(0.00559)\end{array}$ & \\
\hline Hardlyever_never & $\begin{array}{c}0.159 * * * \\
(0.00993)\end{array}$ & & $\begin{array}{l}0.0672^{* * *} \\
(0.00647)\end{array}$ & & $\begin{array}{l}0.0650^{* * *} \\
(0.00809)\end{array}$ & & $\begin{array}{l}0.0298^{* * *} \\
(0.00513)\end{array}$ & & $\begin{array}{l}0.0460^{* * *} \\
(0.00444)\end{array}$ & & $\begin{array}{l}0.0124^{* * *} \\
(0.00216)\end{array}$ & & $\begin{array}{l}0.0617^{* * *} \\
(0.00714)\end{array}$ & \\
\hline Smoking & $\begin{array}{c}-0.00868 \\
(0.00541)\end{array}$ & & $\begin{array}{c}-0.0171^{* * *} \\
(0.00270)\end{array}$ & & $\begin{array}{c}-0.0389^{\star * \star} \\
(0.00544)\end{array}$ & & $\begin{array}{l}-5.10 \mathrm{e}-05 \\
(0.00180)\end{array}$ & & $\begin{array}{c}-0.00728^{\star *} \\
(0.00306)\end{array}$ & & $\begin{array}{l}-0.00297 \\
(0.00191)\end{array}$ & & $\begin{array}{l}-0.00239 \\
(0.00402)\end{array}$ & \\
\hline Overweight_Obese & $\begin{array}{l}0.0715^{\star \star \star} \\
(0.00489)\end{array}$ & & $\begin{array}{l}0.0216^{\star * *} \\
(0.00404)\end{array}$ & & $\begin{array}{c}0.164^{\star * *} \\
(0.00702)\end{array}$ & & $\begin{array}{l}0.000859 \\
(0.00120)\end{array}$ & & $\begin{array}{l}0.0700^{\star * \star} \\
(0.00341)\end{array}$ & & $\begin{array}{c}0.00469^{* * *} \\
(0.00169)\end{array}$ & & $\begin{array}{l}0.0476^{\star * \star} \\
(0.00491)\end{array}$ & \\
\hline $\begin{array}{l}\text { Year dummies } \\
\text { Country dummies }\end{array}$ & $\begin{array}{l}\text { YES } \\
\text { YES }\end{array}$ & & $\begin{array}{l}\text { YES } \\
\text { YES }\end{array}$ & & $\begin{array}{l}\text { YES } \\
\text { YES }\end{array}$ & & $\begin{array}{l}\text { YES } \\
\text { YES }\end{array}$ & & $\begin{array}{l}\text { YES } \\
\text { YES }\end{array}$ & & $\begin{array}{l}\text { YES } \\
\text { YES }\end{array}$ & & $\begin{array}{l}\text { YES } \\
\text { YES }\end{array}$ & \\
\hline Observations & 111,321 & 111,321 & 111,321 & 111,321 & 111,321 & 111,321 & 111,321 & 111,321 & 111,321 & 111,321 & 111,321 & 111,321 & 111,321 & 111,321 \\
\hline
\end{tabular}

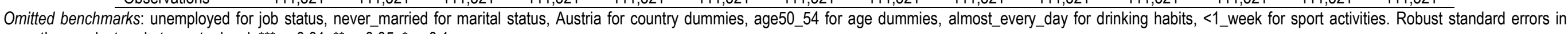
parentheses clustered at country level. ${ }^{* * *} p<0.01,{ }^{* *} p<0.05,{ }^{*} p<0.1$.

Table A5b - Education years and specific illnesses (IV estimates) - continued

\begin{tabular}{|c|c|c|c|c|c|c|c|c|c|c|c|c|}
\hline \multirow[b]{3}{*}{ VARIABLES } & (15) & (16) & (17) & (18) & (19) & (20) & (21) & (22) & (23) & (24) & (25) & (26) \\
\hline & \multicolumn{2}{|c|}{ cancer } & \multicolumn{2}{|c|}{ ulcer } & \multicolumn{2}{|c|}{ parkinson } & \multicolumn{2}{|c|}{ cataracts } & \multicolumn{2}{|c|}{ fracture } & \multicolumn{2}{|c|}{ none } \\
\hline & 2nd stage & 1st stage & 2nd stage & 1st stage & 2nd stage & 1st stage & 2nd stage & 1st stage & 2nd stage & 1st stage & 2nd stage & 1 st stage \\
\hline Eduyears & $\begin{array}{l}-0.00535^{*} \\
(0.00316)\end{array}$ & & $\begin{array}{c}9.75 e-05 \\
(0.00405)\end{array}$ & & $\begin{array}{l}-0.0111^{*} \\
(0.00664)\end{array}$ & & $\begin{array}{c}-0.0388^{* * *} \\
(0.00718)\end{array}$ & & $\begin{array}{c}-0.0125^{\star * *} \\
(0.00308)\end{array}$ & & $\begin{array}{c}0.0840^{* * *} \\
(0.0145)\end{array}$ & \\
\hline First_Qtr & & $\begin{array}{c}-0.0998^{* *} \\
(0.0433)\end{array}$ & & $\begin{array}{c}-0.108^{* * *} \\
(0.0415)\end{array}$ & & $\begin{array}{c}-0.109^{* * *} \\
(0.0391)\end{array}$ & & $\begin{array}{c}-0.0917^{\star *} \\
(0.0426)\end{array}$ & & $\begin{array}{c}-0.103^{\star * *} \\
(0.0382)\end{array}$ & & $\begin{array}{l}-0.0675^{*} \\
(0.0376)\end{array}$ \\
\hline Age & & $\begin{array}{l}-0.115^{\star} \\
(0.0629)\end{array}$ & & $\begin{array}{l}-0.113^{*} \\
(0.0626)\end{array}$ & & $\begin{array}{l}-0.0991 \\
(0.0610)\end{array}$ & & $\begin{array}{c}-0.0916 \\
(0.0601)\end{array}$ & & $\begin{array}{c}-0.0995 \\
(0.0617)\end{array}$ & & $\begin{array}{c}-0.175^{\star * \star} \\
(0.0581)\end{array}$ \\
\hline $\mathrm{Age}^{2}$ & & $\begin{array}{c}8.61 \mathrm{e}-05 \\
(0.000413)\end{array}$ & & $\begin{array}{c}7.33 e-05 \\
(0.000411)\end{array}$ & & $\begin{array}{c}-2.32 \mathrm{e}-05 \\
(0.000401)\end{array}$ & & $\begin{array}{c}-8.68 \mathrm{e}-05 \\
(0.000390)\end{array}$ & & $\begin{array}{c}-2.80 \mathrm{e}-05 \\
(0.000402)\end{array}$ & & $\begin{array}{c}0.000538 \\
(0.000382)\end{array}$ \\
\hline Female & $\begin{array}{l}0.000142 \\
(0.00207)\end{array}$ & $\begin{array}{c}-0.785^{\text {*** }} \\
(0.119)\end{array}$ & $\begin{array}{c}-0.00944^{* * *} \\
(0.00338)\end{array}$ & $\begin{array}{c}-0.784^{* * *} \\
(0.119)\end{array}$ & $\begin{array}{l}-0.0115^{* *} \\
(0.00552)\end{array}$ & $\begin{array}{c}-0.781^{* * *} \\
(0.117)\end{array}$ & $\begin{array}{c}-0.00796 \\
(0.00721)\end{array}$ & $\begin{array}{c}-0.780^{\star * \star} \\
(0.119)\end{array}$ & $\begin{array}{c}-0.00677^{* *} \\
(0.00311)\end{array}$ & $\begin{array}{c}-0.782^{\star * *} \\
(0.119)\end{array}$ & $\begin{array}{c}0.0457^{\star * *} \\
(0.0144)\end{array}$ & $\begin{array}{c}-0.794^{* * *} \\
(0.119)\end{array}$ \\
\hline
\end{tabular}




\begin{tabular}{|c|c|c|c|c|c|c|}
\hline Age55_59 & $\begin{array}{l}0.000843 \\
(0.00379)\end{array}$ & $\begin{array}{l}-0.000775 \\
(0.00439)\end{array}$ & $\begin{array}{l}-0.00603^{*} \\
(0.00347)\end{array}$ & $\begin{array}{c}-0.0161^{\star * *} \\
(0.00262)\end{array}$ & $\begin{array}{c}-0.00649^{\star * *} \\
(0.00190)\end{array}$ & $\begin{array}{c}-0.0321^{* *} \\
(0.0149)\end{array}$ \\
\hline Age60_64 & 0.00196 & 0.000868 & -0.00970 & $-0.0205^{\star * *}$ & $-0.0106^{\star * \star}$ & -0.0314 \\
\hline & $(0.00512)$ & $(0.00511)$ & $(0.00637)$ & $(0.00573)$ & $(0.00283)$ & $(0.0225)$ \\
\hline Age65 69 & 0.000946 & 0.00118 & -0.0133 & $-0.0157^{\star *}$ & $-0.0145^{\star \star *}$ & -0.0244 \\
\hline & $(0.00620)$ & $(0.00664)$ & $(0.00918)$ & $(0.00729)$ & $(0.00377)$ & $(0.0269)$ \\
\hline Age70_74 & $\begin{array}{c}0.00851 \\
(0.00869)\end{array}$ & $\begin{array}{c}0.00338 \\
(0.00948)\end{array}$ & $\begin{array}{l}-0.0166 \\
(0.0122)\end{array}$ & $\begin{array}{c}0.00665 \\
(0.00861)\end{array}$ & $\begin{array}{l}-0.0152^{* * *} \\
(0.00493)\end{array}$ & $\begin{array}{l}-0.0210 \\
(0.0317)\end{array}$ \\
\hline Age75_79 & $\begin{array}{l}0.00515 \\
(0.0101)\end{array}$ & $\begin{array}{l}0.00660 \\
(0.0109)\end{array}$ & $\begin{array}{l}-0.0194 \\
(0.0163)\end{array}$ & $\begin{array}{c}0.0465^{\star \star *} \\
(0.0126)\end{array}$ & $\begin{array}{l}-0.00953 \\
(0.00657)\end{array}$ & $\begin{array}{c}-0.00229 \\
(0.0363)\end{array}$ \\
\hline AgeAbove_80 & $\begin{array}{c}0.000507 \\
(0.0132)\end{array}$ & $\begin{array}{l}0.00603 \\
(0.0139)\end{array}$ & $\begin{array}{l}-0.0212 \\
(0.0196)\end{array}$ & $\begin{array}{c}0.0755^{\star * *} \\
(0.0154)\end{array}$ & $\begin{array}{c}0.00129 \\
(0.00822)\end{array}$ & $\begin{array}{c}0.0336 \\
(0.0374)\end{array}$ \\
\hline Married & $\begin{array}{l}0.00575^{\star *} \\
(0.00289)\end{array}$ & $\begin{array}{c}-0.000462 \\
(0.00359)\end{array}$ & $\begin{array}{l}0.000516 \\
(0.00154)\end{array}$ & $\begin{array}{c}-0.00873^{* *} \\
(0.00378)\end{array}$ & $\begin{array}{l}-0.00174 \\
(0.00243)\end{array}$ & $\begin{array}{c}0.00343 \\
(0.00654)\end{array}$ \\
\hline Reg_Partnership & $\begin{array}{l}-0.00352 \\
(0.00633)\end{array}$ & $\begin{array}{c}0.00231 \\
(0.00637)\end{array}$ & $\begin{array}{c}0.00490 \\
(0.00307)\end{array}$ & $\begin{array}{l}-0.00221 \\
(0.00836)\end{array}$ & $\begin{array}{l}-0.00103 \\
(0.00341)\end{array}$ & $\begin{array}{c}-0.00222 \\
(0.0151)\end{array}$ \\
\hline Separated & $\begin{array}{c}0.00725 \\
(0.00774)\end{array}$ & $\begin{array}{c}0.00946 \\
(0.00813)\end{array}$ & $\begin{array}{c}0.00319 \\
(0.00327)\end{array}$ & $\begin{array}{c}0.00194 \\
(0.00685)\end{array}$ & $\begin{array}{r}-0.000907 \\
(0.00428)\end{array}$ & $\begin{array}{l}-0.0131 \\
(0.0194)\end{array}$ \\
\hline Divorced & $\begin{array}{c}0.00967^{* * *} \\
(0.00332)\end{array}$ & $\begin{array}{l}0.0143^{* * *} \\
(0.00406)\end{array}$ & $\begin{array}{l}-0.00107 \\
(0.00170)\end{array}$ & $\begin{array}{c}0.00188 \\
(0.00391)\end{array}$ & $\begin{array}{c}0.00199 \\
(0.00289)\end{array}$ & $\begin{array}{c}-0.0215^{\star * *} \\
(0.00827)\end{array}$ \\
\hline Widowed & $\begin{array}{r}-0.000739 \\
(0.00325)\end{array}$ & $\begin{array}{r}-0.000304 \\
(0.00552)\end{array}$ & $\begin{array}{r}-0.000654 \\
(0.00168)\end{array}$ & $\begin{array}{c}0.00880 \\
(0.00568)\end{array}$ & $\begin{array}{l}0.00535^{\star *} \\
(0.00239)\end{array}$ & $\begin{array}{l}-0.00948 \\
(0.00779)\end{array}$ \\
\hline Retired & $\begin{array}{c}-0.0131^{* * *} \\
(0.00482)\end{array}$ & $\begin{array}{l}-0.0193^{\star \star} \\
(0.00753)\end{array}$ & $\begin{array}{l}-0.00288 \\
(0.00224)\end{array}$ & $\begin{array}{l}-0.0104^{* *} \\
(0.00421)\end{array}$ & $\begin{array}{c}-0.0138^{* * *} \\
(0.00286)\end{array}$ & $\begin{array}{c}0.0957^{* * *} \\
(0.0105)\end{array}$ \\
\hline Employed & $\begin{array}{c}-0.0364^{* \star *} \\
(0.00365)\end{array}$ & $\begin{array}{c}-0.0323^{\star \star \star} \\
(0.00675)\end{array}$ & $\begin{array}{c}-0.00396^{* \star *} \\
(0.00141)\end{array}$ & $\begin{array}{l}-0.0208^{* * *} \\
(0.00250)\end{array}$ & $\begin{array}{l}-0.0148^{* \star *} \\
(0.00238)\end{array}$ & $\begin{array}{l}0.178^{* * *} \\
(0.0120)\end{array}$ \\
\hline Homemaker & $\begin{array}{c}-0.0244^{* * *} \\
(0.00470)\end{array}$ & $\begin{array}{c}-0.0264^{* * *} \\
(0.00801)\end{array}$ & $\begin{array}{c}-0.00533^{* *} \\
(0.00209)\end{array}$ & $\begin{array}{c}-0.0160^{* * *} \\
(0.00437)\end{array}$ & $\begin{array}{c}-0.0113^{* * *} \\
(0.00213)\end{array}$ & $\begin{array}{l}0.110^{* * *} \\
(0.0121)\end{array}$ \\
\hline Other_Job & $\begin{array}{l}-0.0189^{* *} \\
(0.00878)\end{array}$ & $\begin{array}{c}-0.0296 * * * \\
(0.00752)\end{array}$ & $\begin{array}{l}-0.00368 \\
(0.00304)\end{array}$ & $\begin{array}{l}-0.0189^{* *} \\
(0.00842)\end{array}$ & $\begin{array}{c}-0.0129^{* * *} \\
(0.00419)\end{array}$ & $\begin{array}{l}0.104^{* * *} \\
(0.0124)\end{array}$ \\
\hline N_Children & $\begin{array}{l}-0.00216^{* * *} \\
(0.000752)\end{array}$ & $\begin{array}{l}-0.000632 \\
(0.000995)\end{array}$ & $\begin{array}{c}0.000272 \\
(0.000300)\end{array}$ & $\begin{array}{l}-0.00143 \\
(0.00128)\end{array}$ & $\begin{array}{l}-0.000242 \\
(0.000560)\end{array}$ & $\begin{array}{l}0.00368^{*} \\
(0.00199)\end{array}$ \\
\hline N_Grandchildren & $\begin{array}{c}3.65 \mathrm{e}-05 \\
(0.000308)\end{array}$ & $\begin{array}{l}0.000817^{*} \\
(0.000460)\end{array}$ & $\begin{array}{c}-4.85 e-05 \\
(0.000190)\end{array}$ & $\begin{array}{c}6.49 \mathrm{e}-05 \\
(0.000457)\end{array}$ & $\begin{array}{c}1.07 \mathrm{e}-05 \\
(0.000225)\end{array}$ & $\begin{array}{c}-0.00315^{\star * *} \\
(0.000718)\end{array}$ \\
\hline Logincome & $\begin{array}{l}0.00490^{* * *} \\
(0.00110)\end{array}$ & $\begin{array}{l}-5.69 e-05 \\
(0.00110)\end{array}$ & $\begin{array}{c}0.000502^{* * *} \\
(0.000195)\end{array}$ & $\begin{array}{l}0.00257^{*} \\
(0.00153)\end{array}$ & $\begin{array}{c}0.000968^{*} \\
(0.000563)\end{array}$ & $\begin{array}{c}-0.00670^{* * *} \\
(0.00236)\end{array}$ \\
\hline DRINKING & & & & & & \\
\hline 5or6days_week & $\begin{array}{c}-0.00632^{* * *} \\
(0.00203)\end{array}$ & $\begin{array}{l}-0.00352 \\
(0.00413)\end{array}$ & $\begin{array}{c}-0.00353^{* * *} \\
(0.000872)\end{array}$ & $\begin{array}{c}0.00467 \\
(0.00439)\end{array}$ & $\begin{array}{l}-0.00110 \\
(0.00172)\end{array}$ & $\begin{array}{l}-0.00525 \\
(0.00719)\end{array}$ \\
\hline 3or4days_week & $\begin{array}{c}-0.00476^{\star *} \\
(0.00216)\end{array}$ & $\begin{array}{l}-0.00319 \\
(0.00362)\end{array}$ & $\begin{array}{c}-0.00372^{* * *} \\
(0.00103)\end{array}$ & $\begin{array}{c}0.00501 \\
(0.00377)\end{array}$ & $\begin{array}{l}-0.00207 \\
(0.00137)\end{array}$ & $\begin{array}{c}0.00641 \\
(0.00625)\end{array}$ \\
\hline 1or2_week & $\begin{array}{c}-0.00693^{* * *} \\
(0.00227)\end{array}$ & $\begin{array}{c}-0.00591 \\
(0.00454)\end{array}$ & $\begin{array}{c}-0.00459^{* * *} \\
(0.00103)\end{array}$ & $\begin{array}{c}0.00337 \\
(0.00397)\end{array}$ & $\begin{array}{l}-0.00115 \\
(0.00154)\end{array}$ & $\begin{array}{c}0.0116^{*} \\
(0.00619)\end{array}$ \\
\hline 1or2_month & $\begin{array}{l}-0.00708^{*} \\
(0.00399)\end{array}$ & $\begin{array}{c}-0.00779^{* *} \\
(0.00344)\end{array}$ & $\begin{array}{c}-0.00476^{* * *} \\
(0.00113)\end{array}$ & $\begin{array}{c}0.00342 \\
(0.00415)\end{array}$ & $\begin{array}{r}-0.000161 \\
(0.00183)\end{array}$ & $\begin{array}{l}0.0173^{*} \\
(0.0101)\end{array}$ \\
\hline$<1$ _month & $\begin{array}{l}-0.00297 \\
(0.00494)\end{array}$ & $\begin{array}{l}-0.00559 \\
(0.00601)\end{array}$ & $\begin{array}{c}-0.00548^{* * *} \\
(0.00153)\end{array}$ & $\begin{array}{c}0.00483 \\
(0.00669)\end{array}$ & $\begin{array}{r}-0.000739 \\
(0.00268)\end{array}$ & $\begin{array}{c}0.0208 \\
(0.0130)\end{array}$ \\
\hline 0_in_3months & $\begin{array}{c}-0.00564^{*} \\
(0.00256)\end{array}$ & $\begin{array}{c}-0.00683^{* *} \\
(0.00335)\end{array}$ & $\begin{array}{l}-0.00529^{\star * *} \\
(0.000933)\end{array}$ & $\begin{array}{c}0.00838^{\star * \star} \\
(0.00292)\end{array}$ & $\begin{array}{l}-0.000545 \\
(0.00161)\end{array}$ & $\begin{array}{c}0.00451 \\
(0.00802)\end{array}$ \\
\hline 1_week & 0.00165 & -0.000254 & -0.000223 & 0.00341 & $-3.34 \mathrm{e}-05$ & $-0.0173^{* *}$ \\
\hline
\end{tabular}




\begin{tabular}{|c|c|c|c|c|c|c|c|}
\hline & $(0.00253)$ & $(0.00275)$ & $(0.000473)$ & $(0.00317)$ & $(0.000970)$ & $(0.00709)$ & \\
\hline 1or3_month & 0.00210 & 0.000275 & $-9.95 e-05$ & 0.000482 & 0.000898 & $-0.0422^{\star \star \star}$ & \\
\hline & $(0.00278)$ & $(0.00219)$ & $(0.000599)$ & $(0.00234)$ & $(0.00158)$ & $(0.00681)$ & \\
\hline Hardlyever_never & $0.0225^{\star * \star}$ & $0.0128^{\star \star \star}$ & $0.00605^{* * *}$ & $0.0191^{* * *}$ & $0.0106^{\star \star \star}$ & $-0.0920^{\star * \star}$ & \\
\hline & $(0.00190)$ & $(0.00289)$ & $(0.000569)$ & $(0.00314)$ & $(0.00134)$ & $(0.00704)$ & \\
\hline Smoking & $\begin{array}{l}-0.00424^{*} \\
(0.00226)\end{array}$ & $\begin{array}{l}0.0199^{\star * *} \\
(0.00298)\end{array}$ & $\begin{array}{c}-0.00185^{\star * *} \\
(0.000566)\end{array}$ & $\begin{array}{l}-4.97 e-05 \\
(0.00230)\end{array}$ & $\begin{array}{l}0.000481 \\
(0.00111)\end{array}$ & $\begin{array}{c}0.00185 \\
(0.00342)\end{array}$ & \\
\hline Overweight_Obese & $-0.00430^{* * *}$ & -0.000766 & $-0.00140^{* *}$ & $0.00506^{* *}$ & -0.000873 & $-0.0874^{* * *}$ & \\
\hline & $(0.00146)$ & $(0.00257)$ & $(0.000562)$ & $(0.00207)$ & $(0.000764)$ & $(0.00280)$ & \\
\hline Year Dummies & YES & YES & YES & YES & YES & YES & \\
\hline Country Dummies & YES & YES & YES & YES & YES & YES & \\
\hline Observations & 111,321 & 111,321 & 111,321 & 111,321 & 111,321 & 111,321 & 111,321 \\
\hline
\end{tabular}

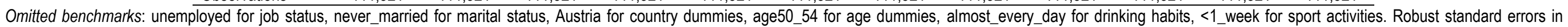
parentheses clustered at country level. ${ }^{* * *} p<0.01,{ }^{* *} p<0.05,{ }^{*} p<0.1$.

Table A5c - Education years and health functionalities (IV estimates)

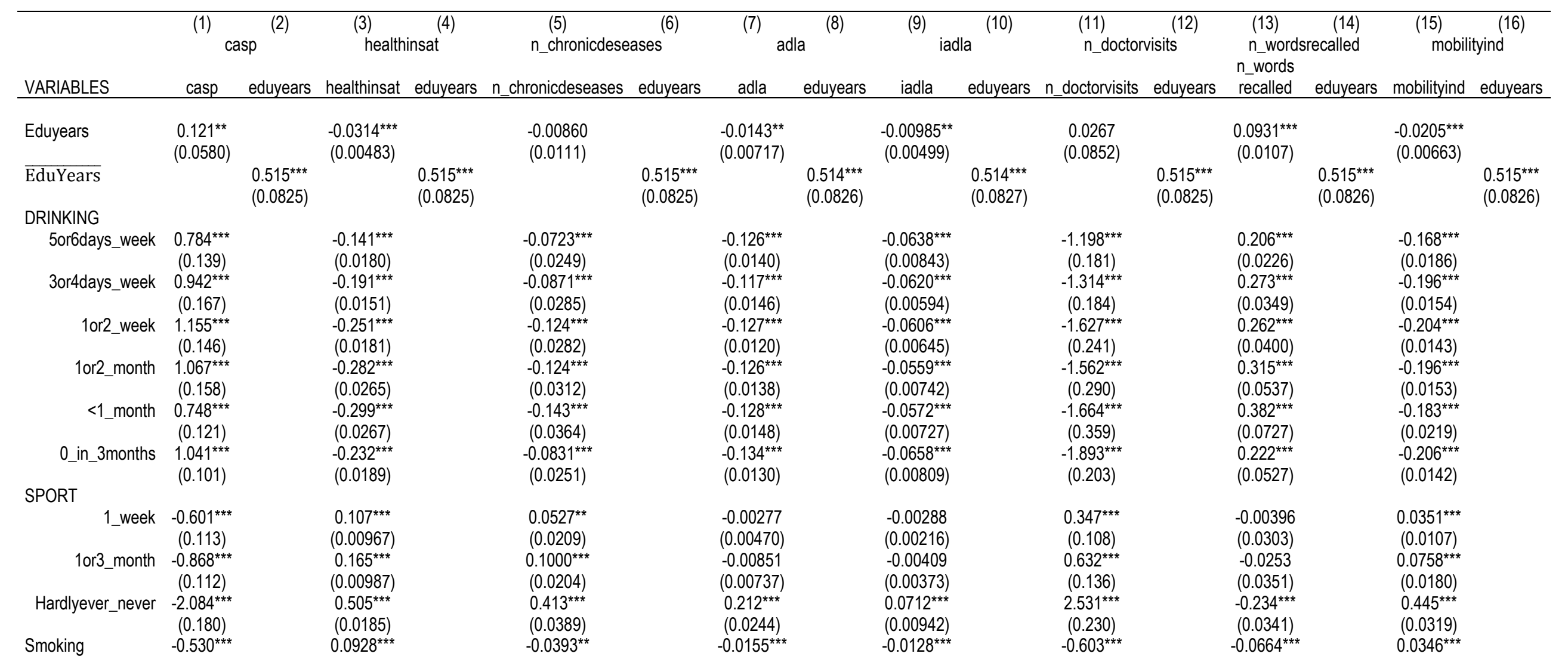




\begin{tabular}{|c|c|c|c|c|c|c|c|c|c|c|c|c|c|c|c|c|}
\hline \multirow{2}{*}{ Overweight_Obese } & $(0.0844)$ & & $(0.0175)$ & & $(0.0174)$ & & $(0.00504)$ & & $(0.00240)$ & & $(0.133)$ & & $(0.0209)$ & & $(0.00858)$ & \\
\hline & $-0.262^{* * *}$ & & $0.163^{* * *}$ & & $0.360^{* \star \star}$ & & $0.0195^{\star *}$ & & $-0.0200^{* * *}$ & & $0.692^{* * *}$ & & -0.0231 & & $0.131^{* * *}$ & \\
\hline & $(0.0687)$ & & $(0.0131)$ & & $(0.0113)$ & & $(0.00872)$ & & $(0.00491)$ & & $(0.0956)$ & & $(0.0183)$ & & $(0.0134)$ & \\
\hline Female & $\begin{array}{l}-0.0528 \\
(0.0943)\end{array}$ & & $\begin{array}{l}-0.0167 \\
(0.0222)\end{array}$ & & $\begin{array}{l}0.0376^{*} \\
(0.0193)\end{array}$ & & $\begin{array}{l}-0.0148 \\
(0.0111)\end{array}$ & & $\begin{array}{l}-0.0211^{* * *} \\
(0.00579)\end{array}$ & & $\begin{array}{c}0.170 \\
(0.114)\end{array}$ & & $\begin{array}{l}0.424^{* * *} \\
(0.0379)\end{array}$ & & $\begin{array}{l}0.0816^{* * *} \\
(0.0179)\end{array}$ & \\
\hline Age55_59 & $\begin{array}{c}0.0931 \\
(0.0997)\end{array}$ & & $\begin{array}{c}0.0969^{* * *} \\
(0.0192)\end{array}$ & & $\begin{array}{l}0.196^{* * *} \\
(0.0218)\end{array}$ & & $\begin{array}{l}-0.00322 \\
(0.00649)\end{array}$ & & $\begin{array}{l}-0.00427^{*} \\
(0.00240)\end{array}$ & & $\begin{array}{c}0.136 \\
(0.130)\end{array}$ & & $\begin{array}{c}-0.0938^{* * *} \\
(0.0256)\end{array}$ & & $\begin{array}{l}0.0334^{* * *} \\
(0.00873)\end{array}$ & \\
\hline Age60_64 & $\begin{array}{c}0.368^{* * *} \\
(0.142)\end{array}$ & & $\begin{array}{l}0.0835^{\star * *} \\
(0.0277)\end{array}$ & & $\begin{array}{l}0.332^{\star * *} \\
(0.0217)\end{array}$ & & $\begin{array}{c}0.00904 \\
(0.00965)\end{array}$ & & $\begin{array}{l}-0.000454 \\
(0.00431)\end{array}$ & & $\begin{array}{l}0.0579 \\
(0.156)\end{array}$ & & $\begin{array}{l}-0.190^{\star * *} \\
(0.0296)\end{array}$ & & $\begin{array}{l}0.0535^{\star * *} \\
(0.0162)\end{array}$ & \\
\hline Age65_69 & $\begin{array}{c}0.133 \\
(0.211)\end{array}$ & & $\begin{array}{l}0.133^{* * *} \\
(0.0378)\end{array}$ & & $\begin{array}{l}0.489^{\star * *} \\
(0.0357)\end{array}$ & & $\begin{array}{l}0.0325^{\star *} \\
(0.0151)\end{array}$ & & $\begin{array}{c}0.0110^{*} \\
(0.00560)\end{array}$ & & $\begin{array}{l}0.493^{*} \\
(0.253)\end{array}$ & & $\begin{array}{l}-0.378^{\star * *} \\
(0.0350)\end{array}$ & & $\begin{array}{l}0.123^{\star * \star} \\
(0.0288)\end{array}$ & \\
\hline Age70_74 & $\begin{array}{l}-0.235 \\
(0.230)\end{array}$ & & $\begin{array}{l}0.233^{* * *} \\
(0.0407)\end{array}$ & & $\begin{array}{l}0.656^{* * *} \\
(0.0340)\end{array}$ & & $\begin{array}{c}0.0604^{* * *} \\
(0.0157)\end{array}$ & & $\begin{array}{l}0.0200^{\star * *} \\
(0.00583)\end{array}$ & & $\begin{array}{c}0.853^{* * *} \\
(0.245)\end{array}$ & & $\begin{array}{l}-0.694^{* * *} \\
(0.0391)\end{array}$ & & $\begin{array}{l}0.215^{\star \star *} \\
(0.0368)\end{array}$ & \\
\hline Age75_79 & $\begin{array}{c}-0.744^{\star * *} \\
(0.274)\end{array}$ & & $\begin{array}{l}0.325^{\star \star \star} \\
(0.0456)\end{array}$ & & $\begin{array}{l}0.831^{* * *} \\
(0.0390)\end{array}$ & & $\begin{array}{l}0.129^{* * *} \\
(0.0257)\end{array}$ & & $\begin{array}{c}0.0577^{\star \star \star} \\
(0.0111)\end{array}$ & & $\begin{array}{c}1.384^{* * *} \\
(0.319)\end{array}$ & & $\begin{array}{c}-1.010^{\star * *} \\
(0.0435)\end{array}$ & & $\begin{array}{l}0.370^{\star * \star} \\
(0.0474)\end{array}$ & \\
\hline AgeAbove_80 & $\begin{array}{c}-1.790^{* * *} \\
(0.336)\end{array}$ & & $\begin{array}{l}0.429^{* * *} \\
(0.0447)\end{array}$ & & $\begin{array}{l}0.873^{* * *} \\
(0.0463)\end{array}$ & & $\begin{array}{l}0.401^{* * *} \\
(0.0457)\end{array}$ & & $\begin{array}{l}0.228^{* * *} \\
(0.0256)\end{array}$ & & $\begin{array}{l}1.294^{* * *} \\
(0.354)\end{array}$ & & $\begin{array}{l}-1.608^{* * *} \\
(0.0550)\end{array}$ & & $\begin{array}{l}0.706^{* * *} \\
(0.0528)\end{array}$ & \\
\hline Married & $\begin{array}{c}0.670^{* * *} \\
(0.125)\end{array}$ & & $\begin{array}{l}0.00134 \\
(0.0277)\end{array}$ & & $\begin{array}{c}0.0354 \\
(0.0302)\end{array}$ & & $\begin{array}{l}-0.00855 \\
(0.0110)\end{array}$ & & $\begin{array}{l}-0.0206^{* * *} \\
(0.00639)\end{array}$ & & $\begin{array}{l}0.0365 \\
(0.180)\end{array}$ & & $\begin{array}{l}0.177^{* * *} \\
(0.0303)\end{array}$ & & $\begin{array}{c}-0.0531^{* * *} \\
(0.0198)\end{array}$ & \\
\hline Reg_Partnership & $\begin{array}{l}0.617^{\star *} \\
(0.268)\end{array}$ & & $\begin{array}{l}-0.0143 \\
(0.0440)\end{array}$ & & $\begin{array}{c}0.0189 \\
(0.0658)\end{array}$ & & $\begin{array}{c}0.0123 \\
(0.0185)\end{array}$ & & $\begin{array}{l}-0.0241^{* * *} \\
(0.00725)\end{array}$ & & $\begin{array}{l}0.0986 \\
(0.325)\end{array}$ & & $\begin{array}{l}0.164^{* * *} \\
(0.0597)\end{array}$ & & $\begin{array}{l}-0.0212 \\
(0.0244)\end{array}$ & \\
\hline Separated & $\begin{array}{l}-0.408^{*} \\
(0.227)\end{array}$ & & $\begin{array}{c}0.0239 \\
(0.0262)\end{array}$ & & $\begin{array}{l}0.122^{* *} \\
(0.0495)\end{array}$ & & $\begin{array}{l}0.0508^{* *} \\
(0.0234)\end{array}$ & & $\begin{array}{c}-0.0169^{* * *} \\
(0.00596)\end{array}$ & & $\begin{array}{c}-0.00879 \\
(0.238)\end{array}$ & & $\begin{array}{c}0.138^{* *} \\
(0.0595)\end{array}$ & & $\begin{array}{l}-0.0433 \\
(0.0378)\end{array}$ & \\
\hline Divorced & $\begin{array}{l}-0.360^{*} \\
(0.198)\end{array}$ & & $\begin{array}{c}0.0309 \\
(0.0321)\end{array}$ & & $\begin{array}{l}0.124^{\star * *} \\
(0.0304)\end{array}$ & & $\begin{array}{l}0.0205^{\star} \\
(0.0121)\end{array}$ & & $\begin{array}{l}-0.0214^{* * *} \\
(0.00610)\end{array}$ & & $\begin{array}{c}0.424^{* * *} \\
(0.152)\end{array}$ & & $\begin{array}{l}0.148^{\star \star \star} \\
(0.0346)\end{array}$ & & $\begin{array}{l}0.00368 \\
(0.0198)\end{array}$ & \\
\hline Widowed & $\begin{array}{l}0.128 \\
(0.158)\end{array}$ & & $\begin{array}{l}0.00581 \\
(0.0280)\end{array}$ & & $\begin{array}{l}0.125^{\star \star *} \\
(0.0349)\end{array}$ & & $\begin{array}{l}0.0326^{* *} \\
(0.0135)\end{array}$ & & $\begin{array}{c}0.00438 \\
(0.00767)\end{array}$ & & $\begin{array}{l}0.105 \\
(0.196)\end{array}$ & & $\begin{array}{l}0.00363 \\
(0.0335)\end{array}$ & & $\begin{array}{l}0.0317^{*} \\
(0.0173)\end{array}$ & \\
\hline Retired & $\begin{array}{c}2.563^{* * *} \\
(0.236)\end{array}$ & & $\begin{array}{l}-0.428^{* * *} \\
(0.0428)\end{array}$ & & $\begin{array}{l}-0.279^{* * *} \\
(0.0399)\end{array}$ & & $\begin{array}{l}-0.234^{* * *} \\
(0.0425)\end{array}$ & & $\begin{array}{c}-0.0950^{* * *} \\
(0.0239)\end{array}$ & & $\begin{array}{c}-3.119^{* * *} \\
(0.403)\end{array}$ & & $\begin{array}{l}0.188^{* * *} \\
(0.0307)\end{array}$ & & $\begin{array}{l}-0.341^{* * *} \\
(0.0443)\end{array}$ & \\
\hline Employed & $\begin{array}{c}2.936^{\star \star *} \\
(0.257)\end{array}$ & & $\begin{array}{l}-0.649^{\star \star *} \\
(0.0395)\end{array}$ & & $\begin{array}{l}-0.509^{\star \star \star} \\
(0.0347)\end{array}$ & & $\begin{array}{l}-0.237^{\star \star *} \\
(0.0319)\end{array}$ & & $\begin{array}{c}-0.0771^{* \star *} \\
(0.0178)\end{array}$ & & $\begin{array}{c}-4.273^{\star \star \star} \\
(0.374)\end{array}$ & & $\begin{array}{l}0.263^{\star \star \star} \\
(0.0430)\end{array}$ & & $\begin{array}{l}-0.380^{* \star *} \\
(0.0335)\end{array}$ & \\
\hline Homemaker & $\begin{array}{c}2.295^{\star * *} \\
(0.231)\end{array}$ & & $\begin{array}{l}-0.476^{\star * *} \\
(0.0492)\end{array}$ & & $\begin{array}{l}-0.361^{* * *} \\
(0.0486)\end{array}$ & & $\begin{array}{l}-0.268^{\star * *} \\
(0.0426)\end{array}$ & & $\begin{array}{c}-0.0926^{* \star *} \\
(0.0268)\end{array}$ & & $\begin{array}{c}-3.311^{\star * \star} \\
(0.396)\end{array}$ & & $\begin{array}{c}0.109^{\star \star} \\
(0.0488)\end{array}$ & & $\begin{array}{l}-0.337^{\star \star *} \\
(0.0426)\end{array}$ & \\
\hline Other_Job & $\begin{array}{c}2.018^{* * *} \\
(0.284)\end{array}$ & & $\begin{array}{l}-0.460^{* * *} \\
(0.0457)\end{array}$ & & $\begin{array}{l}-0.339^{* * *} \\
(0.0496)\end{array}$ & & $\begin{array}{l}-0.161^{* * *} \\
(0.0400)\end{array}$ & & $\begin{array}{l}-0.0371 \\
(0.0244)\end{array}$ & & $\begin{array}{c}-3.085^{\star * *} \\
(0.430)\end{array}$ & & $\begin{array}{l}0.107^{\star *} \\
(0.0529)\end{array}$ & & $\begin{array}{l}-0.272^{* * *} \\
(0.0409)\end{array}$ & \\
\hline N_Children & $\begin{array}{c}-0.0748^{* *} \\
(0.0352)\end{array}$ & & $\begin{array}{l}-0.0115^{*} \\
(0.00681)\end{array}$ & & $\begin{array}{l}-0.0101^{*} \\
(0.00604)\end{array}$ & & $\begin{array}{l}-0.000868 \\
(0.00288)\end{array}$ & & $\begin{array}{c}0.00155 \\
(0.00164)\end{array}$ & & $\begin{array}{l}-0.00636 \\
(0.0472)\end{array}$ & & $\begin{array}{l}-0.0161^{*} \\
(0.00968)\end{array}$ & & $\begin{array}{c}0.00695 \\
(0.00429)\end{array}$ & \\
\hline N_Grandchildren & $\begin{array}{l}0.0300^{* *} \\
(0.0119)\end{array}$ & & $\begin{array}{l}0.00367^{*} \\
(0.00215)\end{array}$ & & $\begin{array}{l}0.0143^{* * *} \\
(0.00279)\end{array}$ & & $\begin{array}{l}0.00341^{*} \\
(0.00187)\end{array}$ & & $\begin{array}{l}0.00227^{* *} \\
(0.00115)\end{array}$ & & $\begin{array}{l}0.0460^{*} \\
(0.0268)\end{array}$ & & $\begin{array}{l}-0.00244 \\
(0.00410)\end{array}$ & & $\begin{array}{c}0.00580^{* * *} \\
(0.00210)\end{array}$ & \\
\hline Logincome & $\begin{array}{l}0.323^{* * *} \\
(0.0482)\end{array}$ & & $\begin{array}{l}-0.0242^{* * *} \\
(0.00699)\end{array}$ & & $\begin{array}{c}0.00995 \\
(0.00756)\end{array}$ & & $\begin{array}{c}0.00730^{* * *} \\
(0.00189)\end{array}$ & & $\begin{array}{c}0.00235 \\
(0.00190)\end{array}$ & & $\begin{array}{l}0.0730^{*} \\
(0.0404)\end{array}$ & & $\begin{array}{c}0.0559^{* * *} \\
(0.0153)\end{array}$ & & $\begin{array}{l}-0.000855 \\
(0.00425)\end{array}$ & \\
\hline $\begin{array}{l}\text { Year dummies } \\
\text { Country dummies }\end{array}$ & $\begin{array}{l}\text { YES } \\
\text { YES }\end{array}$ & & $\begin{array}{l}\text { YES } \\
\text { YES }\end{array}$ & & $\begin{array}{l}\text { YES } \\
\text { YES }\end{array}$ & & $\begin{array}{l}\text { YES } \\
\text { YES }\end{array}$ & & $\begin{array}{l}\text { YES } \\
\text { YES }\end{array}$ & & $\begin{array}{l}\text { YES } \\
\text { YES }\end{array}$ & & $\begin{array}{l}\text { YES } \\
\text { YES }\end{array}$ & & $\begin{array}{l}\text { YES } \\
\text { YES }\end{array}$ & \\
\hline Observations & 107,822 & 107,822 & 108,259 & 108,259 & 108,239 & 108,239 & 108,249 & 108,249 & 108,249 & 108,249 & 108,155 & 108,155 & 108,169 & 108,169 & 108,249 & 108,249 \\
\hline
\end{tabular}

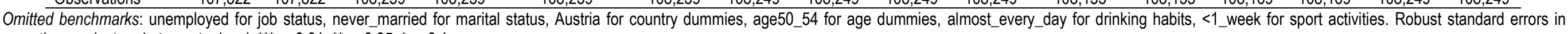
parentheses clustered at country level. ${ }^{* \star *} p<0.01,{ }^{* *} p<0.05,{ }^{*} p<0.1$. 
Table A5d - Education years and specific illnesses (IV estimates)

\begin{tabular}{|c|c|c|c|c|c|c|c|c|c|c|c|c|c|c|}
\hline \multirow[b]{3}{*}{ VARIABLES } & \multirow{2}{*}{\multicolumn{2}{|c|}{$\begin{array}{l}\text { (1) } \\
\text { longterm } \\
\text { illness }\end{array}$}} & (3) & (4) & (5) & (6) & (7) & (8) & (9) & (10) & (11) & (12) & (13) & (14) \\
\hline & & & \multicolumn{2}{|c|}{ heartattack } & \multicolumn{2}{|c|}{ hypertension } & \multicolumn{2}{|c|}{ stroke } & \multicolumn{2}{|c|}{ diabetes } & \multicolumn{2}{|c|}{ asthma } & \multicolumn{2}{|c|}{ arthritis } \\
\hline & 2nd stage & 1st stage & 2nd stage & 1 st stage & 2nd stage & 1st stage & 2nd stage & 1st stage & 2nd stage & 1st stage & 2nd stage & 1 st stage & 2nd stage & 1st stage \\
\hline Eduyears & $\begin{array}{l}-0.000535 \\
(0.00221)\end{array}$ & & $\begin{array}{l}-0.000815 \\
(0.000816)\end{array}$ & & $\begin{array}{c}-0.00452^{* * *} \\
(0.00167)\end{array}$ & & $\begin{array}{l}-0.000500 \\
(0.000705)\end{array}$ & & $\begin{array}{c}-0.000826 \\
(0.00286)\end{array}$ & & $\begin{array}{l}-0.000851 \\
(0.000558)\end{array}$ & & $\begin{array}{l}-0.00188 \\
(0.00216)\end{array}$ & \\
\hline$\overline{\text { EduYears }}$ & & $\begin{array}{l}0.515^{\star * *} \\
(0.0825)\end{array}$ & & $\begin{array}{l}0.515^{\star * *} \\
(0.0825)\end{array}$ & & $\begin{array}{l}0.515^{\star \star *} \\
(0.0825)\end{array}$ & & $\begin{array}{l}0.515^{\star * *} \\
(0.0825)\end{array}$ & & $\begin{array}{l}0.515^{\star * *} \\
(0.0825)\end{array}$ & & $\begin{array}{l}0.515^{\star \star *} \\
(0.0825)\end{array}$ & & $\begin{array}{l}0.515^{\star \star *} \\
(0.0825)\end{array}$ \\
\hline DRINKING & & & & & & & & & & & & & & \\
\hline 5or6days_week & $\begin{array}{l}-0.0245^{\star * *} \\
(0.00715)\end{array}$ & & $\begin{array}{c}-0.0272^{* * *} \\
(0.00733)\end{array}$ & & $\begin{array}{l}-0.00234 \\
(0.00732)\end{array}$ & & $\begin{array}{l}-0.0183^{* * *} \\
(0.00478)\end{array}$ & & $\begin{array}{l}-0.0254^{* * *} \\
(0.00301)\end{array}$ & & $\begin{array}{c}-0.00395^{\star *} \\
(0.00164)\end{array}$ & & $\begin{array}{l}0.000453 \\
(0.00605)\end{array}$ & \\
\hline 3or4days_week & $\begin{array}{l}-0.0473^{* * *} \\
(0.00826)\end{array}$ & & $\begin{array}{l}-0.0301^{* * *} \\
(0.00716)\end{array}$ & & $\begin{array}{l}-0.00985 \\
(0.00733)\end{array}$ & & $\begin{array}{l}-0.0198^{* * *} \\
(0.00427)\end{array}$ & & $\begin{array}{l}-0.0362^{* * *} \\
(0.00373)\end{array}$ & & $\begin{array}{l}-0.00453^{* *} \\
(0.00221)\end{array}$ & & $\begin{array}{c}0.00545 \\
(0.00582)\end{array}$ & \\
\hline 1or2_week & $\begin{array}{l}-0.0679^{* * *} \\
(0.00667)\end{array}$ & & $\begin{array}{l}-0.0348^{* * *} \\
(0.00617)\end{array}$ & & $\begin{array}{l}-0.0180^{* * *} \\
(0.00638)\end{array}$ & & $\begin{array}{l}-0.0216^{* * *} \\
(0.00457)\end{array}$ & & $\begin{array}{l}-0.0457^{* * *} \\
(0.00477)\end{array}$ & & $\begin{array}{c}-0.00435^{\star \star *} \\
(0.00161)\end{array}$ & & $\begin{array}{c}0.00156 \\
(0.00624)\end{array}$ & \\
\hline 1or2_month & $\begin{array}{c}-0.0588^{\star \star *} \\
(0.0102)\end{array}$ & & $\begin{array}{c}-0.0341^{* * *} \\
(0.00787)\end{array}$ & & $\begin{array}{c}-0.0161^{\star * \star} \\
(0.00540)\end{array}$ & & $\begin{array}{c}-0.0246^{* * *} \\
(0.00434)\end{array}$ & & $\begin{array}{c}-0.0496^{* * *} \\
(0.00559)\end{array}$ & & $\begin{array}{c}-0.00514^{* *} \\
(0.00219)\end{array}$ & & $\begin{array}{c}0.00155 \\
(0.00794)\end{array}$ & \\
\hline$<1$ _month & $\begin{array}{c}-0.0773^{* * *} \\
(0.0113)\end{array}$ & & $\begin{array}{c}-0.0392^{* * *} \\
(0.00663)\end{array}$ & & $\begin{array}{l}-0.0203^{* *} \\
(0.00793)\end{array}$ & & $\begin{array}{l}-0.0234^{* * *} \\
(0.00369)\end{array}$ & & $\begin{array}{l}-0.0626^{* * *} \\
(0.00568)\end{array}$ & & $\begin{array}{l}-0.00601^{* *} \\
(0.00247)\end{array}$ & & $\begin{array}{l}-0.00261 \\
(0.00832)\end{array}$ & \\
\hline 0_in_3months & $\begin{array}{l}-0.0623^{* * *} \\
(0.00741)\end{array}$ & & $\begin{array}{l}-0.0353^{* * *} \\
(0.00562)\end{array}$ & & $\begin{array}{l}-0.00215 \\
(0.00684)\end{array}$ & & $\begin{array}{l}-0.0193^{* * *} \\
(0.00349)\end{array}$ & & $\begin{array}{l}-0.0544^{* * *} \\
(0.00651)\end{array}$ & & $\begin{array}{l}-0.00158^{\star *} \\
(0.000768)\end{array}$ & & $\begin{array}{c}0.00531 \\
(0.00726)\end{array}$ & \\
\hline SPORT & & & & & & & & & & & & & & \\
\hline 1_week & $\begin{array}{c}0.00938 \\
(0.00642)\end{array}$ & & $\begin{array}{c}0.00433 \\
(0.00335)\end{array}$ & & $\begin{array}{l}0.0190^{* * *} \\
(0.00645)\end{array}$ & & $\begin{array}{l}0.000132 \\
(0.00123)\end{array}$ & & $\begin{array}{l}0.00634^{*} \\
(0.00333)\end{array}$ & & $\begin{array}{l}0.000478 \\
(0.00160)\end{array}$ & & $\begin{array}{c}0.00525 \\
(0.00409)\end{array}$ & \\
\hline 1or3_month & $\begin{array}{l}0.0462^{* * *} \\
(0.00790)\end{array}$ & & $\begin{array}{l}0.0200^{* * *} \\
(0.00448)\end{array}$ & & $\begin{array}{l}0.0271^{* * *} \\
(0.00807)\end{array}$ & & $\begin{array}{c}0.00233 \\
(0.00200)\end{array}$ & & $\begin{array}{l}0.0136^{* * *} \\
(0.00392)\end{array}$ & & $\begin{array}{c}0.00285 \\
(0.00184)\end{array}$ & & $\begin{array}{c}0.00750 \\
(0.00564)\end{array}$ & \\
\hline Hardlyever_never & $\begin{array}{c}0.160^{* * *} \\
(0.00988)\end{array}$ & & $\begin{array}{l}0.0675^{\star * \star} \\
(0.00642)\end{array}$ & & $\begin{array}{l}0.0656^{* * *} \\
(0.00803)\end{array}$ & & $\begin{array}{l}0.0301^{* * *} \\
(0.00515)\end{array}$ & & $\begin{array}{l}0.0457^{* \star \star} \\
(0.00450)\end{array}$ & & $\begin{array}{l}0.0122^{* * *} \\
(0.00214)\end{array}$ & & $\begin{array}{l}0.0622^{* * *} \\
(0.00714)\end{array}$ & \\
\hline Smoking & $\begin{array}{l}-0.00928^{\star} \\
(0.00545)\end{array}$ & & $\begin{array}{l}-0.0174^{* * *} \\
(0.00268)\end{array}$ & & $\begin{array}{l}-0.0393^{* * *} \\
(0.00538)\end{array}$ & & $\begin{array}{r}-0.000337 \\
(0.00179)\end{array}$ & & $\begin{array}{l}-0.00712^{* *} \\
(0.00303)\end{array}$ & & $\begin{array}{l}-0.00283 \\
(0.00193)\end{array}$ & & $\begin{array}{l}-0.00306 \\
(0.00407)\end{array}$ & \\
\hline Overweight_Obese & $\begin{array}{l}0.0713^{\star \star *} \\
(0.00488)\end{array}$ & & $\begin{array}{l}0.0215^{\star \star *} \\
(0.00404)\end{array}$ & & $\begin{array}{c}0.163^{* * *} \\
(0.00701)\end{array}$ & & $\begin{array}{l}0.000735 \\
(0.00119)\end{array}$ & & $\begin{array}{l}0.0701^{* * *} \\
(0.00344)\end{array}$ & & $\begin{array}{c}0.00476 * \star * \\
(0.00167)\end{array}$ & & $\begin{array}{l}0.0474^{\star * \star} \\
(0.00487)\end{array}$ & \\
\hline Female & $\begin{array}{c}0.00678 \\
(0.00749)\end{array}$ & & $\begin{array}{c}-0.0617^{* * *} \\
(0.00369)\end{array}$ & & $\begin{array}{l}0.0219^{* * *} \\
(0.00567)\end{array}$ & & $\begin{array}{l}-0.0168^{* * *} \\
(0.00276)\end{array}$ & & $\begin{array}{l}-0.0311^{* * *} \\
(0.00442)\end{array}$ & & $\begin{array}{c}0.00182 \\
(0.00150)\end{array}$ & & $\begin{array}{l}0.103^{* * *} \\
(0.0108)\end{array}$ & \\
\hline Age55_59 & $\begin{array}{l}0.0397^{\star * *} \\
(0.00865)\end{array}$ & & $\begin{array}{c}0.00721^{* * *} \\
(0.00214)\end{array}$ & & $\begin{array}{l}0.0623^{* * *} \\
(0.00757)\end{array}$ & & $\begin{array}{c}0.00266^{*} \\
(0.00140)\end{array}$ & & $\begin{array}{l}0.0151^{* * *} \\
(0.00335)\end{array}$ & & $\begin{array}{l}-0.00436^{*} \\
(0.00230)\end{array}$ & & $\begin{array}{l}0.0462^{* * *} \\
(0.00654)\end{array}$ & \\
\hline Age60_64 & $\begin{array}{l}0.0401^{* * *} \\
(0.00904)\end{array}$ & & $\begin{array}{l}0.0199^{\star \star *} \\
(0.00385)\end{array}$ & & $\begin{array}{c}0.112^{\star \star *} \\
(0.00953)\end{array}$ & & $\begin{array}{l}0.00371^{* *} \\
(0.00156)\end{array}$ & & $\begin{array}{l}0.0254^{\star * *} \\
(0.00402)\end{array}$ & & $\begin{array}{l}-0.00503^{*} \\
(0.00286)\end{array}$ & & $\begin{array}{l}0.0670^{\star * \star} \\
(0.00910)\end{array}$ & \\
\hline Age65_69 & $\begin{array}{c}0.0597^{* * *} \\
(0.0113)\end{array}$ & & $\begin{array}{l}0.0396^{* * *} \\
(0.00346)\end{array}$ & & $\begin{array}{l}0.156^{* * *} \\
(0.0137)\end{array}$ & & $\begin{array}{l}0.0103^{* * *} \\
(0.00239)\end{array}$ & & $\begin{array}{l}0.0471^{* * *} \\
(0.00744)\end{array}$ & & $\begin{array}{c}-0.00754^{* *} \\
(0.00351)\end{array}$ & & $\begin{array}{c}0.0876^{* * *} \\
(0.0137)\end{array}$ & \\
\hline Age70_74 & $\begin{array}{c}0.0847^{* * *} \\
(0.0129)\end{array}$ & & $\begin{array}{l}0.0773^{* * *} \\
(0.00453)\end{array}$ & & $\begin{array}{l}0.191^{\star \star \star} \\
(0.0120)\end{array}$ & & $\begin{array}{l}0.0173^{* * *} \\
(0.00332)\end{array}$ & & $\begin{array}{l}0.0520^{* * *} \\
(0.00547)\end{array}$ & & $\begin{array}{l}-0.00404 \\
(0.00382)\end{array}$ & & $\begin{array}{c}0.1000^{* * *} \\
(0.0161)\end{array}$ & \\
\hline Age75_79 & $\begin{array}{l}0.107^{\star \star \star} \\
(0.0169)\end{array}$ & & $\begin{array}{c}0.120^{* * *} \\
(0.00647)\end{array}$ & & $\begin{array}{l}0.199^{* * *} \\
(0.0119)\end{array}$ & & $\begin{array}{l}0.0355^{\star * *} \\
(0.00441)\end{array}$ & & $\begin{array}{l}0.0552^{\star * *} \\
(0.00827)\end{array}$ & & $\begin{array}{l}-0.00533 \\
(0.00361)\end{array}$ & & $\begin{array}{l}0.123^{* * *} \\
(0.0168)\end{array}$ & \\
\hline AgeAbove_80 & $\begin{array}{l}0.124^{\star * \star} \\
(0.0168)\end{array}$ & & $\begin{array}{l}0.143^{* * *} \\
(0.0106)\end{array}$ & & $\begin{array}{l}0.173^{\star \star *} \\
(0.0147)\end{array}$ & & $\begin{array}{l}0.0459^{* * *} \\
(0.00524)\end{array}$ & & $\begin{array}{l}0.0415^{\star * *} \\
(0.00794)\end{array}$ & & $\begin{array}{l}-0.00823^{*} \\
(0.00433)\end{array}$ & & $\begin{array}{l}0.146^{* * *} \\
(0.0183)\end{array}$ & \\
\hline Married & $\begin{array}{c}-0.00692 \\
(0.00785)\end{array}$ & & $\begin{array}{c}0.0122^{*} \\
(0.00739)\end{array}$ & & $\begin{array}{c}0.0140 \\
(0.00958)\end{array}$ & & $\begin{array}{c}0.00400 \\
(0.00318)\end{array}$ & & $\begin{array}{l}-0.00819 \\
(0.00597)\end{array}$ & & $\begin{array}{l}-0.00204 \\
(0.00314)\end{array}$ & & $\begin{array}{c}-0.00290 \\
(0.00868)\end{array}$ & \\
\hline
\end{tabular}




\begin{tabular}{|c|c|c|c|c|c|c|c|c|c|c|c|c|c|c|}
\hline Reg_Partnership & $\begin{array}{l}-0.00833 \\
(0.0176)\end{array}$ & & $\begin{array}{l}0.00812 \\
(0.0164)\end{array}$ & & $\begin{array}{l}-0.0133 \\
(0.0159)\end{array}$ & & $\begin{array}{c}0.00223 \\
(0.00478)\end{array}$ & & $\begin{array}{l}-0.00805 \\
(0.0111)\end{array}$ & & $\begin{array}{c}0.00501 \\
(0.00749)\end{array}$ & & $\begin{array}{l}-0.00249 \\
(0.0134)\end{array}$ & \\
\hline Separated & $\begin{array}{l}0.00808 \\
(0.0189)\end{array}$ & & $\begin{array}{l}0.00843 \\
(0.0133)\end{array}$ & & $\begin{array}{l}0.00942 \\
(0.0154)\end{array}$ & & $\begin{array}{l}0.0240^{* \star *} \\
(0.00740)\end{array}$ & & $\begin{array}{c}0.0175 \\
(0.0131)\end{array}$ & & $\begin{array}{l}-0.00253 \\
(0.00437)\end{array}$ & & $\begin{array}{l}0.00936 \\
(0.0102)\end{array}$ & \\
\hline Divorced & $\begin{array}{l}0.0331^{* * *} \\
(0.0113)\end{array}$ & & $\begin{array}{l}0.0238^{* * *} \\
(0.00904)\end{array}$ & & $\begin{array}{l}-0.00210 \\
(0.00807)\end{array}$ & & $\begin{array}{l}0.00643^{*} \\
(0.00390)\end{array}$ & & $\begin{array}{l}-0.00412 \\
(0.00653)\end{array}$ & & $\begin{array}{c}0.00863^{* * *} \\
(0.00296)\end{array}$ & & $\begin{array}{l}0.0292^{* * *} \\
(0.00934)\end{array}$ & \\
\hline Widowed & $\begin{array}{c}0.00275 \\
(0.00973)\end{array}$ & & $\begin{array}{l}0.0170^{* * *} \\
(0.00639)\end{array}$ & & $\begin{array}{l}0.0458^{\star * *} \\
(0.0112)\end{array}$ & & $\begin{array}{c}0.00230 \\
(0.00371)\end{array}$ & & $\begin{array}{c}0.00523 \\
(0.00709)\end{array}$ & & $\begin{array}{l}0.000719 \\
(0.00354)\end{array}$ & & $\begin{array}{l}0.0196^{*} \\
(0.0103)\end{array}$ & \\
\hline Retired & $\begin{array}{l}-0.180^{* * *} \\
(0.0197)\end{array}$ & & $\begin{array}{l}-0.0368^{* * *} \\
(0.00758)\end{array}$ & & $\begin{array}{l}-0.0265^{\star * *} \\
(0.00896)\end{array}$ & & $\begin{array}{c}-0.0286^{\star * *} \\
(0.00492)\end{array}$ & & $\begin{array}{c}-0.0289^{* * *} \\
(0.00697)\end{array}$ & & $\begin{array}{l}-0.00927^{* *} \\
(0.00438)\end{array}$ & & $\begin{array}{c}-0.0726^{* * *} \\
(0.00622)\end{array}$ & \\
\hline Employed & $\begin{array}{l}-0.277^{* * *} \\
(0.0177)\end{array}$ & & $\begin{array}{l}-0.0678^{* * *} \\
(0.00719)\end{array}$ & & $\begin{array}{c}-0.0552^{* * *} \\
(0.0112)\end{array}$ & & $\begin{array}{l}-0.0425^{\star \star *} \\
(0.00448)\end{array}$ & & $\begin{array}{c}-0.0489^{* * *} \\
(0.00668)\end{array}$ & & $\begin{array}{l}-0.0160^{* * *} \\
(0.00469)\end{array}$ & & $\begin{array}{c}-0.0983^{* * *} \\
(0.00780)\end{array}$ & \\
\hline Homemaker & $\begin{array}{l}-0.219^{* * *} \\
(0.0203)\end{array}$ & & $\begin{array}{l}-0.0540^{* * *} \\
(0.00843)\end{array}$ & & $\begin{array}{c}-0.0268^{* *} \\
(0.0111)\end{array}$ & & $\begin{array}{c}-0.0359^{* * *} \\
(0.00627)\end{array}$ & & $\begin{array}{l}-0.0287^{\star * \star} \\
(0.00848)\end{array}$ & & $\begin{array}{l}-0.0116^{* * *} \\
(0.00427)\end{array}$ & & $\begin{array}{c}-0.0690^{* * *} \\
(0.0138)\end{array}$ & \\
\hline Other_Job & $\begin{array}{l}-0.178^{* * *} \\
(0.0302)\end{array}$ & & $\begin{array}{c}-0.0380^{* * *} \\
(0.0108)\end{array}$ & & $\begin{array}{l}-0.0405^{\star *} \\
(0.0182)\end{array}$ & & $\begin{array}{c}-0.0258^{* * *} \\
(0.00712)\end{array}$ & & $\begin{array}{c}-0.0343^{* *} \\
(0.0159)\end{array}$ & & $\begin{array}{l}-0.0226^{* * *} \\
(0.00741)\end{array}$ & & $\begin{array}{c}-0.0617^{\star * *} \\
(0.0124)\end{array}$ & \\
\hline N_Children & $\begin{array}{l}-0.00268 \\
(0.00220)\end{array}$ & & $\begin{array}{l}0.000288 \\
(0.00137)\end{array}$ & & $\begin{array}{l}-0.00154 \\
(0.00135)\end{array}$ & & $\begin{array}{c}0.00122 \\
(0.000966)\end{array}$ & & $\begin{array}{c}0.00191 \\
(0.00139)\end{array}$ & & $\begin{array}{l}0.000855^{*} \\
(0.000513)\end{array}$ & & $\begin{array}{c}0.00129 \\
(0.00203)\end{array}$ & \\
\hline N_Grandchildren & $\begin{array}{l}0.00406^{* * *} \\
(0.000615)\end{array}$ & & $\begin{array}{l}0.00268^{* * *} \\
(0.000640)\end{array}$ & & $\begin{array}{l}0.00213^{* * *} \\
(0.000659)\end{array}$ & & $\begin{array}{c}0.000118 \\
(0.000448)\end{array}$ & & $\begin{array}{l}0.00147^{* *} \\
(0.000620)\end{array}$ & & $\begin{array}{l}0.000629^{* * *} \\
(0.000243)\end{array}$ & & $\begin{array}{l}0.00218^{* *} \\
(0.000907)\end{array}$ & \\
\hline Logincome & $\begin{array}{l}0.00561^{* *} \\
(0.00270)\end{array}$ & & $\begin{array}{c}0.00117 \\
(0.00132)\end{array}$ & & $\begin{array}{l}-0.00122 \\
(0.00185)\end{array}$ & & $\begin{array}{l}0.00147^{* *} \\
(0.000631)\end{array}$ & & $\begin{array}{c}-0.000862 \\
(0.00120)\end{array}$ & & $\begin{array}{l}0.00219^{* *} \\
(0.00107)\end{array}$ & & $\begin{array}{l}0.000187 \\
(0.00152)\end{array}$ & \\
\hline $\begin{array}{l}\text { Year dummies } \\
\text { Country dummies }\end{array}$ & $\begin{array}{l}\text { YES } \\
\text { YES }\end{array}$ & & $\begin{array}{l}\text { YES } \\
\text { YES }\end{array}$ & & $\begin{array}{l}\text { YES } \\
\text { YES }\end{array}$ & & $\begin{array}{l}\text { YES } \\
\text { YES }\end{array}$ & & $\begin{array}{l}\text { YES } \\
\text { YES }\end{array}$ & & $\begin{array}{l}\text { YES } \\
\text { YES }\end{array}$ & & $\begin{array}{l}\text { YES } \\
\text { YES }\end{array}$ & \\
\hline Observations & 111,321 & 111,321 & 111,321 & 111,321 & 111,321 & 111,321 & 111,321 & 111,321 & 111,321 & 111,321 & 111,321 & 111,321 & 111,321 & 111,321 \\
\hline
\end{tabular}

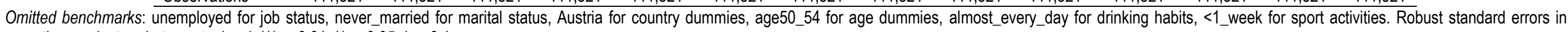
parentheses clustered at country level. ${ }^{* * *} p<0.01,{ }^{* *} p<0.05,{ }^{*} p<0.1$.

Table A5d - Education years and specific illnesses (IV estimates) - continued

\begin{tabular}{|c|c|c|c|c|c|c|c|c|c|c|c|c|}
\hline \multirow[b]{3}{*}{ VARIABLES } & (15) & (16) & (17) & (18) & (19) & (20) & (21) & (22) & (23) & (24) & (25) & (26) \\
\hline & \multicolumn{2}{|c|}{ cancer } & \multicolumn{2}{|c|}{ ulcer } & \multicolumn{2}{|c|}{ parkinson } & \multicolumn{2}{|c|}{ cataracts } & \multicolumn{2}{|c|}{ fracture } & \multicolumn{2}{|c|}{ none } \\
\hline & 2nd stage & 1st stage & 2nd stage & 1st stage & 2nd stage & 1st stage & 2nd stage & 1st stage & 2nd stage & 1st stage & 2nd stage & 1st stage \\
\hline Eduyears & $\begin{array}{l}0.00262^{* * *} \\
(0.000931)\end{array}$ & & $\begin{array}{l}-0.000775 \\
(0.000871)\end{array}$ & & $\begin{array}{c}-0.000459 \\
(0.000423)\end{array}$ & & $\begin{array}{l}0.00294^{*} \\
(0.00174)\end{array}$ & & $\begin{array}{l}-0.000368 \\
(0.000535)\end{array}$ & & $\begin{array}{l}0.000208 \\
(0.00185)\end{array}$ & \\
\hline$\overline{\text { EduYears }}$ & & $\begin{array}{l}0.515^{\star \star *} \\
(0.0825)\end{array}$ & & $\begin{array}{l}0.515^{\star \star \star} \\
(0.0825)\end{array}$ & & $\begin{array}{l}0.515^{\star * *} \\
(0.0825)\end{array}$ & & $\begin{array}{l}0.515^{\star \star \star} \\
(0.0825)\end{array}$ & & $\begin{array}{l}0.515^{\star * *} \\
(0.0825)\end{array}$ & & $\begin{array}{l}0.515^{* * *} \\
(0.0825)\end{array}$ \\
\hline DRINKING & & & & & & & & & & & & \\
\hline 5or6days_week & $\begin{array}{c}-0.00639^{\star * *} \\
(0.00202)\end{array}$ & & $\begin{array}{c}-0.00352 \\
(0.00412)\end{array}$ & & $\begin{array}{c}-0.00361^{* * *} \\
(0.000909)\end{array}$ & & $\begin{array}{c}0.00436 \\
(0.00445)\end{array}$ & & $\begin{array}{c}-0.00118 \\
(0.00173)\end{array}$ & & $\begin{array}{c}-0.00480 \\
(0.00717)\end{array}$ & \\
\hline 3or4days_week & $\begin{array}{c}-0.00483^{* *} \\
(0.00213)\end{array}$ & & $\begin{array}{l}-0.00320 \\
(0.00362)\end{array}$ & & $\begin{array}{c}-0.00377^{\star * *} \\
(0.00106)\end{array}$ & & $\begin{array}{c}0.00474 \\
(0.00383)\end{array}$ & & $\begin{array}{l}-0.00212 \\
(0.00139)\end{array}$ & & $\begin{array}{c}0.00671 \\
(0.00629)\end{array}$ & \\
\hline 1or2_week & $\begin{array}{c}-0.00700^{* * *} \\
(0.00227)\end{array}$ & & $\begin{array}{l}-0.00591 \\
(0.00454)\end{array}$ & & $\begin{array}{c}-0.00466^{* * *} \\
(0.00105)\end{array}$ & & $\begin{array}{c}0.00307 \\
(0.00409)\end{array}$ & & $\begin{array}{l}-0.00123 \\
(0.00156)\end{array}$ & & $\begin{array}{c}0.0119^{*} \\
(0.00632)\end{array}$ & \\
\hline 1or2_month & $-0.00726^{*}$ & & $-0.00784^{* *}$ & & $-0.00474^{* * *}$ & & 0.00311 & & -0.000180 & & $0.0174^{*}$ & \\
\hline
\end{tabular}




\begin{tabular}{|c|c|c|c|c|c|c|}
\hline \multirow{3}{*}{$<1$ _month } & $(0.00393)$ & $(0.00346)$ & $(0.00114)$ & $(0.00426)$ & $(0.00186)$ & (0.0102) \\
\hline & -0.00317 & -0.00564 & $-0.00545^{\star * *}$ & 0.00451 & -0.000755 & 0.0209 \\
\hline & $(0.00494)$ & $(0.00602)$ & $(0.00151)$ & $(0.00661)$ & $(0.00270)$ & $(0.0129)$ \\
\hline 0_in_3months & $\begin{array}{c}-0.00566^{* *} \\
(0.00253)\end{array}$ & $\begin{array}{c}-0.00684^{* *} \\
(0.00336)\end{array}$ & $\begin{array}{c}-0.00525^{\star * *} \\
(0.000945)\end{array}$ & $\begin{array}{c}0.00842^{* * *} \\
(0.00296)\end{array}$ & $\begin{array}{r}-0.000515 \\
(0.00162)\end{array}$ & $\begin{array}{c}0.00415 \\
(0.00802)\end{array}$ \\
\hline \multicolumn{7}{|l|}{ SPORT } \\
\hline \multirow[t]{2}{*}{ 1_week } & 0.00170 & -0.000248 & -0.000213 & 0.00353 & $-9.00 \mathrm{e}-06$ & $-0.0176^{\star *}$ \\
\hline & $(0.00253)$ & $(0.00274)$ & $(0.000465)$ & $(0.00319)$ & $(0.000980)$ & $(0.00716)$ \\
\hline \multirow[t]{2}{*}{ 1or3_month } & 0.00210 & 0.000262 & $-6.23 e-05$ & 0.000585 & 0.000938 & $-0.0426^{\star * *}$ \\
\hline & $(0.00279)$ & $(0.00220)$ & $(0.000578)$ & $(0.00237)$ & $(0.00159)$ & $(0.00686)$ \\
\hline \multirow[t]{2}{*}{ Hardlyever_never } & $0.0226^{\star * *}$ & $0.0128^{* * *}$ & $0.00631^{* \star *}$ & $0.0198^{* * *}$ & $0.0108^{* * *}$ & $-0.0934^{* * *}$ \\
\hline & $(0.00187)$ & $(0.00287)$ & $(0.000697)$ & $(0.00320)$ & $(0.00135)$ & $(0.00709)$ \\
\hline \multirow[t]{2}{*}{ Smoking } & $-0.00436^{*}$ & $0.0199^{\star * \star}$ & $-0.00207^{\star * \star}$ & -0.000820 & 0.000251 & 0.00335 \\
\hline & $(0.00226)$ & $(0.00303)$ & $(0.000587)$ & $(0.00232)$ & $(0.00110)$ & $(0.00334)$ \\
\hline \multirow[t]{2}{*}{ Overweight_Obese } & $-0.00435^{* * *}$ & -0.000754 & $-0.00150^{\star * *}$ & $0.00470^{\star *}$ & -0.000985 & $-0.0870^{* * *}$ \\
\hline & $(0.00145)$ & $(0.00256)$ & $(0.000556)$ & $(0.00208)$ & $(0.000779)$ & $(0.00279)$ \\
\hline \multirow[t]{2}{*}{ Female } & $0.00486^{\star *}$ & $-0.0104^{\star \star *}$ & $-0.00324^{\star * *}$ & $0.0215^{\star * *}$ & $0.00230^{*}$ & $-0.0159^{\star * *}$ \\
\hline & $(0.00206)$ & $(0.00262)$ & $(0.000602)$ & $(0.00241)$ & $(0.00136)$ & $(0.00468)$ \\
\hline \multirow[t]{2}{*}{ Age55_59 } & $0.00450^{\star}$ & -0.00149 & $-2.62 e-05$ & $0.00524^{* \star *}$ & 0.000144 & $-0.0835^{\star \star *}$ \\
\hline & $(0.00267)$ & $(0.00400)$ & $(0.000468)$ & $(0.00171)$ & $(0.00135)$ & $(0.0109)$ \\
\hline \multirow{2}{*}{ Age60_64 } & $0.00864^{\star * *}$ & -0.000490 & $0.00152^{*}$ & $0.0194^{* * *}$ & 0.00182 & $-0.125^{\star \star *}$ \\
\hline & $(0.00234)$ & $(0.00327)$ & $(0.000833)$ & $(0.00343)$ & $(0.00147)$ & $(0.0107)$ \\
\hline \multirow[t]{2}{*}{ Age65_69 } & $0.0107^{\star * \star}$ & -0.000825 & $0.00317^{\star * \star}$ & $0.0431^{* * *}$ & 0.00371 & $-0.158^{\star * *}$ \\
\hline & $(0.00260)$ & $(0.00459)$ & $(0.000929)$ & $(0.00425)$ & $(0.00237)$ & $(0.0139)$ \\
\hline \multirow[t]{2}{*}{ Age70_74 } & $0.0214^{* * *}$ & 0.000734 & $0.00539^{* * *}$ & $0.0852^{* * *}$ & $0.00909^{* * *}$ & $-0.194^{* * *}$ \\
\hline & $(0.00375)$ & $(0.00391)$ & $(0.00143)$ & $(0.00433)$ & $(0.00248)$ & $(0.0150)$ \\
\hline \multirow[t]{2}{*}{ Age75_79 } & $0.0212^{\star \star \star}$ & 0.00332 & $0.00806^{\star \star \star}$ & $0.145^{\star \star \star}$ & $0.0207^{\star \star \star}$ & $-0.213^{* * *}$ \\
\hline & $(0.00451)$ & $(0.00478)$ & $(0.00226)$ & $(0.00761)$ & $(0.00254)$ & $(0.0153)$ \\
\hline \multirow[t]{2}{*}{ AgeAbove_80 } & $0.0210^{* * *}$ & 0.00179 & $0.0143^{* * *}$ & $0.203^{* * *}$ & $0.0405^{* * *}$ & $-0.228^{* * *}$ \\
\hline & $(0.00622)$ & $(0.00519)$ & $(0.00287)$ & $(0.00974)$ & $(0.00336)$ & $(0.0155)$ \\
\hline \multirow[t]{2}{*}{ Married } & $0.00578^{* *}$ & -0.000456 & 0.000519 & $-0.00874^{* *}$ & -0.00174 & 0.00313 \\
\hline & $(0.00285)$ & $(0.00360)$ & $(0.00152)$ & $(0.00386)$ & $(0.00244)$ & $(0.00670)$ \\
\hline \multirow[t]{2}{*}{ Reg_Partnership } & -0.00390 & 0.00234 & 0.00447 & -0.00409 & -0.00155 & 0.00226 \\
\hline & $(0.00629)$ & $(0.00631)$ & $(0.00312)$ & $(0.00835)$ & $(0.00329)$ & $(0.0145)$ \\
\hline \multirow[t]{2}{*}{ Separated } & 0.00731 & 0.00940 & 0.00349 & 0.00269 & -0.000610 & -0.0153 \\
\hline & $(0.00777)$ & $(0.00818)$ & $(0.00329)$ & $(0.00698)$ & $(0.00430)$ & $(0.0193)$ \\
\hline \multirow[t]{2}{*}{ Divorced } & $0.00959^{* * *}$ & $0.0142^{* * \star}$ & -0.000838 & 0.00217 & 0.00218 & $-0.0229^{* * *}$ \\
\hline & $(0.00325)$ & $(0.00404)$ & $(0.00172)$ & $(0.00398)$ & $(0.00290)$ & $(0.00815)$ \\
\hline \multirow[t]{2}{*}{ Widowed } & -0.000305 & -0.000402 & 0.000160 & $0.0116^{\star \star}$ & $0.00622^{\star \star *}$ & $-0.0149^{*}$ \\
\hline & $(0.00325)$ & $(0.00560)$ & $(0.00191)$ & $(0.00566)$ & $(0.00237)$ & $(0.00800)$ \\
\hline \multirow[t]{2}{*}{ Retired } & $-0.0128^{* * *}$ & $-0.0194^{* \star *}$ & -0.00232 & $-0.00842^{*}$ & $-0.0132^{* * *}$ & $0.0914^{\star * \star}$ \\
\hline & $(0.00482)$ & $(0.00746)$ & (0.00199) & $(0.00432)$ & $(0.00292)$ & $(0.0105)$ \\
\hline Employed & $-0.0365^{\star * *}$ & $-0.0323^{* * *}$ & $-0.00404^{* * *}$ & $-0.0211^{* * *}$ & $-0.0148^{* * *}$ & $0.179^{\star * \star}$ \\
\hline & $(0.00369)$ & $(0.00677)$ & $(0.00143)$ & $(0.00242)$ & $(0.00236)$ & $(0.0119)$ \\
\hline Homemaker & $-0.0241^{* * *}$ & $-0.0264^{\star \star \star}$ & $-0.00517^{\star *}$ & $-0.0151^{* \star *}$ & $-0.0111^{* * *}$ & $0.109^{\star * \star}$ \\
\hline & $(0.00472)$ & $(0.00801)$ & $(0.00205)$ & $(0.00440)$ & $(0.00221)$ & $(0.0121)$ \\
\hline Other_Job & $-0.0188^{\star \star}$ & $-0.0296^{\star * \star}$ & -0.00354 & $-0.0184^{* *}$ & $-0.0127^{* * *}$ & $0.104^{* * *}$ \\
\hline & $(0.00880)$ & $(0.00753)$ & $(0.00298)$ & $(0.00837)$ & $(0.00420)$ & $(0.0123)$ \\
\hline N_Children & $-0.00222^{* * *}$ & -0.000611 & 0.000129 & -0.00189 & -0.000394 & $0.00478^{* *}$ \\
\hline & $(0.000758)$ & $(0.000978)$ & $(0.000298)$ & $(0.00128)$ & $(0.000582)$ & $(0.00194)$ \\
\hline N_Grandchildren & 0.000135 & $0.000819^{*}$ & $2.62 \mathrm{e}-05$ & 0.000436 & 0.000108 & $-0.00390^{* * *}$ \\
\hline
\end{tabular}




$\begin{array}{lcccccc} & (0.000316) & (0.000467) & (0.000183) & (0.000453) & (0.000221) & (0.000695) \\ \text { Logincome } & 0.00490^{* * *} & -5.41 \mathrm{e}-05 & 0.000487^{* *} & 0.00254^{*} & 0.000955^{*} & -0.00664^{* * *} \\ & (0.00110) & (0.00110) & (0.000199) & (0.00153) & (0.000565) & (0.00234) \\ \text { Year dummies } & \text { YES } & \text { YES } & \text { YES } & \text { YES } & \text { YES } & \text { YES }\end{array}$

$\begin{array}{llllll}\text { Year dummies } & \text { YES } & \text { YES } & \text { YES } & \text { YES } & \text { YES } \\ \text { Country dummies } & \text { YES } & \text { YES } & \text { YES } & \text { YES } & \text { YES }\end{array}$

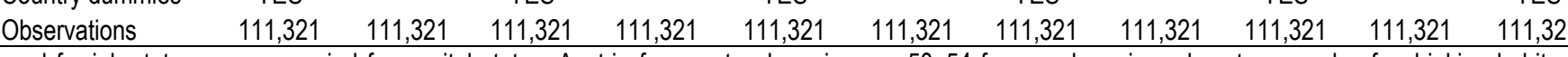
11,321

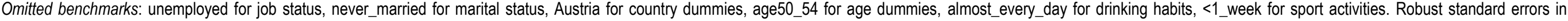
parentheses clustered at country level. ${ }^{* * *} p<0.01,{ }^{* *} p<0.05,{ }^{*} p<0.1$.

Table A5e - Education years and health functionalities (IV estimates)

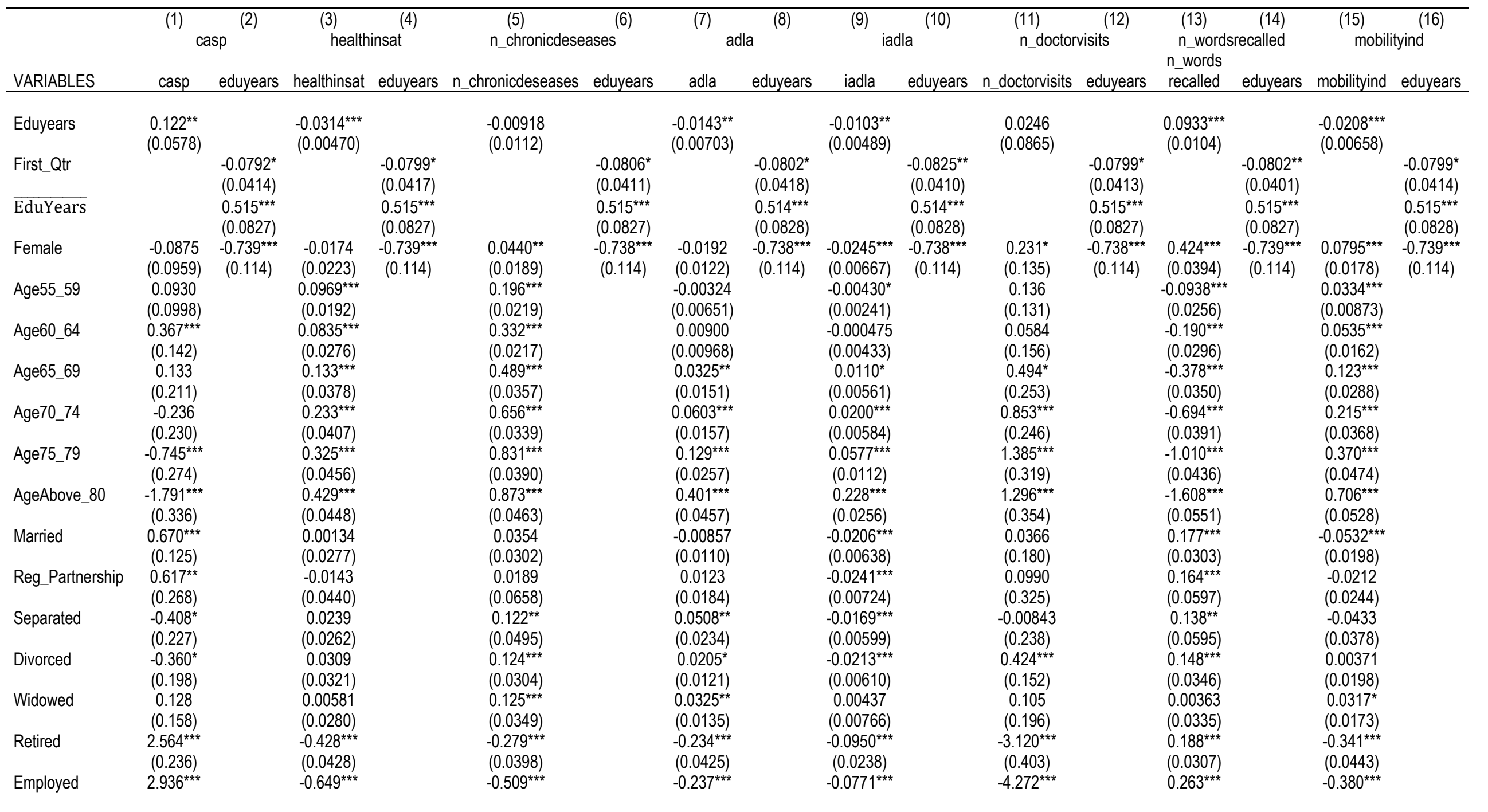




\begin{tabular}{|c|c|c|c|c|c|c|c|c|}
\hline & $(0.257)$ & $(0.0395)$ & $(0.0347)$ & $(0.0319)$ & $(0.0178)$ & $(0.374)$ & $(0.0430)$ & $(0.0335)$ \\
\hline \multirow[t]{2}{*}{ Homemaker } & $2.296^{* \star *}$ & $-0.476^{\star * \star}$ & $-0.361^{* * *}$ & $-0.268^{\star * \star}$ & $-0.0926^{\star \star *}$ & $-3.312^{* \star *}$ & $0.109^{* *}$ & $\begin{array}{l}-0.337^{* * *} \\
(0.0426)\end{array}$ \\
\hline & $(0.231)$ & $(0.0491)$ & $(0.0486)$ & $(0.0426)$ & $(0.0268)$ & $(0.395)$ & $(0.0488)$ & $(0.0426)$ \\
\hline Other_Job & $\begin{array}{c}2.018^{* * *} \\
(0.284)\end{array}$ & $\begin{array}{c}-0.460^{* * *} \\
(0.0457)\end{array}$ & $\begin{array}{c}-0.339^{* * *} \\
(0.0495)\end{array}$ & $\begin{array}{c}-0.161^{* * *} \\
(0.0400)\end{array}$ & $\begin{array}{l}-0.0371 \\
(0.0244)\end{array}$ & $\begin{array}{c}-3.085^{\star * *} \\
(0.430)\end{array}$ & $\begin{array}{c}0.107^{* *} \\
(0.0529)\end{array}$ & $\begin{array}{c}-0.272^{* * *} \\
(0.0409)\end{array}$ \\
\hline N_Children & $\begin{array}{r}-0.0748^{* *} \\
(0.0352)\end{array}$ & $\begin{array}{c}-0.0115^{*} \\
(0.00681)\end{array}$ & $\begin{array}{l}-0.0101^{*} \\
(0.00604)\end{array}$ & $\begin{array}{r}-0.000870 \\
(0.00289)\end{array}$ & $\begin{array}{c}0.00154 \\
(0.00164)\end{array}$ & $\begin{array}{l}-0.00636 \\
(0.0472)\end{array}$ & $\begin{array}{l}-0.0161^{*} \\
(0.00968)\end{array}$ & $\begin{array}{c}0.00694 \\
(0.00429)\end{array}$ \\
\hline N_Grandchildren & $\begin{array}{l}0.0300^{* *} \\
(0.0119)\end{array}$ & $\begin{array}{l}0.00367^{*} \\
(0.00215)\end{array}$ & $\begin{array}{l}0.0143^{* * *} \\
(0.00279)\end{array}$ & $\begin{array}{l}0.00340^{*} \\
(0.00187)\end{array}$ & $\begin{array}{l}0.00226^{\star *} \\
(0.00115)\end{array}$ & $\begin{array}{l}0.0460^{*} \\
(0.0267)\end{array}$ & $\begin{array}{c}-0.00244 \\
(0.00410)\end{array}$ & $\begin{array}{c}0.00579^{* * *} \\
(0.00210)\end{array}$ \\
\hline Logincome & $\begin{array}{l}0.323^{\star \star \star} \\
(0.0481)\end{array}$ & $\begin{array}{c}-0.0242^{\star \star \star} \\
(0.00699)\end{array}$ & $\begin{array}{c}0.00995 \\
(0.00756)\end{array}$ & $\begin{array}{c}0.00730^{\star \star \star} \\
(0.00189)\end{array}$ & $\begin{array}{c}0.00235 \\
(0.00190)\end{array}$ & $\begin{array}{c}0.0730^{*} \\
(0.0404)\end{array}$ & $\begin{array}{c}0.0559^{\star * *} \\
(0.0153)\end{array}$ & $\begin{array}{c}-0.000855 \\
(0.00425)\end{array}$ \\
\hline \multicolumn{9}{|l|}{ DRINKING } \\
\hline 5or6days_week & $\begin{array}{c}0.783^{* * *} \\
(0.139)\end{array}$ & $\begin{array}{l}-0.141^{* * *} \\
(0.0180)\end{array}$ & $\begin{array}{c}-0.0723^{* * *} \\
(0.0249)\end{array}$ & $\begin{array}{c}-0.126^{* * *} \\
(0.0140)\end{array}$ & $\begin{array}{l}-0.0638^{* * *} \\
(0.00843)\end{array}$ & $\begin{array}{c}-1.198^{\star * *} \\
(0.181)\end{array}$ & $\begin{array}{l}0.206^{* * *} \\
(0.0226)\end{array}$ & $\begin{array}{c}-0.168^{* * *} \\
(0.0186)\end{array}$ \\
\hline 3or4days_week & $\begin{array}{c}0.941^{* * *} \\
(0.167)\end{array}$ & $\begin{array}{l}-0.191^{* * *} \\
(0.0151)\end{array}$ & $\begin{array}{c}-0.0871^{* \star *} \\
(0.0285)\end{array}$ & $\begin{array}{c}-0.117^{\star * *} \\
(0.0145)\end{array}$ & $\begin{array}{l}-0.0620^{\star * *} \\
(0.00594)\end{array}$ & $\begin{array}{c}-1.313^{\star \star \star} \\
(0.184)\end{array}$ & $\begin{array}{l}0.273^{\star * \star} \\
(0.0350)\end{array}$ & $\begin{array}{c}-0.196^{\star * *} \\
(0.0154)\end{array}$ \\
\hline 1or2_week & $\begin{array}{c}1.154^{* * *} \\
(0.146)\end{array}$ & $\begin{array}{l}-0.251^{* * *} \\
(0.0181)\end{array}$ & $\begin{array}{l}-0.124^{* * *} \\
(0.0282)\end{array}$ & $\begin{array}{l}-0.127^{* * *} \\
(0.0120)\end{array}$ & $\begin{array}{l}-0.0606^{* * *} \\
(0.00645)\end{array}$ & $\begin{array}{c}-1.627^{* * *} \\
(0.241)\end{array}$ & $\begin{array}{l}0.262^{* * *} \\
(0.0400)\end{array}$ & $\begin{array}{c}-0.204^{* * *} \\
(0.0143)\end{array}$ \\
\hline 1or2_month & $\begin{array}{c}1.066^{* * *} \\
(0.158)\end{array}$ & $\begin{array}{l}-0.282^{* * *} \\
(0.0265)\end{array}$ & $\begin{array}{l}-0.124^{* * *} \\
(0.0312)\end{array}$ & $\begin{array}{c}-0.126^{* * *} \\
(0.0138)\end{array}$ & $\begin{array}{l}-0.0558^{* * *} \\
(0.00742)\end{array}$ & $\begin{array}{c}-1.562^{* * *} \\
(0.290)\end{array}$ & $\begin{array}{l}0.315^{\star * *} \\
(0.0537)\end{array}$ & $\begin{array}{c}-0.196^{* * *} \\
(0.0153)\end{array}$ \\
\hline$<1$ _month & $\begin{array}{c}0.748^{\star * *} \\
(0.121)\end{array}$ & $\begin{array}{l}-0.299^{* * *} \\
(0.0267)\end{array}$ & $\begin{array}{l}-0.143^{* * *} \\
(0.0364)\end{array}$ & $\begin{array}{l}-0.128^{* * *} \\
(0.0148)\end{array}$ & $\begin{array}{l}-0.0572^{* * *} \\
(0.00727)\end{array}$ & $\begin{array}{c}-1.664^{* * *} \\
(0.359)\end{array}$ & $\begin{array}{l}0.382^{* * *} \\
(0.0727)\end{array}$ & $\begin{array}{l}-0.183^{* * *} \\
(0.0219)\end{array}$ \\
\hline 0_in_3months & $\begin{array}{l}1.041^{* *} \\
(0.101)\end{array}$ & $\begin{array}{c}-0.232^{* * *} \\
(0.0189)\end{array}$ & $\begin{array}{c}-0.0831^{* * *} \\
(0.0251)\end{array}$ & $\begin{array}{c}-0.134^{* *} \\
(0.0130)\end{array}$ & $\begin{array}{l}-0.0658^{* * *} \\
(0.00810)\end{array}$ & $\begin{array}{c}-1.893^{* * *} \\
(0.203)\end{array}$ & $\begin{array}{l}0.222^{* \star *} \\
(0.0527)\end{array}$ & $\begin{array}{c}-0.206^{* \star *} \\
(0.0142)\end{array}$ \\
\hline \multicolumn{9}{|l|}{ SPORT } \\
\hline 1_week & $\begin{array}{c}-0.601^{* * *} \\
(0.113)\end{array}$ & $\begin{array}{c}0.107^{\star * *} \\
(0.00967)\end{array}$ & $\begin{array}{l}0.0527^{\star *} \\
(0.0209)\end{array}$ & $\begin{array}{l}-0.00277 \\
(0.00469)\end{array}$ & $\begin{array}{l}-0.00289 \\
(0.00216)\end{array}$ & $\begin{array}{c}0.347^{\star * *} \\
(0.108)\end{array}$ & $\begin{array}{c}-0.00396 \\
(0.0303)\end{array}$ & $\begin{array}{c}0.0351^{* * *} \\
(0.0106)\end{array}$ \\
\hline 1or3_month & $\begin{array}{c}-0.868^{* * *} \\
(0.112)\end{array}$ & $\begin{array}{c}0.165^{\star * *} \\
(0.00987)\end{array}$ & $\begin{array}{l}0.100^{* * *} \\
(0.0204)\end{array}$ & $\begin{array}{l}-0.00852 \\
(0.00736)\end{array}$ & $\begin{array}{l}-0.00409 \\
(0.00371)\end{array}$ & $\begin{array}{c}0.632^{* * *} \\
(0.136)\end{array}$ & $\begin{array}{c}-0.0253 \\
(0.0351)\end{array}$ & $\begin{array}{c}0.0758^{* * *} \\
(0.0180)\end{array}$ \\
\hline Hardlyever_never & $\begin{array}{c}-2.084^{* * *} \\
(0.180)\end{array}$ & $\begin{array}{l}0.505^{\star * *} \\
(0.0185)\end{array}$ & $\begin{array}{l}0.413^{* * *} \\
(0.0389)\end{array}$ & $\begin{array}{l}0.212^{\star * *} \\
(0.0244)\end{array}$ & $\begin{array}{l}0.0712^{\star * *} \\
(0.00941)\end{array}$ & $\begin{array}{c}2.531^{* * *} \\
(0.231)\end{array}$ & $\begin{array}{l}-0.234^{* * *} \\
(0.0341)\end{array}$ & $\begin{array}{l}0.445^{\star * *} \\
(0.0319)\end{array}$ \\
\hline Smoking & $\begin{array}{c}-0.530^{* * *} \\
(0.0844)\end{array}$ & $\begin{array}{c}0.0928^{* * *} \\
(0.0175)\end{array}$ & $\begin{array}{c}-0.0393^{* *} \\
(0.0174)\end{array}$ & $\begin{array}{c}-0.0155^{\star * *} \\
(0.00505)\end{array}$ & $\begin{array}{l}-0.0129^{* * *} \\
(0.00240)\end{array}$ & $\begin{array}{c}-0.602^{* * *} \\
(0.133)\end{array}$ & $\begin{array}{c}-0.0664^{* * *} \\
(0.0209)\end{array}$ & $\begin{array}{l}0.0345^{\star * *} \\
(0.00858)\end{array}$ \\
\hline $\begin{array}{l}\text { Year dummies } \\
\text { Country dummies }\end{array}$ & $\begin{array}{l}\text { YES } \\
\text { YES }\end{array}$ & $\begin{array}{l}\text { YES } \\
\text { YES }\end{array}$ & $\begin{array}{l}\text { YES } \\
\text { YES }\end{array}$ & $\begin{array}{l}\text { YES } \\
\text { YES }\end{array}$ & $\begin{array}{l}\text { YES } \\
\text { YES }\end{array}$ & $\begin{array}{l}\text { YES } \\
\text { YES }\end{array}$ & $\begin{array}{l}\text { YES } \\
\text { YES }\end{array}$ & $\begin{array}{l}\text { YES } \\
\text { YES }\end{array}$ \\
\hline
\end{tabular}

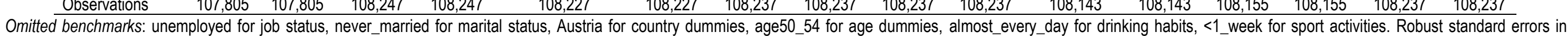
parentheses clustered at country level. ${ }^{* * *} p<0.01,{ }^{* *} p<0.05,{ }^{*} p<0.1$. 
Table A5d - Education years and specific illnesses (IV estimates)

\begin{tabular}{|c|c|c|c|c|c|c|c|c|c|c|c|c|}
\hline \multirow[b]{4}{*}{ VARIABLES } & \multirow{3}{*}{\multicolumn{2}{|c|}{$\begin{array}{l}\text { (1) } \\
\text { longterm } \\
\text { illness }\end{array}$}} & \multirow{3}{*}{\multicolumn{2}{|c|}{$\begin{array}{l}\text { (3) } \\
\text { heartattack }\end{array}$}} & (5) & (6) & (7) & (8) & (9) & (10) & (11) & (12) \\
\hline & & & & & \multirow{2}{*}{\multicolumn{2}{|c|}{ hypertension }} & \multirow{2}{*}{\multicolumn{2}{|c|}{ stroke }} & \multirow{2}{*}{\multicolumn{2}{|c|}{ diabetes }} & \multirow{2}{*}{\multicolumn{2}{|c|}{ asthma }} \\
\hline & & & & & & & & & & & & \\
\hline & 2nd stage & 1st stage & 2nd stage & 1st stage & 2nd stage & 1st stage & 2nd stage & 1st stage & 2nd stage & 1st stage & 2nd stage & 1st stage \\
\hline Eduyears & $\begin{array}{r}-0.000680 \\
(0.00221)\end{array}$ & & $\begin{array}{l}-0.000899 \\
(0.000826)\end{array}$ & & $\begin{array}{c}-0.00461^{* * *} \\
(0.00163)\end{array}$ & & $\begin{array}{l}-0.000676 \\
(0.000692)\end{array}$ & & $\begin{array}{c}-0.000814 \\
(0.00286)\end{array}$ & & $\begin{array}{l}-0.000975 \\
(0.000599)\end{array}$ & \\
\hline First_Qtr & & $\begin{array}{c}-0.0804^{*} \\
(0.0416)\end{array}$ & & $\begin{array}{l}-0.0806^{*} \\
(0.0413)\end{array}$ & & $\begin{array}{l}-0.0793^{*} \\
(0.0414)\end{array}$ & & $\begin{array}{c}-0.0799^{*} \\
(0.0416)\end{array}$ & & $\begin{array}{l}-0.0801^{*} \\
(0.0413)\end{array}$ & & $\begin{array}{c}-0.0805^{*} \\
(0.0417)\end{array}$ \\
\hline$\overline{\text { EduYears }}$ & & $\begin{array}{l}0.515^{\star * *} \\
(0.0826)\end{array}$ & & $\begin{array}{l}0.515^{\star \star *} \\
(0.0827)\end{array}$ & & $\begin{array}{l}0.515^{\star * *} \\
(0.0827)\end{array}$ & & $\begin{array}{l}0.515^{\star \star *} \\
(0.0827)\end{array}$ & & $\begin{array}{l}0.515^{\star * *} \\
(0.0827)\end{array}$ & & $\begin{array}{l}0.515^{\star * *} \\
(0.0827)\end{array}$ \\
\hline Female & $\begin{array}{c}0.00898 \\
(0.00735)\end{array}$ & $\begin{array}{c}-0.739^{\star * *} \\
(0.114)\end{array}$ & $\begin{array}{c}-0.0610^{* * *} \\
(0.00368)\end{array}$ & $\begin{array}{c}-0.738^{* * *} \\
(0.114)\end{array}$ & $\begin{array}{l}0.0207^{* * *} \\
(0.00588)\end{array}$ & $\begin{array}{c}-0.739^{\star * *} \\
(0.114)\end{array}$ & $\begin{array}{c}-0.0169^{* * *} \\
(0.00266)\end{array}$ & $\begin{array}{c}-0.739^{* * *} \\
(0.114)\end{array}$ & $\begin{array}{c}-0.0299^{* * *} \\
(0.00450)\end{array}$ & $\begin{array}{c}-0.739^{* * *} \\
(0.114)\end{array}$ & $\begin{array}{c}0.00162 \\
(0.00146)\end{array}$ & $\begin{array}{c}-0.739^{\star * *} \\
(0.114)\end{array}$ \\
\hline DRINKING & & & & & & & & & & & & \\
\hline 5or6days_week & $\begin{array}{c}-0.0245^{\star * *} \\
(0.00715)\end{array}$ & & $\begin{array}{c}-0.0272^{\star * *} \\
(0.00733)\end{array}$ & & $\begin{array}{c}-0.00234 \\
(0.00732)\end{array}$ & & $\begin{array}{c}-0.0183^{* * *} \\
(0.00478)\end{array}$ & & $\begin{array}{c}-0.0254^{* * *} \\
(0.00301)\end{array}$ & & $\begin{array}{c}-0.00395^{\star *} \\
(0.00164)\end{array}$ & \\
\hline 3or4days_week & $\begin{array}{c}-0.0473^{* * *} \\
(0.00826)\end{array}$ & & $\begin{array}{c}-0.0301^{* * *} \\
(0.00716)\end{array}$ & & $\begin{array}{l}-0.00985 \\
(0.00733)\end{array}$ & & $\begin{array}{c}-0.0198^{* * *} \\
(0.00427)\end{array}$ & & $\begin{array}{c}-0.0361^{* * *} \\
(0.00373)\end{array}$ & & $\begin{array}{c}-0.00453^{* *} \\
(0.00221)\end{array}$ & \\
\hline 1or2_week & $\begin{array}{c}-0.0679^{* * *} \\
(0.00667)\end{array}$ & & $\begin{array}{c}-0.0348^{\star \star *} \\
(0.00617)\end{array}$ & & $\begin{array}{c}-0.0180^{\star \star *} \\
(0.00639)\end{array}$ & & $\begin{array}{c}-0.0216^{\star * *} \\
(0.00457)\end{array}$ & & $\begin{array}{c}-0.0457^{\star \star *} \\
(0.00477)\end{array}$ & & $\begin{array}{c}-0.00435^{\star \star \star} \\
(0.00161)\end{array}$ & \\
\hline 1or2_month & $\begin{array}{c}-0.0588^{* \star *} \\
(0.0102)\end{array}$ & & $\begin{array}{c}-0.0341^{\star * *} \\
(0.00787)\end{array}$ & & $\begin{array}{c}-0.0161^{\star \star *} \\
(0.00540)\end{array}$ & & $\begin{array}{c}-0.0246^{* \star *} \\
(0.00434)\end{array}$ & & $\begin{array}{c}-0.0496^{\star \star *} \\
(0.00559)\end{array}$ & & $\begin{array}{c}-0.00512^{* *} \\
(0.00219)\end{array}$ & \\
\hline$<1$ _month & $\begin{array}{c}-0.0773^{* * *} \\
(0.0113)\end{array}$ & & $\begin{array}{c}-0.0391^{\star * *} \\
(0.00663)\end{array}$ & & $\begin{array}{l}-0.0202^{* *} \\
(0.00793)\end{array}$ & & $\begin{array}{c}-0.0233^{* * *} \\
(0.00369)\end{array}$ & & $\begin{array}{c}-0.0626^{\star * *} \\
(0.00568)\end{array}$ & & $\begin{array}{c}-0.00599^{* *} \\
(0.00247)\end{array}$ & \\
\hline 0_in_3months & $\begin{array}{c}-0.0623^{* * *} \\
(0.00741)\end{array}$ & & $\begin{array}{c}-0.0353^{\star * *} \\
(0.00562)\end{array}$ & & $\begin{array}{c}-0.00214 \\
(0.00684)\end{array}$ & & $\begin{array}{c}-0.0193^{\star * *} \\
(0.00349)\end{array}$ & & $\begin{array}{l}-0.0544^{* * *} \\
(0.00651)\end{array}$ & & $\begin{array}{l}-0.00157^{* *} \\
(0.000767)\end{array}$ & \\
\hline SPORT & & & & & & & & & & & & \\
\hline 1_week & $\begin{array}{c}0.00938 \\
(0.00642)\end{array}$ & & $\begin{array}{c}0.00433 \\
(0.00334)\end{array}$ & & $\begin{array}{l}0.0190^{\star * *} \\
(0.00645)\end{array}$ & & $\begin{array}{l}0.000129 \\
(0.00123)\end{array}$ & & $\begin{array}{c}0.00634^{*} \\
(0.00333)\end{array}$ & & $\begin{array}{l}0.000475 \\
(0.00160)\end{array}$ & \\
\hline 1or3_month & $\begin{array}{l}0.0462^{\star * \star} \\
(0.00790)\end{array}$ & & $\begin{array}{l}0.0200^{* * *} \\
(0.00448)\end{array}$ & & $\begin{array}{l}0.0271^{* * *} \\
(0.00807)\end{array}$ & & $\begin{array}{c}0.00233 \\
(0.00200)\end{array}$ & & $\begin{array}{l}0.0136^{\star * *} \\
(0.00392)\end{array}$ & & $\begin{array}{c}0.00285 \\
(0.00184)\end{array}$ & \\
\hline Hardlyever_never & $\begin{array}{c}0.160^{\star \star *} \\
(0.00988)\end{array}$ & & $\begin{array}{l}0.0675^{\star \star \star} \\
(0.00642)\end{array}$ & & $\begin{array}{l}0.0656^{\star \star \star} \\
(0.00804)\end{array}$ & & $\begin{array}{l}0.0302^{\star * \star} \\
(0.00515)\end{array}$ & & $\begin{array}{l}0.0457^{\star \star *} \\
(0.00450)\end{array}$ & & $\begin{array}{l}0.0122^{\star * \star} \\
(0.00215)\end{array}$ & \\
\hline Smoking & $\begin{array}{l}-0.00927^{*} \\
(0.00545)\end{array}$ & & $\begin{array}{l}-0.0173^{\star * *} \\
(0.00268)\end{array}$ & & $\begin{array}{l}-0.0393^{* * *} \\
(0.00538)\end{array}$ & & $\begin{array}{r}-0.000336 \\
(0.00179)\end{array}$ & & $\begin{array}{l}-0.00712^{* *} \\
(0.00303)\end{array}$ & & $\begin{array}{l}-0.00283 \\
(0.00193)\end{array}$ & \\
\hline Overweight_Obese & $\begin{array}{l}0.0713^{\star * *} \\
(0.00488)\end{array}$ & & $\begin{array}{l}0.0215^{\star \star \star} \\
(0.00404)\end{array}$ & & $\begin{array}{c}0.163^{\star * *} \\
(0.00701)\end{array}$ & & $\begin{array}{l}0.000734 \\
(0.00119)\end{array}$ & & $\begin{array}{l}0.0701^{* * *} \\
(0.00344)\end{array}$ & & $\begin{array}{c}0.00476^{\star * *} \\
(0.00167)\end{array}$ & \\
\hline Age55_59 & $\begin{array}{l}0.0397^{\star * \star} \\
(0.00865)\end{array}$ & & $\begin{array}{c}0.00721^{* \star *} \\
(0.00214)\end{array}$ & & $\begin{array}{l}0.0623^{\star \star \star} \\
(0.00757)\end{array}$ & & $\begin{array}{c}0.00265^{\star} \\
(0.00140)\end{array}$ & & $\begin{array}{l}0.0151^{\star * *} \\
(0.00335)\end{array}$ & & $\begin{array}{l}-0.00437^{\star} \\
(0.00230)\end{array}$ & \\
\hline Age60_64 & $\begin{array}{l}0.0401^{\star * *} \\
(0.00905)\end{array}$ & & $\begin{array}{l}0.0199^{\star \star \star} \\
(0.00384)\end{array}$ & & $\begin{array}{c}0.112^{\star \star \star} \\
(0.00953)\end{array}$ & & $\begin{array}{l}0.00372^{\star *} \\
(0.00156)\end{array}$ & & $\begin{array}{l}0.0254^{\star * *} \\
(0.00402)\end{array}$ & & $\begin{array}{l}-0.00503^{*} \\
(0.00286)\end{array}$ & \\
\hline Age65_69 & $\begin{array}{c}0.0597^{\star * *} \\
(0.0113)\end{array}$ & & $\begin{array}{l}0.0396^{* * *} \\
(0.00346)\end{array}$ & & $\begin{array}{l}0.156^{\star \star \star} \\
(0.0137)\end{array}$ & & $\begin{array}{l}0.0103^{* * *} \\
(0.00240)\end{array}$ & & $\begin{array}{l}0.0471^{* * *} \\
(0.00745)\end{array}$ & & $\begin{array}{c}-0.00754^{* *} \\
(0.00351)\end{array}$ & \\
\hline Age70_74 & $0.0848^{* * *}$ & & $0.0773^{* * *}$ & & $0.191^{* \star *}$ & & $0.0173^{* * *}$ & & $0.0520^{* * *}$ & & -0.00404 & \\
\hline
\end{tabular}




\begin{tabular}{|c|c|c|c|c|c|c|c|c|}
\hline & $(0.0129)$ & $(0.00454)$ & $(0.0120)$ & $(0.00332)$ & & $(0.00547)$ & $(0.00382)$ & \\
\hline \multirow[t]{2}{*}{ Age75_79 } & $0.107^{* * *}$ & $0.120^{* * *}$ & $0.199^{\star * *}$ & $0.0355^{\star * *}$ & & $0.0553^{\star * *}$ & -0.00532 & \\
\hline & $(0.0169)$ & $(0.00647)$ & $(0.0119)$ & $(0.00441)$ & & $(0.00827)$ & $(0.00361)$ & \\
\hline \multirow[t]{2}{*}{ AgeAbove_80 } & $0.124^{\star \star \star}$ & $0.143^{\star * *}$ & $0.173^{* \star *}$ & $0.0459^{* \star *}$ & & $0.0415^{\star \star \star}$ & $-0.00822^{*}$ & \\
\hline & $(0.0168)$ & $(0.0106)$ & $(0.0147)$ & $(0.00525)$ & & $(0.00795)$ & $(0.00433)$ & \\
\hline \multirow[t]{2}{*}{ Married } & -0.00691 & $0.0123^{*}$ & 0.0140 & 0.00400 & & -0.00819 & -0.00204 & \\
\hline & $(0.00785)$ & $(0.00739)$ & (0.00958) & $(0.00318)$ & & $(0.00597)$ & $(0.00314)$ & \\
\hline \multirow[t]{2}{*}{ Reg_Partnership } & -0.00832 & 0.00813 & -0.0133 & 0.00224 & & -0.00805 & 0.00501 & \\
\hline & $(0.0176)$ & $(0.0164)$ & $(0.0159)$ & $(0.00478)$ & & $(0.0111)$ & $(0.00749)$ & \\
\hline \multirow[t]{2}{*}{ Separated } & 0.00810 & 0.00844 & 0.00942 & $0.0240^{\star * \star}$ & & 0.0175 & -0.00252 & \\
\hline & $(0.0189)$ & $(0.0133)$ & $(0.0154)$ & $(0.00740)$ & & $(0.0131)$ & $(0.00437)$ & \\
\hline \multirow[t]{2}{*}{ Divorced } & $0.0332^{* * *}$ & $0.0238^{* * *}$ & -0.00209 & $0.00645^{*}$ & & -0.00412 & $0.00864^{* * *}$ & \\
\hline & $(0.0113)$ & $(0.00903)$ & $(0.00807)$ & $(0.00390)$ & & $(0.00653)$ & $(0.00296)$ & \\
\hline \multirow[t]{2}{*}{ Widowed } & 0.00276 & $0.0171^{* * *}$ & $0.0458^{\star * *}$ & 0.00230 & & 0.00524 & 0.000720 & \\
\hline & $(0.00973)$ & $(0.00639)$ & $(0.0112)$ & $(0.00371)$ & & $(0.00709)$ & $(0.00354)$ & \\
\hline \multirow[t]{2}{*}{ Retired } & $-0.180^{\star \star \star}$ & $-0.0368^{\star * *}$ & $-0.0265^{\star \star \star}$ & $-0.0286^{\star \star *}$ & & $-0.0289^{\star * \star}$ & $-0.00927^{\star *}$ & \\
\hline & $(0.0197)$ & (0.00758) & $(0.00896)$ & $(0.00492)$ & & $(0.00697)$ & (0.00438) & \\
\hline \multirow[t]{2}{*}{ Employed } & $-0.277^{\star * *}$ & $-0.0678^{* * *}$ & $-0.0552^{* * *}$ & $-0.0425^{\star * *}$ & & $-0.0489^{* * *}$ & $-0.0160^{* * *}$ & \\
\hline & $(0.0177)$ & $(0.00719)$ & $(0.0112)$ & $(0.00448)$ & & $(0.00668)$ & $(0.00468)$ & \\
\hline \multirow[t]{2}{*}{ Homemaker } & $-0.219^{* * *}$ & $-0.0540^{\star * \star}$ & $-0.0268^{* *}$ & $-0.0359^{\star * *}$ & & $-0.0288^{* * *}$ & $-0.0116^{\star \star *}$ & \\
\hline & $(0.0203)$ & $(0.00843)$ & (0.0111) & $(0.00626)$ & & $(0.00849)$ & $(0.00426)$ & \\
\hline \multirow[t]{2}{*}{ Other_Job } & $-0.178^{\star \star \star}$ & $-0.0380^{\star * *}$ & $-0.0405^{\star *}$ & $-0.0258^{\star * *}$ & & $-0.0343^{* *}$ & $-0.0226^{* * *}$ & \\
\hline & $(0.0302)$ & $(0.0108)$ & $(0.0182)$ & $(0.00712)$ & & $(0.0159)$ & $(0.00741)$ & \\
\hline \multirow[t]{2}{*}{ N_Children } & -0.00269 & 0.000287 & -0.00154 & 0.00122 & & 0.00191 & $0.000854^{*}$ & \\
\hline & $(0.00220)$ & $(0.00137)$ & $(0.00135)$ & (0.000966) & & (0.00139) & $(0.000513)$ & \\
\hline \multirow[t]{2}{*}{ N_Grandchildren } & $0.00405^{* * *}$ & $0.00268^{* * *}$ & $0.00213^{\star * *}$ & 0.000113 & & $0.00147^{\star *}$ & $0.000626^{* * *}$ & \\
\hline & $(0.000614)$ & $(0.000641)$ & $(0.000660)$ & $(0.000448)$ & & $(0.000619)$ & $(0.000242)$ & \\
\hline \multirow[t]{2}{*}{ Logincome } & $0.00561^{* *}$ & 0.00117 & -0.00122 & $0.00147^{\star *}$ & & -0.000863 & $0.00219^{\star \star}$ & \\
\hline & $(0.00270)$ & $(0.00132)$ & $(0.00185)$ & $(0.000630)$ & & $(0.00120)$ & $(0.00107)$ & \\
\hline Year dummies & YES & YES & YES & YES & & YES & YES & \\
\hline Country dummies & YES & YES & YES & YES & & YES & YES & \\
\hline Observations & 108,251 & 108,251 & 108,227 & 108,227 & 108,227 & 108,227 & 108,227 & 108,227 \\
\hline
\end{tabular}

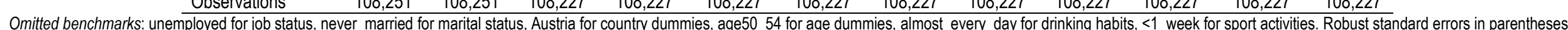
clustered at country level. ${ }^{* * *} p<0.01,{ }^{* *} p<0.05,{ }^{*} p<0.1$.

Table A5d - Education years and specific illnesses (IV estimates) - continued

\begin{tabular}{|c|c|c|c|c|c|c|c|c|c|c|c|c|c|c|}
\hline \multirow[b]{3}{*}{ VARIABLES } & (13) & (14) & (15) & $(16)$ & (17) & (18) & (19) & (20) & (21) & (22) & (23) & (24) & (25) & (26) \\
\hline & \multicolumn{2}{|c|}{ arthritis } & \multicolumn{2}{|c|}{ cancer } & \multicolumn{2}{|c|}{ ulcer } & \multicolumn{2}{|c|}{ parkinson } & \multicolumn{2}{|c|}{ cataracts } & \multicolumn{2}{|c|}{ fracture } & \multicolumn{2}{|c|}{ none } \\
\hline & 2nd st. & $1 \mathrm{st}$ st. & 2nd st. & 1st st. & 2nd st. & 1st st. & 2nd st. & $1 \mathrm{st}$ st. & 2nd st. & 1st st. & 2nd st. & $1 \mathrm{st}$ st. & 2nd st. & 1st st. \\
\hline Eduyears & $\begin{array}{l}-0.00206 \\
(0.00219)\end{array}$ & & $\begin{array}{l}0.00264^{* * *} \\
(0.000918)\end{array}$ & & $\begin{array}{l}-0.000746 \\
(0.000849)\end{array}$ & & $\begin{array}{c}-0.000616 \\
(0.000439)\end{array}$ & & $\begin{array}{l}0.00298^{*} \\
(0.00170)\end{array}$ & & $\begin{array}{l}-0.000331 \\
(0.000520)\end{array}$ & & $\begin{array}{l}0.000244 \\
(0.00185)\end{array}$ & \\
\hline
\end{tabular}




\begin{tabular}{|c|c|c|c|c|c|c|c|c|c|c|c|c|c|c|}
\hline First_Qtr & & $\begin{array}{l}-0.0800^{*} \\
(0.0416)\end{array}$ & & $\begin{array}{l}-0.0814^{*} \\
(0.0417)\end{array}$ & & $\begin{array}{l}-0.0800^{*} \\
(0.0414)\end{array}$ & & $\begin{array}{l}-0.0814^{*} \\
(0.0416)\end{array}$ & & $\begin{array}{l}-0.0786^{*} \\
(0.0413)\end{array}$ & & $\begin{array}{l}-0.0799^{*} \\
(0.0414)\end{array}$ & & $\begin{array}{l}-0.0800^{*} \\
(0.0414)\end{array}$ \\
\hline$\overline{\text { EduYears }}$ & & $\begin{array}{l}0.515^{\star * *} \\
(0.0826)\end{array}$ & & $\begin{array}{l}0.515^{\star * *} \\
(0.0826)\end{array}$ & & $\begin{array}{l}0.515^{\star * *} \\
(0.0827)\end{array}$ & & $\begin{array}{l}0.515^{\star * *} \\
(0.0827)\end{array}$ & & $\begin{array}{l}0.515^{\star * *} \\
(0.0827)\end{array}$ & & $\begin{array}{l}0.515^{\star * *} \\
(0.0827)\end{array}$ & & $\begin{array}{l}0.515^{* * *} \\
(0.0826)\end{array}$ \\
\hline Female & $\begin{array}{l}0.106^{* * *} \\
(0.0107)\end{array}$ & $\begin{array}{c}-0.739^{\star \star *} \\
(0.114)\end{array}$ & $\begin{array}{l}0.00607^{* *} \\
(0.00246)\end{array}$ & $\begin{array}{c}-0.739^{\star \star *} \\
(0.114)\end{array}$ & $\begin{array}{c}-0.0101^{* * *} \\
(0.00278)\end{array}$ & $\begin{array}{c}-0.739^{* * *} \\
(0.114)\end{array}$ & $\begin{array}{c}-0.00363^{\star * *} \\
(0.000696)\end{array}$ & $\begin{array}{c}-0.739^{* * *} \\
(0.114)\end{array}$ & $\begin{array}{l}0.0232^{\star * *} \\
(0.00222)\end{array}$ & $\begin{array}{c}-0.739^{* \star *} \\
(0.114)\end{array}$ & $\begin{array}{c}0.00237^{*} \\
(0.00132)\end{array}$ & $\begin{array}{c}-0.739^{\star * *} \\
(0.114)\end{array}$ & $\begin{array}{c}-0.0179^{\star * *} \\
(0.00455)\end{array}$ & $\begin{array}{c}-0.739^{* * *} \\
(0.114)\end{array}$ \\
\hline DRINKING & & & & & & & & & & & & & & \\
\hline 5or6days_week & $\begin{array}{l}0.000468 \\
(0.00605)\end{array}$ & & $\begin{array}{c}-0.00638^{* * *} \\
(0.00202)\end{array}$ & & $\begin{array}{c}-0.00352 \\
(0.00412)\end{array}$ & & $\begin{array}{c}-0.00361^{* * *} \\
(0.000909)\end{array}$ & & $\begin{array}{c}0.00436 \\
(0.00445)\end{array}$ & & $\begin{array}{c}-0.00118 \\
(0.00173)\end{array}$ & & $\begin{array}{l}-0.00481 \\
(0.00717)\end{array}$ & \\
\hline 3or4days_week & $\begin{array}{c}0.00547 \\
(0.00582)\end{array}$ & & $\begin{array}{c}-0.00482^{* *} \\
(0.00214)\end{array}$ & & $\begin{array}{l}-0.00320 \\
(0.00362)\end{array}$ & & $\begin{array}{c}-0.00376^{* * *} \\
(0.00106)\end{array}$ & & $\begin{array}{c}0.00475 \\
(0.00383)\end{array}$ & & $\begin{array}{l}-0.00212 \\
(0.00138)\end{array}$ & & $\begin{array}{c}0.00670 \\
(0.00629)\end{array}$ & \\
\hline 1or2_week & $\begin{array}{c}0.00157 \\
(0.00624)\end{array}$ & & $\begin{array}{c}-0.00700^{* * *} \\
(0.00227)\end{array}$ & & $\begin{array}{l}-0.00591 \\
(0.00454)\end{array}$ & & $\begin{array}{c}-0.00466^{* * *} \\
(0.00105)\end{array}$ & & $\begin{array}{c}0.00307 \\
(0.00409)\end{array}$ & & $\begin{array}{l}-0.00123 \\
(0.00156)\end{array}$ & & $\begin{array}{c}0.0119^{*} \\
(0.00632)\end{array}$ & \\
\hline 1or2_month & $\begin{array}{c}0.00158 \\
(0.00794)\end{array}$ & & $\begin{array}{l}-0.00726^{*} \\
(0.00393)\end{array}$ & & $\begin{array}{c}-0.00784^{* *} \\
(0.00346)\end{array}$ & & $\begin{array}{c}-0.00472^{* * *} \\
(0.00114)\end{array}$ & & $\begin{array}{c}0.00312 \\
(0.00426)\end{array}$ & & $\begin{array}{c}-0.000184 \\
(0.00185)\end{array}$ & & $\begin{array}{l}0.0174^{*} \\
(0.0102)\end{array}$ & \\
\hline$<1$ month & $\begin{array}{l}-0.00259 \\
(0.00832)\end{array}$ & & $\begin{array}{l}-0.00318 \\
(0.00494)\end{array}$ & & $\begin{array}{l}-0.00564 \\
(0.00602)\end{array}$ & & $\begin{array}{c}-0.00543^{* * *} \\
(0.00151)\end{array}$ & & $\begin{array}{c}0.00450 \\
(0.00661)\end{array}$ & & $\begin{array}{l}-0.000760 \\
(0.00270)\end{array}$ & & $\begin{array}{c}0.0209 \\
(0.0129)\end{array}$ & \\
\hline 0_in_3months & $\begin{array}{c}0.00531 \\
(0.00726)\end{array}$ & & $\begin{array}{c}-0.00566^{* *} \\
(0.00253)\end{array}$ & & $\begin{array}{c}-0.00684^{* *} \\
(0.00336)\end{array}$ & & $\begin{array}{c}-0.00524^{\star * *} \\
(0.000946)\end{array}$ & & $\begin{array}{c}0.00841^{* * *} \\
(0.00295)\end{array}$ & & $\begin{array}{r}-0.000516 \\
(0.00162)\end{array}$ & & $\begin{array}{c}0.00416 \\
(0.00802)\end{array}$ & \\
\hline SPORT & & & & & & & & & & & & & & \\
\hline 1_week & $\begin{array}{c}0.00525 \\
(0.00409)\end{array}$ & & $\begin{array}{c}0.00170 \\
(0.00253)\end{array}$ & & $\begin{array}{l}-0.000247 \\
(0.00274)\end{array}$ & & $\begin{array}{c}-0.000216 \\
(0.000465)\end{array}$ & & $\begin{array}{c}0.00353 \\
(0.00319)\end{array}$ & & $\begin{array}{c}-8.25 \mathrm{e}-06 \\
(0.000981)\end{array}$ & & $\begin{array}{l}-0.0176^{* *} \\
(0.00716)\end{array}$ & \\
\hline 1or3_month & $\begin{array}{c}0.00751 \\
(0.00564)\end{array}$ & & $\begin{array}{c}0.00210 \\
(0.00279)\end{array}$ & & $\begin{array}{l}0.000263 \\
(0.00220)\end{array}$ & & $\begin{array}{c}-6.06 e-05 \\
(0.000575)\end{array}$ & & $\begin{array}{l}0.000589 \\
(0.00237)\end{array}$ & & $\begin{array}{l}0.000938 \\
(0.00159)\end{array}$ & & $\begin{array}{c}-0.0426^{* * *} \\
(0.00686)\end{array}$ & \\
\hline Hardlyever_never & $\begin{array}{l}0.0622^{* * *} \\
(0.00714)\end{array}$ & & $\begin{array}{l}0.0226^{* * *} \\
(0.00187)\end{array}$ & & $\begin{array}{l}0.0128^{* * *} \\
(0.00287)\end{array}$ & & $\begin{array}{l}0.00631^{* * *} \\
(0.000703)\end{array}$ & & $\begin{array}{l}0.0198^{\star * \star} \\
(0.00320)\end{array}$ & & $\begin{array}{l}0.0108^{\star * *} \\
(0.00135)\end{array}$ & & $\begin{array}{c}-0.0934^{* * *} \\
(0.00709)\end{array}$ & \\
\hline Smoking & $\begin{array}{l}-0.00305 \\
(0.00407)\end{array}$ & & $\begin{array}{l}-0.00436^{*} \\
(0.00226)\end{array}$ & & $\begin{array}{l}0.0199^{\star * \star} \\
(0.00303)\end{array}$ & & $\begin{array}{c}-0.00207^{\star * *} \\
(0.000587)\end{array}$ & & $\begin{array}{l}-0.000815 \\
(0.00232)\end{array}$ & & $\begin{array}{l}0.000251 \\
(0.00110)\end{array}$ & & $\begin{array}{c}0.00334 \\
(0.00334)\end{array}$ & \\
\hline Overweight_Obese & $\begin{array}{l}0.0474^{* * *} \\
(0.00487)\end{array}$ & & $\begin{array}{c}-0.00435^{\star * *} \\
(0.00145)\end{array}$ & & $\begin{array}{c}-0.000754 \\
(0.00256)\end{array}$ & & $\begin{array}{l}-0.00150^{* * *} \\
(0.000557)\end{array}$ & & $\begin{array}{l}0.00470^{* *} \\
(0.00208)\end{array}$ & & $\begin{array}{l}-0.000985 \\
(0.000779)\end{array}$ & & $\begin{array}{c}-0.0870^{* * *} \\
(0.00279)\end{array}$ & \\
\hline Age55_59 & $\begin{array}{l}0.0462^{* * *} \\
(0.00655)\end{array}$ & & $\begin{array}{l}0.00450^{*} \\
(0.00267)\end{array}$ & & $\begin{array}{c}-0.00149 \\
(0.00400)\end{array}$ & & $\begin{array}{c}-3.13 e-05 \\
(0.000466)\end{array}$ & & $\begin{array}{c}0.00524^{* * *} \\
(0.00171)\end{array}$ & & $\begin{array}{l}0.000145 \\
(0.00135)\end{array}$ & & $\begin{array}{c}-0.0835^{\star \star \star} \\
(0.0109)\end{array}$ & \\
\hline Age60_64 & $\begin{array}{l}0.0670^{* * *} \\
(0.00910)\end{array}$ & & $\begin{array}{c}0.00865^{\star \star *} \\
(0.00235)\end{array}$ & & $\begin{array}{c}-0.000488 \\
(0.00327)\end{array}$ & & $\begin{array}{c}0.00152^{*} \\
(0.000826)\end{array}$ & & $\begin{array}{l}0.0194^{* * *} \\
(0.00343)\end{array}$ & & $\begin{array}{c}0.00182 \\
(0.00146)\end{array}$ & & $\begin{array}{c}-0.125^{\star * *} \\
(0.0107)\end{array}$ & \\
\hline Age65_69 & $\begin{array}{c}0.0877^{\star * \star} \\
(0.0137)\end{array}$ & & $\begin{array}{l}0.0107^{\star * \star} \\
(0.00261)\end{array}$ & & $\begin{array}{r}-0.000824 \\
(0.00459)\end{array}$ & & $\begin{array}{l}0.00318^{* \star *} \\
(0.000924)\end{array}$ & & $\begin{array}{l}0.0431^{\star \star \star} \\
(0.00425)\end{array}$ & & $\begin{array}{c}0.00371 \\
(0.00237)\end{array}$ & & $\begin{array}{c}-0.158^{* \star \star} \\
(0.0139)\end{array}$ & \\
\hline Age70_74 & $\begin{array}{l}0.100^{\star * \star} \\
(0.0161)\end{array}$ & & $\begin{array}{l}0.0214^{* * *} \\
(0.00375)\end{array}$ & & $\begin{array}{l}0.000735 \\
(0.00390)\end{array}$ & & $\begin{array}{c}0.00539^{* * *} \\
(0.00143)\end{array}$ & & $\begin{array}{l}0.0852^{* * *} \\
(0.00433)\end{array}$ & & $\begin{array}{c}0.00909^{* * *} \\
(0.00247)\end{array}$ & & $\begin{array}{c}-0.194^{* \star *} \\
(0.0150)\end{array}$ & \\
\hline Age75_79 & $\begin{array}{l}0.123^{* \star *} \\
(0.0169)\end{array}$ & & $\begin{array}{l}0.0212^{\star * *} \\
(0.00452)\end{array}$ & & $\begin{array}{c}0.00332 \\
(0.00477)\end{array}$ & & $\begin{array}{c}0.00806^{* * *} \\
(0.00225)\end{array}$ & & $\begin{array}{c}0.145^{\star \star *} \\
(0.00761)\end{array}$ & & $\begin{array}{l}0.0207^{\star \star \star} \\
(0.00253)\end{array}$ & & $\begin{array}{c}-0.213^{\star \star *} \\
(0.0153)\end{array}$ & \\
\hline AgeAbove_80 & $\begin{array}{l}0.146^{* * *} \\
(0.0183)\end{array}$ & & $\begin{array}{l}0.0210^{* * *} \\
(0.00623)\end{array}$ & & $\begin{array}{c}0.00179 \\
(0.00518)\end{array}$ & & $\begin{array}{l}0.0143^{* * *} \\
(0.00288)\end{array}$ & & $\begin{array}{c}0.203^{* * *} \\
(0.00973)\end{array}$ & & $\begin{array}{l}0.0405^{\star * *} \\
(0.00336)\end{array}$ & & $\begin{array}{c}-0.228^{* * *} \\
(0.0155)\end{array}$ & \\
\hline Married & $\begin{array}{l}-0.00289 \\
(0.00868)\end{array}$ & & $\begin{array}{l}0.00579 * * \\
(0.00285)\end{array}$ & & $\begin{array}{r}-0.000453 \\
(0.00360)\end{array}$ & & $\begin{array}{l}0.000514 \\
(0.00152)\end{array}$ & & $\begin{array}{c}-0.00873^{* *} \\
(0.00386)\end{array}$ & & $\begin{array}{c}-0.00173 \\
(0.00244)\end{array}$ & & $\begin{array}{c}0.00312 \\
(0.00670)\end{array}$ & \\
\hline Reg_Partnership & $\begin{array}{r}-0.00247 \\
(0.0134)\end{array}$ & & $\begin{array}{l}-0.00390 \\
(0.00629)\end{array}$ & & $\begin{array}{c}0.00233 \\
(0.00632)\end{array}$ & & $\begin{array}{c}0.00447 \\
(0.00312)\end{array}$ & & $\begin{array}{l}-0.00408 \\
(0.00836)\end{array}$ & & $\begin{array}{c}-0.00155 \\
(0.00330)\end{array}$ & & $\begin{array}{l}0.00225 \\
(0.0145)\end{array}$ & \\
\hline
\end{tabular}




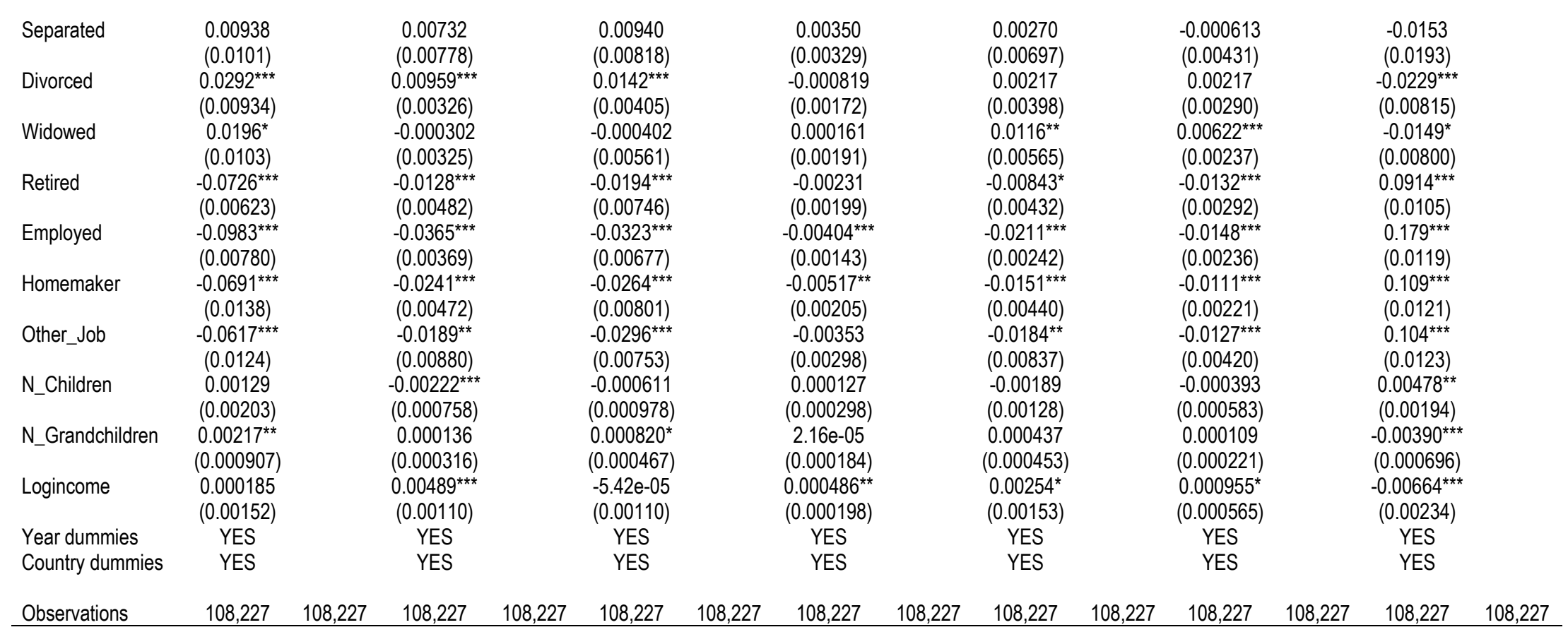

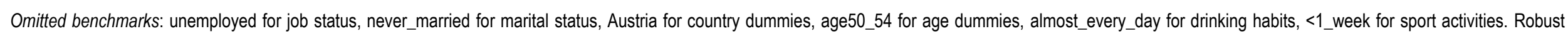
standard errors in parentheses clustered at country level. ${ }^{* * *} p<0.01,{ }^{* *} p<0.05,{ }^{*} p<0.1$. 